
Printed at the Mathematical Centre, 413 Kruislaan, Amsterdam.

The Mathematical Centre, founded the 11-th of February 1946, is a nonprofit institution aiming at the promotion of pure mathematics and its applications. It is sponsored by the Netherlands Government through the Netherlands Organization for the Advancement of Pure Research (Z.W.0.). 
MATHEMATICAL CENTRE TRACTS 128

\section{VARIABLE DIMENSION FIXED POINT ALGORITHMS AND TRIANGULATIONS}

A.J.J. TALMAN

WITH THE COLLABORATION OF G. VAN DER LAAN 
ACKNOWLEDGEMENTS

I am most grateful to professor H.C. Tijms, and professor A.H.Q.M. Merkies for arousing my interest in the field of fixed point algorithms and their constant encouragement and to professor R. Saigal and professor M.J. Todd for the stimulating discussions and correspondence.

Most part of the monograph evolved from joint research with

Gerard van der Laan to whom I am indebted for the inspiring and helpful

discussions we had.

Finally, I want to thank Henk Kruijer and Gloria Wagenaar for their excellent typing and Arie Oudshoorn for his help in running the computer programs.

I thank the Mathematical Centre for the opportunity to publish this monograph in their series Mathematical Centre Tracts and all those at the Mathematical Centre who have contributes to its technical realization. 



\section{CONTENTS}

CHAPTER 1. INTRODUCTION AND SUMMARY.

CHAPTER 2. PRELIMINARIES.

2.1. Introduction. 6

2.2. Triangulations and subdivisions. 6

2.3. Brouwer's theorem and Sperner's lemma. 11

2.4. Kakutani's theorem. 13

2.5. Special triangulations of $R^{n}$. 16

2.6. Triangulations of $\mathrm{S}^{\mathrm{n}}$. 20

CHAPTER 3. EXTENSIONS AND APPLICATIONS OF FIXED POINT THEOREMS. 26

3.1. Introduction. 26

3.2. Noncooperative N-person game. 26

3.3. Extensions to Kakutani's theorem. 28

3.4. Constrained optimization. 30

3.5. Nonlinear complementarity problem. 32

CHAPTER 4. ALGORITHMS TO FIND COMPLETELY LABELLED SIMPLICES. 37

4.1. Introduction. 37

4.2. Labelling and accuracy. 38

4.3. Kuhn's and Eaves' first algorithms. 44

4.4. Merrill's algorithm. 49

4.5. Van der Laan and Talman's basic algorithm. 52

CHAPTER 5. THE EFFICIENCY OF TRIANGULATIONS. 59

5.1. Introduction. $\quad 59$

5.2. Fixed point triangulations of $R^{n}$. 60

5.3. Crude measures. 63

5.4. Average directional density. 64

5.5. Measures based on minimizing the number of grid points. 69

5.6. Comparison of several triangulations of $R^{n}$. 76

5.7. A good triangulation of the affine hull of $\mathrm{s}^{\mathrm{n}}$. 80 
CHAPTER 6. VARIABLE DIMENSION FIXED POINT ALGORITHM ON THE PRODUCT SPACE S OF UNIT SIMPLICES.

6.1. Introduction. 85

6.2. Triangulation of S. 87

6.3. Integer labelling and approximation. 96

6.4. The variable dimension algorithm on $\mathrm{S}$. 99

6.5. Vector labelling. 105

6.6. Computational experience. 112

CHAPTER 7. CLASS OF VARIABLE DIMENSION FIXED POINT ALGORITHMS ON $R^{\mathrm{n}}$. 116

7.1. Introduction.

7.2. Triangulation of $R^{\mathrm{n}}$ for given positive integers summing up to $\mathrm{n}$.

7.3. Integer labelling and approximation.

120

7.4. The variable dimension algorithm on $R^{n}$.

123

7.5. Vector labelling.

7.6. The two extreme cases $N=1$ and $N=n$.

7.7. Computational experience.

CHAPTER 8. GEOMETRIC INTERPRETATIONS OF THE ALGORITHMS.

8.1. Introduction. 150

8.2. Interpretation with the set $S$ on the zero-level. 151

8.3. Two interpretations with $M+N$ points. 155

8.4. Modification for the algorithm on $R^{n}$. 159

REFERENCES. 
CHAPTER 1

INTRODUCTION AND SUMMARY

Fixed point theorems are widely used in existence proofs of solutions to nonlinear problems. Numerous applications include the existence of an equilibrium strategy vector in noncooperative $\mathrm{N}$-person games, the existence of a price equilibrium in economic models, and the existence of a solution to (un) constrained optimization problems and nonlinear complementarity problems. The most famous fixed point theorems are those of Brouwer [1912] and Kakutani [1941]. Brouwer's theorem is concerned with the existence of a fixed point of a continuous funcion from a compact, convex set into itself. Kakutani generalized Brouwer's theorem to upper semi-continuous point to set mappings. However, the original proofs were not constructive. It is only recently that algorithms heve been developed to compute (approximate) fixed points. The pioneering work on fixed point algorithms was done by Scarf [1967] (see also Scarf [1973]), who introduced a fixed point algorithm on the unit simplex $s^{n}$. His algorithm generates a path of adjacent so-called primitive sets. This path starts in a corner of $\mathrm{s}^{\mathrm{n}}$ and terminates as soon as a completely labelled primitive set is found yielding an approximate fixed point. An essential part of Scarf's algorithm is the unique replacement step by which it can be proved that the algorithm always terminates within a finite number of steps. This proof is based on the work of Lemke [1965] (see also Lemke and Howson [1964]), who developed an algorithm to compute an equilibrium strategy for a two-person non-zero-sum game.

In 1968 Hansen improved Scarf's algorithm by developing an efficient and simple scheme for the replacement step. About the same time Kuhn $[1968,1969]$ developed two algorithms on $S^{\mathrm{n}}$ using the same steps. Kuhn pointed out that these steps describe the replacement step between two adjacent simplices of a (regular) triangulation introduced already by Freudenthal [1942]. Almost all recent fixed point algorithms are based on 
the concept of a triangulation of a set.

The algorithms mentioned above suffer from the computational disadvantage that the grid of the triangulation is fixed throughout the algorithm while a start must be made outside the region of interest (see also Eaves [1971]). A first more sophisticated method is due to Merrill [1971, 1972], later independently found by Kuhn and Mackinnon [1975], Lüthi [1975, 1976] and Fisher, Gould and Tolle [1977]. This algorithm known as the Sandwichmethod can start anywhere but requires the addition of an extra dimension. Thus, as soon as for some grid size an approximate fixed point is found, this point can be used to provide the new starting point in a new application of the algorithm with a finer grid. By this way the accuracy can be rapidly improved.

A second method was discovered independently and simultaneously by Eaves [1972] on $S^{n}$ and by Eaves and Saigal [1972] on $R^{n}$. In their algorithm the mesh of the triangulation is automatically refined. Both algorithms have the computational disadvantage that they are always operating with $(n+1)$-dimensional simplices. Moreover, the Sandwich-method needs artificial points to avoid cycling whereas the continuous-deformation method can only be applied for incrementation factors of at most two. Algorithms without an extra dimension were developed by Tuy [1979] (see also Van der Heijden [1979]) and Garcia and Gould [1976, 1979]. However, as argued by Todd [1978b], Tuy's method needs a lot of computation time whereas Garcia and Gould's method may fail. Another restart algorithm without an extra dimension was developed by Van der Laan and Talman [1979a, 1979b]. Their algorithm has the advantage that it starts with a single point and generates a path of adjacent simplices of variable dimension until a completely labelled simplex is found (see also Van der Laan [1980]). Using a geometric interpretation of this algorithm Van der Laan and Talman [1980b] and Shamir [1979] developed a continuous-deformation algorithm for which the factor of incrementation can be of any size so that fast convergence can be obtained. All these more sophisticated algorithms were developed both on $S^{\mathrm{n}}$ and $\mathrm{R}^{\mathrm{n}}$.

Beside the development of new algorithms many topics closely related to the approximation of a fixed point have been studied. Since computational experience revealed that the computation time highly depends on the underlying triangulation, Saigal, Solow and Wolsey [1973] developed a 
rough measure for the efficiency of a triangulation of the unit cube in a fixed point algorithm. Todd [1976b, 1978a] proposed a more sophisticated measure. However, even within a reasonable class of regular congruent triangulations it is not known how the optimal triangulation according to this measure looks like.

Another important problem is to state convergence conditions for a fixed point algorithm applied on unbounded regions. Merrill [1971, 1972], Gould and Tolle [1975], Todd [1976a, 1978b, 1980], Saigal [1977c], Reiser [1978a, 1978b], Saigal and Todd [1978], Van der Laan [1980], and others gave conditions for the various algorithms. It appeared that these conditions depend both on the algorithm and the underlying triangulation.

Fixed point algorithms for specially structured problems were studied by Kojima [1978a, 1978b] and Todd [1978c, 1978d]. Acceleration techniques to obtain quadratic convergence were derived by Wolsey[1974], Saigal and Todd [1978], Todd [1978e] and Reiser [1978a, 1978c]. When the function is smooth, the convergence rate can be considerably improved by performing a Newton-step once in a while.

Finally, we mention the studies about the kind of path of simplices generated by a fixed point algorithm. These studies were done by Lemke and Grotzinger [1976], Eaves and Scarf [1976], Todd [1976c], Saigal [1976], Garcia and Gould [1978], Saari and Saigal [1979], Kojina [1980] and Van der Laan [1980]. In most of these papers the orientation of a simplex plays an important role. The orientation theory was introduced by Shapley [1974] for bimatrix games.

Survey papers about fixed point algorithms and related topics are those of Gould and Tolle [1974], Eaves [1976], Saigal [1977b], and Allgower and Georg [1980]. We also refer to the books of Todd [1976a] and Lüthi [1976]. In this monograph a new measure for the efficiency of a triangulation of a convex subset is introduced. Within an attractive class of so-called "fixed point triangulations" of $R^{n}$ we calculate the optimal one according to this measure. It will appear that this triangulation of $\mathrm{R}^{\mathrm{n}}$ is a generalization of the equilateral triangulation of $R^{2}$. Also, a new triangulation of the affine hull of $\mathrm{S}^{\mathrm{n}}$ is proposed having the same measure in the above sense as the optimal triangulation of $R^{n}$. Further, a variable dimension algorithm is developed to compute a fixed point of a continuous function (or mapping) from the product space of unit simplices into itself. For this purpose we need a triangulation which depends on the starting 
point of the algorithm. Moreover, based on this algorithm a class of variable dimension algorithms on $\mathrm{R}^{\mathrm{n}}$ is developed. The two extreme cases of this class are of considerable interest. The first one is the basic algorithm of Van der Laan and Talman [1979b] whereas the other extreme case has the attractive property that fast movements in all directions can be made. The latter case can also be utilized to approximate a connected set of fixed points. Moreover, some new convergence conditions are discussed. Finally, three different geometric interpretations of the variable dimension algorithms are given.

This monograph is organized as follows.

In chapter 2 some preliminaries are given and the fixed point theorems of Brouwer and Kakutani are proved. Also some triangulations of $R^{n}$ and $S^{n}$ are discussed.

Chapter 3 is devoted to applications of fixed point theorems. The noncooperative $\mathrm{N}$-person game is treated and, after stating a sufficient condition for the existence of a fixed point on unbounded regions, the constrained optimization problem and the nonlinear complementarity problem are discussed.

Chapter 4 describes the algorithms of Kuhn [1968] and Eaves [1971], and the more sophisticated algorithms of Merrill [1971, 1972] and Van der Laan and Talman [1979a, 1979b]. Also vector labelling is introduced in this chapter.

Chapter 5 discusses some known measures for the efficiency of a triangulation for use in a fixed point algorithm and introduces a new measure. This measure is based on the ratio of the average number of grid points per unit cube and the accuracy of an approximate fixed point yielded by the triangulation. Within a reasonable class of triangulations of $\mathrm{R}^{\mathrm{n}}$ the optimal one is calculated and some of its properties are derived. For the affine hull of $\mathrm{s}^{\mathrm{n}}$ a new triangulation is proposed having the same nice properties. Computational results will confirm the superiority of the new triangulations.

Chapter 6 introduces a variable dimension fixed point algorithm on the product space $\mathrm{S}$ of $\mathrm{N}$ unit simplices. The triangulation of $\mathrm{S}$ will be defined in relation to the arbitrarily chosen starting point. As with Van der Laan and Talman's basic algorithm on $s^{n}$, the algorithm generates a unique path of adjacent simplices of variable dimension. However, the number of labels (equations) exceeds the dimension of $S$ by $N$. Whereas all 
other restart algorithms terminate when a completely labelled simplex is found, our restart algorithm terminates as soon as some special subset of labels is found where the algorithm's terminal simplex need not be fulldimensional. The dimension of this simplex, however, has no influence on the accuracy of the approximation. We will apply the algorithm to the noncooperative $\mathrm{N}$-person game.

In chapter 7 a class of variable dimension fixed point algorithms on $\mathrm{R}^{\mathrm{n}}$ is developed using the ideas of the algorithm presented in chapter 6 , by considering $\mathrm{R}^{\mathrm{n}}$ as the product space of $\mathrm{N}$ lower-dimensional Euclidean spaces. Both for integer and vector labelling new convergence conditions are discussed. Much attention will be paid to the extreme case $\mathrm{N}=\mathrm{n}$. We will indicate how for integer labelling in case $\mathrm{N}=\mathrm{n}$ the algorithm can be utilized to approximate a connected set of fixed points. For vector labelling we will prove that Merrill's condition is sufficient to guarantee convergence in case $\mathrm{N}=\mathrm{n}$ and also for the case $\mathrm{N}=1$ if the optimal triangulation derived in chapter 5 underlies the algorithm. Furthermore, an application to the Borsuk-Ulam theorem is discussed, cf. Todd and Wright [1979]. At the end of chapter 7 some computational experience is given. Our algorithm is also compared with the method of Reiser who recently developed for the nonlinear complementarity problem an integer labelling algorithm closely related to our case $\mathrm{N}=\mathrm{n}$.

Finally, chapter 8 gives three different geometric interpretations of the algorithms presented in the chapters 6 and 7. By these interpretations the algorithms can be viewed to trace zeroes of a piecewise linear homotopy function defined on a set being the convex hull of $S$ (or $R^{n}$ ) on a natural level and an artificial set on an additional level. 
CHAPTER 2

PRELIMINARIES

2.1. INTRODUCTION.

In 1910 L.E.J. Brouwer proved that any continuous function from a nonemptir, convex, compact subset of $R^{n}$ into itself has at least one fixed point. In this chapter we give a constructive proof of Brouwer's theorem by using the famous lemma of Sperner. In most fixed point algorithms the ideas of Sperner's result are fundamental. In Sperner's lemma we need the concept of a triangulation of a convex set. Section 2 gives the definitions of a triangulation and a subdivision. In section 3 both Sperner's lemma and Brouwer's theorem are proved. Section 4 discusses the fixed point theorem of Kakutani. This theorem generalizes Brouwer's theorem to upper semicontinuous point to set mappings. Finally, special triangulations of $R^{n}$ and the unit simplex are treated in the sections 5 and 6 respectively.

2.2. TRIANGULATIONS AND SUBDIVISIONS.

Throughout this monograph $\mathrm{R}^{\mathrm{n}}$ will denote the $\mathrm{n}$-dimensional Euclidean space

$$
R^{n}=\left\{\left(x_{1}, \ldots, x_{n}\right)^{\top} \mid x_{i} \text { real for } i=1, \ldots, n\right\}
$$

endowed with the usual metric $\rho(x, y)=\|x-y\|$ where

$$
\|x\|=\left\{\sum_{i=1}^{n} x_{i}^{2}\right\}^{\frac{1}{2}} .
$$

The i-th unit vector of $R^{n}$ will be denoted by $e(i), i=1, \ldots, n$, and the nonnegative orthant of $R^{n}$ by $R_{+}^{n}$, i.e.

$$
R_{+}^{n}=\left\{x \in R^{n} \mid x \geq 0\right\}
$$


The affine hull of an $m$-dimensional convex subset $C$ of $R^{n}$ is the set aff $C$ defined by

$$
\text { aff } C=\left\{y \mid y=\sum_{i=1}^{m+1} \lambda_{i} x^{i} \text { where } \sum_{i=1}^{m+1} \lambda_{i}=1 \text { and } x^{i} \in C, 1 \leq i \leq m+1\right\}
$$

Note that $\operatorname{dim}$ aff $\mathrm{C}=\operatorname{dim} \mathrm{C}$. In the sequel the interior and the boundary of $C$, to be denoted by int $C$ and bd $C$ respectively, are always taken with respect to aff $\mathrm{C}$.

We say that $t+1$ points $w^{1}, \ldots, w^{t+1}$ of $R^{n}$ are affinely independent if $\sum_{i=1}^{t+1} \lambda_{i} w^{i}=0$ and $\sum_{i=1}^{t+1} \lambda_{i}=0$ imply $\lambda_{i}=0, i=1, \ldots, t+1$. Note that this definition implies $t \leq n$.

Finally, the convex hull of $m$ points $w^{1}, \ldots, w^{m}$ of $R^{n}$ is the set $w$ defined by

$$
\mathrm{W}=\left\{\mathrm{y} \in \mathrm{R}^{\mathrm{n}} \mid \mathrm{y}=\sum_{i=1}^{\mathrm{m}} \lambda_{i} \mathrm{w}^{\mathrm{i}} \text { such that } \sum_{i=1}^{\mathrm{m}} \lambda_{i}=1 \text { and } \lambda_{i} \geq 0, i=1, \ldots, \mathrm{m}\right\}
$$

DEFINITION 2.2.1. If $\mathrm{w}^{1}, \ldots, \mathrm{w}^{t+1}$ are $t+1$ affinely independent points of $\mathrm{R}^{\mathrm{n}}$, then the convex hull of these points is called a $t$-simplex or $t$-dimensional simplex with vertices $w^{1}, \ldots, w^{t+1}$. Such a simplex is denoted by $\sigma$ or $\sigma\left(w^{1}, \ldots, w^{t+1}\right)$.

DEFINITION 2.2.2. A $k$-simplex $\tau$ is a face of a $t$-simplex $\sigma(k \leq t)$ if all vertices of $\tau$ are vertices of $\sigma$. If $k=t-1$ we call $\tau$ a facet of $\sigma$. If $y$ is the vertex of $\sigma$ which is not a vertex of the facet $\tau$ of $\sigma$, we say that $\tau$ is the facet of $\sigma$ opposite the vertex $y$.

DEFINITION 2.2.3. Two different simplices $\sigma_{1}$ and $\sigma_{2}$ are adjacent if they share a common facet or if one of them is a facet of the other.

Observe that in the last definition $\sigma_{1}$ and $\sigma_{2}$ have the same dimension if they share a common facet.

In the following $C$ denotes an m-dimensional convex subset of $R^{n}$.

DEFINITION 2.2.4. A collection $\mathrm{G}$ of m-simplices is a triangulation of $\mathrm{C}$ if

i) $C$ is the union of all simplices in $G$

ii) the intersection of two simplices in $G$ is either empty or a common face 
iii) Each facet either belongs to bd $C$ and is a facet of just one simplex of $G$ or does not belong to bd $C$ and is a facet of exactly two simplices of $\mathrm{G}$.

The following equivalent definition is sometimes useful to prove that a collection of simplices is a triangulation of a convex set.

DEFINITION 2.2.5. A collection $\mathrm{G}$ of m-simplices is a triangulation of $\mathrm{C}$ if the relative interiors of the faces of all the simplices in $G$ partition $C$ and if each compact subset of $C$ contains a finite number of them.

The proof that both definitions are equivalent follows from the fact that each simplex is partitioned by the relative interiors of its faces. observe that there are only m-simplices in $G$ and that if the set $C$ is not closed the number of simplices in $G$ is infinite. So, for example let $C=(0,1]$ and let $F$ be the collection of simplices $\sigma\left(w^{1}, w^{2}\right)$ in $R$ such that $\mathrm{w}^{1}=2^{-(\mathrm{k}+1)}$ and $\mathrm{w}^{2}=2^{-\mathrm{k}}, \mathrm{k}=0,1,2, \ldots$ Clearly, $\mathrm{F}$ is a triangulation of the set $C$. However, the union of the elements of $F$ and the zerodimensional simplex $\sigma_{0}\left(w^{1}\right)$ with $w^{1}=0$ is not a triangulation of $[0,1]$ since $\sigma_{0}$ is not 1-dimensional. Note that $\mathrm{F}$ consists indeed of an infinite number of elements.

We are not assuming that a triangulation is locally finite in the sense that each $\mathrm{x} \in \mathrm{C}$ has a neighbourhood meeting only a finite number of simplices of G (see e.g. Todd [1976a], Bárány [1979] and Eaves [1976]). In chapter 8 triangulations will appear which are not locally finite. A concept more general than a triangulation into simplices is a subdivision into polyhedra.

DEFINITION 2.2.6. A polyhedron $\sigma$ is the convex hull of a finite number of points of $\mathrm{R}^{\mathrm{n}}$. Moreover, let $\tau$ be a subset of $\sigma$. If for any $\mathrm{p} \in \tau$ and any $\mathrm{x}, \mathrm{y} \in \sigma$ such that $\mathrm{p}=\lambda \mathrm{x}+(1-\lambda) \mathrm{y}$ for some $\lambda, 0<\lambda<1$, holds that $\mathrm{x}, \mathrm{y} \in \tau$, then $\tau$ is called a face of $\sigma$. If $\operatorname{dim} \tau=\operatorname{dim} \sigma-1$ we call $\tau$ a facet of $\sigma$.

Now we can define a subdivision of $\mathrm{C}$.

DEFINITION 2.2.7. A collection M of m-dimensional polyhedra is a subdivision of $\mathrm{C}$ if 
i), $C$ is the union of all polyhedra in $M$

ii) the intersection of two polyhedra is either empty or a common face. and

iii) Each facet of a polyhedron either belongs to bd $C$ and is a facet of just one polyhedron of $\mathrm{M}$ or does not belong to bd $\mathrm{C}$ and is a facet of exactly two polyhedra of $M$.

Note that every triangulation is a subdivision. Figure $2.2 .1 \mathrm{a}$ is an example which gives neither a subdivision nor a triangulation, figure $2.2 .1 \mathrm{~b}$ is an example which givew a subdivision but not a triangulation, whereas figure 2.2.2 is an example of a triangulation.

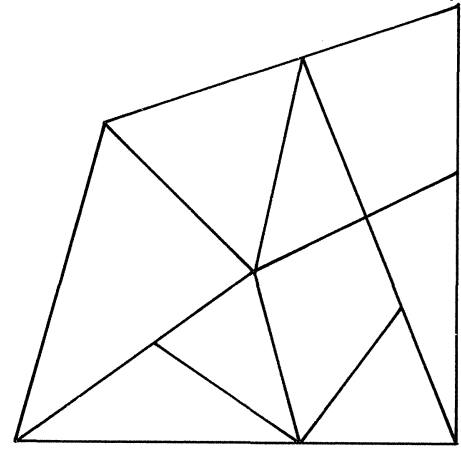

Figure 2.2.1a.

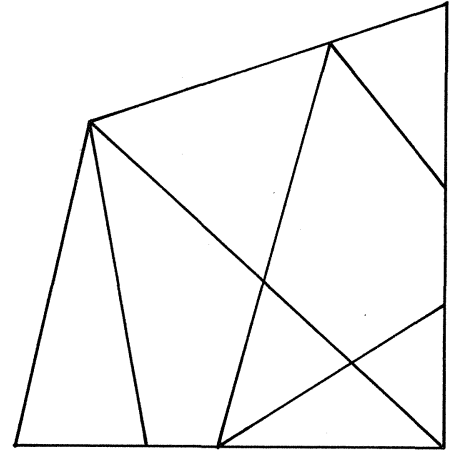

Figure 2.2.1b.

The proof of the next two theorems can be found in Spanier [1966]. Let $M$ be a subdivision (triangulation) of an m-dimensional convex set $C$ of $R^{n}$.

THEOREM 2.2.8. If the subdivision $M$ is locally finite then condition iii) follows from $i)$ and $i i)$.

THEOREM 2.2.9. Let $\mathrm{D}$ be an (m-1)-dimensional subset in the boundary of $\mathrm{C}$ such that $D$ is equal to the intersection of $C$ and the affine hull of $D$. Then $D$ is subdivided into (m-1)-dimensional polyhedra (simplices) which are containted in $\mathrm{D}$ are facets of polyhedra (simplices) in $\mathrm{M}$. 
These features are illustrated in figure 2.2.2. In this figure we have that the facet $\tau_{1}$ is a facet of both $\sigma_{1}$ and $\sigma_{2}$ whereas the boundary-facet $\tau_{2}$ is a facet of only $\sigma_{3}$. Moreover, let $D$ be the convex hull of the points $A$ and $B$. Then $D$ is triangulated (or subdivided) into three 1 -dimensional simplices $\tau_{2}, \tau_{3}$ and $\tau_{4}$.

To compare triangulations or subdivisions it is interesting to know what the largest distance between two points in the same simplex (or polyhedron) is.

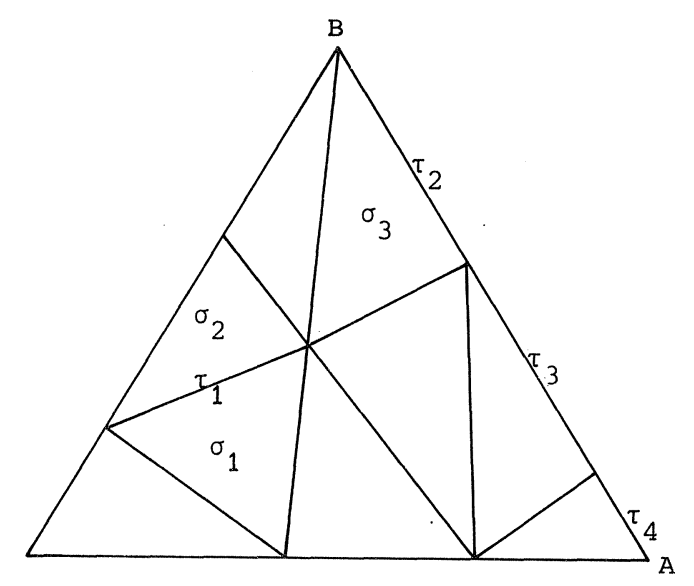

Figure 2.2.2. Illustration of condition iii) and theorem 2.2.9.

DEFINITION 2.2.10. The mesh of a subdivision $M$ of $C$, to be denoted by mesh $M$, is defined by

$$
\operatorname{mesh} M=\sup _{\sigma \in M}\{\operatorname{diam} \sigma\},
$$

where diam $\sigma=\max \{\|x-y\| \mid x, y \in \sigma\}$.

If $M$ is a triangulation, we have the following corollary.

COROLLARY 2.2.11. Let $G_{1}$ be the set of all one-faces of the simplices of a triangulation $\mathrm{G}$ of $\mathrm{C}$. Then

$$
\operatorname{mesh} G=\sup _{\tau \in G_{1}}\{\text { diam } \tau\}
$$


This result follows from the fact that diam $\sigma\left(w^{1}, \ldots, w^{t+1}\right)$ is equal to $\max _{i, j}\left\{\left\|w^{i}-w^{j}\right\|\right\}$ and the fact that the convex hull of two arbitrarily chosen vertices of a simplex is a one-face of that simplex. Note that the latter is not always true for a subdivision.

2.3. BROUWER'S THEOREM AND SPERNER'S LEMMA.

In this section we give a constructive proof of Brouwer's theorem. In this proof the lemma of Sperner [1928] plays a very crucial role.

Arguments in the proof are used in fixed point algorithms to be discussed in the later chapters. The idea is to triangulate the compact convex set on which we want to compute a fixed point. Then each vertex is labelled with one of the integers $1, \ldots, \mathrm{m}+1$ where $\mathrm{m}$ is the dimension of the set. This labelling is such that if the vertices of an m-simplex carry all the labels $1, \ldots, \mathrm{m}+1$, each point of the simplex is an approximate fixed point. By Sperner's lemma there always exists at least one such a simplex. If we take now a sequence of triangulations with mesh tending to zero, then there exists at least one subsequence such that the vertices of the simplex having all labels converge to a singleton. This point is then a fixed point as will be proved at the end of this section. More precisely, let $G$ be a triangulation of the m-dimensional convex subset $C$ of $R^{n}$ and assume that each vertex of the triangulation is labelled with one of the integers of the set $I_{m+1}=\{1, \ldots, m+1\}$. We call the set of labels of the vertices of a simplex the label set of the simplex.

DEFINITION 2.3.1. An $\mathrm{m}$-simplex in $\mathrm{G}$ is completely labelled if the $\mathrm{m}+1$ vertices of the simplex carry a different label, i.e. if the label set of the simplex is equal to $I_{m+1}$.

DEFINITION 2.3.2. The n-dimensional unit simplex is the set $s^{n}$ defined by

$$
S^{n}=\left\{x \in R_{+}^{n+1} \mid \sum_{i=1}^{n+1} x_{i}=1\right\} .
$$

The i-th boundary of $s^{n}$, to be denoted by $s_{i}^{n}$, is the set

$$
s_{i}^{n}=\left\{x \in s^{n} \mid x_{i}=0\right\} \quad i=1, \ldots, n+1
$$


Observe that $S^{\mathrm{n}}$ is indeed a simplex and that the vertices of $S^{\mathrm{n}}$ are the unit vectors $e(i), i=1, \ldots, n+1$. Moreover, $s_{i}^{n}$ is the facet of $s^{n}$ opposite to $e(i)$.

DEFINITION 2.3.3. For a given triangulation of $\mathrm{s}^{\mathrm{n}}$, a labelling is called proper if any vertex belonging to $s_{i}^{n}$ has a label different from $i$, $i=1, \ldots, n+1$.

LEMMA 2.3.4. (SPERNER). Let $G$ be some triangulation of $S^{n}$. Assume that $S^{n}$ is properly labelled with respect to $G$. Then there exists an odd number of completely labelled simplices in $G$ and hence $G$ has at least one completely labelled simplex.

PROOF. We follow the proof of Todd [1976a, pp. 12 and 13]. The proof is trivial for $\mathrm{n}=0$. By induction on $\mathrm{n}$ the lemma will be proved. So, assume the lemma is true for $n-1$ and let the vertices of a triangulation $G$ of $s^{n}$ be properly labelled. Furthermore, let $\mathrm{F}$ be the collection of facets of the simplices in $G$ whose vertices carry all the labels $1, \ldots, n$. Next, let $A$ be the set of completely labelled simplices in $G$ and let $B$ be the set of simplices in $G$ whose vertices carry the labels $1, \ldots, n$ but not $n+1$. Clearly each simplex in $A$ has just one facet in $F$ whereas each simplex in $B$ has exactly two facets in $\mathrm{F}$.

If $D$ is the set of facets of $F$ in the boundary of $s^{n}$ and $E$ is the set of facets of $F$ not in the boundary of $s^{n}$, then

$$
|A|+2|B|=|D|+2|E|
$$

where $|x|$ denotes the number of elements in the set $x$. To show that $|A|$ is odd we will show that $|D|$ is odd. Consider now the collection $G^{\prime}$ of (n-1)-simplices of the triangulation $G$ that are facets of $n$-simplices and lie in $S_{n+1}^{n}$. By theorem 2.2.9 $G^{\prime}$ is a triangulation of $s_{n+1}^{n}$. Moreover, there is a one-to-one correspondence between $s_{n+1}^{n}$ and $s^{n-1}$ since $x_{n+1}=0$ for any $x \in S_{n+1}^{n}$. Because of the proper labelling of the vertices of $G^{\prime}$, we obtain from the induction hypothesis that the number of completely labelled (n-1)-simplices in $G^{\prime}$ is odd. But this number is equal to $|D|$. Hence $|A|$ is also odd which completes the proof. 
We are now ready to prove Brouwer's theorem which says that a continuous function from a compact; convex subset of $R^{n}$ into itself has at least one point fixed.

THEOREM 2.3.5. (BROUWER'S THEOREM). Let $C$ be a compact, convex subset of $R^{n}$. Then any continuous function $\mathrm{f}: \mathrm{C} \rightarrow \mathrm{C}$ has at least one point $\mathrm{x}^{*}$ such that $f\left(x^{*}\right)=x^{*}$.

PROOF. Without loss of generality we can restrict ourselves to continuous functions from $s^{\mathrm{n}}$ into itself. To prove that any continuous function from $S^{n}$ into itself has at least one fixed point, let $\left\{G_{k}, k=1,2, \ldots\right\}$ be a sequence of triangulations of $\mathrm{s}^{\mathrm{n}}$ such that $\varepsilon_{\mathrm{k}}=\operatorname{mesh} \mathrm{G}_{\mathrm{k}} \rightarrow 0$ if $\mathrm{k} \rightarrow \infty$. Each point $\mathrm{y}$ in $\mathrm{S}^{\mathrm{n}}$ receives now an integer label $\ell(\mathrm{y})$ defined by

$$
\ell(y)=\min \left\{j \mid f_{j}(y) \leq y_{j} \text { and } y_{j}>0\right\} .
$$

Clearly, with respect to any $G_{k}$ this labelling rule is proper. Note that $\ell(y)$ is independent of the triangulation $G_{k_{2}}$. By Sperner's lemma, there is at least one completely labelled simplex $\sigma_{k}$ in $G_{k}, k=1,2, \ldots$. Let $y^{i}(k)$ be the vertex of $\sigma_{k}$ with $l\left(y^{i}(k)\right)=i, i=1, \ldots, n+1$. Then for some subsequence $\left\{k_{j}, j=1,2, \ldots\right\}$ of indices with $k_{j} \rightarrow \infty$ if $j \rightarrow \infty$, we have that the subsequence $\left\{\mathrm{y}^{1}\left(\mathrm{k}_{j}\right), j=1,2, \ldots\right\}$ converges to a point $\mathrm{x}^{*}$ in $\mathrm{s}^{\mathrm{n}}$. Since $\varepsilon_{k} \rightarrow 0$ if $k \rightarrow \infty$, it follows immediately that $y^{i}\left(k_{j}\right) \rightarrow x^{*}$ if $j \rightarrow \infty$, for all $i$. From the labelling rule we know that $f_{i}\left(y^{i}\left(k_{j}\right)\right) \leq y_{i}\left(k_{j}\right)$ for all $i$ and $j$. Hence, by the continuity of the function $f, f_{i}\left(x^{*}\right) \leq x_{i}^{*}$ for all $i$. Since, however,

$$
\sum_{i=1}^{n+1} f_{i}\left(x^{*}\right)=\sum_{i=1}^{n+1} x_{i}^{*}
$$

it follows that $f_{i}\left(x^{*}\right)=x_{i}^{*}$ for all $i, i . e . x^{*}$ is a fixed point of $f$.

The idea of a fixed point algorithm is to generate a sequence of completely labelled simplices $\sigma_{k}, k=1,2, \ldots$, with diam $\sigma_{k} \rightarrow 0$ as $k \rightarrow \infty$.

2.4. KAKUTANI'S THEOREM.

Before stating Kakutani's fixed point theorem, which is a generalization of Brouwer's theorem, we need some definitions, 
cf. Berge [1966].

DEFINITION 2.4.1. Let $\phi$ be a point to set mapping from a set $C$ in $R^{n}$ to the set of subsets of $R^{P}$. Then $\phi$ is upper semi-continuous at the point $x^{*}$ if

i) $\phi\left(x^{*}\right)$ is compact

and

ii) for any open set $D$ containing $\phi\left(x^{*}\right)$ there exists an open neighbourhood $U$ of $x^{*}$ such that $\phi(x) \subset D$ for all $x \in U$.

The mapping $\phi$ is called upper semi-continuous (u.s.c.) on $\mathrm{C}$ if $\phi$ is upper semi-continuous at any point of $C$.

The following result follows immediately from this definition, cf. Todd [1976a].

COROLLARY 2.4.2. If $\phi$ is an u.s.c. mapping from $C$ to the set of subsets of $R^{p}$, then for any sequence $\left\{x_{k}\right\}$ in $C$ with limit $x^{*}$ and any sequence $\left\{\mathrm{y}_{\mathrm{k}}\right\}$ in $\mathrm{R}^{\mathrm{p}}$ with limit $\mathrm{y}^{*}$ such that $\mathrm{y}_{\mathrm{k}} \in \phi\left(\mathrm{x}_{\mathrm{k}}\right)$ for all $\mathrm{k}$, we have

$$
y^{*} \in \phi\left(x^{*}\right)
$$

Clearly, upper semi-continuity of a mapping $\phi$ from a compact, convex set $C$ to the set of nonempty subsets of $C$ is not sufficient for the existence of a point $\mathrm{x}^{*}$ in $\mathrm{C}$ such that $\mathrm{x}^{*} \epsilon \phi\left(\mathrm{x}^{*}\right)$. We call such a point a fixed point of the mapping $\phi$. However, if $\phi(x)$ is convex for all $x \in C$, then we have the following theorem due to Kakutani [1941] (see also Von Neumann [1937]).

THEOREM 2.4.3. (KAKUTANI). Let $\mathrm{C}$ be a compact, convex m-dimensional subset of $R^{n}$ and let $\phi$ be an upper semi-continuous mapping from $C$ to the set of non-empty, convex subsets of $C$. Then there is at least one point $x^{*}$ in $C$ such that $\mathrm{x}^{*} \in \phi\left(\mathrm{x}^{*}\right)$.

To prove the theorem we need the concept of a piecewise linear approximation to a mapping with respect to a triangulation. 
DEFINITION 2.4.4. Let $G$ be a triangulation of a convex m-dimensional subset $C$ of $R^{n}$ and $\phi$ a mapping from $C$ to the set of subsets of $R^{p}$. For any vertex $w$ of the triangulation $G$ choose some vector $f(w) \in \phi(w)$. Let $\mathrm{x}$ be an arbitrarily chosen point of $c$. Then $\mathrm{x}$ can be represented by

$$
x=\sum_{i=1}^{m+1} \lambda_{i} w^{i}
$$

for vertices $w^{1}, \ldots, w^{m+1}$ of a simplex $\sigma\left(w^{1}, \ldots, w^{m+1}\right)$ in $G$ and numbers $\lambda_{i} \geq 0$ with $\Sigma_{i=1}^{m+1} \lambda_{i}=1$, where $\lambda_{i}$ and $w^{i}$ are unique for all $i$ with $\lambda_{i}>0$. Then the function $f: C \rightarrow R^{p}$ defined by

$$
f(x)=\sum_{i=1}^{m+1} \lambda_{i} f\left(w^{i}\right)
$$

is called a piecewise linear approximation to the mapping $\phi$ with respect to the triangulation $\mathrm{G}$.

It is immediately verified that $f$ is continuous.

PROOF (OF THEOREM 2.4.3). We follow here the proof of Eaves [1971]. Let $\mathrm{C}^{\prime}$ be an $\mathrm{m}$-simplex containing $\mathrm{C}$, and let $\mathrm{c}$ be an interior point of $\mathrm{C}$. Define now the mapping $\bar{\phi}$ from $C^{\prime}$ to the set of non-empty, compact, convex subsets of $C^{\prime}$ by

$$
\begin{array}{ll}
\bar{\phi}(\mathrm{x})=\phi(\mathrm{x}) & \mathrm{x} \in \text { int } \mathrm{C}, \\
\bar{\phi}(\mathrm{x})=\operatorname{conv}(\phi(\mathrm{x}) \cup\{\mathrm{c}\}) & \mathrm{x} \in \mathrm{bd} \mathrm{C}
\end{array}
$$

and

$$
\bar{\phi}(x)=\{c\} \quad x \in C^{\prime} \backslash C
$$

where conv ( $A \cup B$ ) denotes the convex hull of the sets $A$ and $B$. It is not difficult to show that $\bar{\phi}$ is an u.s.c. mapping on $C^{\prime}$.

Suppose that $\bar{\phi}$ has a fixed point $x^{*}$. Clearly $x^{*} \epsilon$ C. If $x^{*} \epsilon$ int $C$ then $x^{*} \epsilon \phi\left(x^{*}\right)$ and hence $x^{*}$ is also a fixed point of $\phi$. If $x^{*} \epsilon$ bd $C$, then $x^{*}=\lambda c+(1-\lambda) y$ for some $y \in \phi\left(x^{*}\right)$ and $\lambda, 0 \leq \lambda \leq 1$. Since $c \in$ int $c$, we have that a positive $\lambda$ gives the contradiction $x^{*} \epsilon$ int c. Hence $\lambda=0$ and $y=x^{*}$ so that again $x^{*} \epsilon \phi\left(x^{*}\right)$. Therefore if $x^{*}$ is a fixed point of 
$\bar{\phi}, \mathbf{x}^{*}$ is also a fixed point of $\phi$. To prove that $\bar{\phi}$ has at least one fixed point, let $\left\{G_{k}\right\}$ be a sequence of triangulations of $C^{\prime}$ with mesh $G_{k}=\varepsilon_{k} \rightarrow 0$ as $\mathrm{k} \rightarrow \infty$. Moreover, let $\mathrm{f}_{\mathrm{k}}$ be a piecewise linear approximation to $\bar{\phi}$ with respect to $G_{k}, k=1,2, \ldots$. By Brouwer's theorem, $f_{k}$ has a fixed point, say $\mathrm{x}^{\mathrm{k}}$. Also there is at least one simplex $\sigma_{\mathrm{k}}$ with vertices $\mathrm{y}^{1}(\mathrm{k}), \ldots, \mathrm{y}^{\mathrm{m}+1}(\mathrm{k})$ containing $\mathrm{x}^{\mathrm{k}}$. Hence, for unique nonnegative numbers $\lambda_{1}^{\mathrm{k}}, \ldots, \lambda_{\mathrm{m}+1}^{\mathrm{k}}$ with sum equal to one,

$$
x^{k}=f_{k}\left(x^{k}\right)=\sum_{i=1}^{m+1} \lambda_{i}^{k} f_{k}\left(y^{i}(k)\right) \quad k=1,2, \ldots
$$

Since $C^{\prime}$ is compact there is a subsequence $\left\{k_{j}, j=1,2, \ldots\right\}$ of integers such that $x^{k} \rightarrow x^{*}, \lambda_{i}{ }^{k} \rightarrow \lambda_{i}^{*}$ and $f_{k_{j}}\left(y^{i}\left(k_{j}\right)\right) \rightarrow f^{i}$ for all $i \in I_{m+1}$ as $j \rightarrow \infty$. Clearly, $x^{*} \in C^{\prime}, \lambda_{i}^{*} \geq 0$ and $\sum_{i=1}^{m+1} \lambda_{i}^{*}=1$. Because of corollary 2.4 .2 we also know that $f^{i} \in \bar{\phi}\left(x^{*}\right), i=1, \ldots, m+1$. Taking $k=k_{j}$ in the equation above and letting $j \rightarrow \infty$, we obtain

$$
x^{*}=\sum_{i=1}^{m+1} \lambda_{i}^{*} f^{i} \text { with } \lambda_{i}^{*} \geq 0, \sum_{i=1}^{m+1} \lambda_{i}^{*}=1 \text { and } f^{i} \in \bar{\phi}\left(x^{*}\right) .
$$

Therefore, since $\bar{\phi}\left(\mathrm{x}^{*}\right)$ is convex, $\mathrm{x}^{*} \in \bar{\phi}\left(\mathrm{x}^{*}\right)$, which proves that $\bar{\phi}$ has indeec a fixed point.

All algorithms computing Kakutani-fixed points are based on the technique of piecewise linear approximation to the mapping with respect to a sequence of triangulations with mesh tending to zero.

2.5. SPECIAL TRIANGULATIONS OF $\mathrm{R}^{\mathrm{n}}$.

Although Brouwer was already concerned with the existence of triangulations, Freudenthal [1942] was the first who constructed a triangulation of $\mathrm{R}^{\mathrm{n}}$. This triangulation, called the $\mathrm{K}$ triangulation (or I triangulation), was rediscovered later by Tucker in 1945 (see Lefschetz [1949, page 140]) and yields the standard triangulation of the unit cube as given by Kuhn [1960]. In the sequel we denote by $I_{k}$ the set of integers

$$
I_{k}=\{1, \ldots, k\} \quad k=1,2, \ldots .
$$


DEFINITION 2.5.1. The $\mathrm{K}$ (or standard) triangulation of $\mathrm{R}^{\mathrm{n}}$ with grid size $\delta>0$ is the collection of all simplices $\sigma\left(\mathrm{y}^{1}, \pi\right)$ with vertices $y^{1}, \ldots, y^{n+1}$ such that

i) each component of $y^{1}$ is a multiple of $\delta$,

ii) $\pi=\left(\pi_{1}, \ldots, \pi_{n}\right)$ is a permutation of the elements of $I_{n}$ ' iii) $y^{i+1}=y^{i}+\delta e\left(\pi_{i}\right) \quad i=1, \ldots, n$.

Note that all components of $y^{i}, i=1, \ldots, n+1$, are a multiple of $\delta$. We refer the reader to Todd [1976a] for the proof that the collection of simplices defined above is indeed a triangulation of $R^{n}$. The collection of simplices such that $\mathrm{y}^{1}=0$ and $\delta=1$ gives the standard triangulation of the unit cube of $\mathrm{R}^{\mathrm{n}}$ in $\mathrm{n}$ ! simplices.

COROLLARY 2.5.2. The mesh of the $\mathrm{K}$ triangulation with gridsize $\delta$ is equal to $\delta \sqrt{ }$.

The result follows from the fact that diam $\sigma\left(\mathrm{y}^{1}, \pi\right)=\left\|\mathrm{y}^{1}-\mathrm{y}^{\mathrm{n}+1}\right\|=$ $\delta\|\mathrm{e}\|=\delta \sqrt{\mathrm{n}}$ for each simplex $\sigma\left(\mathrm{y}^{1}, \pi\right)$, where e denotes the vector $(1, \ldots, 1)^{\top}$. Another well-known triangulation is due to Merrill [1971,1972] and Eaves and Saigal [1972]. It is the $\mathrm{H}$ triangulation and is closely related to the $\mathrm{K}$ triangulation. The $\mathrm{H}$ triangulation with grid size $\delta>0$ is the collection of all simplices $\sigma\left(y^{1}, \pi\right)$ with vertices $y^{1}, \ldots, y^{n+1}$ such that
i) each component of $y^{1}$ is a multiple of $\delta$,
ii) $\pi=\left(\pi_{1}, \ldots, \pi_{n}\right)$ is a permutation of the elements of $I_{n}$ '
iii) $y^{i+1}=y^{i}+\delta \tilde{q}\left(\pi_{i}\right) \quad i=1, \ldots, n$,

where $\tilde{q}(j)$ is the $j$-th column of the $n \times n$ matrix

$$
\tilde{Q}=\left[\begin{array}{rrrrrrr}
-1 & 0 & \ldots & \ldots & \ldots & 0 \\
1 & -1 & & & & \vdots \\
0 & 1 & . & & & \vdots \\
\vdots & & & & & \vdots \\
\vdots & & & & & \vdots & \\
\vdots & & & & -1 & 0 \\
0 & \ldots & \ldots & 0 & 1 & -1
\end{array}\right]
$$


Also for this triangulation we have that the mesh is equal to $\delta \sqrt{ }$. Observe that the $\mathrm{H}$ triangulation can be viewed as a nonsingular transformation of the $\mathrm{k}$ triangulation with transformation matrix $\tilde{Q}$. Generally, we have the following definition.

DEFINITION 2.5.3. Let $A$ be a nonsingular $n \times n$ matrix. Then the $A K$ triangulation with gridsize $\delta>0$ is the collection of all simplices $\sigma\left(y^{1}, \pi\right)$ with vertices $y^{1}, \ldots, y^{n+1}$ such that

i) each component of $A^{-1} Y^{1}$ is a multiple of $\delta$,

ii) $\pi=\left(\pi_{1}, \ldots, \pi_{n}\right)$ is a permutation of the elements of the set $I_{n}$ ' iii) $y^{i+1}=y^{i}+\delta a\left(\pi_{i}\right) \quad i=1, \ldots, n$,

where $a(j)$ is the $j$-th column of the matrix $A$.

Equivalently we can say that the AK triangulation is the collection of all simplices $\sigma\left(y^{1}, \ldots, y^{n+1}\right)$ such that $\sigma\left(A^{-1} y^{1}, \ldots, A^{-1} y^{n+1}\right)$ is a simplex of the $\mathrm{K}$ triangulation. Note that for given permutation $\pi$ all simplices $\sigma\left(\mathrm{y}^{1}, \pi\right)$ are congruent to each other. Therefore an AK triangulation is said to be a regular triangulation. So, to compute the mesh of an AK triangulation we can restrict ourselves to the simplices $\sigma\left(y^{1}, \pi\right)$ with $y^{1}$ equal to the vector $\underline{0}=(0, \ldots, 0)^{\top}$.

COROLLARY 2.5.4. The mesh of the AK triangulation with grid size $\delta$ is equal to

$$
\operatorname{mesh} A K=\delta \max _{S \subset I_{n}}\left\|\sum_{j \in S} a(j)\right\|
$$

The corollary follows from the fact that

$$
\operatorname{diam} \sigma(\underline{0}, \pi)=\delta \max _{1 \leq i \leq k \leq n}\left\|\sum_{j=i}^{k} a\left(\pi_{j}\right)\right\| .
$$

Observe that the mesh of the AK triangulation goes to zero as $; \delta$ goes to zero. Moreover, by a shift of the grid any a priori chosen point $z$ can be made to be a grid point of the triangulation. To keep simplices $\sigma\left(y^{1}, \pi\right)$ of an AK triangulation in storage, we only need the first vertex $y^{1}$ and the permutation $\pi$. Because of these nice properties an AK triangulation is often used in fixed point algorithms. In most of these algorithms a path of 
adjacent simplices is generated starting with an a priori determined simplex and terminating with a simplex which yields an approximate fixed point.

Therefore we need a rule giving the representation of the $\mathrm{n}$-simplex adjacent to a simplex $\sigma\left(y^{1}, \pi\right)$ if one of the vertices of the latter, say $\mathrm{y}^{\mathrm{S}}$, is replaced. Note that from theorem 2.2.8 it follows that there is exactly one such simplex. Let $\sigma\left(w^{1}, \gamma\right)$ be the representation of the new simplex, then we have the following theorem.

THEOREM 2.5.5. If $\sigma_{1}=\sigma\left(y^{1}, \pi\right)$ and $\sigma_{2}$ are two adjacent $n$-simplices of the AK triangulation such that $\tau\left(y^{1}, \ldots, y^{s-1}, y^{s+1}, \ldots, y^{n+1}\right)$ is the common facet, then $\sigma_{2}=\sigma\left(w^{1}, \gamma\right)$ where

$$
\begin{aligned}
& w^{1}=y^{1}+\delta a\left(\pi_{1}\right) \text { and } \gamma=\left(\pi_{2}, \ldots, \pi_{n}, \pi_{1}\right) \quad \text { if } s=1 \\
& w^{1}=y^{1} \quad \text { and } \gamma=\left(\pi_{1}, \ldots, \pi_{s-2}, \pi_{s}, \pi_{s-1}, \ldots, \pi_{n}\right) \text { if } 2 \leq s \leq n \\
& w^{1}=y^{1}-\delta a\left(\pi_{n}\right) \text { and } \gamma=\left(\pi_{n}, \pi_{1}, \ldots, \pi_{n-1}\right) \quad \text { if } s=n+1 \text {. }
\end{aligned}
$$

PROOF. Obviously, $\sigma\left(w^{1}, \gamma\right)$ with $w^{1}$ and $\gamma$ defined above is an n-simplex of the AK triangulation. Moreover it is easy to see that for these $w^{1}$ and $\gamma$ holds that

$$
\sigma\left(w^{1}, \gamma\right) \cap \sigma\left(y^{1}, \pi\right)=\tau\left(y^{1}, \ldots, y^{s-1}, y^{s+1}, \ldots, y^{n+1}\right)
$$

Hence, $\sigma_{2}$ has the representation stated in the theorem.

Finally, we discuss in this section the so-called J- or "Union Jack" triangulation, a centrally symmetric triangulation due to Tucker (cf. also Lefschetz [1949, page 140]), Whitney [1959, page 358] and Todd [1977]. This triangulation with grid size $\delta>0$ is the collection of simplices $\sigma\left(y^{1}, \pi, s\right)$ with vertices $y^{1}, \ldots, y^{n+1}$ such that
i) the components of $y^{1}$ are odd multiples of $\delta$,
ii) $\pi$ is a permutation of the elements of $I_{n}$ '
iii) $s$ is a sign vector in $\mathrm{R}^{\mathrm{n}}$, i.e. $\mathrm{s}_{i} \in\{1,-1\}$,
iv) $y^{i+1}=y^{i}+\delta s_{\pi_{i}} e\left(\pi_{i}\right) \quad i=1, \ldots, n$. 
It is easy to see that the mesh of this triangulation is also equal to $\delta \sqrt{n}$. In the same way as above we can define the AJ triangulation for nonsingular $n \times n$ matrices $A$. Clearly we have

$$
\operatorname{mesh} A J=\delta \max _{S, T \subset I_{n}}\left\{\left\|\sum_{j \in S} a(j)-\sum_{i \in T} a(i)\right\|\right\},
$$

where $\delta$ is the grid size.

The $\mathrm{K}$-and $\mathrm{H}$ triangulations are illustrated for $\mathrm{n}=2$ in the figures $2.5 .1 \mathrm{a}$ and $\mathrm{b}$ respectively.

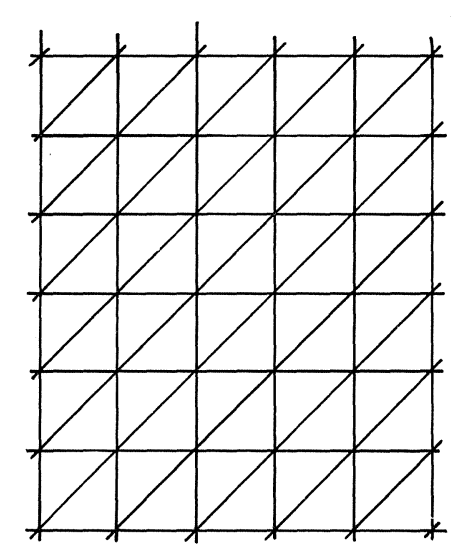

a. The $\mathrm{K}$ triangulation.

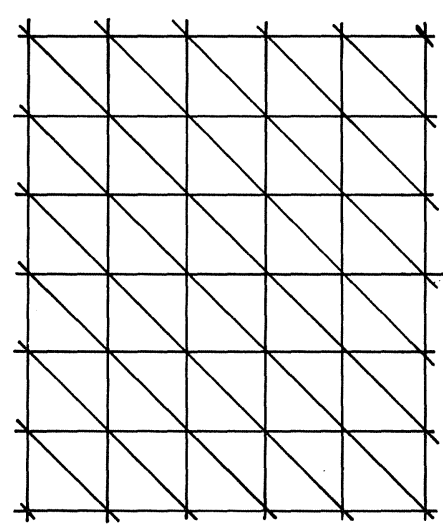

b. The $\mathrm{H}$ triangulation.

Figure 2.5.1.

In chapter 5 we shall see how the efficiency of a fixed point algorithm depends on the underlying triangulation. In the same chapter we propose a new triangulation that is in some sense optimal within a class of triangulations very suitable for fixed point algorithms.

2.6. TRIANGULATIONS OF $s^{\mathrm{n}}$.

In this section we triangulate the n-dimensional unit simplex $s^{n}$ on which many fixed point algorithms were developed (cf. Scarf [1967], Eaves [1971,1972], Kuhn and Mackinnon [1975], and Van der Laan and Talman [1979a]). Remember that Sperner's lemma was also given on $S^{n}$. Before discussing regular triangulations of $\mathrm{s}^{\mathrm{n}}$, we give the so-called iterated barycentric subdivision most familiar to topologists. For this 
triangulation Shapley [1973] introduced a fixed point algorithm. Since the triangulation yields long and skinny simplices, it is not very suitable for use in fixed point algorithms (see also chapter 5). The first barycentric subdivision of $s^{n}$ consists of $(n+1)$ : simplices $\sigma(\gamma)$ each associated with some permutation $\gamma$ of the elements of $I_{n+1}$. More precisely, $\sigma(\gamma)$ is the $n$-simplex with vertices $w^{1}, \ldots, w^{n+1}$ such that

$$
w^{i}=i^{-1} \sum_{j=1}^{i} e\left(\gamma_{j}\right) \quad i=1, \ldots, n+1 .
$$

In each following stage an $n$-simplex $\sigma\left(v^{1}, \ldots, v^{n+1}\right)$ is again barycentrally triangulated in $(n+1)$ : simplices $\sigma\left(\gamma \mid v^{1}, \ldots, v^{n+1}\right)$ with vertices $w^{1}, \ldots, w^{n+1}$ such that

$$
w^{i}=i^{-1} \sum_{j=1}^{i} v_{j}^{\gamma} \quad i=1, \ldots, n+1 .
$$

The first stage and partly the second stage are pictured in figure 2.6.1 for $n=2$. In this figure $\sigma$ is a simplex of the first stage and $\tau$ a simplex of the second stage.

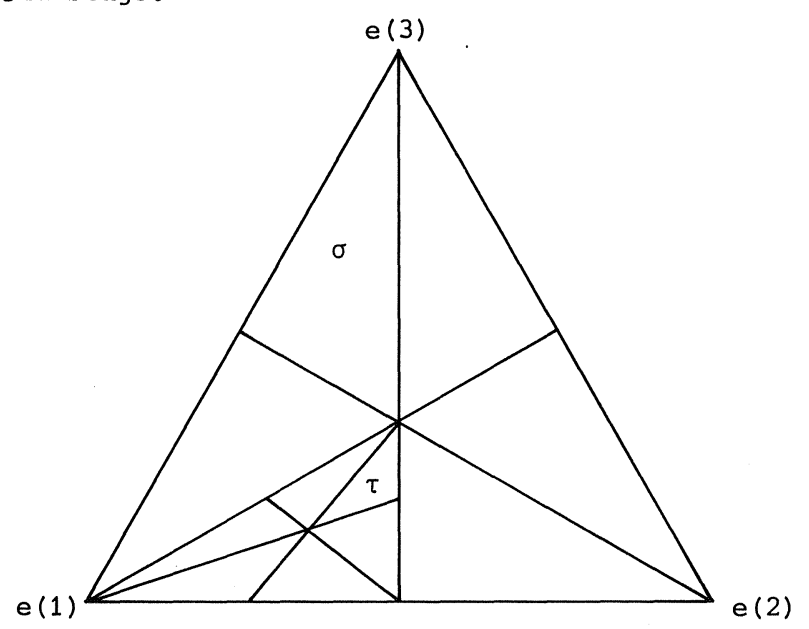

Figure 2.6.1. The iterated barycentric subdivision.

Closely related triangulations were proposed by zangwill [1977] and Stynes [1979]. Also these triangulations yield long and skinny simplices affecting the accuracy. Moreover, the replacement step of these triangulations are complicated and difficult to program. 
Now we discuss a triangulation of $S^{n}$ which is very popular in fixed point algorithms. This triangulation is referred to as the standard or $Q$ triangulation of $S^{n}$ and is closely related to the öK or $\mathrm{H}$ triangulation of $\mathrm{R}^{\mathrm{n}}$.

DEFINITION 2.6.1. The $\Omega$ (or standard) triangulation of $\mathrm{s}^{\mathrm{n}}$ with grid size $\mathrm{m}^{-1}$ ( $\mathrm{m}$ is a positive integer) is the collection of all simplices $\sigma\left(\mathrm{y}^{1}, \pi\right)$ with vertices $y^{1}, \ldots, y^{n+1}$ in $s^{n}$ such that

i) each component of $\mathrm{y}^{1}$ is a multiple of $\mathrm{m}^{-1}$,

ii) $\pi=\left(\pi_{1}, \ldots, \pi_{n}\right)$ is a permuation of the elements of $I_{n}$,

iii) $y^{i+1}=y^{i}+m^{-1} q\left(\pi_{i}\right) \quad i=1, \ldots, n$,

where $q(j)$ is the $j-$ th column of the $(n+1) \times n$ matrix

$$
Q=\left[\begin{array}{rrrrrrr}
-1 & 0 & . & . & . & . & 0 \\
1 & -1 & & & & & \cdot \\
0 & 1 & -1 & & & & \cdot \\
\cdot & & & \cdot & & & \cdot \\
\cdot & & & & \cdot & & \cdot \\
\cdot & & & & & -1 & \cdot \\
\cdot & & & & & 1 & -1 \\
0 & . & . & . & . & 0 & 1
\end{array}\right] \text {. }
$$

Observe that $q(i)=e(i+1)-e(i), i=1, \ldots, n, i . e . q(i)$ is the difference between two vertices of $\mathrm{S}^{\mathrm{n}}$. Actually, the $\mathrm{Q}$ triangulation can be seen as the transformation $Q$ of the $K$ triangulation of $R^{n}$, restricted to the set $S^{n}$. Note that the rank of 0 is $n$ and that $\Sigma_{i=1}^{n+1} q_{i j}=0, j=1, \ldots, n$. In the same way we can define the $\Omega \mathrm{J}$ triangulation with gridsize $\mathrm{m}^{-1}$. This triangulation is the collection of all simplices $\sigma\left(y^{1}, \pi, s\right)$ with vertices $y^{1}, \ldots y^{n+1}$ in $s^{n}$ such that

i) $y^{1}=e(1)+m^{-1} \sum_{j=1}^{n} \lambda_{j} q(j)$ for even nonnegative integers $\lambda_{j}$,

ii) $\pi$ is a permutation of the elements of $I_{n}$,

iii) $s$ is a sign vector in $R^{n}$,

iv) $y^{i+1}=y^{i}+m^{-1} s_{\pi_{i}} q\left(\pi_{i}\right), i=1, \ldots, n$. 
Both triangulations are illustrated for $\mathrm{n}=2$ and $\mathrm{m}=5$ in the figures 2.6.2a respectively $2.6 .2 \mathrm{~b}$.

In general, we obtain a regular triangulation of a $\mathrm{k}$-simplex $v^{1}, \ldots, v^{k+1}$, if we set $q(i)=v^{i+1}-v^{i}, i=1, \ldots, k$.

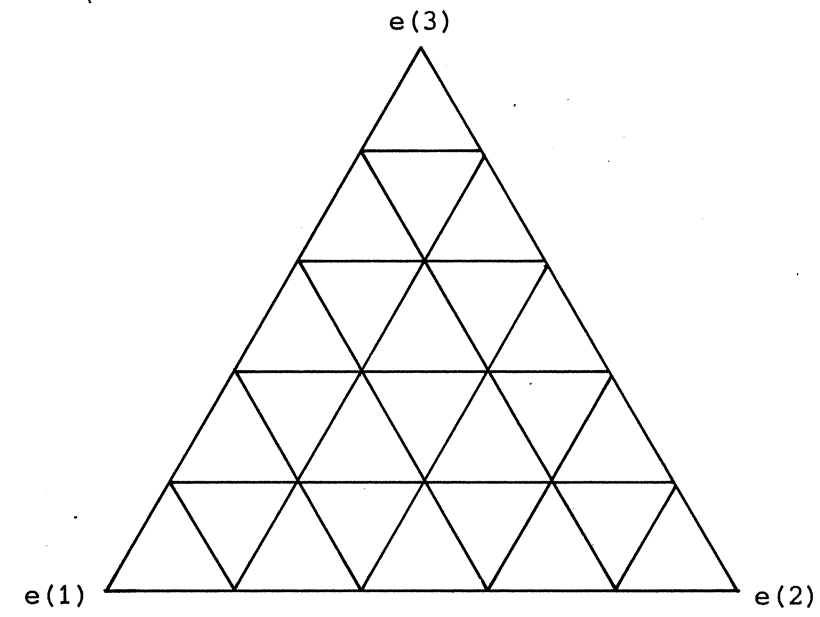

Figure 2.6.2a. The Q triangulation.

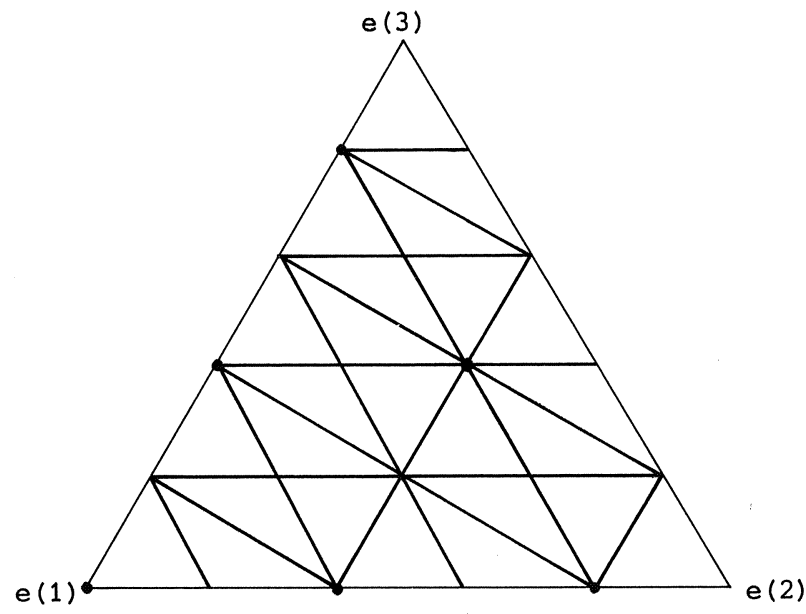

Figure 2.6.2b. The QJ triangulation. 
Clearly, the triangulations of $S^{n}$ can be extended directly to triangulations of the affine hull of $\mathrm{s}^{\mathrm{n}}$. This set will be denoted by $\mathrm{U}^{\mathrm{n}}$ and is given by

$$
U^{n}=\left\{x \in R^{n+1} \mid \sum_{i=1}^{n+1} x_{i}=1\right\}
$$

of course, m need not to be an integer, whereas the grid can be shifted to make an a priori chosen point $\mathrm{z}$ of $\mathrm{U}^{\mathrm{n}}$ to be a grid point of the triangulation. Moreover, any transformation $Q A$ of the $\mathrm{K}$ triangulation gives a triangulation of $U^{n}$ if $A$ is a nonsingular $n \times n$ matrix. Only in a few cases the simplices which cover $s^{n}$ form a triangulation of $s^{n}$. In chapter 5 we pay more attention to this matter. Note that the matrix 9 is not an orthogonal mapping so that simplices of the $\mathrm{K}$ triangulation of $\mathrm{R}^{\mathrm{n}}$ are deformed by the $\Omega$ transformation to $\mathrm{S}^{\mathrm{n}}$. Consequently, in general mesh $\mathrm{QAK}$ is not equal to mesh $\mathrm{AK}$.

LEMMA 2.6.2. Let $\mathrm{m}^{-1}$ be the grid size of the triangulation.

$$
\begin{aligned}
& \text { a) mesh } \Omega A K=m^{-1} \max _{S \subset I}\left\|\sum_{j \in S} Q a(j)\right\| \\
& \text { b) mesh } \mathrm{QAJ}=\mathrm{m}^{-1} \max _{\mathrm{S}, \mathrm{T} \subset I_{\mathrm{n}}}\left\|\sum_{j \in \mathrm{S}} \varrho \mathrm{a}(j)-\sum_{i \in \mathrm{T}} \Omega \mathrm{a}(i)\right\| \text {. }
\end{aligned}
$$

PROOF.

a) Let $z^{1}$ be a grid point of the triangulation. Then

$$
\text { mesh } \mathrm{QAK}=\max _{\sigma\left(z^{1}, \pi\right)} \max _{i \in I_{n+1}}\left\|z^{i}-z^{1}\right\|
$$

since the triangulation is regular, where $z^{i}$ denotes the $i$-th vertex of $\sigma\left(z^{1}, \pi\right)$. Since $z^{i}$ is equal to

$$
z^{i}=z^{1}+m^{-1} \sum_{j=1}^{i-1} Q a\left(\pi_{j}\right) \quad i=1, \ldots, n+1,
$$

we obtain

$$
\max _{\sigma\left(z^{1}, \pi\right)} \max _{i \in I_{n+1}}\left\|z^{i}-z^{1}\right\|=m^{-1} \max _{S \subset I_{n}}\left\|\sum_{j \in S} Q a(j)\right\| .
$$

b) Let $z^{1}$ be a grid point such that 


$$
z^{1}=e(1)+m^{-1} \sum_{j=1}^{n} \lambda_{j} q(j) \text { for even nonnegative integers } \lambda_{j} \cdot
$$

Then in the same way as in part a) we obtain

$$
\begin{aligned}
\operatorname{mesh} Q A J & =\max \underset{\left(z^{1}, \pi, s\right)}{\max _{i \in I_{n+1}}\left\|z^{i}-z^{1}\right\|=} \\
& =m^{-1} \max _{S, T \subset I_{n}}\left\|\sum_{j \in S} Q a(j)-\sum_{i \in T} \Omega a(i)\right\| .
\end{aligned}
$$

COROLLARY 2.6.3. Let $\mathrm{m}^{-1}$ be the grid size of the triangulation.

a) mesh $\mathrm{Q}=\mathrm{m}^{-1} \sqrt{\mathrm{n}+1}$ if $\mathrm{n}$ is odd and mesh $\mathrm{O}=\mathrm{m}^{-1} \sqrt{\mathrm{n}}$ if $\mathrm{n}$ is even.

b) $\operatorname{mesh} Q J=m^{-1} \sqrt{4 n-2}$.

PROOF.

a) Take in part al of theorem 2.6.2 the set $S$ equal to

$$
S=\{1,3, \ldots, n\} \quad \text { if } n \text { is odd }
$$

and

$$
\mathrm{s}=\{1,3, \ldots, \mathrm{n}-1\} \quad \text { if } \mathrm{n} \text { is even }
$$

b) Take in part $b$ of theorem 2.6.2 $S$ equal to $S=\{1,3,5, \ldots\}$ and $T$ equal to $T=\{2,4,6, \ldots$.$\} .$

Observe that mesh $\mathrm{K}=$ mesh $\mathrm{J}$ but that mesh $\mathrm{QK}$ is unequal to mesh QJ Clearly, the QJ triangulation yields long and skinny simplices and is therefore less suitable for use in fixed point algorithms on $S^{n}$ or $U^{n}$. Even the $Q$ triangulation is not very attractive as will appear in chapter 5 . In that chapter we propose a new triangulation which seems to be the most suitable triangulation of $\mathrm{s}^{\mathrm{n}}$ or $\mathrm{U}^{\mathrm{n}}$ for use in fixed point algorithms. Finally, we remark that the replacement step for the QAK triangulation is the same as for the $A K$ triangulation of $R^{n}$. Before discussing algorithms we give some applications of Brouwer's and Kakutani's fixed point theorems and we extend the latter to mappings on unbounded regions (cf. Merrill $[1971,1972])$. 
CHAPTER 3

EXTENSIONS AND APPLICATIONS OF FIXED POINT THEOREMS

3.1. INTRODUCTION.

To prove the existence of a solution to a problem in nonlinear mathematics, fixed point theorems are of extreme importance. Applications are numerous and include the existence of an equilibrium in economic models and game theory and (un)constrained optimization problems. However, in many applications the conditions of the fixed point theorem treated in the previous chapter are too strong. Therefore we will discuss a lemma due to Merrill that gives a weaker condition.

In section 2 we first treat the problem of the existence of an equilibriumstrategy vector in a noncooperative $\mathrm{N}$-person game. In section 3 Merrill's condition is stated and it is proved that this condition is sufficient for the existence of a fixed point of a mapping from $R^{n}$ to the set of nonempty, convex subsets of $R^{n}$. Finally, in the sections 4 and 5 some applications of Merrill's condition are discussed, viz. the constrained optimization problem and the nonlinear complementarity problem.

\subsection{NONCOOPERATIVE N-PERSON GAME.}

The noncooperative $\mathrm{N}$-person game can be characterized by $\mathrm{N}$ players, indexed by $j=1, \ldots, N$, and by $m_{j}+1$ pure strategies for player $j, j=1, \ldots, N$. If the set I denotes the product of index sets $I_{m_{j}+1}, j=1, \ldots, N$, then the vector $\underline{k}=\left(k_{1}, \ldots, k_{N}\right) \in I$ means that player ${ }^{j} j$ uses his $k_{j}$-th pure strategy. Therefore we call $\underline{\mathrm{k}} \in \mathrm{I}$ a strategy vector. Furthermore, let $a^{j}(\underline{k})$ be the loss to player $j$ if strategy $\underline{k}$ is played. Without loss of generality we can assume that each $a^{j}(\underline{k})$ is positive, $\underline{k} \in I, j \in I_{N}$. Moreover, let $s^{m_{j}}$ be the strategy space of player $j$, i.e. the $i-t h$ component of the vector $x^{j} \in s^{m_{j}}$ denotes the probability that player $j$ uses his $i$-th strategy. Finally, define $S$ by 


$$
S=\prod_{j=1}^{N} S^{m_{j}}
$$

Then $S$ represents the strategy space for the game. The $\Sigma_{j=1}^{N}\left(m_{j}+1\right)-$ dimensional vector $x=\left(x^{1}, \ldots, x^{N}\right)$ will denote an element of $S$ such that $x^{j} \in s^{m_{j}}, j \in I_{N}$. Now, the expected loss $p^{j}(x)$ to player $j$ if strategy $\mathrm{x} \in \mathrm{S}$ is played is given by

$$
p^{j}(x)=\sum_{\underline{k} \in I} a^{j}(\underline{k}) \underset{i=1}{N} x_{k_{i}^{i}}^{i} .
$$

The marginal loss to player $j$ if he plays his $h$-th pure strategy and the other players stick on strategy $x$, is given by

$$
m_{h}^{j}(x)=\sum_{\substack{k \in I \\ k_{j}=h}} a^{j}(\underline{k}) \prod_{\substack{i=1 \\ i \neq j}}^{N} x_{i}^{i}
$$

Clearly, $p^{j}(x)=\sum_{h=1}^{m_{j}+1} x_{h}^{j} m_{h}^{j}(x)$ for all $j \in I_{n}$ and $x \in S$.

DEFINITION 3.2.1. A point $\bar{x} \in S$ is an equilibrium-strategy vector of the game if for each player $j$

$$
m_{h}^{j}(\bar{x}) \geq p^{j}(\bar{x}) \quad h=1, \ldots, m_{j}+1
$$

We now prove that there exists indeed such an equilibrium vector (see Owen [1976]). Therefore we construct a continuous function f from the compact, convex set $S$ into itself such that a fixed point of $f$ is an equilibrium vector. Since $f$ has at least one fixed point, we obtain the desired result. Therefore, let for $j=1, \ldots, N$

$$
b_{h}^{j}(x)=\max \left(\underline{p}^{j}(x)-m_{h}^{j}(x), 0\right) \quad h=1, \ldots, m_{j}+1
$$

Next we define the continuous function $f$ from $S$ to $S$ by $f(x)=$ $\left(f^{1}(x), \ldots, f^{N}(x)\right)$, where $f^{j}(x)$ is the function from $S^{m_{j}}$ into itself defined by

$$
f_{h}^{j}(x)=\left(x_{h}^{j}+b_{h}^{j}(x)\right) /\left(1+\sum_{k=1}^{m_{j}+1} b_{k}^{j}(x)\right) \quad h=1, \ldots, m_{j}+1
$$


We will now show that if $\bar{x}$ is a fixed point of $f, \bar{x}$ is also an equilibrium-strategy vector. Since $\bar{x}$ is a fixed point of $f$, we have that

$$
\bar{x}_{h}^{j}=f_{h}^{j}(\bar{x})=\left(\bar{x}_{h}^{j}+b_{h}^{j}(\bar{x})\right) /\left(1+\sum_{k=1}^{m_{j}+1} b_{k}^{j}(\bar{x})\right) .
$$

Suppose now that for some player $l, b_{h}^{l}(\bar{x})$ is positive for some $h$.

Then the denominator above is larger than one and hence we must have that $b_{k}^{\ell}(\bar{x})>0$ for any $k$ such that $\bar{x}_{k}^{l}>0$. This implies that $p^{\ell}(\bar{x})>m_{k}^{\ell}(\bar{x})$ for all $k$ with $\bar{x}_{k}^{l}>0$, which contradicts the fact that $p^{j}(x)$ is a weighted average of the $m_{h}^{j}(x)$ with weights $x_{h}^{j}, j=1, \ldots, N$. Hence, $b_{h}^{l}(\bar{x}) \leq 0$ for all $h=1, \ldots, m_{j}+1$, which proves that $\bar{x}$ is an equilibrium-strategy vector. We remark that we will use another index-notation in chapter 6 .

To prove the existence of an equilibrium price-vector in economic models, a very similar function $f$ as above can be used, see e.g. Scarf [1973], Todd [1976a] and Van der Laan [1980]. However, by using the above defined functions $b$ and $f$, we loose important information about the quantities $\mathrm{p}$ and $\mathrm{m}$. This affects the efficiency of a fixed point algorithm. Therefore, we develop in chapter 6 a complementarity problem which is equivalent to the noncooperative $\mathrm{N}$-person game (cf. also Van der Laan and Talman [1978b]). The general nonlinear complementarity problem is discussed in section 5 .

\subsection{EXTENSIONS TO KAKUTANI'S THEOREM.}

Before giving some other applications we relax the conditions of Kakutani's theorem so that we get a fixed point theorem for mappings on $R^{n}$ or $R_{+}^{n}$. The first extension is due to Eaves [1970] (see also Todd [1976a]).

LEMMA 3.3.1. Let $\mathrm{C}$ be a compact and convex subset of $\mathrm{R}^{\mathrm{n}}$ and let $\phi$ be an upper semi-continuous mapping from $C$ to the set of nonempty, convex, compact subsets of $R^{n}$. Suppose that there exists some $c \epsilon$ int $C$ such that $c \in \phi(x)$ for all $x \in$ bd $C$. Then there is an $x^{*} \in C$ such that $x^{*} \epsilon \phi\left(x^{*}\right)$.

PROOF. Since $\phi$ is upper semi-continuous and $C$ is compact, the set $\phi(C)=\left\{y \in R^{n} \mid y \in \phi(x), x \in C\right\}$ is compact. Hence the convex hull of the set $C \cup \phi(C)$ is compact. Extend now $\phi$ to $\bar{\phi}$ in the same way as in the proof of theorem 2.4.3. Then, by the same theorem, $\bar{\phi}$ has a fixed point $x^{*}$. 
By repeating the second part of the proof of theorem 2.4.3, we get $\mathrm{x}^{*} \in \phi\left(\mathrm{x}^{*}\right)$.

The following extension is due to Merrill [1971,1972] and plays an important role in the convergence of many fixed point algorithms on $R^{n}$. In the sequel let $B(x, p)$ denote the set defined by

$$
B(x, \rho)=\left\{y \in R^{n}\left|\max _{i=1, \ldots, n}\right| y_{i}-x_{i} \mid \leq \rho\right\} \quad x \in R^{n}, \rho \geq 0 .
$$

LEMMA 3.3.2. (MERRILL'S CONDITION). Let $\phi$ be an u.s.c. mapping from $R^{n}$ to the set of nonempty, convex subsets of $R^{n}$. Suppose there exist $w \in R^{n}$, $\mu>0$ and $\rho>0$ such that for all $x \notin B(w, \mu), f(x) \in \phi(x)$ and $z \in B(x, \rho)$

$$
(f(x)-x)^{\top}(w-z)>0 .
$$

Then $\phi$ has a fixed point in $B(w, \mu)$.

PROOF. Let $\mathrm{C}$ be the compact, convex set $\mathrm{B}(w, 2 \mu)$. Define $\bar{\phi}$ from $\mathrm{C}$ to the set of nonempty, convex subsets of $R^{n}$ by

$$
\bar{\phi}(\mathrm{x})=\phi(\mathrm{x}) \quad \text { if } \mathrm{x} \in \text { int } \mathrm{C}
$$

and

$$
\bar{\phi}(x)=\operatorname{conv}(\phi(x) \cup\{w\}) \quad \text { if } x \in \text { bd } c .
$$

Clearly, $\bar{\phi}$ is an u.s.c. mapping such that $w \in \bar{\phi}(\mathrm{x})$ if $\mathrm{x} \in$ bd C. Therefore, by lemma $3.3 .1, \bar{\phi}$ has a fixed point $x^{*}$ in $C$. If $x^{*} \in B(w, \mu)$, then $x^{*} \epsilon$ int $C$ and hence $x^{*} \epsilon \phi\left(x^{*}\right)$. We now prove that $x^{*} \notin B(w, \mu)$ gives a contradiction. If $x^{*} \notin B(w, \mu)$, then $x^{*} \in \operatorname{conv}\left(\phi\left(x^{*}\right) \cup\{w\}\right)$, i.e. for some $\lambda, 0 \leq \lambda \leq 1$, and $f\left(x^{*}\right) \in \phi\left(x^{*}\right)$ we have that

$$
x^{*}=\lambda f\left(x^{*}\right)+(1-\lambda) w,
$$

and so

$$
\lambda\left(x^{*}-f\left(x^{*}\right)\right)+(1-\lambda)\left(x^{*}-w\right)=0 .
$$

From the condition of the lemma we know that $\left(x^{*}-f\left(x^{*}\right)\right)^{\top}\left(x^{*}-w\right)>0$. Moreover, $\left(x^{*}-w\right)^{\top}\left(x^{*}-w\right)>0$ so that the above equality gives a contradiction. 
Note that the theorem is also valid if the condition holds only for $\mathbf{x} \in$ bd $B(w, \mu)$, but for fixed point algorithms this condition is not strong enough (cf. Todd [1976a,page 63]). The next two sections are based on the work of Merrill [1971,1972].

\subsection{CONSTRAINED OPTIMIZATION.}

In this section we consider the problem

$$
\min \left\{f(x) \mid g_{i}(x) \leq 0, i=1, \ldots, m\right\}
$$

where $f$ and $g_{i}$ are functions from $R^{n}$ to $R$. All functions are assumed to be convex (and finite). Furthermore, let $\delta \mathrm{h}$ be the set of all subgradients of a function $h: R^{n} \rightarrow R$, i.e.

$$
\delta h(x)=\left\{y \in R^{n} \mid h(z) \geq h(x)+y^{\top}(z-x) \text { for all } z \text { in } R^{n}\right\}
$$

Note that for a convex function $h, 0 \epsilon \delta h\left(x^{*}\right)$ if and only if $x^{*}$ minimizes $h(x), x \in R^{n}$. Moreover, if $h$ is convex, then $\delta h$ is an u.s.c. mapping from $R^{n}$ to the set of nonempty, convex subsets of $R^{n} \quad$ (Rockafellar [1970, theorem 2.4.5.1]). Next, define the function $s$ from $R^{n}$ to $R$ by

$$
s(x)=\max _{1 \leq i \leq m} g_{i}(x) .
$$

If $I(x)=\left\{i \mid s(x)=g_{i}(x)\right\}$, then $\delta s(x)=\operatorname{conv}\left(\underset{i \in I(x)}{u} \delta g_{i}(x)\right)$.

Since $s$ and $f$ are both convex, we have that $\delta s$ and $\delta f$ are u.s.c. mappings from $R^{n}$ to the set of nonempty, convex subsets of $R^{n}$. Next, define the mapping $\phi$ by

$$
\begin{array}{rlrl}
\phi(x) & =\{x\}-\delta f(x) & & s(x)<0 \\
& =\{x\}-\operatorname{conv}(\delta f(x) \cup \delta s(x)) & & s(x)=0 \\
& =\{x\}-\delta s(x) & s(x)>0,
\end{array}
$$


where for any two sets $A, B \subset R^{n}$ the set $A-B$ is defined by

$$
A-B=\{x-y \mid x \in A, y \in B\} .
$$

It is easy to see that $\phi$ is an u.s.c. mapping from $R^{n}$ to the set of nonempty, convex subsets of $\mathrm{R}^{\mathrm{n}}$.

LEMMA 3.4.1. If there exists a point $x \in \mathrm{R}^{\mathrm{n}}$ with $\mathrm{s}(\mathrm{x})<0$, then the set of optimal solutions to the problem $(P)$ is the set of fixed points of $\phi$.

PROOF. Let $x^{*}$ be an optimal solution. Then $x^{*}$ minimizes $f+\lambda^{*} s$ for some $\lambda^{*} \geq 0$ such that $\lambda^{*} s\left(x^{*}\right)=0$ (see Rockafellar [1970, corollary 28.2.1]) if $s(x)<0$ for some $x$. So, since $0 \in \delta f\left(x^{*}\right)+\lambda^{*} \delta s\left(x^{*}\right)$, there exist $a \in \delta f\left(x^{*}\right)$ and $b \in \delta s\left(x^{*}\right)$ such that

$$
\left(1+\lambda^{*}\right)^{-1} a+\lambda^{*}\left(1+\lambda^{*}\right)^{-1} b=0
$$

Hence, $\lambda^{*}=0$ implies $0 \in \delta f\left(x^{*}\right)$ and $x^{*} \in \phi\left(x^{*}\right)$. If $\lambda^{*}>0$, then $0 \in \operatorname{conv}\left(\delta f\left(x^{*}\right) \cup \delta s\left(x^{*}\right)\right)$ and further $s\left(x^{*}\right)=0$. Consequently, again $\mathrm{x}^{*} \in \phi\left(\mathrm{x}^{*}\right)$.

Conversely, let $x^{*}$ be a fixed point of $\phi$. If $s\left(x^{*}\right)>0$, then $0 \in \delta s\left(x^{*}\right)$ and so $x^{*}$ minimizes $s(x), x \in R^{n}$, and therefore $s(x)>0$ so that the condition of the lemma does not apply. If $s\left(x^{*}\right)<0$, then $0 \in \delta f\left(x^{*}\right)$ and so $x^{*}$ solves the problem (P).

Finally assume that $s\left(x^{*}\right)=0$. Then $0 \in \operatorname{conv}\left(\delta f\left(x^{*}\right) \cup \delta s\left(x^{*}\right)\right)$ and hence there exist $a \in \delta f\left(x^{*}\right)$ and $b \in \delta s\left(x^{*}\right)$ such that for some $\lambda^{*}, 0 \leq \lambda^{*} \leq 1$,

$$
\lambda^{*} a+\left(1-\lambda^{*}\right) b=0
$$

If $\lambda^{*}=0$, then $0 \in \delta s\left(x^{*}\right)$ and so $x^{*}$ minimizes s, i.e. $s(x) \geq 0$ for all $x$. However, if $\lambda^{*}$ is positive, then $a+\lambda^{*-1}\left(1-\lambda^{*}\right) b=0$ and $x^{*}$ minimizes $f+\lambda^{*-1}\left(1-\lambda^{*}\right) \mathrm{s}$. Again, by Rockafeller [1970, corollary 28.2.1], $\mathrm{x}^{*}$ solves the problem (P).

In the next theorem we state that Merrill's condition is satisfied if for some $\alpha$ the level set $\{y \mid s(y) \leq \alpha\}$ is nonempty and bounded. 
THEOREM 3.4.2. If for some $\alpha$ the set $\{y \mid s(y) \leq \alpha\}$ is nonempty and bounded, then for any $w \in R^{n}$ and $\rho>0$ there exists a $\mu>0$ such that Merrill's condition is satisfied for $\phi$.

PROOF. Since $s$ is convex and $\{y \mid s(y) \leq \alpha\}$ is nonempty and bounded for some $\alpha$, the set $\{y \mid s(y) \leq \gamma\}$ is also bounded for each $\gamma$. For given $w \in R^{n}$ and $\rho>0$ let

$$
\beta=\max [0, \max \{s(x) \mid x \in B(w, p)\}] \text {. }
$$

Thus $\{y \mid s(y) \leq \beta\}$ is bounded. Choose $\mu$ such that the latter set is contained in $B(w, \mu)$. Note that $\phi(x)=\{x\}-\delta s(x)$ for $x \notin\{y \mid s(y) \leq \beta\}$ since $\beta \geq 0$. Choose for any $x \notin B(w, \mu)$ some $g(x) \in \phi(x)$ and $z \in B(x, p)$. Then we have $x-g(x) \in \delta s(x)$ and so

$$
(g(x)-x)^{\top}(w-z)=(g(x)-x)^{\top}((w-z+x)-x) \geq s(x)-s(w-z+x) .
$$

Since $w-z+x \in B(w, p)$ we have that $s(w-z+x) \leq \beta$. Moreover, $s(x)>\beta$. Consequently

$$
(g(x)-x)^{\top}(w-z)>0
$$

which proves the theorem.

In the same way the unconstrained optimization problem can be treated (see e.g. Merrill [1971,1972], Lüthi [1976], Todd [1976a] and Van der Laan [1980]).

3.5. NONLINEAR COMPLEMENTARITY PROBLEM.

Let $f$ be continuous from $R_{+}^{n}$ to $R^{n}$. The nonlinear complementarity problem is to find a point $x^{*} \in R_{+}^{n}$ such that

$$
f\left(x^{*}\right) \geq 0 \text { and } \sum_{i=1}^{n} x_{i}^{*} f_{i}\left(x^{*}\right)=0 \text {. }
$$

This problem, to be denoted by (NLCP), was first studied by Cottle [1966]. The following theorem due to Moré [1974] gives a sufficient condition for 
the existence of a solution. The proof makes use of Brouwer's fixed point theorem.

THEOREM 3.5.1. Assume there exist $w \in R_{+}^{n}$ and some $\rho>\max _{i} w_{i}$ such that for all $x \in R_{+}^{n}$ with $\max _{i} x_{i} \geq \rho$ holds

$$
\max _{i=1, \ldots, n}\left(x_{i}-w_{i}\right) f_{i}(x)>0
$$

Then the (NLCP) has a solution $x^{*}$ in the set $B_{\rho}=\left\{x \in R_{+}^{n} \mid \max _{i} x_{i} \leq \rho\right\}$.

PROOF. Let $h: R_{+}^{n} \rightarrow R^{n}$ be the function defined by (componentswise)

$$
h(x)=\max \{\underline{0}, x-f(x)\} .
$$

Clearly, $x^{*}$ solves the problem if and only if $h\left(x^{*}\right)=x^{*}$. So, we now prove that $h$ has a fixed point in $B_{\rho}$. Since $h$ is continuous, $B_{\rho} u h\left(B_{\rho}\right)$ is compact. Let $\tau$ be such that $B_{\rho} \cup h\left(B_{\rho}\right)$ is a subset of $B_{\tau}$. Define the function $r: B_{\tau} \rightarrow B_{\rho}$ by

where

$$
\begin{array}{rlrl}
r(x) & =x & x \in B_{\rho} \\
& =\lambda(x) x+(1-\lambda(x)) w & x \notin B_{\rho},
\end{array}
$$

$$
\lambda(x)=\min \left\{\left(\rho-w_{i}\right) /\left(x_{i}-w_{i}\right) \mid x_{i}>\rho\right\} .
$$

Clearly, $r(\mathrm{x}) \in \mathrm{B}_{\rho}$ if $\mathrm{x} \in \mathrm{B}_{\tau}$, and both functions $\lambda$ and $x$ are continuous. Define now the continuous function $g$ from the compact, convex set $B_{\tau}$ into itself by $g(x)=h(r(x))$. By Brouwer's theorem, $g$ has a fixed point $x^{*}$. Of course $h\left(x^{*}\right)=x^{*}$ if $x^{*} \in B_{\rho}$. Suppose $x^{*} \notin B_{\rho}$. By the definition of $\lambda(x), \max _{j} r_{j}\left(x^{*}\right)=\rho$. Let $i$ be an index such that

$$
\left(r_{i}\left(x^{*}\right)-w_{i}\right) f_{i}\left(r\left(x^{*}\right)\right)>0 .
$$

If $r_{i}\left(x^{*}\right)>w_{i}$, we have

$$
x_{i}^{*} \geq r_{i}\left(x^{*}\right)>w_{i} \geq 0 \text { and } x_{i}^{*} \geq r_{i}\left(x^{*}\right)>r_{i}\left(x^{*}\right)-f_{i}\left(r\left(x^{*}\right)\right) \text {. }
$$


If $r_{i}\left(x^{*}\right)<w_{i}$, we have

$$
0 \leq x_{i}^{*} \leq r_{i}\left(x^{*}\right)<r_{i}\left(x^{*}\right)-f_{i}\left(r\left(x^{*}\right)\right) .
$$

Hence, in both cases

$$
x_{i}^{*} \neq \max \left\{0, r_{i}\left(x^{*}\right)-f_{i}\left(r\left(x^{*}\right)\right)\right\}=g_{i}\left(x^{*}\right),
$$

which contradicts the fact that $\mathrm{x}^{*}$ is a fixed point of $\mathrm{g}$.

Merrill [1971,1972] represented the nonlinear complementarity problem directly as a fixed point problem and gave a sufficient condition for the condition of lemma 3.3.2. Therefore, let us define the convex function $g: \mathrm{R}^{\mathrm{n}} \rightarrow \mathrm{R}^{\mathrm{n}}$ by

$$
g(x)=-\min _{i} x_{i}
$$

and the mapping $\phi$ by

$$
\begin{aligned}
\phi(x) & =\{x-f(x)\} & & x \in \text { int } R_{+}^{n} \\
& =\{x\}-\operatorname{conv}(\{f(x)\} \cup \delta g(x)) & & x \in b d R_{+}^{n} \\
& =\{x\}-\delta g(x) & & x \notin R_{+}^{n} .
\end{aligned}
$$

Since $f$ and $g$ are both convex, $\phi$ is an u.s.c. mapping from $R^{n}$ to the set of nonempty, convex subsets of $\mathrm{R}^{\mathrm{n}}$.

LEMMA 3.5.2. The point $\mathrm{x}^{*}$ solves the (NLCP) iff $\mathrm{x}^{*}$ is a fixed point of $\phi$.

PROOF. Let $\mathrm{x}^{*} \in \phi\left(\mathrm{x}^{*}\right)$. If $\mathrm{x}^{*} \notin \mathrm{R}_{+^{\prime}}^{\mathrm{n}}$, then $0 \in \delta g\left(\mathrm{x}^{*}\right)$ and $\mathrm{x}^{*} \operatorname{minimizes} \mathrm{g}(\mathrm{x})$, $x \in R^{n}$. This contradicts the fact that $g(x)>0$ if $x \notin R_{+}^{n}$ and $g(x) \leq 0$ for $x \in R_{+}^{n}$. Hence, $x^{*} \in R_{+}^{n}$. of course, $x^{*}$ solves the (NLCP) if $x^{*} \in$ int $R_{+}^{n}$. So, let $\mathrm{x}^{*} \in \mathrm{bdR}_{+}^{\mathrm{n}}$. Then it is easily seen that

$$
\delta g\left(x^{*}\right)=-\operatorname{conv}\left(u\left\{e(i) \mid i \in I\left(x^{*}\right)\right\}\right),
$$

where $I(y)=\left\{i \mid y_{i}=0\right\}, y \in R^{n}$. Consequently, $f_{i}\left(x^{*}\right)=0$ for all indices $i \notin I\left(x^{*}\right)$. Moreover, $f_{i}\left(x^{*}\right) \geq 0$ for $i \in I\left(x^{*}\right)$. Together this proves that 
$x^{*}$ solves the (NLCP).

Conversely, let $x^{*}$ be a solution of the nonlinear complementarity problem. If $f\left(x^{*}\right)=0$ we must have that $x^{*} \in \phi\left(x^{*}\right)$. Note that $f\left(x^{*}\right)=0$ if $I\left(x^{*}\right)=\varnothing$. Suppose now that $f\left(x^{*}\right) \geq 0$ and $f\left(x^{*}\right) \neq 0$ so that $I\left(x^{*}\right) \neq \varnothing$. Then $\alpha=\sum_{i} f_{i}\left(x^{*}\right)>0$. Consequently,

$$
f\left(x^{*}\right)=\sum_{i=1}^{n} f_{i}\left(x^{*}\right) e(i) \in \alpha \operatorname{conv}\left(u\left\{e(i) \mid i \in I\left(x^{*}\right)\right\}\right) .
$$

Hence, $-f\left(x^{*}\right) \in \alpha \delta g\left(x^{*}\right)$. Note that

$$
x^{*}=(1+\alpha)^{-1} \alpha\left\{x^{*}+\alpha^{-1} f\left(x^{*}\right)\right\}+(1+\alpha)^{-1}\left\{x^{*}-f\left(x^{*}\right)\right\}
$$

Since $x^{*}+\alpha^{-1} f\left(x^{*}\right) \in\left\{x^{*}\right\}-\delta g\left(x^{*}\right)$ and $x^{*}-f\left(x^{*}\right) \in\left\{x^{*}\right\}-\left\{f\left(x^{*}\right)\right\}$, we obtain that $x^{*} \in \phi\left(x^{*}\right)$ which proves the lemma.

In the next theorem we give a sufficient condition for the existence of a fixed point of the mapping $\phi$, where ||$y||=\max _{i}\left|y_{i}\right|$.

THEOREM 3.5.3. Suppose there exist $\alpha, \beta>0$ such that

$$
\sum_{i=1}^{n} x_{i} f_{i}(x)>\alpha\|x\| \cdot\|f(x)\| \text { if } x \in R_{+}^{n} \backslash B(0, \beta)
$$

Then for any $\rho>0$ there exist $w \in \mathrm{R}^{\mathrm{n}}$ and $\mu>0$ such that Merrill's condition is satisfied for the mapping $\phi$.

PROOF. Let $w$ be such that $w_{i}>\rho$ for all $i$, and let

$$
\mu>\max \left\{\beta+\|w\|, \alpha^{-1}(\rho+\|w\|)\right\} .
$$

Furthermore, let $x \notin B(w, \mu), f \in \phi(x)$ and $z \in B(x, p)$. If $g(x)>0$ then $x-f \in \delta g(x)$. Thus, as in the proof of theorem 3.4 .3

$$
(f-x)^{\top}(w-z) \geq g(x)-g(w+x-z),
$$

which is positive since $g(y)<0$ for all $y \in B(w, \rho)$. So, Merrill's condition is satisfied. If $g(x)<0$, then $x \in R_{+}^{n}$ and $x \notin B(0, \beta)$. Hence, $f=x-f(x)$ which implies 
36

$$
\begin{aligned}
(f-x)^{\top}(w-z) & =-f(x)^{\top}(w-z)=f(x)^{\top} x+f(x)^{\top}(-w-x+z) \\
& >\alpha\|x\| \cdot\|f(x)\|-\|f(x)\| \cdot\|z-x-w\| \\
& \geq\|f(x)\|(\alpha\|x\|-\|w\|-\rho)>0,
\end{aligned}
$$

since $f(x)$ cannot be 0 .

Finally, if $g(x)=0$ we can combine both cases above to prove again that Merrill's condition is satisfied, i.e. that $(f-x)^{\top}(w-z)$ is positive. 
CHAPTER 4

ALGORITHMS TO FIND COMPLETELY LABELLED SIMPLICES

\subsection{INTTRODUCTION.}

In this chapter algorithms to approximate a fixed point of a continuous function or u.s.c. mapping from an m-dimensional convex subset of $\mathrm{R}^{\mathrm{n}}$ into itself are discussed. Except for scarf's original method all these algorithms take a sequence of triangulations of the set such that the mesh of the triangulation tends to zero. Then the vertices of the triangulations are labelled with one of the integers $\{1, \ldots, m+1\}$ (or with an $(m+1)$-vector) in such a way that a completely labelled m-simplex yields an approximate fixed point. A fixed point algorithm now tries to find a sequence of completely labelled simplices, one for each triangulation. Then, if the mesh goes to zero, the accuracy of the approximation increases. Moreover, in the limit at least one subsequence of completely labelled simplices converges to a fixed point.

To generate an approximate fixed point on the unit simplex $\mathrm{s}^{\mathrm{n}}$ Scarf introduced in 1967 an algorithm based on the concept of primitive sets rather than based on a triangulation of $s^{n}$. Using Scarf's basic ideas Kuhn developed in 1968 and 1969 two closely related algorithms on $s^{\text {n }}$ based on the standard triangulation of $s^{\mathrm{n}}$ and only for integer labelling. In 1971 Eaves presented an algorithm for vector labelling to compute fixed points of an u.s.c. mapping on a convex compact subset of $R^{n}$. However, all these algorithms suffer from computational inefficiency since they must start on the boundary of the set or outside the region of interest. Consequently, in these algorithms an obtained approximation cannot be used as the starting point in the next application of the algorithm with a smaller grid size. A large number of algorithms avoiding this disadvantage was developed during the seventies. One method is due to Merrill [1971,1972] and was indenendently found by Kuhn and Mackinnon [1975], Lüthi [1975,1976] and Fisher, Gould and Tolle 51977$]$. This 
algorithm can start anywhere and was developed for problems on $S^{n}$ and $R^{n}$ both for integer and vector labelling. Thus, for a decreasing sequence of grid sizes the algorithm can be applied such that in each step information about the approximation in the previous step can be utilized to determine the new grid size and the new starting point.

Another method taking advantage of available information is the homotopyalgorithm introduced for integer and vector labelling by Eaves [1972] on $S^{n}$ and by Eaves and Saigal [1972] on $R^{n}$. In their method the grid size is automatically refined during the algorithm. However, both the restart method of Merrill and the homotopy-algorithm of Eaves and Saigal operate in an $(n+1)$-dimensional subset and generate a path of full-dimensional adjacent simplices of a triangulation of this set. Furthermore, in Merrill's algorithm an extra level of artificially labelled points is needed whereas the factor of incrementation for the homotopy-algorithm is at most two.

Van der Laan and Talman [1979a,1979b] developed a restart algorithm on $S^{n}$ and $\mathrm{R}^{\mathrm{n}}$ which avoids the introduction of an extra dimension. Moreover, their algorithm starts with a single point and generates then a path of adjacent simplices of variable dimension until a completely labelled n-simplex is generated (see also Van der Laan [1980]). Further, Van der Laan and Talman [1980b] considerably improved the homotony-algorithms. Using ideas of their restart method, they introduced a homotopy-algorithm on $S^{n}$ and $R^{n}$ in which the factor of incrementation can be of any size.

This chapter is organized as follows. In section 2 the standard labelling rules on $S^{n}$ and $R^{n}$ are presented both for integer and vector labelling. Also theorems about the accuracy of an approximate fixed point are given. In section 3 one of Kuhn's algorithms and Eaves' first algorithm are discussed. Section 4 gives a short description of Merrill's algorithm. The basic variable dimension algorithm of Van der Laan and Talman is discussed in section 5. Also in section 5 attention is paid. to the continuous - deformation algorithms.

\subsection{LABELLING AND ACCURACY.}

To prove that a continuous function from the unit simplex $s^{\mathrm{n}}$ into itself has at least one fixed point, a sequence of triangulations $G_{k}$ with mesh $G_{k} \rightarrow 0$ as $k \rightarrow \infty$ was taken and each vertex was labelled by an integer $\ell(\cdot)$. In this section we redefine this labelling rule. 
DEFINITION 4.2.1. (STANDARD INTEGER LABELLING ON $\mathrm{s}^{\mathrm{n}}$ ). A point $\mathrm{x} \in \mathrm{s}^{\mathrm{n}}$ receives the label $\ell(x) \cdot \epsilon I_{n+1}$ where $\ell(x)=i$ if

$$
i=\min \left\{j \mid f_{j}(x)-x_{j}=\min _{h \in I_{n+1}}\left(f_{h}(x)-x_{h}\right)\right\}
$$

Clearly $\ell(x) \neq i$ if $x_{i}=0$, except when $f(x)=x$ and $x_{1}=0$. In this case we do not assign the label 1 to $x$ but the first index $i$ such that $x_{i}>0$. So, since the labelling rule is proper, we have, by Sperner's lemma, that each triangulation $G$ has at least one completely labelled simplex. We now show how close an arbitrary point $x$ in a completely labelled simplex of a given triangulation $G$ is to its image $f(x)$.

LEMMA 4.2.2. Let $\varepsilon, \delta>0$ be such that mesh $G=\delta$ and

$$
\max _{j \in I_{n+1}}\left|f_{j}(x)-f_{j}(y)\right|<\varepsilon \quad \text { for all } x, y \in \sigma, \sigma \in G .
$$

Then for any $\mathrm{x}^{*}$ in a completely labelled simplex $\sigma$ in $\mathrm{G}$

$$
\max _{j \in I_{n+1}}\left|f_{j}\left(x^{*}\right)-x_{j}^{*}\right|<n(\varepsilon+\delta) .
$$

PROOF. Let $y^{i}$ be the vertex of $\sigma$ with $\ell\left(y^{i}\right)=i, i=1, \ldots, n+1$. clearly, for $i \in I_{n+1}$

$$
f_{i}\left(x^{*}\right)-x_{i}^{*}=\left(f_{i}\left(x^{*}\right)-f_{i}\left(y^{i}\right)\right)+\left(f_{i}\left(y^{i}\right)-y_{i}^{i}\right)+\left(y_{i}^{i}-x_{i}^{*}\right) .
$$

Since $\ell\left(y^{i}\right)=i$, the second term on the right side of this relation is nonpositive. Moreover, since $\mathrm{x}^{*}$ and $\mathrm{y}^{i} \in \sigma$,

$$
y_{i}^{i}-x_{i}^{*} \leq \delta \text { and } f_{i}\left(x^{*}\right)-f_{i}\left(y^{i}\right)<\varepsilon, i=1, \ldots, n+1 .
$$

Combining these facts together, we obtain

$$
f_{i}\left(x^{*}\right)-x_{i}^{*}<\varepsilon+\delta .
$$


On the other hand, since $\sum_{j=1}^{n+1}\left(f_{j}\left(x^{*}\right)-x_{j}^{*}\right)=0$, we have for all $i$

$$
f_{i}\left(x^{*}\right)-x_{i}^{*}=-\sum_{j \neq i}\left(f_{j}\left(x^{*}\right)-x_{j}^{*}\right)>-n(\varepsilon+\delta),
$$

and so with the previous inequality we get the desired result.

The lemma guarantees that a completely labelled simplex of a triangulation of $S^{n}$ yields an approximation of a fixed point if the standard labelling rule is used, where $\max _{i}\left|f_{i}\left(x^{*}\right)-x_{i}^{*}\right|$ can be made as small as required by taking the mesh $\delta$ small enough. Note that assigning an integer label $i$ to a point $x$ means that almost all information about the vector $f(x)-x$ is lost. Only the fact that the $i$-th component of $f(x)-x$ is smaller than the other components is used. To overcome this loss of information we can assign a whole vector to the point $x$. Let e be the $(n+1)$-vector $(1, \ldots, 1)^{\top}$. DEFINITION 4.2.3. (STANDARD VECTOR LABELLING ON $\mathrm{S}^{\mathrm{n}}$ ). A point $\mathrm{x} \in \mathrm{S}^{\mathrm{n}}$ receives the $(n+1)$-vector $\ell(x)$ where

$$
\ell(x)=-f(x)+x+e .
$$

Analogously to the definition of a completely labelled simplex for integer labelling we have the following definition for vector labelling.

DEFINITION 4.2.4. A simplex $\sigma\left(y^{1}, \ldots, y^{n+1}\right)$ of a triangulation $G$ of $s^{n}$ is completely labelled if the system of $n+1$ linear equations

$$
\sum_{i=1}^{n+1} \lambda_{i} \ell\left(y^{i}\right)=e
$$

has a nonnegative solution $\lambda^{*}=\left(\lambda_{1}^{*}, \ldots, \lambda_{n+1}^{*}\right)$.

The following lemma states that a completely labelled simplex in G yields an approximate fixed point.

LEMMA 4.2.5. The piecewise linear approximation $\overline{\mathrm{f}}$ to the continuous function $f$ with respect to $G$ has a fixed point in the simplex $\sigma$ if and only if $\sigma$ is completely labelled. If $\lambda^{*}$ is the solution of the system of equations of a completely labelled simplex $\sigma\left(y^{1}, \ldots, y^{n+1}\right)$, then the point 


$$
x^{*}=\sum_{i=1}^{n+1} \lambda_{i}^{*} y^{i}
$$

is a fixed point of $\overline{\mathrm{f}}$ in $\sigma$.

PROOF. Let $\sigma\left(y^{1}, \ldots, y^{n+1}\right)$ be a completely labelled simplex with solution $\lambda^{*}$. Since $\sum_{i=1}^{n+1}\left(f_{i}(x)-x_{i}\right)=0$ for $x \in s^{n}$, we find by adding up all equations of the system that

$$
\sum_{i=1}^{n+1} \lambda_{i}^{*}=1
$$

Hence $x^{*}=\Sigma_{i=1}^{n+1} \lambda_{i}^{*} y^{i}$ lies in $\sigma\left(y^{1}, \ldots, y^{n+1} L\right.$ and

$$
-\bar{f}\left(x^{*}\right)+x^{*}=\sum_{i=1}^{n+1} \lambda_{i}^{*}\left(-f\left(y^{i}\right)+y^{i}\right)=\sum_{i=1}^{n+1} \lambda_{i}^{*} l\left(y^{i}\right)-e=0,
$$

which proves that $x^{*}$ is a fixed point of $\bar{f}$ in $\sigma$.

Conversely, if $x$ is a point in $\sigma\left(y^{1}, \ldots, y^{n+1}\right)$, there is a unique nonnegative vector $\lambda=\left(\lambda_{1}, \ldots, \lambda_{n}\right)$ with $\Sigma_{i=1}^{n+1} \lambda_{i}=1$ such that $x=\sum_{i=1}^{n+1} \lambda_{i} y^{i}$. Hence

$$
-\bar{f}(x)+x=\sum_{i=1}^{n+1} \lambda_{i}\left(-f\left(y^{i}\right)+y^{i}+e\right)-e=\sum_{i=1}^{n+1} \lambda_{i} \ell\left(y^{i}\right)-e .
$$

If $x^{*}$ is a fixed point of $\bar{f}$ in $\sigma$, then $\bar{f}\left(x^{*}\right)-x^{*}=0$, which implies that

$$
\sum_{i=1}^{n+1} \lambda_{i}^{*} l\left(y^{i}\right)=e,
$$

where the $\lambda_{i}^{*}$ 's are the unique nonnegative numbers such that $x^{*}=\sum_{i=1}^{n+1} \lambda_{i}^{*} y^{i}$. Therefore, by definition, $\sigma\left(y^{1}, \ldots, y^{n+1}\right)$ is completely labelled.

In the next theorem the accuracy of $x^{*}$ as approximate fixed point is given.

THEOREM 4.2.6. Let $\varepsilon, \delta>0$ be such that mesh $G=\delta$ and

$$
\max _{j \in I_{n+1}}\left|f_{j}(x)-f_{j}(y)\right|<\varepsilon \quad \text { for all } x, y \in \sigma, \sigma \in G \text {. }
$$

Then, if $\mathrm{x}^{\star}$ is defined as in lemma 4.2.5, 


$$
\max _{j \in I_{n+1}}\left|f_{j}\left(x^{*}\right)-x_{j}^{*}\right|<\varepsilon .
$$

PROOF. From the proof of theorem 4.2 .5 we have that

$$
x^{*}=\sum_{i=1}^{n+1} \lambda_{i}^{*} y^{i}=\sum_{i=1}^{n+1} \lambda_{i}^{*} f\left(y^{i}\right) \text { with } \sum_{i=1}^{n+1} \lambda_{i}^{*}=1 \text { and } \lambda_{i}^{*} \geq 0 \text { for all } i
$$

Consequently, since $\max _{j}\left|f_{j}\left(y^{i}\right)-f_{j}\left(x^{*}\right)\right|<\varepsilon$ for all $i$,

$$
\max _{j \in I_{n+1}}\left|f_{j}\left(x^{*}\right)-x_{j}^{*}\right|=\max _{j \in I}\left|\sum_{n+1}^{n+1} \lambda_{i}^{*}\left(f_{j}\left(x^{*}\right)-f_{j}\left(y^{i}\right)\right)\right|<\sum_{i=1}^{n+1} \lambda_{i}^{*} \varepsilon=\varepsilon,
$$

which proves the theorem.

This theorem states that using vector labelling a much better approximation can be obtained than for integer labelling, in particular when the dimension of the problem is large.

To approximate a fixed point (if any) of a continuous function $f$ from $R^{n}$ to $\mathrm{R}^{\mathrm{n}}$, we take again a sequence of triangulations of $\mathrm{R}^{\mathrm{n}}$ with mesh tending to zero. Now, a point in $\mathrm{R}^{\mathrm{n}}$ is in case of. integer labelling again labelled by an integer of the set $I_{n+1}$.

DEFINITION 4.2.7. (STANDARD INTEGER LABELLING ON $\mathrm{R}^{\mathrm{n}}$ ). For any $\mathrm{x} \in \mathrm{R}^{\mathrm{n}}$ let

$$
i=\min \left\{j \mid f_{j}(x)-x_{j}=\max _{h \in I_{n}}\left(f_{h}(x)-x_{h}\right)\right\} .
$$

Then $\mathrm{x}$ receives the label $\ell(\mathrm{x})$ where

and

$$
\ell(x)=i \quad \text { if } f_{i}(x)-x_{i} \geq 0
$$

$$
\ell(x)=n+1 \quad \text { if } f_{i}(x)-x_{i}<0
$$

Note that the number of labels is again equal to the number of vertices of a full-dimensional simplex so that a completely labelled n-simplex is well defined. The next theorem says that such a simplex yields an approximate fixed point. 
LEMMA 4.2.8. Let $\varepsilon, \delta>0$ be such that $\max _{j}\left|x_{j}-y_{j}\right| \leq \delta$ and $\max _{j}\left|f_{j}(x)-f_{j}(y)\right|<\varepsilon$ for all $x$ and $y$ in a completely labelled simplex $\sigma$. Then for any $x^{\star} \in \sigma$

$$
\max _{j \in I_{n}}\left|f_{j}\left(x^{*}\right)-x_{j}^{*}\right|<\varepsilon+\delta .
$$

PROOF. Let again $y^{i}$ be the vertex of $\sigma$ having label $i, i=1, \ldots, n+1$. Then, following the arguments in the proof of lemma 4.2.2, we get for all i. $\epsilon I_{n}$

$$
f_{i}\left(x^{*}\right)-x_{i}^{*}=\left(f_{i}\left(x^{*}\right)-f_{i}\left(y^{i}\right)\right)+\left(f_{i}\left(y^{i}\right)-v_{i}^{i}\right)+\left(y_{i}^{i}-x_{i}^{*}\right)>-(\varepsilon+\delta)
$$

and

$$
f_{i}\left(x^{*}\right)-x_{i}^{*}=\left(f_{i}\left(x^{*}\right)-f_{i}\left(y^{n+1}\right)\right)+\left(f_{i}\left(y^{n+1}\right)-y_{i}^{n+1}\right)+\left(y_{i}^{n+1}-x_{i}^{*}\right)<\varepsilon+\delta .
$$

Together these inequalities give the result.

$\square$

Finally, we consider vector labelling on $\mathrm{R}^{\mathrm{n}}$.

DEFINITION 4.2.9. (STANDARD VECTOR LABELIING ON $R^{n}$ ). A point $x \in R^{n}$ receives the $(n+1)$-vector $l(x)$ where

and

$$
l_{i}(x)=f_{i}(x)-x_{i}+1 \quad i=1, \ldots, n
$$

$$
\ell_{n+1}(x)=1
$$

DEFINITION 4.2.10. An n-simplex $\sigma\left(\mathrm{y}^{1}, \ldots, \mathrm{y}^{\mathrm{n}+1}\right)$ of a triangulation $\mathrm{G}$ of $\mathrm{R}^{\mathrm{n}}$ is completely labelled if the system of $n+1$ linear equations

$$
\sum_{i=1}^{n+1} \lambda_{i} \ell\left(y^{i}\right)=e
$$

has a nonnegative solution $\lambda^{*}=\left(\lambda_{1}^{*}, \ldots, \lambda_{n+1}^{*}\right)$.

Observe that the last equation of the system is equal to $\sum_{i=1}^{n+1} \lambda_{i}=1$. Then, following the proof of theorem 4.2 .6 , it can easily be seen that a completely labelled simplex yields a good approximate fixed point. 
COROLLARY 4.2.11. Let $\varepsilon, \delta>0$ be such that $\max _{j}\left|x_{j}-y_{j}\right| \leq \delta$ and $\max _{j}\left|f_{j}(x)-f_{j}(y)\right|<\varepsilon$ for all $x$ and $y$ in a completely labelled simplex $\sigma\left(y^{1}, \ldots, y^{n+1}\right)$ in $G$. Then, with $x^{*}=\sum_{i=1}^{n+1} \lambda_{i}^{*} y^{i}$, we have that

$$
\max _{j \in I_{n}}\left|f_{j}\left(x^{*}\right)-x_{j}^{*}\right|<\varepsilon .
$$

Moreover, $\mathrm{x}^{*}$ is a fixed point of the piecewise linear approximation $\overline{\mathrm{f}}$ to $f$ with respect to $G$.

The results of the theorems 4.2.5, 4.2.6 and 4.2.11 can be generalized to an upper semi-continuous mapping $\phi$ on $S^{n}$ and $R^{n}$ respectively (see Todd [1976a]). Note that integer labelling is not suitable for u.s.c. mappings (see Todd [1976a, page 58]). In case of vector labelling we choose an arbitrary element $f(x) \in \phi(x)$. However, it is important that if in the course of a fixed point algorithm $f(x)$ must be chosen for a second time, it will be the same choice as made before. In the next sections we discuss algorithms which generate completely labelled simplices of a given triangulation of $\mathrm{s}^{\mathrm{n}}$ or $\mathrm{R}^{\mathrm{n}}$.

\subsection{KUHN'S AND EAVES' FIRST ALGORTTHMS.}

Since Scarf's original algorithm on $S^{\mathrm{n}}$ is based on primitive sets (see Scarf $[1967,1973]$ ) we pay no attention to this method. Already in 1968 and 1969 Kuhn introduced two algorithms for integer labelling on the unit simplex, both making a search for a completely labelled simplex of the standard triangulation of $s^{n}$. The main ideas are however based upon Scarf's pioneering work. In this section Kuhn's first algorithm is first discussed. Some of the basic ideas and convergence-arguments of this algorithm play a very important role in other fixed point algorithms dicussed later. After Kuhn's algorithm a vector labelling algorithm of Eaves [1971] is treated.

Kuhn's first algorithm on $\mathrm{S}^{\mathrm{n}}$ (see Kuhn [1968]) is an artificial start algorithm in the sense that it needs a layer of artificially labelled points outside $S^{n}$ on which the algorithm starts. Let $U^{n}$ denote aff $S^{n}$. 
DEFINITION 4.3.1. Let $\mathrm{m}^{-1}$ be the grid size of the standard triangulation of $\mathrm{U}^{\mathrm{n}}$. Then

$$
\tilde{S}^{n}=\left\{x \in U^{n} \mid x_{i} \geq 0, i \in I_{n} \text { and } x_{n+1} \geq-m^{-1}\right\}
$$

Moreover,

$$
\tilde{S}_{i}^{n}=\left\{x \in \tilde{s}^{n} \mid x_{i}=0\right\} \quad i=1, \ldots, n
$$

and

$$
\tilde{s}_{n+1}^{n}=\left\{x \in \tilde{s}^{n} \mid x_{n+1}=-m^{-1}\right\}
$$

i.e. $\tilde{s}_{i}^{n}$ is the $i$-th boundary of $\tilde{s}^{n}, i \in I_{n+1}$.

Clearly, $\tilde{s}^{\mathrm{n}}$ is triangulated by the standard triangulation of $\mathrm{u}^{\mathrm{n}}$ restricted to $\tilde{s}^{n}$. Moreover, the grid points of $\tilde{s}^{n}$ outside $s^{n}$ are all points in $\tilde{s}_{n+1}^{n}$. It is easily seen that a grid point $y \in s_{n+1}^{n}$ is a vertex of at least one simplex $\sigma\left(y^{1}, \ldots, y^{n+1}\right)$ such that the facet $\tau\left(y^{1}, \ldots, y^{n}\right)$ is a simplex of the triangulation of $\tilde{s}_{n+1}^{n}$ and $y^{n+1}=y$. The idea of the algorithm is to give the vertices of $\tilde{s}_{n+1}^{n+1}$ an artificial label in such a way that for the a priori chosen grid point $y \in s_{n+1}^{n}$ one of these facets, say $\tau^{0}$, is almost completely labelled in the sense that the vertices of $\tau^{0}$ carry all the labels $1, \ldots, n$. Also the artificial labelling will be such that $\tau^{0}$ is the only almost completely labelled boundary-facet of $\tilde{s}^{n}$. Since $\tau^{0}$ is a boundary-facet, there is only one n-simplex $\sigma^{0}$ containing $\tau^{0}$ as a facet. Note that $\tau^{0}$ is opposite to the vertex $y$ of $\sigma^{0}$. This simplex will be the starting simplex of the algorithm. Now $\ell(y)$ is computed. Since $y \in s_{n+1}^{n}$, $\ell(y) \neq n+1$. Hence $\ell(y)=\ell\left(y^{5}\right)$ for a unique vertex $y^{s}$ of $\tau^{0}$. By replacing $y^{\mathrm{s}}$ we obtain a unique simplex $\sigma^{1}$ adjacent to $\sigma^{0}$ having a new vertex, say $\bar{y}$. If $\ell(\bar{y})=n+1, \sigma^{1}$ is completely labelled. Otherwise we replace the vertex of $\sigma^{1}$ having the same label as $\bar{y}$ to obtain a simplex $\sigma^{2}$ adjlacent to $\sigma^{1}$, etc. So, the algorithm generates a path of adjacent n-simplices $\sigma^{0}, \sigma^{1}, \sigma^{2}, \ldots$ such that two adjacent simplices have a common almost completely labelled facet, until label $\mathrm{n}+1$ is found.

Formally, we choose an arbitrary point $y \in S_{n+1}^{n}$ which must be a grid point of the (standard) triangulation of $s^{n}$. Note that Kuhn chooses $y=\left(n^{-1}, \ldots, n^{-1}, 0\right)^{\top}$ and $m=k n$ for some positive integer $k$. The artificial 
grid points are labelled as follows:

(4.3.1)

$$
\ell(x)=\min \left\{j \mid y_{j}-x_{j}=\min _{k \in I_{n}}\left(y_{k}-x_{k}\right)\right\} \quad x \in \tilde{s}_{n+1}^{n}
$$

Furthermore, a point $\mathrm{x} \in \mathrm{S}^{\mathrm{n}}$ receives a label according to the standard integer labelling rule on $s^{n}$. Obviously, $l(x) \neq i$ if $x \in \tilde{s}_{i}^{n}$ since $\ell(x) \neq n+1$ if $x \in \tilde{s}_{n+1}^{n}$ and $\ell(x) \neq i$ if $x \in s_{i}^{n}$ or $x \in \tilde{s}_{n+1}^{n} n \tilde{s}_{i}^{n}, i=1, \ldots, n$. Observe that $\tilde{s}_{i}^{n}=\left(\tilde{s}_{i}^{n} \cap \tilde{s}_{n+1}^{n}\right) \cup s_{i}^{n}, i=1, \ldots, n$. Let $\tau^{0}\left(y^{1}, \pi\right)$ be the $(n-1)-$ simplex such that

$$
\mathrm{y}^{1}=\mathrm{y}-\mathrm{m}^{-1} \sum_{i=1}^{\mathrm{n}} \mathrm{q}(\mathrm{i}) \quad \text { and } \pi_{i}=i, i=1, \ldots, \mathrm{n}-1
$$

Clearly, the vertices $y^{1}, \ldots, y^{n}$ of $\tau^{0}$ are grid points of $\tilde{s}_{n+1}^{n}$ since $y^{i+1}=y^{i}+m^{-1} q(i), i \in I_{n-1}$, and $y \in s_{n+1}^{n}$. Moreover, $\tau^{0}$ is almost completely labelled, since $l\left(y^{i}\right)=i, i \in I_{n}$. Because of the linear structure of the artificial labelling on $\tilde{S}_{n+1}^{n^{n}}$, it is easily seen that $\tau^{0}$ is the only almost completely labelled simplex in $\tilde{S}_{n+1}^{n}$. Further, since $\ell(x) \neq i$ if $x \in \tilde{S}_{i}^{n}, \tilde{S}_{i}^{n}$ cannot contain an almost completely labelled simplex, $i \in I_{n}$. So, we have the following result.

COROLLARY 4.3.2. The $(n-1)$-simplex $\tau^{0}\left(y^{1}, \pi\right)$ is the only almost completely labelled boundary-facet of $\tilde{\mathrm{s}}^{\mathrm{n}}$.

Next, let $\sigma^{0}$ be the unique n-simplex of the triangulation having $\tau^{0}$ as facet (theorem 2.2.8). This $\sigma^{0}$ can be represented by $\sigma^{0}=\sigma^{0}\left(y^{1}, \pi\right)$ where $y^{1}$ is defined above and $\pi_{i}=i, i \in I_{n}$. Note that the last vertex of $\sigma^{0}$ is equal to $y$, i.e. $y^{n+1}=y$. The steps of the algorithm are now as follows.

STEP 0. Set $\sigma$ equal to $\sigma^{0}\left(y^{1}, \pi\right)$ and $\bar{y}$ equal to $y^{n+1}$.

STEP 1. Calculate $\ell(\bar{y})$. If $\ell(\bar{y})=n+1, \sigma$ is completely labelled and the algorithm terminates. Otherwise, $\ell(\bar{y})=\ell\left(y^{s}\right)$ for exactly one vertex $y^{s} \neq \bar{y}$. The facet $\tau\left(y^{1}, \ldots, y^{s-1}, y^{s+1}, \ldots, y^{n+1}\right)$ is almost completely labelled.

STEP 2. The simplex $\sigma\left(\mathrm{y}^{1}, \pi\right)$ is adapted according to theorem 2.5 .5 by replacing $\mathrm{y}^{\mathrm{s}}$. Return to step 1 with $\overline{\mathrm{y}}$ equal to the new vertex of $\sigma$. 
We now prove that the algorithm terminates within a finite number of steps with a completely labelled simplex. Since $\tau^{0}$ is the only almost completely labelled simplex in the boundary of $\tilde{S}^{\mathrm{n}}$, all steps are feasible, i.e. each simplex generated in step 2 is a simplex of the standard triangulation of $\tilde{S}^{n}$, unless the algorithm returns in $\sigma^{0}$ while the vertex $y^{n+1}=y$ has to be replaced. However, we will prove that cycling cannot occur. Assume the contrary and let $\sigma^{j}=\sigma^{j}, i<j$, be the first simplex in which the algorithm returns. So, the adjacent simplices $\sigma^{j-1}$ and $\sigma^{i}$ have a common almost completely labelled facet. Since $\sigma^{i}$ has two adjacent simplices sharing an almost completely labelled facet with $\sigma^{i}$ if $i \geq 1$ and only one such a simplex if $i=0$ (see again theorem 2.2.8), $\sigma^{j-1}$ must be equal to $\sigma^{i-1}$ or $\sigma^{i+1}$ if $i \geq 1$, and equal to $\sigma^{1}$ if $i=0$. Since $\sigma^{i}$ is the first revisited simplex, this can only occur if $j-1=i+1$ and hence $\sigma^{i}=\sigma^{i+2}$. This gives a contradiction since according to step 1 the algorithm never goes back. Consequently, a unique path of adjacent different $n$-simplices of the standard triangulation of $\tilde{S}^{n}$ is generated. Since the number of $n$-simplices is finite, the algorithm must terminate, which can only occur if a completely labelled simplex is found (Lemke's argument). Moreover this simplex lies in $s^{n}$ since $l(x) \neq n+1$ if $x \in\left(\tilde{s}_{n+1}^{n} \cup s_{n+1}^{n}\right)$, and is therefore a good approximation of a fixed point. Kuhn's second algorithm is a variable dimension algorithm and starts with the single point e(1). This algorithm is a special case of the variable dimension algorithm of Van der Laan and Talman [1979a] to be discussed in section 5. Both algorithms of Kuhn are characterized by a start on the boundary of $\mathrm{s}^{\mathrm{n}}$ and by a fixed grid size throughout the algorithm. As argued in the introduction these features cause inefficiency. Moreover, Kuhn developed his algorithms only for integer labelling. The first fixed point algorithm based on vector labelling is due to Eaves [1971]. Also this method is characterized by a start outside the region of interest. To describe Eaves' algorithm, let $\mathrm{C}$ be a compact, convex n-dimensional subset of $R^{n}$. Suppose we want to compute a fixed point of an u.s.c. mapping $\phi$ from $C$ to the set of nonempty, convex subsets of C. First, the mapping $\phi$ is replaced by the u.s.c. mapping $\bar{\phi}$ from an n-simplex $S$ containing $C$ in its interior to the set of nonempty, convex subsets of $s$, where $\bar{\phi}$ is defined as in the proof of theorem 2.4.3. Remember that $x^{*}$ is a fixed point of $\phi$ if and only if $\mathrm{x}^{*}$ is a fixed point of $\bar{\phi}$. Let $\mathrm{y}$ be an arbitrarily chosen point on the boundary of $S$ and let $G$ be a triangulation of $S$ such that $y$ is in the interior of an $(n-1)$-facet $\tau^{0}$. Note that $\tau^{0}$ is a 
boundary-facet. Furthermore, let the vector d be equal to $\left((y-c)^{\top}, 0\right)^{\top}+e$, where $c$ is the interior point of $C$ defined in the proof of theorem 2.4.3. Each point $\mathrm{x} \in \mathrm{S}$ receives the label $\ell(x)$ according to the standard labelling rule on $R^{n}$, where $f(x)$ is an arbitrarily chosen but fixed element of $\bar{\phi}(x)$. Observe that $\ell(x)=\left((c-x)^{\top}, 0\right)^{\top}+$ e if $x \in S \backslash c$.

DEFINITION 4.3.3. A k-simplex $\sigma\left(\mathrm{y}^{1}, \ldots, \mathrm{y}^{\mathrm{k}+1}\right)$ with $\mathrm{k}=\mathrm{n}-1$ or $\mathrm{n}$ is almost completely labelled (or almost complete) if the system of $n+1$ linear equations

$$
\sum_{i=1}^{k+1} \lambda_{i} l\left(y^{i}\right)+\mu d=e
$$

has a nonnegative solution $\left(\lambda_{1}^{*}, \ldots, \lambda_{k+1}^{*}, \mu^{*}\right)$.

If the system of equations is nondegenerated, i.e. if the matrix

$$
\left[\ell\left(y^{1}\right), \ldots, \ell\left(y^{k+1}\right), d\right]
$$

has full rank, an almost completely labelled n-simplex has just two solutions with exactly one variable equal to zero (see Allgower and Georg [1980]). Clearly, an n-simplex $\sigma$ is completely labelled if $\mu^{*}=0$ for one of these solutions. Since $y$ is in the interior of $\tau^{0}$, the latter simplex is almost completely labelled with a positive solution $\left(\lambda_{1}^{*}, \ldots, \lambda_{n}^{*}, \mu^{*}\right)$. Because of the linear structure of $\ell(y)$ on bd $s$ it is easily seen that $\tau^{0}$ is the only almost completely labelled boundaryfacet. From this simplex on the algorithm generates now a path of adjacent n-simplices such that the common facets are almost completely labelled. As soon as $\mu^{*}$ becomes zero, a completely labelled simplex is found. The steps of the algorithm are as follows, where $\sigma^{0}$ in step 0 is the unique $n$-simplex having $\tau^{0}\left(y^{1}, \ldots, y^{n}\right)$ as facet.

STEP 0. Set $\sigma$ equal to $\sigma^{0}\left(y^{1}, \ldots, y^{n+1}\right)$ and $s$ equal to $n+1$. STEP 1. Calculate $\ell\left(y^{\mathbf{S}}\right)$ and make a standard linear programming pivot step by bringing $\ell\left(y^{s}\right)$ in the system of linear equations

$$
\sum_{\substack{i=1 \\ i \neq s}}^{n+1} \lambda_{i} l\left(y^{i}\right)+\mu d=e,
$$


to obtain a new feasible solution. If $\mu$ becomes zero, $\sigma$ is completely labelled and the algorithm terminates. Otherwise, $\lambda_{t}$ becomes zero for some $t \neq s$, i.e. $\tau\left(y^{1}, \ldots, y^{t-1}, y^{t+1}, \ldots, y^{n+1}\right)$ is almost completely labelled.

STEP 2. Adapt $\sigma$ by replacing $y^{t}$. Return to step 1 with $y^{s}$ equal to the new vertex of $\sigma$.

Assuming nondegeneracy, all replacement and pivot steps are unique and feasible. Hence, the algorithm can not cycle and must therefore terminate within a finite number of iterations with a completely labelled simplex. We remark that the problem of degeneracy can be solved by applying a lexicographic pivot rule (see Eaves [1971], Todd [1976a] and others).

\subsection{MERRILL'S ALGORITHM.}

In 1971 Merrill introduced a vector labelling algorithm on $\mathrm{R}^{\mathrm{n}}$ which can start anywhere. Like Kuhn's first algorithm, it needs a layer of artificially labelled points. More precisely, Merrill's algorithm applied on $\mathrm{s}^{\mathrm{n}}$ is identical to Kuhn's algorithm on $\mathrm{s}^{\mathrm{n}+1}$. Therefore, we first discuss the application of Merrill's algorithm for integer labelling to compute a fixed point of a continuous function on $\mathrm{s}^{\mathrm{n}}$. (see also Kuhn and Mackinnon [1975] and Mackinnon [1975,1976]). For given grid size $\mathrm{m}^{-1}$, take an arbitrarily chosen gridpoint $\mathrm{y}$ of the standard triangulation of $s^{n}$. Note that $y$ corresponds with the point $\bar{y}=\left(y^{\top}, 0\right)^{\top}$ in $s_{n+2}^{n+1}$. Now each grid point $\left(z^{\top}, 0\right)^{\top}$ of the standard triangulation of $s^{n+1}$ receives the label $\ell(z)$ according to the standard integer labelling rule on $s^{\mathrm{n}}$. A grid point $x$ in $s^{n+1} \backslash s_{n+2}^{n+1}$ receives the label $n+2$, i.e.

$$
\ell(x)=n+2 \text { if } \quad x_{n+2}>0
$$

Note that this labelling rule is proper and that the $n+1$ vertices of a completely labelled $(n+1)$-simplex having a label not equal to $n+2$, are all lying in $s_{n+2}^{n+1}$. Hence, the $n$-facet of a completely labelled simplex in $\mathrm{s}^{\mathrm{n}+1}$ whose vertices carry the labels $1, \ldots, \mathrm{n}+1$ corresponds to a

completely labelled $\mathrm{n}$-simplex in $\mathrm{s}^{\mathrm{n}}$. To find a completely labelled simplex in $s^{n+1}$ Merrill's algorithm operates in exactly the same way as Kuhn's first algorithm described in section 3. First an artificial layer 
$\tilde{s}_{n+2}^{n+1}$ is added, whose vertices are labelled according to (4.3.1) with respect to the point $\bar{y}$. Then the algorithm starts with the $(n+1)-$ simplex $\sigma^{0}\left(y^{1}, \pi\right)$ such that $\pi_{i}=i, i=1, \ldots, n+1$, and $y^{n+2}=\bar{y}$. Again the facet $\tau^{0}\left(y^{1}, \ldots, y^{n+1}\right)$ is the unique almost completely labelled boundary-facet of $\tilde{s}^{\mathrm{n}+1}$. From that simplex a path of adjacent $(\mathrm{n}+1)$-simplices is generated such that the common facets are almost completely labelled. As soon as label $n+2$ is found the algorithm terminates. Following the arguments in section 3 it is easily seen that the algorithm converges. Actually, only simplices of the set conv $\left(\tilde{S}_{n+2}^{n+1} \cup S_{n+2}^{n+1}\right)$ are generated and the algorithm terminates if an almost completely labelled n-simplex of $s_{n+2}^{n+1}$ is found. Therefore the set $\left\{x \in \tilde{s}^{n+1} \mid x_{n+2}>0\right\}$ can be deleted. Assume now that we want to compute a fixed point of an u.s.c. mapping from $S^{\mathrm{n}}$ to the set of nonempty, convex subsets of $s^{n}$. Again the set $\tilde{S}^{n+1}$ is triangulated as described above for some grid size $\mathrm{m}^{-1}$, and an arbitrary grid point $\bar{y} \in s_{n+2}^{n+1}$, which corresponds to $y$ (say) in the set $s^{n}$, is chosen. Now each point of $S_{n+2}^{n+1}$ and $\tilde{S}_{n+2}^{n+1}$ receives an $(n+1)$-vector label. A point $\left(x^{\top}, 0\right)^{\top}$ in $s_{n+2}^{n+1}$ receives the label $\ell(x)$ as stated in definition 4.2.3, where $f(x)$ is an a priori chosen element of $\phi(x)$. A point $\left(z^{\top},-m^{-1}\right)^{\top}$ in $\tilde{s}_{n+2}^{n+1}$ receives label $z-y+e$. Clearly, the $n$-simplex $\tau^{0}$ in $\tilde{s}_{n+2}^{n+1}$ is completely labelled, where $\tau^{0}$ is defined as above. Points $\left(z^{\top} ; \delta\right)^{\top}$ in $s^{n+1}$ with $\delta>0$ are not lahelled (since they are not generated by the algorithm). From the simplex $\tau^{0}$ the algorithm generates in the same way as Eaves' first algorithm a path of adjacent simplices in $\operatorname{conv}\left(\tilde{S}_{n+2}^{n+1} \cup s_{n+2}^{n+1}\right)$ with common completely labelled facets, until a completely labelled facet in $\mathrm{s}_{\mathrm{n}+2}^{\mathrm{n}+1}$ is generated. Clearly this facet yields an approximate fixed point in the corresponding $n$-simplex of $\mathrm{s}^{\mathrm{n}}$. Using standard arguments the algorithm terminates within a finite number of pivot and replacement steps with such a facet. It is now easily seen how to apply Merrill's algorithm on $\mathrm{R}^{\mathrm{n}}$ for integer and vector labelling. First $\mathrm{R}^{\mathrm{n}+1}$ is triangulated according to the KQ triangulation with arbitrarily chosen grid size $\delta$. This induces a triangulation of the subset $R^{n} x[0, \delta]$. Let $y$ now be an arbitrary point in $R^{n}$. By a shift of the grid we can always let the point $\bar{y}=\left(y^{\top}, 0\right)^{\top}$ be a point interior in an $n$-simplex $\tau^{0}$ of the set $R^{n} \times\{0\}$. The latter set will be the artificial level for the algorithm. In case of integer labelling a point $\bar{x}=\left(x^{\top}, 0\right)^{\top}$ in $R^{n} x\{0\}$ receives the artificial label $\ell(\bar{x})$ where 


$$
\ell(\bar{x})=\min \left\{j \mid \bar{y}_{j}-\bar{x}_{j}=\max _{k \in I_{n+1}}\left(\bar{y}_{k}-\bar{x}_{k}\right)\right\} .
$$

Further, a point $\bar{z}=\left(z^{\top}, \delta\right)^{\top}$ in $R^{n} \times\{\delta\}$ receives the standard label $\ell(z)$. Clearly, the simplex $\tau^{0}$ is the unique completely labelled simplex in $\mathrm{R}^{\mathrm{n}} \times\{0\}$ in the sense that all vertices of $\tau^{0}$ are differently labelled. From this boundary-facet on, the algorithm generates in the same way as described above a path of adjacent $(n+1)$-simnlices with completely labelled common facets until a facet is found in $R^{n} \times\{\delta\}$ corresponding to a completely labelled simplex in $\mathrm{R}^{\mathrm{n}}$. Since the number of simplices is finite in each compact region of $R^{n} \times[0, \delta]$, either the algorithm terminates within a finite number of steps or an infinite path to infinity is generated. A convergence condition can be found in Todd [1976a, page 917 . This condition is such that every point outside some bounded region has a neighbourhood of radius $\delta$ in which at least one integer label is excluded, which implies that outside that region never completely labelled simplices can be generated.

In case of vector labelling we take the same triangulation and label a point $\bar{x}=\left(x^{\top}, 0\right)^{\top}$ in $R^{n} \times\{0\}$ artificially by $\ell(\bar{x})=\bar{y}-\bar{x}+$ e. Of course, a point $\bar{z}=\left(z^{\top}, \delta\right)^{\top}$ in $R^{n} \times\{\delta\}$ receives the standard label $\ell(z)$. Again $\tau^{\circ}$ will be the only completely labelled $n$-simplex in $R^{n} \times\{0\}$. From this boundary-facet the algorithm generates by pivot and replacement steps as described for Eaves' first algorithm a path of adjacent (n+1)simplices having common completely labelled facets. As soon as a facet in $\mathrm{R}^{\mathrm{n}} \times\{\delta\}$ is generated, the algorithm terminates. A convergence condition (Merrill's condition) is given in the next theorem. The proof of this theorem can be found in Merrill [1971, 1972].

THEOREM 4.4.1. Suppose there exist $w \in R^{n}, \rho>0$ and $\mu>0$ such that whenever $x \notin B(w, \mu), f \in \phi(x)$ and $z \in B(x, \rho)$

$$
(f-x)^{\top}(w-z)>0 .
$$

Then the algorithm terminates if the grid size of the triangulation is small enough.

Note that Merrill's algorithm can be seen to trace zeroes of a piecewise linear approximation to $h$ with respect to the triangulation of $R^{n} \times[0, \delta I$, 
where $h$ is the homotopy function on $R^{n} \times[0, \delta]$ defined by

$$
h(x, t)=t \delta^{-1}(f(x)-x)+(\delta-t) \delta^{-1}(y-x) \quad 0 \leq t \leq \delta, x \in R^{n} .
$$

Observe that when a completely labelled $n$-simplex $\sigma$ on $R^{n} \times\{\delta\}$ is found, the algorithm can be restarted for a smaller $\delta$ and with the point $y=\Sigma_{i=1}^{n+1} \lambda_{i}^{*} y^{i}$, where the $y^{i}$ 's are the (corresponding) vertices of $\sigma$ on $R^{n}$ and the $\lambda_{i}^{*} ' s, i \in I_{n+1}$, are the solution of the linear system of equations with respect to $\sigma$. Of course, the same holds for the algorithm on $S^{n}$ and for integer labelling. In the latter case the barycenter is a good choice to be the next starting point, i.e. take $\lambda_{i}^{*}=(n+1)^{-1}$.

\subsection{VAN DER LAAN AND TALMAN'S BASIC ALGORITHM.}

As argued in Van der Laan and Talman [1979a,1979b] and Van der Laan [1980], Merrill's algorithm has the disadvantage that it operates with artificially labelled points influencing the path of generated simplices, in particular if simplices are generated far away from the starting point. Moreover, Merrill's algorithm generates $\quad(n+1)$-dimensional simplices. So, if a long path is generated, the algorithm needs many iterations. Also, if a path is generated such that all components have to be decreased with the same amount, many steps have to be performed.

Both for integer and vector labelling Van der Laan and Talman proposed an algorithm which starts with a single point, i.e. a zero-dimensional simplex. This point is an arbitrarily chosen grid point of the (standard) triangulation of $S^{n}$ (or $R^{n}$ ). From that point on, a path of adjacent simplices of variable dimension is generated, until a completely labelled n-simplex is found. As soon as a new label is found the dimension of the current simplex is increased by adding a new vertex to it. Sometimes the dimension is decreased by deleting a label and a vertex. This is to guarantee non-cycling.

First we discuss the method for integer labelling on $S^{n}$. Assume $S^{n}$ is triangulated in the standard way with grid size $\mathrm{m}^{-1}$ and let $\mathrm{y}$ be an arbitrary grid point, which will be the starting point. As usual a point $\mathrm{x} \in \mathrm{S}^{\mathrm{n}}$ receives a label according to the standard labelling rule.

DEFINITION 4.5.1. Let $T$ be a proper subset of $I_{n+1}$ and let $|T|=t$. A (t-1)-simplex $\sigma$ is $T$-complete if the $t$ vertices of $\sigma$ carry all the labels 
of the set $\mathrm{T}$.

Note that the starting point $y$ is $\{l(y)\}$-complete. Next, let for $i \in I_{n} q(i)$ be the i-th column of the standard triangulation matrix $\Omega$ of $S^{n}$ be defined as in section 2.6 and let

$$
q(n+1)=-\sum_{i=1}^{n} q(i) .
$$

Furthermore, $\pi(T)=\left(\pi_{1}, \ldots, \pi_{t}\right)$ denotes a permutation of the $t$ elements of $T$. Moreover, $\sigma\left(\mathrm{y}^{1}, \pi(\mathrm{T})\right)$ is the simplex $\sigma\left(\mathrm{y}^{1}, \ldots, \mathrm{y}^{\mathrm{t}+1}\right)$ such that

$$
y^{i+1}=y^{i}+m^{-1} q\left(\pi_{i}\right) \quad i=1, \ldots, t .
$$

Note that $\sigma\left(y^{1}, \pi(T)\right)$ is a $t$-face of the standard triangulation of $s^{n}$ if $y^{1}$ is a grid point and if all vertices lie in $s^{n}$.

Now the algorithm proceeds as follows:

STEP 0. Set $t=0, T=\varnothing, \pi(T)=\varnothing, y^{1}=y, \sigma=\sigma\left(y^{1}, \pi(T)\right), \bar{y}=y^{1}$ and

$$
R_{i}=0, i \in I_{n+1} \text {. }
$$

STEP 1. Calculate $\ell(\bar{y})$. If $\ell(\bar{y}) \notin T$, a new label is found and go to step 3 . Otherwise $\ell(\bar{y})=\ell\left(y^{S}\right)$ for exactly one vertex $y^{\mathbf{S}} \neq \bar{y}$. The facet $\tau\left(\mathrm{y}^{1}, \ldots, \mathrm{y}^{\mathrm{s}-1}, \mathrm{y}^{\mathrm{s}+1}, \ldots, \mathrm{y}^{\mathrm{t}+1}\right)$ is $\mathrm{T}$-complete.

STEP 2. If $s=t+1$ and $R_{\pi_{t}}=0$, go to step 4. Otherwise $\sigma\left(y^{1}, \pi(T)\right)$ and $R$ are adapted according to table 4.5 .1 by replacing the vertex $y^{\mathrm{S}}$. Return to step 1 with $\bar{y}$ equal to the new vertex of $\sigma$.

STEP 3. If $t=n, \sigma$ is completely labelled and the algorithm terminates. Otherwise increase the dimension. Set $T=T \cup\{\ell(\bar{y})\}$, $\pi(T)=(\pi(T), l(\bar{y})), \sigma=\sigma\left(y^{1}, \pi(T)\right)$ and $t=t+1$. Return to step 1 with $\bar{y}=y^{t+1}$.

STEP 4. Decrease the dimension. Set $T=T /\left\{\pi_{t}\right\}, \pi(T)=\left(\pi_{1}, \ldots, \pi_{t-1}\right)$, $\sigma=\sigma\left(y^{1}, \pi(T)\right)$ and $t=t-1$. Return to step 2 with $y^{s}$ being the vertex of $\sigma$ with label equal to the deleted integer. 


\begin{tabular}{l|l|l|l} 
& \multicolumn{1}{|c}{$y^{1}$ becomes } & $\pi(T)$ becomes & $R$ becomes \\
\hline$s=1$ & $y^{1}+m^{-1} q\left(\pi_{1}\right)$ & $\left(\pi_{2}, \ldots, \pi_{t}, \pi_{1}\right)$ & $R+e\left(\pi_{1}\right)$ \\
$2 \leq s \leq t$ & $y$ & $\left(\pi_{1}, \ldots, \pi_{s-2}, \pi_{s}, \pi_{s-1}, \pi_{s+1}, \ldots, \pi_{t}\right)$ & $R$ \\
$s=t+1$ & $Y^{1}-m^{-1} q\left(\pi_{t}\right)$ & $\left(\pi_{t}, \pi_{1}, \ldots, \pi_{t-1}\right)$ & $R-e\left(\pi_{t}\right)$
\end{tabular}

Table 4.5.1. $\mathrm{s}$ is the index of the vertex to be replaced.

Note that a replacement step of table 4.5.1 corresponds to the standard replacement step in $R^{t}$ (theorem 2.5.5). The vector $R$ of nonnegative integers is such that $R_{i}=0, i \notin T$, and

$$
y^{1}=y+m^{-1} \sum_{j \in T} R_{j} q(j)
$$

Observe that the vector $R$ is only used to test in sten 2 whether a label has to be deleted (step 4) or not. As soon as $R_{i}$ threatens to become negative, which can only occur if the last vertex of $\sigma$ must be replaced and $R_{\pi_{t}}=0$, label $i$ is deleted and the dimension is decreased. So, in view of (4.5.1) it is natural to define for $T$ being a proper set of $I_{n+1}$ the region $A(T)$, where

$$
A(T)=\left\{x \in S^{n} \mid x=y+\sum_{j \in T} \lambda_{j} q(j) \text { for nonnegative } \lambda_{j}, j \in T\right\} .
$$

Clearly, the region $A(T)$ is triangulated by the collection of t-simplices $\sigma\left(y^{1}, \pi(T)\right)$ in $S^{n}$ such that $y^{1}$ satisfies (4.5.1) for nonnegative integers $R_{j}, j \in T$. Note that $\operatorname{dim} A(T)$ is indeed equal to $t$. Hence, the algorithm generates a path of adjacent simplices of variable dimension such that two adjacent t-simplices share a T-complete facet, are of the form $\sigma\left(y^{1}, \pi(T)\right)$ and lie in $A(T)$ for some $T \subset I_{n+1}$. If two adjacent simplices have not the same dimension, i.e. if one of them is a facet of the other, then the (say) $T$-complete facet is of the form $\tau\left(\mathrm{Y}^{1}, \pi(\mathrm{T} /\{j\})\right)$ and lies in $\mathrm{A}(\mathrm{T} /\{j\})$ for some $j \in T$, whereas the simplex is of the form $\sigma\left(y^{1}, \pi(T)\right)$ and lies in $A(T)$. Note that in the latter case $R_{j}=0$ and that $y^{t+1}=y^{t}+m^{-1} q(j)$ is the vertex of $\sigma$ opposite to the facet $\tau$. Roughly speaking, $A^{\prime}(T)$ is the region of $S^{n}$ which lies between the starting point $y$ and the boundaries $S_{i}^{n}$ where label $i$ does not occur, $i \in T$. So, if a new label $j \notin T$ (say) is found, a search is made in $A(T \cup\{j\})$ in the direction of the $j$-th boundary and still close to the other boundaries. In this area of $s^{n}$ 
one may expect to find labels not in the set $T$. By this property it can easily be seen that simplices outside $s^{n}$ cannot be generated. If, however, the generated path of simplices moves away from some boundary $S_{i}^{n} i \in T$, then label $i$ is dejeted as soon as a $(t-1)$-simplex in $A(T \backslash\{i\})$ is generated. Note that this simnlex is T-complete. By replacing the vertex of $\tau$ with label $i$, the algorithm continues in $A(T \backslash\{i\})$, etc. By the proper labelling rule it is easy to see that all other steps are also feasible. Moreover, all steps are unique (see van der Laan and Talman [1979a]) sothat cycling cannot occur. Since the number of simplices in $s^{n}$ is finite, the algorithm must terminate within a finite number of iterations with a completely labelled n-simplex yielding an approximate fixed point. of course, the algorithm can now be restarted with a larger $\mathrm{m}$ and close to the approximation to obtain a better accuracy.

To compute a fixed point of a mapping $\phi$ from $s^{n}$ into itself, each point $x$ is labelled according to the standard labelling rule whereas $S^{n}$ is again triangulated in the standard way for some $\mathrm{m}$.

DEFINITION 4.5.2. Let $T$ be a proper subset of $I_{n+1}$ with $|T|=t$. A $(t-1)-$ simplex $\sigma\left(y^{1}, \ldots, v^{t}\right)$ is called $\mathrm{m}$-complete if the system of $\mathrm{n}+1$ linear equations

(4.5.2) $\sum_{i=1}^{t} \lambda_{i} l\left(y^{i}\right)+\sum_{h \notin T} \mu_{h} e(h)=e$

has a nonnegative solution $\left(\lambda^{*}, \mu^{*}\right)=\left(\lambda_{1}^{*}, \ldots, \lambda_{t^{\prime}}^{*} \mu_{j^{\prime}}^{*} j \notin T\right)$.

Starting with $T=\varnothing$ and with the system $\Sigma_{h=1}^{n+1} \mu_{h} e(h)=e$, the algorithm generates from an arbitrarily chosen grid point $y$ by pivot and replacement steps a path of adjacent simplices of variable dimension such that two adjacent $t$-simplices whose common facet is T-complete are simplices of the form $\sigma\left(y^{1}, \pi(T)\right)$ and are lying in $A(T)$. As soon as by a pivot step a unit vector $e(j), j \notin T$, (say) is eliminated (i.e. when $\mu_{j}$ becomes zero), the dimension of the current simplex is increased and a search is made with $(t+1)$-simplices in $A(T \cup\{j\})$ having (TU\{j\})-complete common facets. If, however, the dimension has to be decreased since for some $i \in T$ the region $A(T /\{i\})$ is met, the unit vector $e(i)$ is reintroduced in the system, etc. Thus the algorithm proceeds as follows. 
STEP 0. Set $t=0, T=\varnothing, \pi(T)=\varnothing, y^{1}=y, \sigma=\sigma\left(y^{1}, \pi(T)\right), s=1$ and $R_{i}=0, i \in I_{n+1}$.

STEP 1. Calculate $\ell\left(y^{S}\right)$. Perform a pivot step by bringing $\ell\left(y^{S}\right)$ in the system

$$
\sum_{\substack{i=1 \\ i \neq s}}^{t+1} \lambda_{i} l\left(y^{i}\right)+\sum_{h \notin T} \mu_{h} e(h)=e .
$$

If $\mu_{h}=0$ for all $h$, a completely labelled simplex is found and the algorithm terminates. Otherwise, either $e(j)$ for some $j \notin T$ is eliminated and go to step 3 , or $\ell\left(y^{k}\right)$ is eliminated for exactly one vertex $y^{k} \neq y^{s}$. The facet $\tau\left(y^{1}, \ldots, y^{k-1}, y^{k+1}, \ldots, y^{t+1}\right)$ is T-complete.

STEP 2. If $k=t+1$ and $R_{\pi_{t}}=0$, go to step 4. Otherwise $\sigma\left(y^{1}, \pi(T)\right)$ and $R$ are adapted according to table 4.5 .1 by replacing $\mathrm{y}^{\mathrm{k}}$. Return to step 1 with $s$ equal to the index of the new vertex of $\sigma$.

STEP 3. Increase the dimension. Set $T=T \cup\{j\}, \pi(T)=(\pi(T), j)$, $\sigma=\sigma\left(y^{1}, \pi(T)\right)$ and $t=t+1$. Return to step 1 with $s=t+1$.

STEP 4. Decrease the dimension. Set $T=T \backslash\left\{\pi_{t}\right\}, \pi(T)=\left(\pi_{1}, \ldots, \pi_{t-1}\right)$, $\sigma=\sigma\left(\mathrm{y}^{1}, \pi(\mathrm{T})\right), t=t-1$ and perform a pivot step by bringing $e(r)$ in the system of linear equations ( $r$ is the deleted index)

$$
\sum_{i=1}^{t+1} \lambda_{i} l\left(y^{i}\right)+\sum_{\substack{h \notin T \\ h \neq x}} \mu_{h} e(h)=e .
$$

If for some $j \notin T e(j)$ is eliminated, go to step 3. Otherwise return to step 2 with $k$ equal to the index of the vertex whose label $\ell\left(y^{k}\right)$ is eliminated.

Assuming nondegeneracy, the algorithm terminates within a finite number of steps if all steps are feasible. In Van der Laan[1980] it is shown that both the replacement, pivot and extension steps are feasible. These properties will also be shown in a more general framework in chapter 6 . Thus, a completely labelled simplex will be found by the above algorithm. Clearly, the algorithm can be applied by using other triangulation matrices than Q. In chapter 5 we return to this matter. The algorithm on $S^{\mathrm{n}}$ is illustrated for integer labelling in figure 4.5 .1 for $\mathrm{n}=2$, $\mathrm{m}=9$ and $\mathrm{y}=(3,5,1) / 9$. 


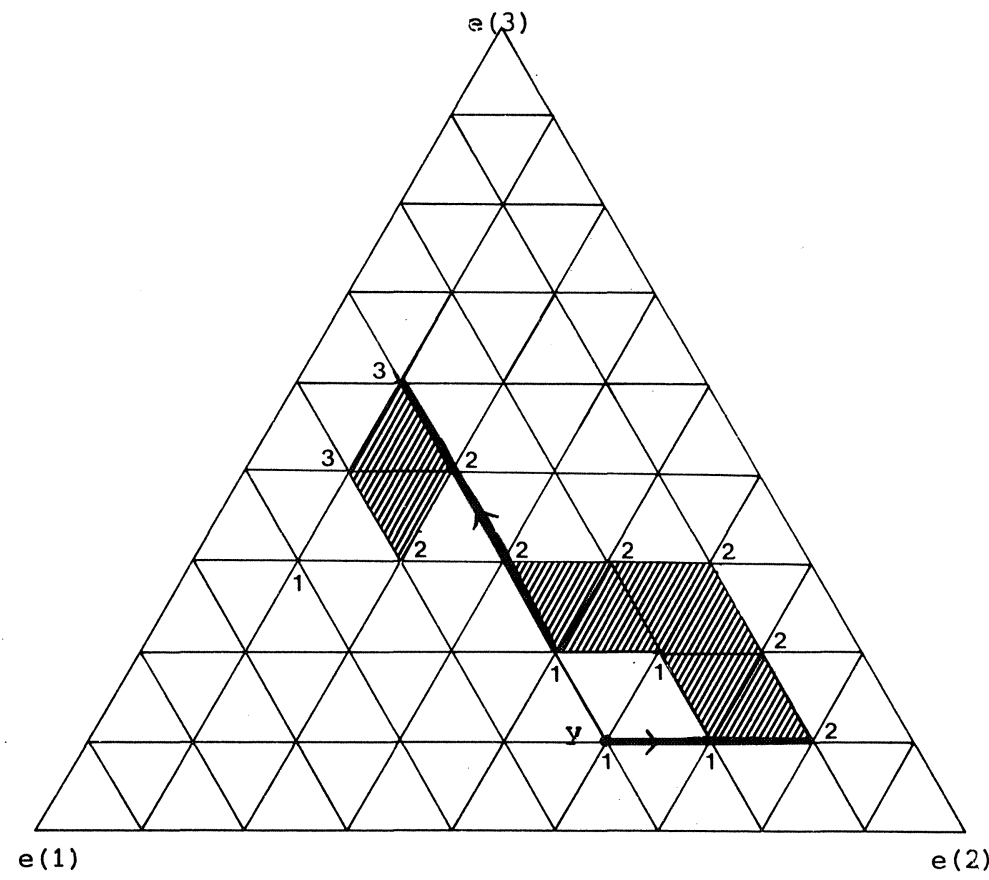

Figure 4.5.1. Van der Laan and Talman's basic algorithm on $S^{\mathrm{n}}$.

The application of Van der Laan and Talman's basic algorithm on $R^{n}$ is straightforward and will not be discussed here (see Van der Laan and Talman [1979b] and Van der Laan [1980]). In chapter 7 a class of variable dimension algorithms on $\mathrm{R}^{\mathrm{n}}$ is presented. It will appear that the basic algorithm is one of the two extreme cases of this class. Also (new) convergence results are given in chapter 7 .

In 1972 Eaves and Eaves and Saigal introduced a homotopy algorithm to compute a Kakutani-fixed point on $S^{n}$ and $R^{n}$ respectively. In their method they use a triangulation of $S^{n} \times[1, \infty)$ (and $R^{n} \times[0, \infty)$ ) with continuous refinement of the grid size with a factor of incrementation of (at most) two. In this kind of algorithms the mapping $\phi$ is deformed from a linear function $\phi^{\circ}$ on the first level to a pjecewise linear approximation $\phi^{\mathrm{k}}$ to $\phi$ with respect to the triangulation of $S^{n}\left(P^{n}\right)$ on level $k$. On level $k$ the grid size is (at most) two times smaller than on level $k-1, k=2,3, \ldots$. For $\mathrm{k}$ goes to infinity fixed points of $\phi^{\mathrm{k}}$ converge to fixed points of $\phi$. The triangulation of the set $s^{n} \times[1, \infty)\left(R^{n} \times[0, \infty)\right)$ is such that it is built up from triangulations between two levels so that there are only grid points on the levels. The grid points on the first level are 
artificially labelled as in Merrill's algorithm, whereas grid points on the other levels are labelled in the standard way. Starting now with the unique completely labelled simplex on the first level, the algorithm generates by pivot and replacement steps a path of adjacent $(n+1)-$ simplices of the triangulation such that two adjacent simplices have common completely labelled $n$-facets. Since on $S^{n}$ the labelling is proper it can easily be seen that simplices outside $S^{n} \times[1, \infty)$ cannot be generated. Moreover, since all steps are unique, cycling cannot occur. Therefore the algorithm finds within a finite number of iterations a completely labelled $n$-simplex on each level $k, k=1,2, \ldots$ If the homotopy-algorithm is apnlied on $\mathrm{n}^{\mathrm{n}} \times[0, \infty)$, either we have the above situation or a path going to infinity is generated. Convergence conditions can be found in Eaves and Saigal [1972]. However, these methods have the disadvantage that the factor of incrementation is at most two whereas a restart algorithm can take any factor. Van der Laan and Talman [1980b], Van der Heyden [1979] and Shamir [1979] independently introduced a homotopy algorithm such that the factor of incrementation can be of any size (see also Van der Laan [1980]). The basic idea for triangulating $S^{n} \times[1, \infty)$ or $R^{n} \times[0, \infty)$. with a general factor was obtained from an interpretation of Van der Laan and Talman's basic algorithm with an artificial level of $\mathrm{n}+1$ points. This interpretation was independently found by Van der Laan and Talman [1979a], Todd [1978b] and Bárány [private communication] (see also chapter 8 ). 
THE EFFICIENCY OF TRIANGULATIONS

\subsection{INTRODUCTION}

Computational experience with several fixed point algorithms has shown that the efficiency of an algorithm is very sensitive to the underlying triangulation (see e.g. Saigal [1977a] and Todd [1978a]). In this chapter several measures for the efficiency of a triangulation are discussed and compared.

In section 2 an intuitively attractive class of so called "fixed point triangulations" of $\mathrm{R}^{\mathrm{n}}$ is introduced. Section 3 discusses two crude measures for triangulations of the unit cube. In section 4 the average directional density of a triangulation is treated. This measure was introduced by Todd to compare triangulations of $\mathrm{R}^{\mathrm{n}}$. The SV-measure developed by Van der Laan and Talman [1980a] is presented in section 5. This measure is based on the ratio of the sum of the squared lengths of the one-faces and the volume of the simplices of the triangulation. The measure is closely related to the DV-measure independently found by Todd. For both measures the (same) optimal triangulation within the class of fixed point triangulations of $\mathrm{R}^{\mathrm{n}}$ will be calculated. Also some of the properties of the optimal triangulation are discussed in section 5. It will appear that this triangulation is a generalization of the equilateral triangulation of $\mathrm{R}^{2}$. In section 6 the average directional density and the SV-measure are calculated for several interesting triangulations. Finally, section 7 presents a new triangulation of the affine hull of $s^{n}$ having the same nice properties as the optimal triangulation of $R^{n}$. In the last two sections also some computational experience is given.

This chapter is based on the work of van der Laan and Talman [1980a]. 
5.2. FIXED POINT TRIANGULATIONS OF $\mathrm{R}^{\mathrm{n}}$.

If a triangulation induces many shapes of long and skinny simplices, a fixed point algorithm suffers from inefficiency (on the average) since the accuracy of an approximation depends on the distance between the vertices of the triangulation while the number of iterations to find completely labelled simplices depends on the number of simplices per unit cube. Therefore it is in the first place worthwile to consider regular, congruent triangulations.

DEFINITION 5.2.1. A triangulation is congruent if all its simplices are congruent to each other.

Observe that the $\mathrm{K}$ triangulation defined in section 2.5 is congruent but that the $\mathrm{H}$ triangulation is not $(\mathrm{n} \geq 3)$. Since for a congruent triangulation it is in general sufficient to derive properties of the simplices for only a single simplex, we make often use of a special simplex having the simplest representation. This simplex makes however only sense if the triangulation is regular, i.e. if the triangulation is of the form AK where $A$ is a nonsingular nxn matrix.

DEFINITION 5.2.2. For a congruent $A K$ triangulation of $R^{n}$ the standard simplex, to be denoted by $s_{n}$ or $s\left(y^{1}, \ldots, y^{n+1}\right)$, is the $n-\operatorname{simplex} \sigma\left(y^{1}, \pi\right)$ such that $y^{1}$ is the zero-vector and $\pi_{i}=i, i=1, \ldots, n$.

Note that the $i^{\text {th }}$ vertex of $s\left(y^{1}, \ldots, y^{n+1}\right)$ is equal to $y^{i}=\Sigma_{j=1}^{i-1} a(j)$, $i=2, \ldots, n+1$.

Although each simplex of a congruent regular triangulation has the same properties, not each such a triangulation is suitable for use in a fixed point algorithm. In view of Merrill's condition, we clearly have that if a point has label $i$ there is an indication that the $i$-th component must be strictly increased whereas the other components must be decreased or remain the same, $i \in I_{n}$. Intuitively, these amounts must be the same for all $i$. So, we can represent the $i-t h$ column of a suitable triangulation matrix by the n-vector

$$
\bar{a}(i)=(\beta, \ldots, \beta, \alpha, \beta, \ldots, \beta)^{\top}
$$


for some positive $\alpha$ and nonpositive $\beta$, where $\alpha$ is on the $i$-th place, $i \epsilon I_{n}$. So, let $\bar{A}$ be the matrix with $i-t h$ column equal to $\bar{a}(i)$. Then we have the following property which follows immediately from the structure of $\bar{A}$.

LEMMA 5.2.3. For an nxn matrix $\overline{\mathrm{A}}$ with $\alpha>0$ and $\beta \leq 0$ holds that the $\overline{\mathrm{A} K}$ triangulation is congruent.

Next consider the vector $y^{n+1}-y^{1}$ of an arbitrary simplex $\sigma\left(y^{1}, \ldots, y^{n+1}\right)$ of an $\overline{A K}$ triangulation. All components of this vector are equal to $\alpha+(n-1) \beta$ since $y^{n+1}-y^{1}=\sum_{i=1}^{n} a(i)$. But, in case $\alpha+(n-1) \beta>0$, the vector $y^{1}-y^{n+1}=-\{\alpha+(n-1) \beta\}$ e can be used to represent the direction to make a search if label $\mathrm{n}+1$ is found since decreasing all components with the same amount corresponds to a search for other labels if a point is found carrying label $\mathrm{n}+1$ (see Van der Laan and Talman [1980a] and Van der Laan [1980]). Therefore we now have a class of triangulations which seems to be suitable for use in a fixed point algorithm.

DEFINITION 5.2.4. The class $A$ of $(\alpha, \beta)$-triangulations is the subclass of $\bar{A} K$ triangulations such that

$$
\beta \leq 0 \text { and } \alpha+(n-1) \beta>0 \text {. }
$$

Note that if $\beta=0$, we obtain the $\mathrm{K}$ triangulation with grid size $\alpha$. If $\beta<0$, the mesh of the $(\alpha, \beta)$-triangulation is $|\beta|$ times the mesh of the $(\alpha /|\beta|),-1)$-triangulation. So, to compute the mesh of an arbitrary $(\alpha, \beta)$-triangulation, we can restrict ourselves to the class of $(\alpha,-1)-$ triangulations. An element of this class will be denoted by $\alpha$-triangulation. Note that $\alpha>\mathrm{n}-1$.

To compute the mesh of an $\alpha$-triangulation, let the number $b$ be equal to

$$
b=\left\lfloor(\alpha+1)^{2} /(4+4 \alpha-2 n)\right\rfloor
$$

where $\lfloor x\rfloor$ denotes the largest integer smaller than or equal to $x$. Since $\alpha>n-1, b$ is positive. 
THEOREM 5.2.5. The mesh of an $\alpha$-triangulation, to be denoted by mesh $A(\alpha)$, is

$$
\operatorname{mesh} A(\alpha)=\left[k\{\alpha-(k-1)\}^{2}+(n-k) k^{2}\right]^{\frac{1}{2}}
$$

where

$$
k= \begin{cases}n & \text { if } b \geq n \\ b & \text { if } b \leq n-1 \text { and }|| y^{b+1}\|\geq\| y^{b+2} \| \\ b+1 & \text { if } b \leq n-1 \text { and }|| y^{b+1}||<\left\|y^{b+2}\right\|\end{cases}
$$

where $y^{j}$ is the $j-t h$ vertex of the standard simplex $s\left(y^{1}, \ldots, y^{n+1}\right)$ of the $\alpha$-triangulation.

PROOF. Since an $\alpha$-triangulation is congruent, we have that

$$
\operatorname{mesh} A(\alpha)=\operatorname{diam} s\left(y^{1}, \ldots, y^{n+1}\right)
$$

Moreover, because of the special structure of the triangulation matrix it is easily seen that

$$
\operatorname{diam} s\left(y^{1}, \ldots, y^{n+1}\right)=\max _{i \in I_{n}}|| y^{i+1}||
$$

Clearly, $y_{j}^{i+1}=\alpha-(i-1)$ for $j=1, \ldots, i$, and $y_{j}^{i+1}=-i$ for $j=i+1, \ldots, n$. Therefore, for $i=1, \ldots, n$

$$
\left\|y^{i+1}\right\|^{2}=i\{\alpha-(i-1)\}^{2}+(n-i) i^{2}=(\alpha+1)^{2} i-(2 \alpha+2-n) i^{2}
$$

The theorem now follows immediately by determining the index $\mathrm{k}$ for which ||$y^{k+1}||$ is maximal. Remember that $y^{1}$ is the zero-vector.

Note that when $\alpha+\infty$, the mesh of the $\left(1,-\alpha^{-1}\right)-t$ triangulation converges to mesh $\sqrt{n}$ of the $\mathrm{K}$ triangulation.

Finally, we give some properties of the triangulation matrix of an $(\alpha, \beta)$-triangulation. These properties will be used in the next sections. 
LEMMA 5.2.6. Let $A(\alpha, \beta)$ be the triangulation matrix of the $(\alpha, \beta)$-triangulation and let $B=\left(b_{i j}\right)$ be the inverse of $A(\alpha, \beta)$. Then

$$
\operatorname{det} A(\alpha, \beta)=\{\alpha+(n-1) \beta\} \quad(\alpha-\beta)^{n-1}
$$

and

$$
\begin{array}{ll}
b_{i i}=\{\alpha+(n-2) \beta\} /[(\alpha-\beta)\{\alpha+(n-1) \beta\}] & i=1, \ldots, n \\
b_{i j}=-\beta /[(\alpha-\beta)\{\alpha+(n-1) \beta\}] & j \neq i .
\end{array}
$$

Before we introduce a measure to determine the optimal triangulation within the class of $\alpha$-triangulations we discuss some crude measures earlier studied in the literature.

\subsection{CRUDE MEASURES.}

The first measure for the efficiency of a triangulation was to count the number of simplices in which the unit cube is triangulated given that the mesh of the triangulation is equal to $\sqrt{\mathrm{n}}$. However, both the $\mathrm{K}-, \mathrm{H}-$ and $\mathrm{J}$ triangulations subdivide the unit cube in $\mathrm{n}$ ! simplices, i.e. this measure does not distinguish between these triangulations. Saigal [1977a] experienced that in particular for large $\mathrm{n}$ the $\mathrm{K}$ triangulation performs much better than the $\mathrm{H}$ triangulation in the sense that the number of iterations for the $\mathrm{K}$ triangulation is much lower than for the $\mathrm{H}$ triangulation if the same algorithm is applied and the same grid size is used. This fact is not unexpected since the $\mathrm{H}$ triangulation gives many shapes of simplices whereas the $\mathrm{K}$ triangulation is congruent and is an element of the class A. Therefore, the above measure is too rough and must be rejected. Before introducing a more sophisticated measure we mention the interesting problem of finding the triangulation of the unit cube into the fewest number of simplices. This is still an open problem. However for $n=3,4$ and 5, Mara [1972] discovered triangulations of the unit cube producing 5,16 and 68 simplices respectively. 
A second measure was given by Saigal, Solow and Wolsey [1975]. Also this measure can only be used to compare triangulations of the unit cube. While the number of simplices of the unit cube is an "n-dimensional" measure, the measure of Saigal et al. is an "1-dimensional" one predicting better the number of iterations of an algorithm. Roughly speaking, the measure estimates the effort (number of iterations) to proceed through the unit cube. To discuss this measure, let $\sigma_{1}, \sigma_{2}, \ldots, \sigma_{k}$ be a sequence of distinct n-simplices such that $\sigma_{i}$ and $\sigma_{i+1}$ are adjacent for $i=1, \ldots, k-1$. Let $\tau_{0}$ be a facet of $\sigma_{1}$ such that $\tau_{0} \neq \sigma_{1} \cap \sigma_{2}$ and let $\tau_{k}$ be a facet of $\sigma_{k}$ such that $\tau_{\mathrm{k}} \neq \sigma_{\mathrm{k}-1} \cap \sigma_{\mathrm{k}}$. Then we call $\sigma_{1}, \ldots, \sigma_{\mathrm{k}}$ a path from $\tau_{0}$ to $\tau_{\mathrm{k}}$ having length $k$. In general let $p(\tau, \bar{\tau})$ be the minimal path-length between two facets $\tau$ and $\bar{\tau}$. For a triangulation $G$ of the unit cube, saigal et al. [1975] defined the following so-called diameter measure, to be denoted by dia $G$.

DEFINTION 5.3.1. The diameter of a triangulation $G$ of the unit cube $C^{\prime}$ is the maximum of the minimal path-length between any pair of boundaryfacets of $G$, i.e.

$$
\operatorname{dia} G=\max _{\tau, \bar{\tau} \in \mathrm{bd} C^{\prime}} \mathrm{p}(\tau, \bar{\tau}) .
$$

Saigal $[1977 a]$ stated that dia $H \geq O\left(n^{3}\right)$ and dia $K=\frac{1}{2} n(n-1)+1$. His compuational experiences with Merrill's algorithm confirmed the theoretical superiority of the $\mathrm{K}$ triangulation over the $\mathrm{H}$ triangulation. As argued by Todd [1976b] the diameter of a triangulation (of the unit cube) is a "worst best case" measure. Moreover, the measure can be only applied to triangulations of the unit cube. Therefore, a more sophisticated measure, called the average directional density of a triangulation, was proposed by Todd [1976a, 1976b and 1978a].

\subsection{AVERAGE DIRECTIONAL DENSITY.}

Before defining the average directional density (a.d.d.) of a triangulation $G$ of $R^{n}$, to be denoted by $N(G)$, we formulate the directional density of $G$ for a given direction d. Roughly said, the directional density of a triangulation is the number of simplices met 
per unit length by a straight line into the direction d. An important fact is that the directional density is not restricted to a triangulation of the unit cube. Observe that the value of the directional density corresponding to the worst direction is again a "worst best case" measure. By averaging the directional density over all directions we obtain the a.d.d..

Formally, let $G$ be a triangulation of $R^{n}$. Then, for $x, d \in R^{n}$ and $t \geq 0$, define the ratio $N(G, x, d, t)$ as the number of simplices of $G$ intersecting the line segment $[x, x+t d]$ divided by the number $t$. Now, denote by $N(G, d, t)$ the limit as $r \rightarrow \infty$ of the average of $N(G, x, d, t)$ for $x$ uniformly distributed in $\left\{y \in R^{n}|||y| \mid \leq r\right\}$, provided this limit exists. Finally, the directional density with direction $d$, to be denoted by $N(G, d)$, is the limit of $N(G, d, t)$ for $t \rightarrow \infty$, provided it exists. Observe that by averaging $x$ lying in a large ball we eliminate the effects of the starting point and that by letting $t \rightarrow \infty$ any effects of the ending point are eliminated. Todd [1976b] proved the following theorem.

THEOREM 5.4.1. For the $\mathrm{K}$-and J triangulations (with grid size 1) holds that all limits exist. Moreover,

$$
N(K, a)=\sum_{j=1}^{n}\left|a_{j}\right|+\sum_{j=1}^{n} \sum_{i=1}^{j-1}\left|a_{i}-a_{j}\right|
$$

and

$$
N(J, d)=\sum_{j=1}^{n}\left|a_{j}\right|+\sum_{j=1}^{n} \sum_{i=1}^{j-1} \frac{1}{2}\left\{\left|d_{i}+d_{j}\right|+\left|a_{i}-d_{j}\right|\right\} .
$$

It can easily be seen that $N(A G, d)=N\left(G, A^{-1} d\right)$ for every non-singular matrix A. Hence, the directional density of an AK- or AJ triangulation can be obtained directly from this theorem.

DEFINITION 5.4.2. The average directional density $N(G)$ of a triangulation $G$ of $R^{n}$ is the average of $N(G, d)$ for $d$ uniformly distributed on the unit ball $\left\{y \in R^{n}|||y| \mid=1\right\}$. 
THEOREM 5.4.3. For both the $\mathrm{K}-$ and $\mathrm{J}$ triangulation holds that

$$
N(K)=N(J)=\left\{n+n(n-1 \gamma \sqrt{2}\}_{g_{n}}\right.
$$

where $g_{n}=2 \Gamma\left(\frac{1}{2} n\right) /\left\{(n-1) \sqrt{\pi} \Gamma\left(\frac{1}{2}(n-1)\right)\right\}$.

Moreover, let $A$ be a nonsingular $n \times n$ matrix and let $b(j)$ be the $j-$ th row of the inverse $B$ of $A$. Then,

$$
N(A K)=\left\{\sum_{j=1}^{n}|| b(j)||+\sum_{j=1}^{n} \sum_{i=1}^{j-1}|| b(i)-b(j) \|\right\} g_{n}
$$

and

$$
N(A J)=\left\{\sum_{j=1}^{n}\|b(j)\|+\sum_{j=1}^{n} \sum_{i=1}^{j-1}\right.
$$

The proof of this theorem can be found in Todd [1976b, 1978a].

Remember that the $\mathrm{H}$ triangulation is identical to the $\widetilde{Q K}$ triangulation as stated in section 2.5 .

COROLLARY 5.4 .4

$$
N(H)=\left\{\sum_{j=1}^{n}(n-j+1) \sqrt{j}\right\} g_{n} .
$$

Using lemma 5.2.6 we can also easily calculate the a.d.d. of an $(\alpha, \beta)$-triangulation, to be denoted by $N(\alpha, \beta)$.

C.PROLLARY 5.4 .5 .

$$
\begin{aligned}
N(\alpha, \beta)= & g_{n}\left[n\left\{\alpha^{2}+2(n-2) \alpha \beta+\left(n^{3}-3 n+3\right) \beta^{2}\right\}^{\frac{1}{2}}+\right. \\
& n(n-1)\{\alpha+(n-1) \beta\} / \sqrt{2}] /[(\alpha-\beta)\{\alpha+(n-1) \beta\}] .
\end{aligned}
$$

Clearly, given the mesh, a triangulation having a low a.d.d. seems to be suitable for use in a fixed point algorithm. For a given AK (or AJ) triangulation it is not hard to calculate $\mathrm{N}(\mathrm{AK})$ (or $\mathrm{N}(\mathrm{AJ})$ )) However, to find a triangulation which minimizes the a.d.d. seems to be a 
difficult problem since the a.d.d. depends on the mesh, i.e. to compare different triangulations wehave to normalize them such that they have the same mesh. But the mesh of an arbitrary triangulation is hard to handle, also if we restrict ourselves to AK triangulations. Thus, an optimal triangulation according to the a.d.d. measure is not known, even not in the subclass of $(\alpha, \beta)$-triangulations. However, Todd [1978a] gave a lower bound for the a.d.d. of an AK triangulation.

THEOREM 5.4.6. For any nonsingular matrix A

$$
\mathrm{N}(\mathrm{AK})>\left(4 \mathrm{n}^{2} / 25 \sqrt{5}\right) \mathrm{g}_{\mathrm{n}} \text { if mesh } \mathrm{AK}=\sqrt{\mathrm{n}} \text {. }
$$

This result was improved by Van der Laan and Talman [1978a] for the class A of $(\alpha, \beta)$-triangulations.

THEOREM 5.4.7. For any $(\alpha, \beta)$-triangulation having mesh equal to $\sqrt{\mathrm{n}}$ holds

$$
\mathrm{N}(\alpha, \beta)>\left\{\frac{1}{2}(\mathrm{n}-1)+(\mathrm{n}-1)^{2} / \sqrt{8}\right\} \mathrm{g}_{\mathrm{n}}
$$

$\underline{\text { PROOF. Let }} \mathrm{y}^{i}$ be the vertices of the standard simplex $s_{n}$ of an $(\alpha, \beta)-$ triangulation having mesh equal to $\sqrt{n}$. We first show that $\alpha \leq 2$. Assume to the contrary that $\alpha>2$. Distinguish now between the cases $0 \geq \beta>-(n-1)^{-1}$ and $-(n-1)^{-1} \geq \beta>-\alpha(n-1)^{-1}$. Note that we have $-\alpha(n-1)^{-1}<\beta$ since $\alpha+(n-1) \beta>0$. If $0 \geq \beta>-(n-1)^{-1}$, then $\alpha+(n-1) \beta>1$ which implies that

$$
\operatorname{mesh} A K=\operatorname{diam} s_{n} \geq\left\|y^{n+1}\right\|=\{\alpha+(n-1) \beta\} \sqrt{n}>\sqrt{n}
$$

contradicting the fact that mesh $A K=\sqrt{n}$. In the other case there exists an integer $k, 1 \leq k \leq n-1$, such that $k \beta \leq-1$ and $(k-1) \beta>-1$. Hence, $\alpha+(k-1) \beta>1$ and $-k \beta \geq 1$ so that

$$
\text { mesh } A K=\operatorname{diam} s_{n} \geq|| y^{k+1} \|=\left[k\{\alpha+(k-1) \beta\}^{2}+(n-k) k^{2} \beta^{2}\right]^{\frac{1}{2}}>\sqrt{n}
$$

again contradicting the fact that mesh $A K=\sqrt{n}$. Hence $\alpha \leq 2$. From lemma 5.2.5 we have for the $i-t h$ row $b(i)$ of $A^{-1}, i \in I_{n^{\prime}}$ that, 
68

$$
\begin{aligned}
\|b(i)\|^{2} & =\left[\{\alpha+(n-2) \beta\}^{2}+(n-1) \beta^{2}\right] /\left[(\alpha-\beta)^{2}\{\alpha+(n-1) \beta\}^{2}\right] \\
& =(\alpha-\beta)^{-2}\left[1+\beta\{\beta-\alpha-(\alpha+(n-1) \beta)\} /\{\alpha+(n-1) \beta\}^{2}\right] \\
& \geq(\alpha-\beta)^{-2}
\end{aligned}
$$

since $\beta \leq 0, \alpha>0,0<\alpha+(n-1) \beta \leq 1$.

Therefore, ||$b(i)|| \geq(\alpha-\beta)^{-1}$ for all $i$. Clearly, ||$b(i)-b(j)||=(\alpha-\beta)^{-1} \sqrt{2}$ for all $i \neq j$. So, from theorem 5.4.3

$$
N(\alpha, \beta) \geq g_{n}\left(n+\left(\frac{n}{2}\right) \sqrt{2}\right) /(\alpha-\beta) .
$$

Finally, $\alpha+(n-1) \beta>0$ and $0<\alpha \leq 2$ imply

$$
\alpha-\beta<n \alpha /(n-1) \leq 2 n /(n-1) .
$$

Hence,

$$
\mathrm{N}(\alpha, \beta)>\left\{\frac{1}{2}(\mathrm{n}-1)+(\mathrm{n}-1)^{2} / \sqrt{8}\right\} \mathrm{g}_{\mathrm{n}^{\prime}}
$$

which proves the theorem.

COROLLARY 5.4.8. Within the class $A$ the lower bound of the a.d.d. converges to $g_{n} n^{2} / \sqrt{8}$ as $n \rightarrow \infty$.

It is still an open problem whether theorem 6.4.8 holds for all (regular) triangulations. Since an $(\alpha, \beta)$-triangulation yields congruent simplices, one would expect that an optimal triangulation is an $(\alpha, \beta)$-triangulation. In the next section we present a measure for which it is indeed possible to calculate the optimal triangulation within the class A. Moreover, we can prove that for $\mathrm{n} \rightarrow \infty$ the a.d.d. of this triangulation converges to the lower bound of corollary 5.4.8. 
5.5. MEASURES BASED ON MINIMIZING THE NUMBER OF GRID POINTS.

In this section a measure is presented which is insensitive for the mesh of a triangulation. Within the class $A$ of fixed point triangulations it will be rather easy to calculate the optimal triangulation. However, before defining this measure, we make some introductory remarks. Intuitively it is obvious that the most efficient fixed point triangulation of $\mathrm{R}^{2}$ is the equilateral $\alpha$-triangulation, i.e. with $\alpha=2+\sqrt{3}$. Note that the 3 one-faces of any simplex of this triangulation have the same length. Unfortunately, for $n \geq 3$ there exists no (congruent) triangulation of $R^{n}$ such that all one-faces of the simplices have the same length. Nevertheless given some accuracy we want to minimize the number of grid points per unit cube (on the average) since in that case one may expect that the number of iterations to find a completely labelled n-simplex is minimized. Since the class $A$ is very suitable for use in fixed point algorithms we restrict ourselves for the moment to $(\alpha, \beta)$-triangulations. The number of grid points per unit cube is on the average equal to one over the volume of $n$ ! simplices. Note that the latter is equal to $(\operatorname{det} A(\alpha, \beta))^{-1}$, where $A(\alpha, \beta)$ is the triangulation matrix of the $(\alpha, \beta)-$ triangulation. The accuracy of a triangulation corresponds to the total (squared) lengths of the one-faces of a simplex.

The diameter seems to be a too rough estimate for the accuracy. For example, let $\sigma\left(\mathrm{y}^{1}, \mathrm{y}^{2}, \mathrm{y}^{3}\right)$ be $\mathrm{a}$-triangle in $\mathrm{R}^{2}$ and let $\operatorname{diam} \sigma=|| \mathrm{y}^{1}-\mathrm{y}^{2}||$. If we move $y^{3}$ closer to the line segment $\left[y^{1}, y^{2}\right]$, the accuracy improves but the diameter remains the same. The total length of the one-faces decreases however, whereas also the volume of $\sigma$ decreases. If we move $y^{3}$ away from $\left[y^{1}, y^{2}\right]$ the accuracy becomes worser whereas the diameter does not change as long as the distance between $\mathrm{y}^{1}$ and $\mathrm{y}^{2}$ is more than the distance between $\mathrm{y}^{3}$ and $y^{1}$ and $y^{3}$ and $y^{2}$. Now the total length of the one-faces increases. The same reasoning can be given for $n \geq 3$. So, the sum of the squared lengths of the one-faces divided by the volume of a simplex seems to be a good measure to characterize the efficiency of a triangulation for use in a fixed point algorithm. In general we have the following definition. 
DEFINITION 5.5.1. For a triangulation $\mathrm{G}$ of an $\mathrm{m}$-dimensional convex subset $C$ of $R^{n}$, the sum of the squared edge lengths-Volume-measure (SV-measure), to be denoted by $S V(G)$, is defined by

$$
\operatorname{SV}(G)=\sup _{\sigma \in G}\left\{\left(\sum \operatorname{diam}^{2} \tau\right)^{\frac{1}{2}} /(\text { volume } \sigma)^{1 / n}\right\}
$$

where the sum is taken over all one-faces $\tau$ of $\sigma$.

The term of the numerator between brackets reflects the sum of the squared lengths of all one-faces of $\sigma$, whereas the term of the denominator is the volume of $\sigma$. By raising the first to the power a half and the latter to the power $\mathrm{n}^{-1}$, we obtain a measure being homogeneous of degree zero in the grid size. So, the SV-measure of a triangulation can be seen as a measure for the number of grid points per unit cube relatively to the accuracy of the triangulation. Obviously, if $\mathrm{n}=2$ the sv-measure is minimized for the equilateral triangulation. Before we calculate the optimal triangulation for $\mathrm{n} \geq 3$, we compute the SV-measure of an $(\alpha,-1)$ triangulation, to be denoted by SV $(\alpha)$. Observe that the SV-measure of an $\left(\alpha^{\prime}, \beta\right)$-triangulation is equal to

$$
\operatorname{SV}\left(\alpha^{\prime}, \beta\right)=\operatorname{SV}(\alpha)
$$

where $\alpha=\alpha \cdot /|\beta|$.

THEOREM 5.5.2.

$$
\operatorname{SV}(\alpha)=r_{n}\left(n^{2}-n-2 \alpha n+2 \alpha+2 \alpha^{2}\right)^{\frac{1}{2}} /\left\{(\alpha-n+1)(\alpha+1)^{n-1}\right\}^{1 / n}
$$

where $r_{n}=\{n(n+1)(n+2) / 12\}^{\frac{1}{2}}(n !)^{1 / n}$.

Further

$$
S V(K)=r_{n} \sqrt{2}
$$

PROOF. Since an $\alpha$-triangulation is regular and congruent, the SV-measure simplifies to

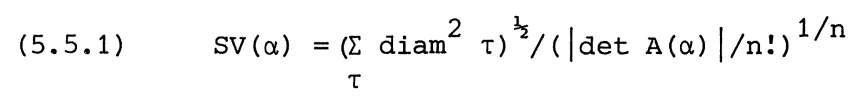


where the sum is taken over all one-faces of the standard simplex $s\left(y^{1}, \ldots, y^{n+1}\right)$ and where $A(\alpha)$ is the triangulation matrix of the $\alpha$-triangulation. From lemma 5.2 .6 we have that $\operatorname{det} A(\alpha)=(\alpha-n+1)(\alpha+1)^{n-1}$. Moreover, to calculate the numerator of the right side of (5.5.1) observe that

$$
\left\|y^{k}-y^{j}\right\|=|| y^{k-j+1} \| \quad 1 \leq j<k \leq n+1
$$

since $y_{i}^{j+1}=\alpha-(j-1)$ for $i \leq j$ and $y_{i}^{j+1}=-j$ for $i>j, j \in I_{n}$. Hence, the number of one-faces of $s\left(y^{1}, \ldots, y^{n+1}\right)$ having length $\left\|y^{k+1}\right\|$ is equal to $n-k+1, k \in I_{n}$. So the total squared lengths of the one-faces of $s_{n}$ is equal to

$$
\begin{aligned}
\sum_{\tau} \operatorname{diam}^{2} \tau & =\sum_{k=1}^{n}(n-k+1)\left\{k(\alpha-k+1)^{2}+(n-k) k^{2}\right\} \\
& =n(n+1)(n+2)\left(n^{2}-n-2 \alpha n+2 \alpha+2 \alpha^{2}\right) / 12 .
\end{aligned}
$$

Taking the above expressions together we get the desired result. The result for the $\mathrm{K}$ triangulation follows from the fact that (5.5.1) also holds for this triangulation.

COROLLARY. $5 \cdot 5 \cdot 3$.

$$
\lim _{\alpha \rightarrow \infty} \operatorname{SV}(\alpha)=\mathrm{SV}(\mathrm{K})
$$

THEOREM 5.5.4.

$$
\operatorname{SV}(n+\sqrt{n+1})=\min _{\alpha>n-1} \operatorname{SV}(\alpha)=r_{n}(n+1)^{1 / 2 n}
$$

PROOF. Let $\mathrm{C}(\alpha, \mathrm{n})$ be $\left(\mathrm{SV}(\alpha) / \mathrm{r}_{\mathrm{n}}\right)^{2}$. Then

$$
\begin{aligned}
& \frac{\operatorname{dSV}(\alpha)}{d \alpha}=\frac{1}{2} r_{n} C(\alpha, n)^{-\frac{1}{2}} \frac{d C(\alpha, n)}{d \alpha}= \\
& \quad D(\alpha, n)[(2 \alpha+1-n)(\alpha-n+1)(\alpha+1)- \\
& \left.\quad\left(n^{2}-n-2 \alpha n+2 \alpha^{2}+2 \alpha\right)\{\alpha+1+(\alpha-n+1)(n-1)\} / n\right],
\end{aligned}
$$


where $D(\alpha, n)=\{C(\alpha, n)\}^{-\frac{1}{2}} r_{n}(\alpha-n+1)^{-(n+2) / n}(\alpha+1)^{-(3 n-2) / n}$. Certainly, $\mathrm{D}(\alpha, \mathrm{n})$ is positive for $\alpha>\mathrm{n}-1, \mathrm{n}=1,2, \ldots$. Putting now $\operatorname{dSV}(\alpha) / d \alpha$ equal to zero we obtain

$$
\alpha^{2}-\alpha^{2} n+2 \alpha n^{2}-2 \alpha n-n^{3}+2 n^{2}-1=0
$$

which implies

$$
\alpha_{1,2}=n \pm \sqrt{n+1}
$$

Since $\alpha$ must be greater than $n-1$, it follows that $n+\sqrt{n+1}$ is the only feasible solution, yielding a minimum of $\mathrm{SV}(\alpha)$ as can easily be seen. Clearly, this minimum is less than $\mathrm{SV}(\mathrm{K})$ as follows from theorem 5.5.2. To calculate $S V(n+\sqrt{n+1})$ we obtain from lemma 5.5.2 that

$$
\begin{gathered}
\operatorname{SV}(n+\sqrt{n+1})=r_{n}\left\{n^{2}-n-2 n(n+\sqrt{n+1})+2(n+\sqrt{n+1})+2(n+\sqrt{n+1})^{2}\right\}^{\frac{1}{2}} / \\
\left\{(1+\sqrt{n+1})(n+1+\sqrt{n+1})^{n-1}\right\}^{1 / n} .
\end{gathered}
$$

After simple calculations we get that the numerator is equal to $\sqrt{n+1}(1+\sqrt{n+1}) r_{n}$ and the denominator to $(1+\sqrt{n+1})(\sqrt{n+1})(n-1) / n$. Notice that $n+2+2 \sqrt{n+1}=(1+\sqrt{n+1})^{2}$ and $n+1+\sqrt{n+1}=\sqrt{n+1}(1+\sqrt{n+1})$. Hence,

$$
S V(n+\sqrt{n+1})=r_{n}(n+1)^{1 / 2 n}
$$

We now give some interesting properties of the $\alpha^{*}$-triangulation where $\alpha^{*}=\mathrm{n}+\sqrt{\mathrm{n}+1}$.

THEOREM 5.5.5. The barycentre of a simplex of the $\alpha{ }^{*}$-triangulation has the same distance to each vertex of the simplex. Moreover, this distance is equal to $\{\mathrm{n}(\mathrm{n}+2)\}^{\frac{1}{2}}(1+\sqrt{\mathrm{n}+1}) / 2 \sqrt{3}$.

PROOF. Let $b$ be the barycenter of $s\left(y^{1}, \ldots, y^{n+1}\right)$. Since $b=\Sigma_{i=1}^{n+1} y^{i} /(n+1)$ it can easily. be seen that

$$
b_{j}=\left\{(n+1) \alpha^{*}-j \alpha^{*}-\frac{1}{2} n(n-1)-j+1\right\} /(n-1) \quad j=1, \ldots, n \text {. }
$$


The distance $a_{j}$ between $b$ and the vertex $y^{j+1}$ is equal to

$$
d_{j}=\left[\sum_{i=1}^{j}\left\{b_{i}-\alpha+j-1\right\}^{2}+\sum_{i=j+1}^{n}\left(b_{i}+j\right)^{2}\right]^{\frac{1}{2}} \quad j=0, \ldots, n .
$$

After simple calculations we obtain that $d_{j}$ is independent of $j$ for all $j$ and is equal to

$$
|| b-y^{1}||=\{n(n+2)\}^{\frac{1}{2}}(1+\sqrt{n+1}) / 2 \sqrt{3},
$$

which proves the theorem since the $\alpha^{*}$-triangulation is congruent.

The theorem says that the simplices of the optimal $\alpha$-triangulation are as "round" as possible.

THEOREM 5.5.6. Let $s\left(y^{1}, \ldots, y^{n+1}\right)$ be the standard simplex of the $\alpha^{*}$-triangulation. Then

(i) $\left\|y^{k+1}\right\|=\left\|y^{n-k+2}\right\| \quad k=1, \ldots, n$,

(ii) $\operatorname{mesh} A\left(\alpha^{*}\right)=|| y^{\frac{1}{2}(n+3)}||=\frac{1}{2}(1+\sqrt{n+1})(n+1)$,

n odd

$$
=|| y^{\frac{1}{2}(n+2)}||=|| y^{\frac{1}{2}(n+4)}||=\frac{1}{2}(1+\sqrt{n+1})\{n(n+2)\}^{\frac{1}{2}}, n \text { even. }
$$

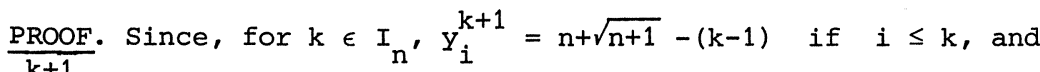
$y_{i}^{k+1}=-k$ if $i>k$, we have that

$$
\left.|| y^{k+1}\right|^{2}=k(n+\sqrt{n+1}-k+1)^{2}+(n-k) k^{2}
$$

and

$$
|| y^{n-k+2}||^{2}=(n-k+1)(\sqrt{n+1}+k)^{2}+(k-1)(n-k+1)^{2},
$$

which proves part a.

To prove part $b$, recall from the proof of lemma 5.5.2 that ||$y^{k}-y^{j}||=|| y^{k-j+1}||$ for $1 \leq j<k \leq n+1$. Therefore

$$
\operatorname{mesh} A\left(\alpha^{*}\right)=\operatorname{diam} s_{n}=\max _{k \in I_{n+1}}|| y^{k}||=
$$




$$
=\max _{k \in I_{n}}\left\{k(n+\sqrt{n+1}-k+1)^{2}+(n-k) k^{2}\right\}^{\frac{1}{2}},
$$

which also follows from theorem 5.2.5.

It is now easy to see that the maximum is attained for $k=\frac{1}{2}(n+3)$ if $n$ is odd and for $k=\frac{1}{2}(n+2)$ and $k=\frac{1}{2}(n+4)$ if $n$ is even.

Theorem 5.5.6 implies that the distance between $y^{n+1}$ and $y^{1}$ is equal to the distance between $y^{i}$ and $y^{i+1}, i=1, \ldots, n$. The $\alpha^{*}$-triangulation is the only triangulation in A having this property. Moreover, for the optimal $\alpha$-triangulation the distance between $y^{n+1}$ and $y^{i}$ is equal to the distance between $y^{1}$ and $y^{i+1}, i=2,3, \ldots, n$. Of course, these results apply to any simplex. In the following theorem due to Todd [private communication] let $a^{*}(i)$ be the $i$-th column of $A\left(\alpha^{*}\right), i \in I_{n}$, and let $a^{*}(n+1)=-\sum_{i=1}^{n} a^{*}(i)$, i.e. $a^{*}(n+1)=y^{1}-y^{n+1}=(-1-\sqrt{n+1})$ e. In the sequel we call the matrix

$$
\left[a^{*}(1) \quad \cdots \quad a^{*}(n) \quad a^{*}(n+1)\right]
$$

the extended $A\left(\alpha^{*}\right)$ matrix.

THEOREM 5.5.7. The angie between any two pair vectors $a^{*}(i)$ and $a^{*}(j)$ is the same, $i \neq j$. Moreover, this angle is equal to $\arccos n^{-1}$.

Finally, let $\tau_{i}$ be the facet of a simplex $\sigma\left(y^{1}, \ldots, y^{n+1}\right)$ of the $\alpha^{*}$-triangulation opposite the vertex $y^{i}, i=1, \ldots, n+1$.

THEOREM 5.5.8. The volume of $\tau_{i}$ is independent of $i, i \in I_{n+1}$.

The proof follows from the fact that in view of theorem 5.5 .6 all facets are congruent to each other.

Summarizing we have the following properties for the $\alpha^{*}$-triangulation:

1. the barycentre of a simplex has the same distance to all the vertices of the simplex

2. the columns of the (extended) $A\left(\alpha^{*}\right)$ matrix have the same length

3. the columns of the (extended) $A\left(\alpha^{*}\right)$ matrix make the same angle to each other

4. all facets have the same volume. 
Therefore, we can conclude that the $(n+\sqrt{n+1})$-triangulation is the most suitable regular triangulation for use in a fixed point algorithm. Note that this triangulation of $R^{n}$ is a straightforward generalization of the equilateral $(2+\sqrt{3})$-triangulation of $R^{2}$.

For $\mathrm{n}=3$ the standard simplex of the $(n+\sqrt{n+1})$-triangulation is drawn in figure 5.5.1. Note that $\rho\left(y^{1}, y^{2}\right)=\rho\left(y^{2}, y^{3}\right)=\rho\left(y^{3}, y^{4}\right)=\rho\left(y^{4}, y^{1}\right)=3 \sqrt{3}$ and $\rho\left(y^{1}, y^{3}\right)=\rho\left(y^{2}, y^{4}\right)=6$.

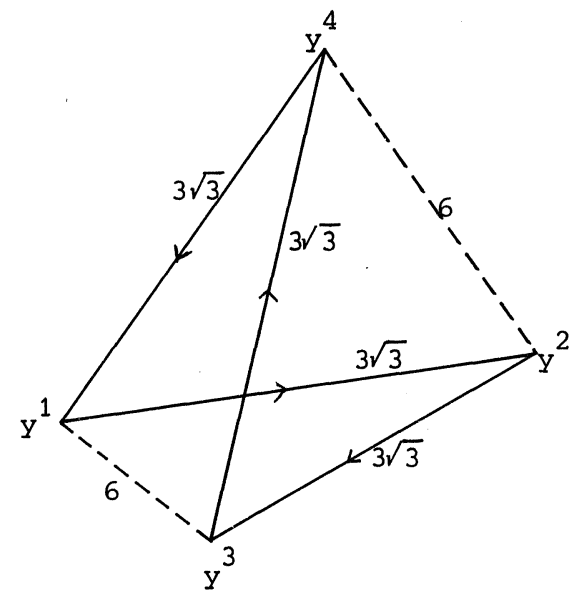

Figure 5.5.1. The standard simplex of the optimal $\alpha$-triangulation if $\mathrm{n}=3$.

A measure closely related to the SV-measure was independently developed by Todd [private communication]. This measure, called the diameter-volume measure and to be denoted by $D V(G)$, is based on the ratio of the diameter and the volume of a simplex.

DEFINITION 5.5.9. The diameter-volume measure of a triangulation $G$ is defined by

$$
\mathrm{DV}(\mathrm{G})=\sup _{\sigma \in \mathrm{G}}\left\{\text { diam } \sigma /(\text { volume } \sigma)^{1 / \mathrm{n}}\right\}
$$

For an $\alpha$-triangulation this measure simplifies to

$$
\operatorname{DV}(\alpha)=\operatorname{diam} s_{n} /(|\operatorname{det} A(\alpha)| / n !)^{1 / n}
$$


Note that this measure is also homogeneous of degree zero in the grid size. To calculate the optimal $\alpha$-triangulation according to this measure, observe that diam $s_{n}=\max _{j}\left\|y^{j}\right\|$ is not a differentiable function of $\alpha$. Therefore, diam $s_{n}$ is smoothened by setting it equal to

Hence,

$$
\operatorname{diam} s_{n} \approx \max _{0 \leq x \leq n}\left\{x(\alpha-x+1)^{2}+(n-x) x^{2}\right\}^{\frac{1}{2}}
$$

$$
\text { diam } s_{n} \approx \frac{1}{2}(\alpha+1)^{2} /(2+2 \alpha-n)^{\frac{1}{2}} \text {. }
$$

Recall that $\operatorname{det} A(\alpha)=(\alpha-n+1)(\alpha+1)^{n-1}$.

Todd stated that $\mathrm{DV}(\alpha)$ for $\alpha>n-1$ was minimized by $\alpha=\frac{1}{2}(3+\sqrt{3}) \mathrm{n}-1$.

However, he made an error in his calculations. In fact the DV-measure is minimized for $\alpha^{*}=\mathrm{n}+\sqrt{\mathrm{n}+1}$, yielding the same optimal $\alpha$-triangulation as for the SV-measure as could be expected. However, to compare in general the efficiency of triangulations for use in fixed point algorithms, it seems more naturai to use the total squared length of the one-faces of a simplex rather that its diameter (see also Todd and Acar [1978, page 3]).

5.6. COMPARISON OF SEVERAL TRIANGULATIONS OF $R^{\mathrm{n}}$.

To compare the $\alpha^{*}$-triangulation with the $\mathrm{K}$ - and $\mathrm{H}$ triangulations, we shall calculate both their average directional density and their SV-measure. Together corollary 5.4.5 and theorem 5.5.6 imply

$$
\begin{aligned}
N\left(A^{*} K\right) & =\{n(n+1) / 8\}^{\frac{1}{2}}(n+1) g_{n} \quad \text { if } n \text { is odd } \\
& =\{n(n+1) / 8\}^{\frac{1}{2}}\{n(n+2)\}^{\frac{1}{2}} g_{n} \quad \text { if } n \text { is even, }
\end{aligned}
$$

where $A^{*}$ is the matrix of the optimal $\alpha^{*}$-triangulation with grid size such that mesh $\left(A^{*} K\right)=\sqrt{n}$. Consequently, we have (cf. Corollary 5.4.8).

COROLLARY 5.6.1. The average directional density of the optimal $\alpha$-triangulation having mesh equal to $\sqrt{n}$ is of the order $g_{n} n^{2} / \sqrt{8}$ for $n$ large enough. 
Recall from theorem 5.4.3 and corollary 5.4.4 that

and

$$
N(K)=\{n+n(n-1) / \sqrt{2}\}_{n}
$$

$$
N(H)=\left\{\sum_{j=1}^{n}(n-j+1) \sqrt{j}\right\} g_{n} .
$$

Table 5.6.1. gives $N(G) / g_{n}$ for several values of $n$ for the $H-, K-$ and $\mathrm{A}^{*} \mathrm{~K}$ triangulations.

From theorem 5.5.4 we know that

$$
\operatorname{SV}\left(A^{*} K\right)=\operatorname{SV}\left(\alpha^{*}\right)=r_{n}(n+1)^{1 / 2 n}
$$

and from theorem 5.5.2

$$
\mathrm{SV}(\mathrm{K})=\mathrm{r}_{\mathrm{n}} \sqrt{2}
$$

Finally, to calculate SV(H) we have to compute

$$
\text { (5.6.1) } \max _{\sigma \in \mathrm{H}}\left(\Sigma_{\tau} \operatorname{diam}^{2} \tau\right) \text {, }
$$

where the sum is taken over all one-faces of $\sigma$, since the H-triangulation is not congruent. Note that volume $\sigma=1$ for any simplex $\sigma$. Since $H$ is a regular triangulation, we can restrict ourselves to simplices $\sigma(\underline{0}, \pi)$. It is then easy to see that the maximum in $(5.6 .1)$ is attained for

$$
\begin{aligned}
\pi & =(1,3,5, \ldots, \mathrm{n}, 2,4, \ldots, \mathrm{n}-1) \quad \text { if } \mathrm{n} \text { is odd } \\
& =(2,4,6, \ldots, \mathrm{n}, 1,3, \ldots, \mathrm{n}-1) \quad \text { if } \mathrm{n} \text { is even. }
\end{aligned}
$$

Hence, the sum of the squared lengths of the edges of this simplex is equal to $\left(n^{3}+2 n^{2}-n+2\right) / 4$ if $n$ is odd and to $\left(n^{3}+2 n^{2}\right) / 4$ if $n$ is even. So, the SV-measure of the H-triangulation is equal to

$$
\begin{aligned}
S V(H) & =r_{n}\left\{3\left(n^{3}+2 n^{2}-n+2\right) / n(n+1)(n+2)\right\}^{\frac{1}{2}} & & \text { if } n \text { is odd } \\
& =r_{n}\{3 n /(n+1)\}^{\frac{1}{2}} & & \text { if } n \text { is even. }
\end{aligned}
$$


Observe that

$$
\lim _{n \rightarrow \infty} S V(H)=r_{n} \sqrt{3}, \lim _{n \rightarrow \infty} S V(K)=r_{n} \sqrt{2} \text { and } \underset{n \rightarrow \infty}{\lim } \operatorname{SV}\left(\alpha^{*}\right)=r_{n}
$$

Table 5.6.2 gives $\mathrm{SV}(\mathrm{G}) / \mathrm{r}_{\mathrm{n}}$ for the same values of $\mathrm{n}$ for the $\mathrm{H}-, \mathrm{K}-$, and $\alpha^{*}$-triangulations.

\begin{tabular}{lc|cccccccccccc}
$\mathrm{G}$ & $\mathrm{n}$ & 1 & 2 & 3 & 4 & 5 & 9 & 15 & 20 & 30 & 50 & 100 & $\mathrm{n}$ \\
\hline $\mathrm{H}$ & 1 & 3.4 & 7.6 & 13.7 & 22.1 & 82.0 & 269.4 & 534 & 1420 & 4942 & $27316 \sim_{4 \mathrm{n}}^{5 / 2} / 15$ \\
$\mathrm{~K}$ & 1 & 3.4 & 7.2 & 12.5 & 19.1 & 59.9 & 163.5 & 289 & 645 & 1782 & 7100 & $\mathrm{nn}^{2} / \sqrt{2}$ \\
$\mathrm{~A}^{*} \mathrm{~K}$ & 1 & 2.4 & 4.9 & 7.7 & 11.6 & 33.5 & 87.6 & 152 & 334 & 910 & $3553 \sim^{2} / \sqrt{8}$
\end{tabular}

Table 5.6.1. The a.d.d. of the $\mathrm{H}_{-}, \mathrm{K}-$, and $\mathrm{A}^{*} \mathrm{~K}$ triangulations (mesh is equal to $\sqrt{n}$ ).

\begin{tabular}{|c|c|c|c|c|c|c|c|c|c|c|c|}
\hline $\mathrm{G} \quad \mathrm{n}$ & 1 & 2 & 3 & 4 & 5 & 9 & 15 & 20 & 30 & 50 & 100 \\
\hline $\mathrm{H}$ & 1.41 & 1.41 & 1.48 & 1.55 & 1.57 & 1.64 & 1.67 & 1.69 & 1.70 & 1.71 & $1.72 \sim 1.73$ \\
\hline $\mathrm{K}$ & 1.41 & . & $\cdot$. & & . . & . 1 & .41 & . & . & . & . . 1.41 \\
\hline$A^{*} K$ & 1.41 & 1.32 & 1.26 & 1.22 & 1.20 & 1.14 & 1.10 & 1.08 & 1.06 & 1.04 & $1.02 \sim 1$ \\
\hline
\end{tabular}

Table 5.6.2. The SV-measure of the $\mathrm{H}-, \mathrm{K}-$, and $\mathrm{A}^{*} \mathrm{~K}$ triangulations.

Both for the $\mathrm{K}$ - and $\mathrm{A}^{*} \mathrm{~K}$ triangulation the basic algorithm of Van der Laan and Talman was applied to a ten-dimensional variant of a problem considered by Kellog, $\mathrm{Li}$ and Yorke [1976]. In table 5.6.3 the number of iterations is given for various starting points. For the $\mathrm{K}$ triangulation the grid size was equal to .05 whereas the $A^{*} \mathrm{~K}$ triangulation was normalized such that its mesh was equal to $.05 \sqrt{10}$. The accuracy of the approximate fixed point is of the order of $10^{-4}$. 


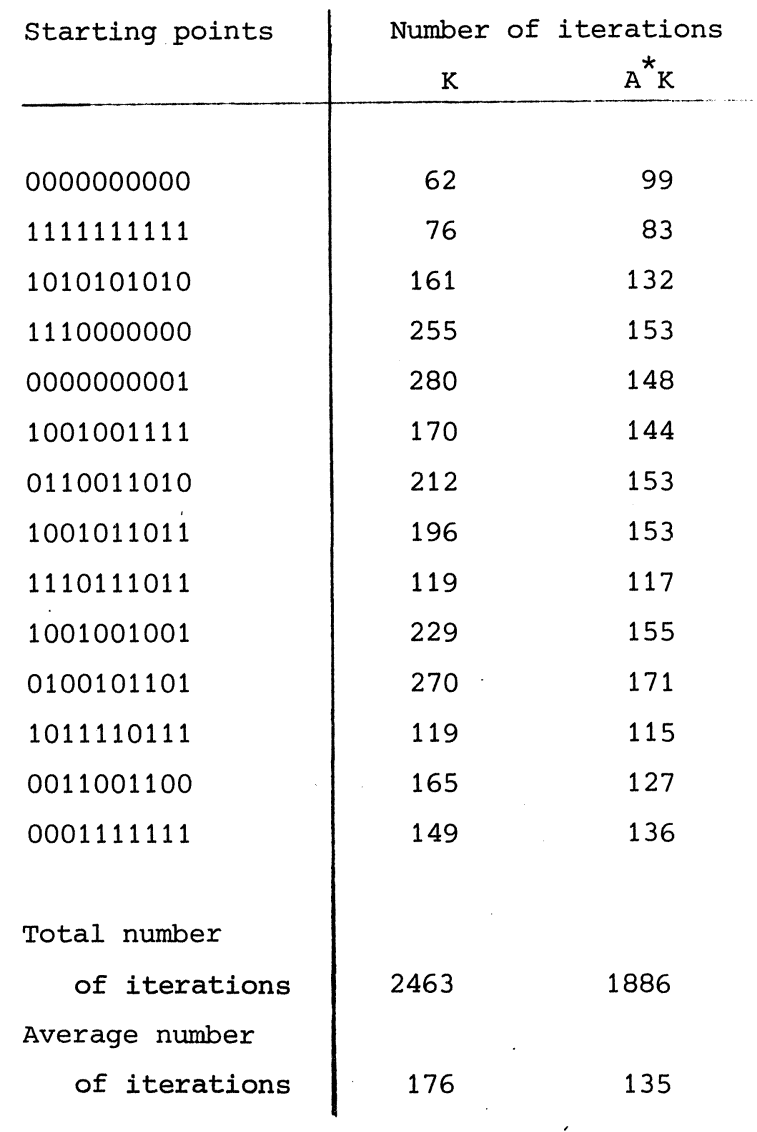

Table 5.6.3. Comparison of the $\mathrm{K}-$ and $\mathrm{A}^{*} \mathrm{~K}$ triangulations.

As appears from the table the version with the $\alpha^{*}$-triangulation performs considerably better than the (usually taken) $\mathrm{K}$ triangulation. Observe that the $\mathrm{K}$ triangulation is very appropriate for the first two starting points because the path generated by the algorithm is along the direction e respectively -e. However, for many other starting points the algorithm needs for the $\mathrm{K}$ triangulation much more iterations than for the $\mathrm{A}^{*} \mathrm{~K}$ triangulation, since for the first one a path is generated in a direction which is inappropriate for this triangulation. Moreover, since the $A{ }^{*} K$ triangulation has as "round" simplices as possible, the number of iterations does not vary so much as for the $\mathrm{K}$ triangulation. Therefore, one can expect that the average number of iterations for the $A^{*} \mathrm{~K}$ triangulation is lower than for the $\mathrm{K}$ triangulation (see also the computational results in chapter 7 ). 
5.7. A GOOD TRIANGULATION OF THE AFFINE HULL OF $S^{n}$.

In this section a new triangulation of the affine hull $U^{n}$ of $S^{n}$ is proposed. We shall prove that this triangulation has the same SV-measure as the optimal $\alpha$-triangulation of $R^{n}$. Also we will compare its SV-measure with the SV-measure of the standard triangulation of $\mathrm{s}^{\mathrm{n}}$.

To motivate the new triangulation, observe that under the standard labelling rule on $U^{n}$ with the condition on the function $f$ as stated by Van der Laan and Talman [1980c] and Van der Laan [1980], there is an incentive to decrease the $i-t h$ component if label $i$ is found, i.e. to move in the direction where label $i$ is missing, $i \in I_{n+1}$. Then for the standard triangulation of $U^{n}$ the $(i+1)-t h$ component is increased with the same amount whereas the other $n-1$ components remain the same ( $i+1=1$ if $i=n+1)$. However, there is no reason to increase only a special component quite a lot and the other components not at all. On the contrary, it seems to be natural to increase all other components (except the i-th one) with the same amount, which then must be equal to a fraction $\mathrm{n}^{-1}$ of the amount with which the i-th component is decreased. Therefore we propose the following triangulation, to be called the $U$ triangulation.

DEFINITION 5.7.1. The $U$ triangulation of $U^{n}$ with grid size $m^{-1}(m>0)$ is the collection of simplices $\sigma\left(y^{1}, \pi\right)$ with vertices $y^{1}, \ldots, y^{n+1}$ such that

(i) $y^{1}=(n+1)^{-1} e+m^{-1} \sum_{j=1}^{n} \lambda_{j} u(j)$ for integers $\lambda_{j^{\prime}} j \in I_{n}$,

(ii) $\pi=\left(\pi_{1}, \ldots, \pi_{n}\right)$ is a permutation of the elements of $I_{n}$,

(iii) $y^{i+1}=y^{i}+m^{-1} u\left(\pi_{i}\right), \quad i=1, \ldots, n$,

where $u(j)$ is the $j-t h$ column of the $(n+1)$ xn matrix $U$ defined by

$\mathrm{U}=\left[\begin{array}{rrrrr}-\mathrm{n} & 1 & \cdot & \cdot & 1 \\ 1 & -\mathrm{n} & & & \cdot \\ \cdot & \cdot & \cdot & & \cdot \\ \cdot & & \cdot & \cdot & 1 \\ 1 & \cdot & \cdot & 1 & -\mathrm{n} \\ 1 & \cdot & \cdot & \cdot & 1\end{array}\right]$. 
Moreover, let $u(n+1)=-\sum_{j=1}^{n} u(j)$, i.e.

$$
u(n+1)=(1, \ldots, 1,-n)^{\top} \text {. }
$$

Indeed, if label $\mathrm{n}+1$ is found, we decrease the last component with $\mathrm{nm}^{-1}$ and increase the other components with $\mathrm{m}^{-1}$.

THEOREM 5.7 .2 .

$$
\operatorname{SV}(U)=r_{n}(n+1)^{1 / 2 n}
$$

PROOF. Since the SV-measure is homogeneous, SV(U) is independent of the grid size. Define now the $(n+1) x(n+1)$ matrix $\bar{D}$ by (cf. Todd [1978a])

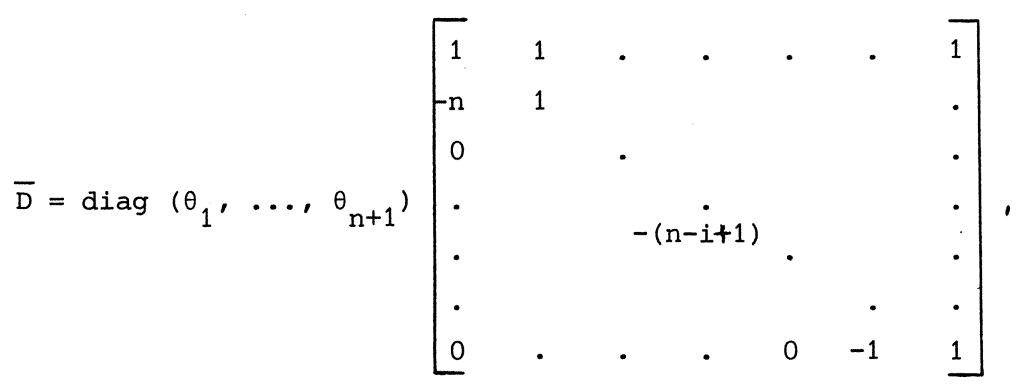

where diag $\left(\theta_{1}, \ldots, \theta_{n+1}\right)$ is the $(n+1)$-diagonal matrix with $i-t h$ element equal to

$$
\theta_{i}=(n+1)^{-\frac{1}{2}} \quad i=1
$$

and

$$
\theta_{i}=\{(n-i+2)(n-i+3)\}^{-\frac{1}{2}} \quad i=2, \ldots, n+1 \text {. }
$$

Let $D$ be the $n x(n+1)$ matrix consisting of the last $n$ rows of $\bar{D}$, then $D$ maps $U^{n}$ into $R^{n}$ orthogonally so that the distance between any two points under this mapping is preserved. Note that the matrix $Q$ defined in section 2.6 is not orthogonal. Therefore, the matrix D makes it possible to calculate the volume of a simplex of the (congruent) $U$ triangulation since the volume of such a simplex is now equal to the volume of a simplex of the DTK triangulation of $R^{n}$. But the latter is equal to $\mid$ det $D T \mid / n !$. Clearly, the nxn matrix DT is uppertriangular with i-th diagonal element equal to 


$$
(n+1)\{(n+1-i) /(n+2-i)\}^{\frac{1}{2}} \quad i=1, \ldots, n
$$

so that det $D T=(n+1)^{n-\frac{1}{2}}$. Moreover, the sum of the squared lengths of the edges of a simplex of the $U$ triangulation is equal to

$$
\sum_{k=1}^{n}(n+1-k)\left\{k(n+1-k)^{2}+(n+1-k) k^{2}\right\}=n(n+1)^{3}(n+2) / 12 .
$$

Therefore,

$$
\operatorname{SV}(U)=\left\{n(n+1)^{3}(n+2) / 12\right\}^{\frac{1}{2}} /\left\{(n+1)^{n-\frac{1}{2}} / n !\right\}^{1 / n}=r_{n}(n+1)^{1 / 2 n} .
$$

From this theorem it follows that the U-triangulation of $U^{\mathrm{n}}$ has the same SV-measure as the $\alpha^{*}$-triangulation of $\mathrm{R}^{\mathrm{n}}$. Moreover, it is easy to prove that the triangulation has the same nice properties as stated in section 5.4 for the $\alpha^{*}$-triangulation. Note, however, that the simplices of the $\mathrm{U}$ triangulation of $\mathrm{U}^{\mathrm{n}}$ meeting $\mathrm{S}^{\mathrm{n}}$ do not all lie in $\mathrm{S}^{\mathrm{n}}$. So, to approximate a fixed point of an u.s.c. mapping from $S^{\mathrm{n}}$ to the set of nonempty, convex subsets of $s^{n}$, it is necessary to extend the mapping to an u.s.c. mapping $\bar{\phi}$ on $\mathrm{U}^{\mathrm{n}}$ such that $\bar{\phi}(\mathrm{x})=\phi(\mathrm{x})$ if $\mathrm{x} \in \mathrm{S}^{\mathrm{n}}$, and $\mathrm{x}^{*} \in \bar{\phi}\left(\mathrm{x}^{*}\right)$ implies $\mathrm{x}^{*} \in \mathrm{S}^{\mathrm{n}}$. This can be achieved by mapping all points outside $S^{\mathrm{n}}$ (and on the boundary of $s^{n}$ ) to the point $e(n+1)^{-1}$ or to the starting point $y$. Next we compute the SV-measure of the $Q$ triangulation of $s^{n}$.

THEOREM $5 \cdot 7 \cdot 3$.

$$
\begin{aligned}
\operatorname{SV}(Q) & =r_{n} \sqrt{3}(n+1)^{-1 / 2 n} \quad \text { if } n \text { is even } \\
& =r_{n} \sqrt{3}\left\{\left(n^{3}+3 n^{2}-n+5\right) /\left(n^{3}+3 n^{2}+2 n\right)\right\}^{\frac{1}{2}}(n+1)^{-1 / 2 n} \text { if } n \text { is odd. }
\end{aligned}
$$

PROOF. Let $D$ be again the $\mathrm{nx}(\mathrm{n}+1)$ matrix defined in the proof of theorem 5.7 .2 ; then the nxn matrix $\mathrm{DQ}$ is lower-triangular with i-th diagonal element equal to

$$
\{(n-i+2) /(n-i+1)\}^{\frac{1}{2}} \quad i=1, \ldots, n \text {. }
$$

Hence, $\operatorname{det} \mathrm{DQ}=(\mathrm{n}+1)^{\frac{1}{2}}$, i.e. the volume of any simplex of the standard triangulation is equal to $(n+1)^{\frac{1}{2}} / n$ !. Furthermore, it can be rather easily 
seen that the maximum of the sum of the squared lengths of the edges of the simplices is attained for the simplices $\sigma\left(y^{1}, \pi\right)$ having $\pi$ equal to

$$
\begin{aligned}
& \pi=(2,4, \ldots, n, 1,3, \ldots, n-1) \text { if } n \text { is even } \\
& =(2 \mathrm{k}-1,2 \mathrm{k}+1,2 \mathrm{k}-3,2 \mathrm{k}+3, \ldots, 1, \mathrm{n}, 2 \mathrm{k}, 2 \mathrm{k}-2,2 \mathrm{k}+2, \ldots, 2, \mathrm{n}-1) \\
& \text { if } \mathrm{n}=4 \mathrm{k}-1, \mathrm{k}=1,2, \ldots \\
& =(2 \mathrm{k}-1,2 \mathrm{k}+1,2 \mathrm{k}-3, \ldots, \mathrm{n}, 1,2 \mathrm{k}, 2 \mathrm{k}-2,2 \mathrm{k}+2, \ldots, \mathrm{n}-1,2) \\
& \text { if } \mathrm{n}=4 \mathrm{k}-3, \mathrm{k}=1,2, \ldots
\end{aligned}
$$

For these simplices we have that the sum of the squared edge-lengths is equal to $\frac{1}{4}\left(n^{3}+3 n^{2}+2 n\right)$ if $n$ is even and to $\frac{1}{4}\left(n^{3}+3 n^{2}-n+5\right)$ if $n$ is odd. Consequently,

$$
\begin{aligned}
\operatorname{SV}(Q)= & \left\{\frac{1}{4}\left(n^{3}+3 n^{2}+2 n\right)\right\}^{\frac{1}{2}} /\left\{(n+1)^{\frac{1}{2}} / n !\right\}^{1 / n}=r_{n} \sqrt{3}(n+1)^{-1 / 2 n} \\
= & \left\{\frac{1}{4}\left(n^{3}+3 n^{2}-n+5\right)\right\}^{\frac{1}{2}} /\left\{(n+1)^{\frac{1}{2}} / n !\right\}^{1 / n}= \\
& r_{n} \sqrt{3}\left\{\left(n^{3}+3 n^{2}-n+5\right) /\left(n^{3}+3 n^{2}+2 n\right)\right\}^{\frac{1}{2}}(n+1)^{-1 / 2 n} \text { if even } n \text { is odd. }
\end{aligned}
$$

From the theorems 5.7.2 and 5.7.3 we can draw the conclusion that

$$
\lim _{n \rightarrow \infty} S V(U)=r_{n} \text { and } \lim _{n \rightarrow \infty} S V(Q)=r_{n} \sqrt{3}
$$

which agrees with the limits for the $\alpha^{*}$-triangulation and the $\mathrm{H}$ triangulation of $\mathrm{R}^{\mathrm{n}}$ respectively.

Note, however, that for $\mathrm{n}=(1$ and $) 2$ both triangulations have the same $\mathrm{SV}$-measure. For $\mathrm{n}=2$ equilateral triangles are generated. But even in this case the $U$ triangulation will converge faster than the standard triangulation since the first one is more natural as argued above. In table 5.7.1 $\mathrm{SV}(\mathrm{G}) / \mathrm{r}_{\mathrm{n}}$ is given for several values of $\mathrm{n}$ for the Q- and U triangulations. 


\begin{tabular}{c|ccccccccc}
$\mathrm{G}^{\mathrm{n}}$ & 1 & 2 & 3 & 4 & 10 & 20 & 50 & 100 & $\mathrm{n}$ \\
\hline $\mathrm{Q}$ & 1.41 & 1.32 & 1.33 & 1.42 & 1.50 & 1.60 & 1.67 & 1.70 & $\sim 1.73$ \\
$\mathrm{U}$ & 1.41 & 1.32 & 1.26 & 1.22 & 1.14 & 1.08 & 1.04 & 1.02 & $\sim 1$
\end{tabular}

Table 5.7.1. The SV-measure of the $Q^{-}$and $U$ triangulations.

The basic algorithm of Van der Laan and Talman was applied to the three economic problems of Scarf [1973] for both the Q-and U triangulation to compare them. As could be expected, the $\mathrm{u}$ triangulation performs much better than the $\mathrm{Q}$ triangulation. About the same results were obtained for the homotopy-algorithm of Van der Laan and Talman. Todd [private communication] compared also both triangulations and had the same experience. Finally, in table 5.7.2 the computational experience is given. All runs were done with and without acceleration. In the latter case the factor $f$ of incrementation was always equal to 2 . In the case of acceleration the new grid size was set equal to the maximal absolute - difference between the components of the last and the previous approximation unless this number was larger than a half of the previous grid size. Then the grid size was halfed. The accuracy of the last approximate fixed point was less than $10^{-10}$.

\begin{tabular}{ll|rrrrrr} 
& & \multicolumn{2}{|c}{$\mathrm{n}=4$} & \multicolumn{2}{c}{$\mathrm{n}=7$} & \multicolumn{2}{c}{$\mathrm{n}=9$} \\
& & acc. & $\mathrm{f}=2$ & acc. & $\mathrm{f}=2$ & acc. & $\mathrm{f}=2$ \\
\hline $\begin{array}{l}\text { Homotopy } \\
\text { algorithm }\end{array}$ & $\mathrm{Q}$ & 68 & 108 & 144 & 199 & 155 & 217 \\
& & 59 & 102 & 93 & 166 & 146 & 210 \\
Basic & $\mathrm{V}$ & 63 & 85 & 127 & 197 & 205 & 255 \\
algorithm & $\mathrm{U}$ & 50 & 66 & 121 & 182 & 124 & 188
\end{tabular}

Table 5.7.2. Computational results. 
CHAPTER 6

VARIABLE DIMENSION FIXED POINT ALGORITHM ON THE PRODUCT SPACE S OF UNIT SIMPLICES

6.1. INTRODUCTION .

Let $s$ be the product space of $N$ unit simplices $s^{m_{j}}, j=1, \ldots, N$. Observe that $S$ is an $M$-dimensional subset of $R^{M+N}$, where $M$ is equal to $\sum_{j=1}^{N} m_{j}$. In this chapter we develop a variable dimension restart algorithm to approximate a fixed point of a continuous function $f$ (or an u.s.c. mapping) from $S$ into itself. One possible approach is to consider $S$ as a subset of the set $S(N)=\left\{x \in R_{+}^{M+N} \mid \Sigma_{i} x_{i}=N\right\}$. By extending then the function (or mapping) to a mapping $\bar{\phi}$ from $s(N)$ into itself in the same way as described in chapter 3, the algorithms discussed in chapter 4 can be directly applied. Clearly, the disadvantage of doing so is that the dimension of the problem is increased with $\mathrm{N}-1$. Moreover, a completely labelled simplex of $\mathrm{S}(\mathrm{N})$ contains at least $\mathrm{N}-1$ artificially labelled vertices which affects the accuracy. Garcia, Lemke' and Lüthi [1973] developed in asimilar way an algorithm which is very close to Kuhn's variable dimension algorithm on $\mathrm{s}^{\mathrm{n}}$.

Another way to compute fixed points on $S$ is to change both the triangulation matrix and labelling rule of chapter 4 in such a way that we can apply the algorithms, discussed in chapter 4 for $s^{n}$, to $s$. Since $\operatorname{dim} S=M$, we need an $(M+N) \times M$ triangulation matrix $P$ (say), and $M+1$ labels in case of integer labelling. Note that the number of variables is equal to $\mathrm{M}+\mathrm{N}$. We can prove that a regular PK triangulation of $\mathrm{S}$ is obtained if the matrix $P$ is a block matrix defined by

$$
\mathrm{P}=\left[\begin{array}{lllll}
\mathrm{P}_{1} & 0 & \cdot & \cdot & 0 \\
0 & \mathrm{P}_{2} & & & \cdot \\
\cdot & & \cdot & & \cdot \\
\cdot & & & \cdot & 0 \\
0 & \cdot & \cdot & 0 & \mathrm{P}_{N}
\end{array}\right]
$$


where $P_{j}$ is the standard triangulation matrix of $s^{m} j, j=1, \ldots, N$ (see chapter 2), i.e. the PK triangulation of $\mathrm{S}$ having grid size $\mathrm{m}^{-1}(\mathrm{~m}>0)$ is the set of simplices $\sigma\left(y^{1}, \pi\right)$ with vertices $y^{1}, \ldots, y^{M+1}$ such that

(i) the components of $\mathrm{y}^{1}$ are a multiple of $\mathrm{m}^{-1}$,

(ii) $\pi$ is a permutation of the elements of $I_{M^{\prime}}$

(iii) $y^{i+1}=y^{i}+m^{-1} p\left(\pi_{i}\right) \quad i=1, \ldots, M$.

Concerning the labelling rule, however, we encounter some difficulties.

Recall that the number of variables is $\mathrm{M}+\mathrm{N}$ whereas we can only assign $\mathrm{M}+1$ labels in case of integer labelling. Thus (if $N>1$ ) we have to combine (or delete) some components of the $(M+N)$-vector $f(x)-x$ to determine the label of the point $x$. This can be done by deleting for $j=1, \ldots, N$ the $\left(\Sigma_{i=1}^{j}\left(m_{i}+1\right)\right)-t h$ component of $f(x)-x$ and assign label $M+1$ to $x$ if the remaining components are all positive. Otherwise, the standard labelling rule is used. However, this seems not very natural since some components are "discriminated". In case of vector labelling we have the same difficulties. In that case we can not assign the vector $-f(x)+x+e$ to the grid point $x$ since the sytem of linear equations consists of only $M+1$ columns (unknowns). Again we can delete N-1 rows without affecting the system but it seems not to be very natural.

To avoid these problems we introduce in this chapter an algorithm based on ideas of Van der Laan and Talman's basic algorithm on $S^{\mathrm{n}}$ as described in section 4.5. Therefore we need a special triangulation of $\mathrm{S}$ which will be defined in relation to an arbitrarily chosen starting point y. To each point $\mathrm{x} \in \mathrm{S}$ we assign in case of integer labelling an integer of the set $I_{M+N}$ and in case of vector labelling the $(M+N)$-vector $-f(x)+x+e$. Starting with the zero-dimension simplex $\{y\}$ the algorithm generates by pivot and replacement steps a sequence of adjacent simplices of the triangulation of variable dimension until a simplex is found which yields an approximate fixed point. In general, if a new label is found (or a unit vector is eliminated), the dimension of the current simplex is increased. Sometimes the dimension is decreased. We will prove that an approximate fixed point is found if some special set of labels is found (or if a special set of unit vectors has been eliminated). In total there are $\mathrm{N}$ of such sets. Moreover we will show that if $M+1$ different labels have been found, i.e. 
if all vertices of a full-dimensional simplex of $\mathrm{S}$ are differently labelled, the labelset contains at least one of these sets. We also prove that the algorithm terminates within a finite number of iterations with such a set. It will appear that the algorithm can stop with a lowerdimensional simplex without affecting the accuracy of the approximation. In section 2 the triangulation of $\mathrm{S}$ is introduced. For integer labelling section 3 gives the (proper) labelling rule and proves that the algorithm's terminal simplex provides an approximate fixed point. Section 4 presents the algorithm. In the same section the convergence is proved. The extension of the algorithm for vector labelling is described in section 5. Finally, computational experience is given in section 6 . In that section we approximate equilibrium strategies of the noncooperative $\mathrm{N}$ person game discussed in section 3.2 .

This chapter is based on the work of Van der Laan and Talman [1978a].

\subsection{TRIANGULATION OF $\mathrm{s}$.}

Roughly speaking, the triangulation of $S$ is obtained by first taking in a proper way the product of the (standard) triangulations of $s^{m_{j}}$ for given grid sizes $d_{j}^{-1}$ ( $d_{j}$ being a positive integer), $j=1, \ldots, N$. This product triangulation produces cells of the form $\sigma_{1} \times \sigma_{2} \times \ldots \sigma_{N}$, where $\sigma_{j}$ is an $m_{j}$-simplex of the triangulation of $s^{m_{j}}$. Note that these cells are full-dimensional . Clearly, each cell has $\pi_{j=1}^{N}\left(m_{j}+1\right)$ vertices. These cells are illustrated in figure 6.2.1 a for $\mathrm{N}=2, \mathrm{~m}_{1}=\mathrm{m}_{2}=1$, $\mathrm{d}_{1}=\mathrm{d}_{2}=4$, and in figure 6.2.1b for $\mathrm{N}=2, \mathrm{~m}_{1}=2, \mathrm{~m}_{2}=1$ and $\mathrm{d}_{1}=\mathrm{d}_{2}=2$. In these figures $u(i, j)$ is the $(M+N)$-vector having ones on the $i-t h$ and $\left(m_{1}+j+1\right)-t h$ places and zeroes elsewhere. Recall that $M=\Sigma_{j=1}^{N} m_{j}$. Each cell is now triangulated with respect to the arbitrarily chosen starting point into $M ! / \pi_{j=1}^{N} m_{j}$ : simplices in such a way that the collection of all these simplices constitutes a triangulation of S. Basically, however, each cell is triangulated in the same way. For example the squares in figure 6.2.1a are triangulated by taking one of the diagonals as is illustrated in figure 6.2.2a, whereas the cells in figure $6.2 .1 \mathrm{~b}$ are triangulated by subdividing the (three) facets being a square into two triangles. This is illustrated in figure $6.2 .2 \mathrm{~b}$. 


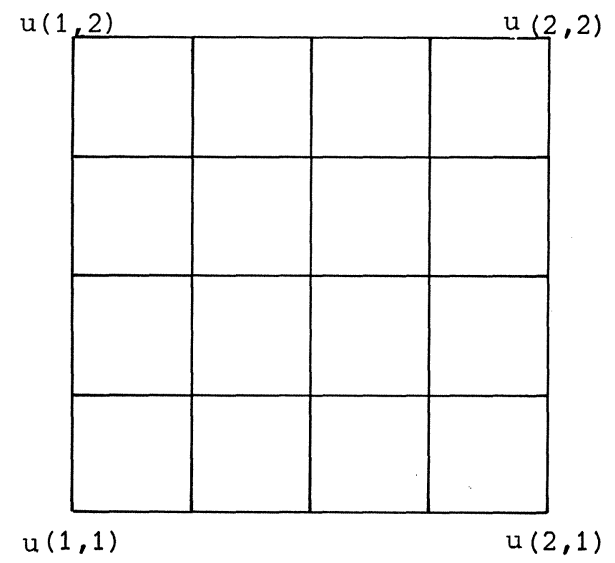

Figure 6.2.1a. Product triangulation of $S$ if $\mathrm{N}=2, \mathrm{~m}_{1}=\mathrm{m}_{2}=1, \mathrm{~d}_{1}=\mathrm{d}_{2}=4$.

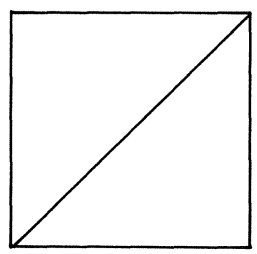

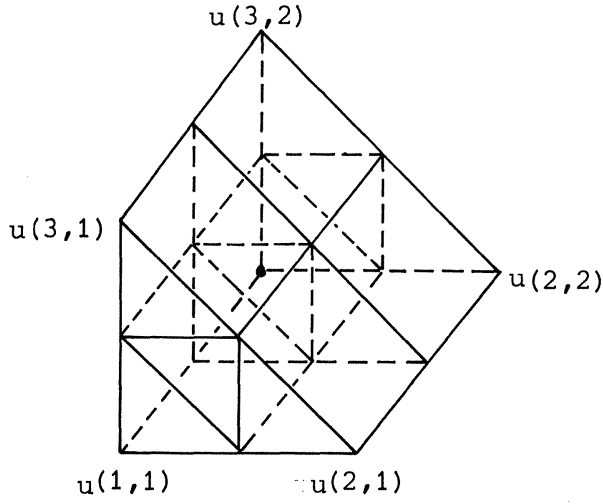

Figure 6.2.1b. Product triangulation of $\mathrm{S}$ if $\mathrm{N}=2, \mathrm{~m}_{1}=2, \mathrm{~m}_{2}=1, \mathrm{~d}_{1}=\mathrm{d}_{2}=2$.

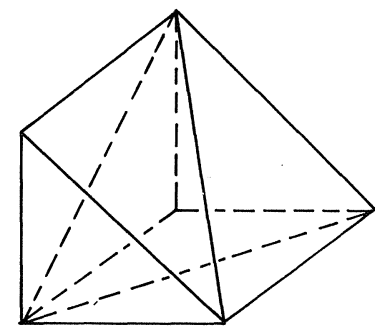

Figure 6.2.2b. Triangulation of a cell if $\mathrm{N}=2, \mathrm{~m}_{1}=2, \mathrm{~m}_{2}=1$.

$$
\text { , } 1 \text {, } 2
$$

Figure 6.2.2 a. Triangulation of a cell if $\mathrm{N}=2, \mathrm{~m}_{1}=\mathrm{m}_{2}=1$.

The triangulation needed in the algorithm will now be described in full detail without reference to the product triangulation. First we define the set of grid points of $s$. Let $d_{1}, \ldots, d_{N}$ be $N$ positive integers and let $s^{m_{j}}\left(d_{j}\right)$ be the set of grid points of the standard triangulation of $s^{m_{j}}$ with grid size $d_{j}^{-1}$, i.e. $s^{m_{j}}\left(d_{j}\right)$ is the set of points $x_{j} \in s^{m_{j}}$ such that each component of $x_{j}$ is a nonnegative multiple of $d_{j}^{-1}, j=1, \ldots, N$. Let $d=\left(d_{1}, \ldots, d_{n}\right)$, then the set $s(d)$ of grid points of $s$ is the product of the sets $s^{m_{j}}\left(d_{j}\right), j_{m_{j}} \in I_{N}$, i.e. $y=\left(y_{1}^{\top}, \ldots, y_{N}^{\top}\right)^{\top}$ is an element of $s(d)$ if and only if $y_{j} \in S^{m_{j}}\left(d_{j}\right)$ for all $j$. Next, let $I(M)$ be the set of 
indices $U_{j=1}^{N}\left\{(j, 1), \ldots,\left(j, m_{j}+1\right)\right\}$ and denote the $k$-th component of $x_{j} \in S^{m_{j}}$ by $x_{j, k}$. So, the $\left(k+\sum_{i=1}^{j-1}\left(m_{i}+1\right)\right)-t h$ component of $x \in S$ is denoted by $x_{j, k}$, $k=1, \ldots, m_{j}+1$, i.e.

$$
x=\left(x_{1,1}, x_{1,2}, \ldots, x_{1, m_{1}+1}, x_{2,1}, \ldots, x_{j, k} \cdots, x_{N}, 1, \ldots, x_{N, m_{N}+1}\right)^{\top} .
$$

Further, let the block diagonal (triangulation) matrix $Q$ (of $S$ ) be defined by

$$
Q=\left[\begin{array}{llllll}
Q_{1} & 0 & \cdot & \cdot & \cdot & 0 \\
0 & Q_{2} & \cdot & & & \cdot \\
\cdot & & \cdot & & & \cdot \\
\cdot & & & \cdot & & \cdot \\
\cdot & & & & \cdot & 0 \\
0 & \cdot & . & . & 0 & Q_{N}
\end{array}\right] \text {. }
$$

where $Q_{j}$ is the $\left(m_{j}+1\right) x\left(m_{j}+1\right)$ extended standard triangulation matrix of $s^{m_{j}} \quad$ defined in section 2.6, $j \in I_{N}$. The $\left(k+\sum_{i=1}^{j-1}\left(m_{i}+1\right)\right)-t h$ column of $Q$ will be denoted by $q(j, k), k=1, \ldots, m_{j}+1, j \in I_{N}$. Note that the rank of $Q$ is equal to $M$, which is the dimension of $S$. Using this matrix $Q$ we can define regions $A(T)$ for $T \subset I(M)$ in the same way as described in section 4.5. Recall that in that section $A(T)$ was only defined for $T$ being a proper subset of $I_{n+1}$ and that $A(T)$ was triangulated by the standard triangulation of $\mathrm{s}^{n} \cdot \mathrm{Als}$, for proper $T \subset I_{n+1}$ the rank of the matrix with columns $q(i), i \in T$, was equal to $|T|$. In this chapter we will have the same situation except that a priori a triangulation of $\mathrm{S}$ is not available and that $\mathrm{T}$ must be restricted to special subsets of $I(M)$. The main idea is to take only subsets $T$ such that the matrix with columns $q(j, k),(j, k) \in T$, has full rank $|T|$. Then we show that $S$ is the union of these $A(T)$. Finally, we triangulate each $A(T)$ in the standard way by using only the columns $q(j, k),(j, k) \in T$. We will prove that the union of these triangulations triangulates the set $s$. Since each $A(T)$ is triangulated in a different way the triangulation of $S$ looks very complicated. However, after explaining the algorithm it will become clear that, given the starting point, the triangulation of $S$ is very natural.

First we take an arbitrary grid point $y \in S(d)$. This point will be the starting point of the algorithm. Next we define the regions $A(T)$ for 
"proper" т.

DEFINITION 6.2.1. Let $\mathrm{z}$ be the collection of subsets $\mathrm{T}$ of $\mathrm{I}(\mathrm{M})$ such that for all $j \in I_{N}$ there is at least one element $(j, k)$ not in $T$. Then for $T \in Z$ the region $A(T)$ of $S$ is defined by

$$
A(T)=\left\{x \in S \mid x=y+\underset{\substack{\left.(j, k) \in T \\ \text { nonnegative numbers } \lambda_{j, k}\right\}}}{ }\right. \text {. }
$$

Clearly, $T \in Z$ if and only if the rank of the matrix with columns $q(j, k)$, $(j, k) \in T$, is equal to $|T|$. We call a point $x$ proper in the region $A(T)$ if $\lambda_{j, k}>0$ for all $(j, k) \in T$. The set of proper points of $A(T)$ will be denoted by $\AA(T)$. Note that int $A(T)$ is not equal to $\AA(T)$. Clearly, each point $x$ is a proper point of exactly one region $A(T)$, i.e. the subsets $\AA(T), T \in Z$, partition $S$. Moreover, since the matrix $[q(j, k),(j, k) \in T]$ has full rank, the $\lambda_{j, k}$ 's are unique. For $N=2, m_{1}=m_{2}=1, d_{1}=4$ and $d_{2}=6$, the regions $A(T), T \in Z$, are illustrated in figure 6.2 .3 , such that $\mathrm{y}=(3 / 4,1 / 4,2 / 3,1 / 3)^{\top}$. Recall that $\AA(\varnothing)=A(\varnothing)=\{y\}$.

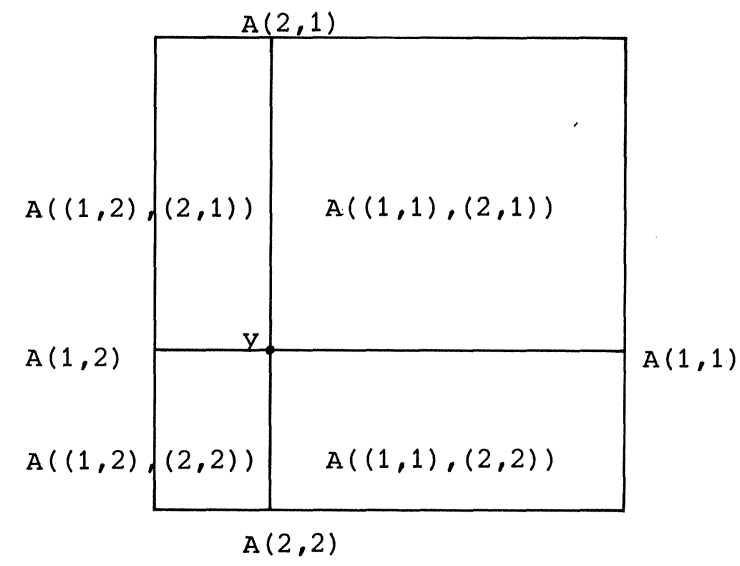

Figure 6.2.3. The regions $A(T), T \in Z$, if $N=2, m_{1}=m_{2}=1, d_{1}=4, d_{2}=6$, $y=(3 / 4,1 / 4,2 / 3,1 / 3)^{\top}$. 
COROLLARY 6.2.2. The sets $\AA(T)$ partition $S, T \in Z$. Moreover for all $T_{1}$ and $\mathrm{T}_{2} \in \mathrm{Z}$

$$
A\left(T_{1}\right) \cap A\left(T_{2}\right)=A\left(T_{1} \cap T_{2}\right)
$$

Now we first triangulate those $A(T)$ with $\AA(T) \neq \varnothing$. Note that if $\AA(T)=\varnothing$ there is a unique subset $T_{1}$ of $T$ such that $A\left(T_{1}\right)=A(T)$ and $\AA\left(T_{1}\right) \neq \varnothing$.

DEFINITION 6.2.3. For $T \in \mathrm{Z}$ with $|\mathrm{T}|=\mathrm{t}$ such that $\stackrel{\circ}{\AA}(\mathrm{T}) \neq \emptyset, \mathrm{G}(\mathrm{T})$ is the collection of $t$-simplices $\sigma\left(y^{1}, \pi(T)\right)$ with vertices $y^{1}, \ldots, y^{t+1}$ in $s$ such that

$$
\begin{aligned}
& y^{1}=y+\sum_{(j, k) \in T} d_{j}^{-1} \mu_{j, k} q(j, k) \text { for nonnegative integers } \mu_{j, k^{\prime}} \\
& \pi(T)=\left(\pi_{1}, \ldots, \pi_{t}\right) \text { is a permutation of the elements of } T, \\
& y^{i+1}=y^{i}+D q\left(\pi_{i}\right) \quad i=1, \ldots, t,
\end{aligned}
$$

where $D$ is the $(M+N)$-diagonal matrix with $(j, k)$-th diagonal element equal to $d_{j}^{-1}, j \in I_{N}$. If $\stackrel{\circ}{\AA}(T)=\varnothing$ define $G(T)$ as the collection of $t_{1}$-simplices of $G\left(T_{1}\right)_{r}$ where $T_{1}$ with $\left|T_{1}\right|=t_{1}$ is the unique subset of $T$ such that $A(T)=A\left(T_{1}\right)$ and $\stackrel{\AA}{A}\left(T_{1}\right) \neq \varnothing$.

Observe that $\operatorname{Dq}\left(\pi_{i}\right)=d_{j}^{-1} \cdot q(j, k)$ if $\pi_{i}=(j, k)$. Hence, all vertices of $\sigma\left(y^{1}, \pi(T)\right) \quad$ defined above are elements of $S(d)$.

THEOREM 6.2.4. For any $T \in \mathrm{z}$ with $\stackrel{\AA}{\mathrm{A}}(\mathrm{T}) \neq \emptyset$, the collection $\mathrm{G}(\mathrm{T})$ of t-simplices $\sigma\left(\mathrm{Y}^{1}, \pi(\mathrm{T})\right)$ triangulates $\mathrm{A}(\mathrm{T})$.

PROOF. Since $\mu_{j, k} \geq 0$ for all $(j, k) \in T, y^{1} \in A(T)$. Because of $(i i)$ and (iii) we have also that $y^{i} \in A(T), i \geq 2$. Hence, $\sigma\left(y^{1}, \pi(T)\right)$ is a simplex of $A(T)$.

Conversely, let $x$ be an arbitrary point in $A(T)$. So, there are unique nonnegative numbers $\lambda_{j, k^{\prime}}(j, k) \in T$, such that

$$
x=y+\sum_{(j, k) \in T} \lambda j, k^{q(j, k)}
$$

Let $y^{1}=y+\sum d_{j}^{-1} \mu_{j, k} q(j, k)$ such that $\mu_{j, k}$ is the greatest integer less than $d_{j} \lambda_{j, k}$. If $\lambda_{j, k}$ is equal to zero, we take $\mu_{j, k}=0$. Clearly $y^{1} \in A(T)$. 
Define now

$$
z(j, k)=d_{j} \lambda_{j, k}-\mu_{j, k}
$$

and let $\pi(T)$ be a permutation of the elements of $T$ such that both

$$
z\left(\pi_{1}\right) \geq z\left(\pi_{2}\right) \geq \ldots \geq z\left(\pi_{t}\right)
$$

and the vertices $y^{i+1}=y^{i}+D q\left(\pi_{i}\right), i=1, \ldots, t$, are points in $A(T)$.

Since $x \in S$ there exists at least one such permutation if $\AA(T)$ is nonempty. Furthermore, let

$$
\begin{aligned}
& \beta_{1}=1-z\left(\pi_{1}\right) \\
& \beta_{i}=z\left(\pi_{i-1}\right)-z\left(\pi_{i}\right) \quad i=2, \ldots, t \\
& \text { and } \\
& \beta_{t+1}=z\left(\pi_{t}\right) . \\
& \text { Clearly, } \sum_{i=1}^{t+1} \beta_{i}=1, \beta_{i} \geq 0 \text { for all } i \text {, and } \\
& \sum_{i=1}^{t+1} \beta_{i} y^{i}=y^{1}+\sum_{i=2}^{t+1} \beta_{i}\left(y^{i}-y^{1}\right)=y^{1}+\sum_{i=2}^{t+1} \beta_{i}\left\{\sum_{j=2}^{i}\left(y^{j}-y^{j-1}\right)\right\} \\
& =y^{1}+\sum_{j=2}^{t+1}\left(y^{j}-y^{j-1}\right)\left(\sum_{i=j}^{t+1} \beta_{i}\right) \\
& =y^{1}+\sum_{j=2}^{t+1} \operatorname{Dq}\left(\pi_{j-1}\right) z\left(\pi_{j-1}\right) \\
& =y+\sum_{(j, k) \in T} \mu_{j, k} d_{j}^{-1} q(j, k)+ \\
& \left.\sum_{(j, k) \in T} d_{j}^{-1} q(j, k)\left\{d_{j} \lambda_{j, k}-\mu_{j, k}\right)\right\} \\
& =y+\sum_{(j, k) \in T} d_{j} \lambda_{j, k} d_{j}^{-1} q(j, k)=x .
\end{aligned}
$$


Hence, $\mathrm{x} \in \sigma\left(\mathrm{y}^{1}, \pi(\mathrm{T})\right)$ while $\sigma \in \mathrm{G}(\mathrm{T})$. Moreover $\mathrm{x}$ lies in the (unique) face of $\sigma$ whose vertices are the $y^{i}$ is with positive $\beta_{i}$. Therefore the open faces of the simplices of $G(T)$ cover $A(T)$. It remains to prove that there is exactly one open face containing $x$. For each number $\gamma \in(0,1]$ let $w(\gamma)$ be the t-vector defined by

$$
\begin{aligned}
w_{j, k}(\gamma) & =\left\lfloor d_{j} \lambda_{j, k}\right\rfloor+1 & \text { if } & d_{j} \lambda_{j, k}-\left\lfloor d_{j} \lambda_{j, k}\right\rfloor \geq \gamma \\
& =\left\lfloor d_{j} \lambda_{j, k}\right\rfloor & & \text { otherwise. }
\end{aligned}
$$

Clearly, each vertex $y^{i}$ of a simplex in $G(T)$ containing $x$ and having a positive weigth $\beta_{i}$ is of the form (cf. Todd [1976a, page 31])

$$
y^{i}=y+\sum_{(j, k) \in m} w_{j, k}(\gamma) d_{j}^{-1} q(j, k) \quad \text { for some } \gamma \in(0,1] \text {. }
$$

Moreover, the number of different $w(\gamma)$ 's for $\gamma \in(0,1]$ is equal to the number of positive $\beta_{i}$ 's. Since the vector $w(\gamma)$ is determined without reference to $\mathrm{y}^{1}$ and $\pi(T)$, the point $\mathrm{x}$ must lie in exactly one open face. This proves that the open faces partition $A(T)$.

Let $\mathrm{z}^{1} \subset \mathrm{z}$ be such that $\operatorname{dim} \mathrm{A}(\mathrm{T})=\mathrm{M}$ iff $\mathrm{T} \in \mathrm{z}^{1}$. We now prove that $\mathrm{S}$ is triangulateu by the union of the triangulations of $A(T), T \in Z^{1}$. Recall from corollary 6.2.2 that $S$ is the union of the regions $A(T), T \in Z$. Hence, since $A\left(T_{1}\right) \subset A\left(T_{2}\right)$ if $T_{1} \subset T_{2}$, the union of the simplices of the triangulations of $A(T), T \in z^{1}$, is equal to $S$. So, it remains to prove that the intersection of two M-simplices $\sigma_{1}$ and $\sigma_{2}$ is a common face or empty. If both $\sigma_{1}$ and $\sigma_{2}$ are simplices of $A(T)$ for some $T \in z^{1}$, then the result follows from theorem 6.2.4. To prove that $\sigma_{1} \cap \sigma_{2}$ is a common face (or is empty) if $\sigma_{1} \in G\left(T_{1}\right)$ and $\sigma_{2} \in G\left(T_{2}\right)$, it is sufficient to show that the triangulations of $A\left(T_{1}\right)$ and $A\left(T_{2}\right)$ are consistent on there intersection $A\left(T_{1}\right) \cap A\left(T_{2}\right)=A\left(T_{1} \cap T_{2}\right)$. In particular, we will prove that $G\left(T_{1}\right)$ and $G\left(T_{2}\right)$ induce the same triangulation $G\left(T_{1} \cap T_{2}\right)$ of $A\left(T_{1} \cap T_{2}\right)$. Of course, if $\mathrm{T}_{1} \cap \mathrm{T}_{2}=\varnothing, \mathrm{A}\left(\mathrm{T}_{1} \cap \mathrm{T}_{2}\right)=\{\mathrm{Y}\}$. 
THEOREM 6.2.5. Let $\mathrm{T}_{1}$ be a proper subset of $\mathrm{T}_{2} \in \mathrm{z}^{1}$. Then $\mathrm{G}\left(\mathrm{T}_{1}\right)$ is the triangulation of $A\left(T_{1}\right)$ induced by $G\left(T_{2}\right)$.

PROOF. Let $\sigma\left(\mathrm{Y}^{1}, \pi\left(\mathrm{T}_{2}\right)\right)$ be a simplex of $\mathrm{G}\left(\mathrm{T}_{2}\right)$ such that $\sigma \cap A\left(\mathrm{~T}_{1}\right) \neq \emptyset$. We prove that $\sigma \cap A\left(T_{1}\right)$ is a face of both $G\left(T_{1}\right)$ and $G\left(T_{2}\right)$. Let $y^{1}, \ldots, y^{M+1}$ be the vertices of $\sigma$. For unique nonnegative integers $\mu_{j, k^{\prime}}(j, k) \in T_{2}$, we have

$$
y^{1}=y+\sum_{(j, k) \in T_{2}} d_{j}^{-1} \mu_{j, k} q(j, k) .
$$

Moreover,

$$
y^{i+1}=y^{1}+\sum_{j=1}^{i} D q\left(\pi_{j}\right) \quad i=1, \ldots, M .
$$

Therefore, $\mathrm{y}^{i} \notin A\left(\mathrm{~T}_{1}\right)$ implies $\mathrm{y}^{j} \notin A\left(\mathrm{~T}_{1}\right)$ for $j \geq i$, and $\mathrm{y}^{i} \in A\left(\mathrm{~T}_{1}\right)$ implies $y^{j} \in A\left(T_{1}\right)$ for $j \leq i$. So, $y^{1} \in A\left(T_{1}\right)$ and there exist an integer $h$ with $1<\mathrm{h}<\mathrm{M}+1$ such that

$$
y^{1}, \ldots, y^{h} \in A\left(T_{1}\right) \text { and } y^{h+1}, \ldots, y^{M+1} \notin A\left(T_{1}\right)
$$

In other words, $\sigma \cap A\left(T_{1}\right)$ is the face $\tau\left(y^{1}, \ldots, y^{h}\right)$ of $\sigma$ in $G\left(T_{2}\right)$. Observe that $h \leq t_{1}<t_{2}=M$ where $t_{i}=\left|T_{i}\right|, i=1,2$. Finally we prove that $\tau\left(y^{1}, \ldots, y^{h}\right)$ is also a face of the triangulation $G\left(T_{1}\right)$ of $A\left(T_{1}\right)$. Since $\mathrm{y}^{1}, \ldots, \mathrm{y}^{\mathrm{h}} \in \mathrm{A}\left(\mathrm{T}_{1}\right)$, we have that

$$
\mu_{j, k}=0 \text { for }(j, k) \notin T_{1}, \text { and } \pi_{i} \in T_{1} \text { for } i=1, \ldots, h \text {. }
$$

Consequently,

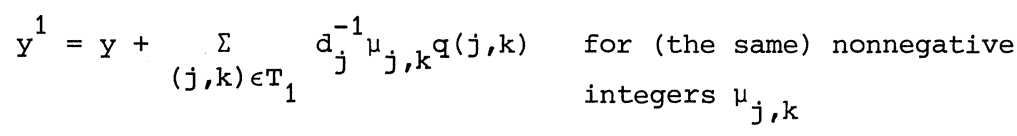

and

$$
y^{i+1}=y^{i}+D q\left(\pi_{i}\right) \quad i=1, \ldots, h-1
$$

for the permutation $\pi^{*}=\left(\pi_{1}, \ldots, \pi_{h-1}\right)$ which must be a permutation of $h-1$ elements of $T_{1}$. Therefore $\tau\left(y^{1}, \ldots, y\right)$ is a face of any simplex $\sigma\left(y^{1}, \pi^{*}\left(T_{1}\right)\right)$ of $G\left(T_{1}\right)$ such that $\pi^{*}\left(T_{1}\right)=\left(\pi^{*}, \pi_{h}^{*}, \ldots, \pi_{t_{1}}^{*}\right)$ is a permutation of the $t_{1}$ elements of $T_{1}$. Hence $\tau$ is also a face of the triangulation $G\left(T_{1}\right)$ of $A\left(T_{1}\right)$. 
This completes the proof.

COROLLARY 6.2.6. The product space $S$ is triangulated by the union of triangulations $G(T)$ of $A(T), T \in z^{1}$.

The triangulation of $\mathrm{S}$ is illustrated in figure 6.2 .4 for $\mathrm{N}=2$, $\mathrm{m}_{1}=\mathrm{m}_{2}=1, \mathrm{~d}_{1}=4, \mathrm{~d}_{2}=6$ and $\mathrm{y}=(3 / 4,1 / 4,2 / 3,1 / 3)^{\top}$, in figure $6.2 .5 \mathrm{a}$ for $\mathrm{N}=2, \mathrm{~m}_{1}=2, \mathrm{~m}_{2}=1, \mathrm{~d}_{1}=1, \mathrm{~d}_{2}=2$ and $\mathrm{y}=\left(1,0,0, \frac{1}{2}, \frac{1}{2}\right)^{\top}$, and in figure $6.2 .5 \mathrm{~b}$ for $\mathrm{N}=2, \mathrm{~m}_{1}=2, \mathrm{~m}_{2}=1, \mathrm{~d}_{1}=2, \mathrm{~d}_{2}=1$ and $y=\left(\frac{1}{2}, \frac{1}{2}, 0,1,0\right)^{\top}$.

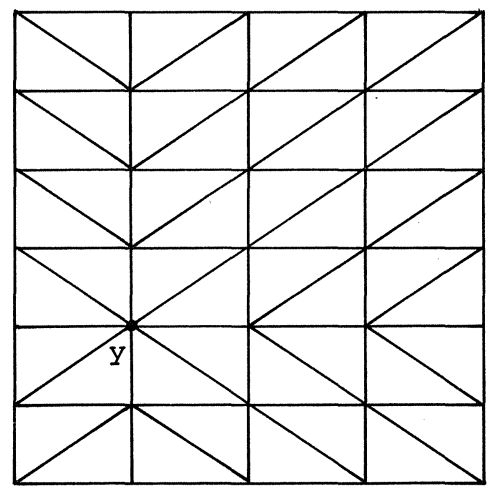

Figure 6.2.4. Triangulation of $\mathrm{s}$ if $\mathrm{N}=2, \mathrm{~m}_{1}=\mathrm{m}_{2}=1, \mathrm{~d}_{1}=4, \mathrm{~d}_{2}=6$, $y=(3 / 4,1 / 4,2 / 3,1 / 3)^{\top}$.

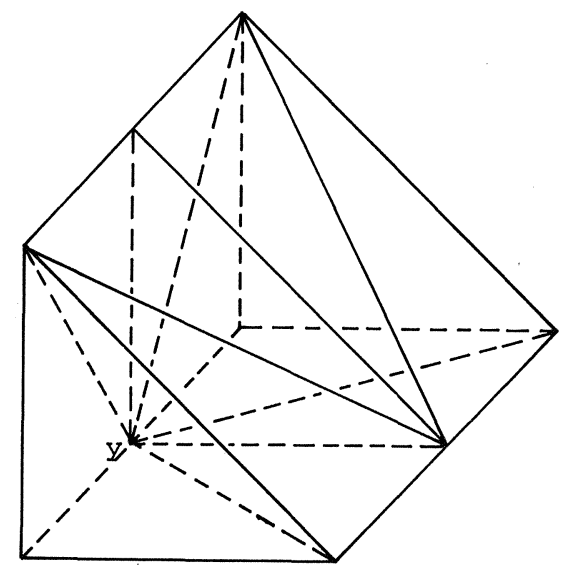

Figure 6.2.5a. Triangulation of $\mathrm{s}$ if $\mathrm{N}=2, \mathrm{~m}_{1}=2, \mathrm{~m}_{2}=1, \mathrm{~d}_{1}=1, \mathrm{~d}_{2}=2$, $y=\left(1,0,0, \frac{1}{2}, \frac{1}{2}\right)^{\top}$. 


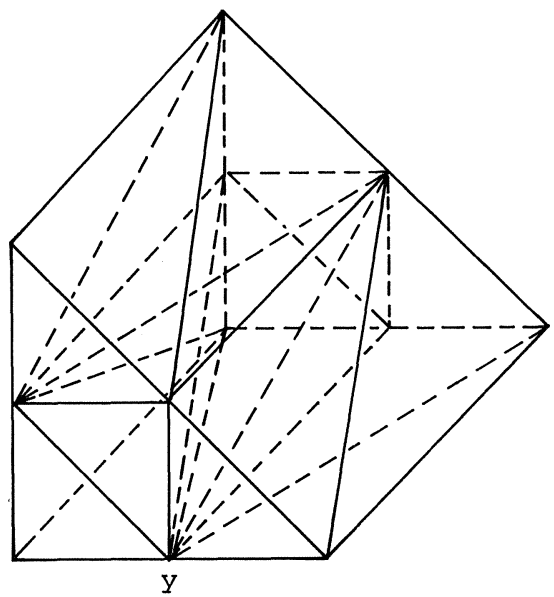

Figure 6.2.5b. Triangulation of $\mathrm{s}$ if $\mathrm{N}=2, \mathrm{~m}_{1}=2, \mathrm{~m}_{2}=1, \mathrm{~d}_{1}=2, \mathrm{~d}_{2}=1$, $\mathrm{y}=\left(\frac{1}{2}, \frac{1}{2}, 0,1,0\right)^{\top}$.

Observe that if $m_{i}=1$ for all $i \in I_{n}$, $S$ corresponds to the unit cube of $R^{N}$. The triangulation is then similar to the $K^{\prime}$ triangulation of $R^{N}$ proposed by Todd [1978a] (see also Van der Laan and Talman [1978b]). An efficient restart algorithm in $\mathrm{R}^{\mathrm{n}}$ based on the $\mathrm{K}^{\prime}$ triangulation is developed in chapter 7. Notice the dependence of the triangulation of $S$ on the (starting) point $y$. In the next section we assign to each point a label being an element of the set I(M). Then the path of adjacent simplices generated by the algorithm (to be described in section 4) is such that the common facets are T-complete (in the sense of section 4.5) when simplices are generated of the triangulation $G(T)$ of $A(T), T \in Z$. As soon as a label $(j, k)$ is found such that the new label set $T \cup\{(j, k)\} \notin \mathrm{z}$, the algorithm terminates with an approximate fixed point.

\subsection{INTEGER LABELLING AND APPROXIMATION.}

Assume we want to compute a fixed point of a continuous function $f$ from $S$ into itself. Therefore $S$ is triangulated as described in the previous section and each (grid) point is labelled as follows. 
DEFINITION 6.3.1. (STANDARD INTEGER LABELLING ON S). A point $\mathrm{x} \in \mathrm{S}$

receives the integer label $l(x)$ where

$$
\ell(x)=\operatorname{lexicomin}\left\{(j, k) \mid f_{j, k}(x)-x_{j, k}=\min _{(i, h) \in I(M)}\left(f_{i, h}(x)-x_{i, h}\right)\right\}
$$

where lexicomin means the lexicographic smallest index.

Observe that $x_{j, k}=0$ implies $l(x) \neq(j, k)$ except in the case that $x$ is a fixed point and $x_{1,1}=0$. Then we assign to $x$ the least index $(1, k)$ such that $x_{1, k}>0$. Recall that $(j, k)$ means $k+\sum_{i=1}^{j-1}\left(m_{i}+1\right)$. Although $s$ is $M$-dimensional, we now have $M+N$ different labels. Note that it can occur that no (grid) point has label $(j, k)$ for certain $(j, k) \in I(M)$.

DEFINITION 6.3.2. A ( $t-1$ ) simplex $\sigma$ is $T$-complete, $T \subset I(M)$, if the vertices of $\sigma$ carry all the labels which are elements of $T(|T|=t)$.

As mentioned in the introduction it makes no sense to define a completely labelled simplex since the number of labels is greater than the number of vertices of a full-dimensional simplex (except if $\mathrm{N}=1$ ). However, to recognize simplices yielding an approximate fixed point we give the following definition.

DEFINITION 6.3.3. A simplex $\sigma$ is a j-stopping simplex for some $j \in I_{N^{\prime}}$ when $(j, k)$ is an element of the label set of $\sigma$ for all $k$.

Observe that a simplex $\sigma$ can be a j-stopping simplex for several j's. If $\sigma$ is a j-stopping simplex, then $m_{j} \leq \operatorname{dim} \sigma \leq \mathrm{M}$.

COROLLARY 6.3.4. A T-complete simplex $\sigma$ is a stopping simplex if and only if $\mathrm{T} \notin \mathrm{z}$.

In the next theorem we prove that a $j$-stopping simplex is a good approximation of a fixed point of $f$. Let $G$ be the triangulation defined in the previous section. 
THEOREM 6.3.5. Let $\varepsilon, \delta>0$ be such that $\max _{j, k}\left|x_{j, k}{ }^{-y_{j, k}}\right| \leq \delta$ and

$$
\max _{(j, k) \in I(M)}\left|f_{j, k}(x)-f_{j, k}(y)\right|<\varepsilon \quad \text { for all } x, y \in \sigma, \sigma \in G .
$$

Then for any $x$ in a j-stopping simplex $\sigma$ in $G$

$$
\max _{(j, k) \in I(M)}\left|f_{j, k}(x)-x_{j, k}\right|<(\varepsilon+\delta)\left(m_{j}+1\right) \max _{i \neq j} m_{i} .
$$

PROOF. Let $w^{k}$ be the vertex of $\sigma$ with $\ell\left(w^{k}\right)=(j, k), k=1, \ldots, m_{j}+1$. Since for any $y \in S$

$$
\sum_{k=1}^{m_{j}+1}\left(f_{j, k}(y)-y_{j, k}\right)=0,
$$

we have

$$
f_{j, k}\left(w^{k}\right)-w_{j, k}^{k} \leq 0 \quad k=1, \ldots, m_{j}+1 .
$$

Let $\mathrm{x}$ be an arbitrary point in $\sigma$.

$$
\begin{aligned}
\text { Since }\left|x_{j, k}-w_{j, k}^{k}\right| \leq \delta \text { and } & \left|f_{j, k}(x)-f_{j, k}\left(w^{k}\right)\right|<\varepsilon \text {, we get } \\
f_{j, k}(x)-x_{j, k}= & \left(f_{j, k}(x)-f_{j, k}\left(w^{k}\right)\right)+\left(f_{j, k}\left(w^{k}\right)-w_{j, k}^{k}\right)+ \\
& +\left(w_{j, k}^{k}-x_{j, k}\right)<\varepsilon+\delta \quad k=1, \ldots, m_{j}+1 .
\end{aligned}
$$

Moreover,

$$
\begin{array}{r}
\text { (6.3.1) } \quad f_{j, k}(x)-x_{j, k}=-\sum_{\substack{h=1 \\
h \neq k}}^{m_{j}+1}\left(f_{j, h}(x)-x_{j, h}\right)>-m_{j}(\varepsilon+\delta) \\
k=1, \ldots, m_{j}+1 .
\end{array}
$$

All this together implies

$$
\max _{k \in I_{m_{j}+1}}\left|f_{j, k}(x)-x_{j, k}\right|<(\varepsilon+\delta) m_{j}<(\varepsilon+\delta)\left(m_{j}+1\right) \max _{i \neq j} m_{i} .
$$

In particular, inequality (6.3.1) holds for $\mathrm{x}=\mathrm{w}^{1}$. Hence, we have for all indices $(i, h), i \neq j$, 


$$
f_{i, h}\left(w^{1}\right)-w_{i, h}^{1} \geq f_{j, 1}\left(w^{1}\right)-w_{j, 1}^{1}>-m_{j}(\varepsilon+\delta) .
$$

Since both $\mathrm{x}$ and $\mathrm{w}^{1}$ lie in $\sigma$, we get

$$
f_{i, h}(x)-x_{i, h}>-\varepsilon-\delta-m_{j}(\varepsilon+\delta)=-\left(m_{j}+1\right)(\varepsilon+\delta) .
$$

On the other side we obtain

$$
f_{i, h}(x)-x_{i, h}=-\sum_{\substack{k=1 \\ k \neq h}}^{m_{i}+1}\left(f_{i, k}(x)-x_{i, k}\right)<m_{i}\left(m_{j}+1\right)(\varepsilon+\delta) .
$$

Combining the last two inequalities we get

$$
\max _{h \in I_{m_{i}+1}}\left|f_{i, h}(x)-x_{i, h}\right|<(\varepsilon+\delta)\left(m_{j}+1\right) m_{i},
$$

which proves the theorem.

The theorem says that a $\mathrm{T}$-complete simplex $\sigma$ for $\mathrm{T} \notin \mathrm{Z}$ yields an approximate fixed point. In the following section an algorithm is presented that will always generate such a simplex and can start anywhere in $\mathrm{S}$.

\subsection{THE VARIABLE DIMENSION ALGORITHM ON S.}

The algorithm to be described in this section is a modification of Van der Laan and Talman's basic algorithm on $S^{n}$. Let $s$ be triangulated as described in section 6.2 for given grid vector $d$ and grid point $y \in S(d)$. Then the algorithm proceeds as follows where $n_{j}$ is the number of labels $(j, k), k \in I_{m_{j}+1}$, already found. Each point $x \in S$ is labelled according to definition 6.3 .1 . 
STEP 0. Set $t=0, T=\varnothing, \pi(T)=\varnothing, y^{1}=y, \sigma=\sigma\left(y^{1}, \pi(T)\right), \bar{y}=y^{1}, n_{j}=0$ for all $j \in I_{N^{\prime}}$ and $R_{j, k}=0$ for all $(j, k) \in I(M)$.

STEP 1. Calculate $\ell(\bar{y})$. If $\ell(\bar{y}) \notin T, a(T \cup\{\ell(\bar{y})\})$-complete simplex is found and go to step 3 . Otherwise $\ell(\bar{y})=\ell\left(y^{s}\right)$ for exactly one vertex $\mathrm{y}^{\mathrm{s}} \neq \overline{\mathrm{y}}$ of $\sigma$. The facet opposite $\mathrm{y}^{\mathrm{s}}$ is $\mathrm{T}$-complete.

STEP 2. If $s=t+1$ and $R_{\pi_{t}}=0$, go to step 4 . Otherwise $\sigma\left(y^{1}, \pi(T)\right)$ and $R$ are adapted according to table 6.4 .1 by replacing $\mathrm{y}^{\mathrm{s}}$. Return to step 1 with $\bar{y}$ equal to the new vertex of $\sigma$.

$\operatorname{STEP} 3$. Let $\ell(\bar{y})=(j, k)$. If $n_{j}=m_{j}, a j$-stopping simplex is found and the algorithm terminates. Otherwise, set $T=T \cup\{(j, k)\}$, $\pi(T)=(\pi(T),(j, k)), \sigma=\sigma\left(y^{1}, \pi(T)\right), n_{j}=n_{j}+1$ and $t=t+1$. Return to step 1 with $\bar{y}$ equal to the new vertex $y^{t+1}=y^{t}+d_{j}^{-1} q(j, k)$ of $\sigma$.

STEP 4. Let $\pi_{t}=(i, h)$. Label $(i, h)$ is deleted. Set $T=T \backslash\{(i, h)\}$, $\pi(T)=\left(\pi_{1}, \ldots, \pi_{t-1}\right), \sigma=\sigma\left(y^{1}, \pi(T)\right), n_{i}=n_{i}-1$ and $t=t-1$. Return to step 2 with $y^{S}$ equal to the vertex of $\sigma$ having label $(i, h)$.

\begin{tabular}{l|l|l|l} 
& $y^{1}$ becomes & $\pi(T)$ becomes & $R$ becomes \\
\hline$s=1$ & $y^{1}+D q\left(\pi_{1}\right)$ & $\left(\pi_{2}, \ldots, \pi_{t}, \pi_{1}\right)$ & $R+e\left(\pi_{1}\right)$ \\
$2 \leq s \leq t$ & $y^{1}$ & $\left(\pi_{1}, \ldots, \pi_{s-2}, \pi_{s}, \pi_{s-1}, \pi_{s+1}, \ldots, \pi_{t}\right)$ & $R$ \\
$s=t+1$ & $y^{1}-D q\left(\pi_{t}\right)$ & $\left(\pi_{t}, \pi_{1}, \ldots, \pi_{t-1}\right)$ & $R-e\left(\pi_{t}\right)$
\end{tabular}

Pable 6.4.1. $\mathrm{s}$ is the index of the vertex to be replaced.

Clearly, each simplex generated by the algorithm is of the form $\sigma\left(y^{1}, \pi(T)\right)$ for some $T \in Z$ such that

$$
y^{1}=y+\sum_{(j, k) \in T} d_{j}^{-1} R_{j, k} q(j, k) \text { with nonnegative integers } R_{j, k^{\prime}}
$$

$$
\begin{aligned}
& \pi(T)=\left(\pi_{1}, \ldots, \pi_{t}\right) \text { is a permutation of the elements of } T, \\
& y^{i+1}=y^{i}+D q\left(\pi_{i}\right) \quad i=1, \ldots, t .
\end{aligned}
$$

Therefore, if $\sigma$ is a simplex in $S$, it satisfies the condition (i)-(iii) of 
section 6.2 , i.e. $\sigma$ is a simplex of the triangulation $G(T)$ of $A(T)$. Moreover, either $\sigma$ has two T-complete facets or $\sigma$ is $(T \cup\{(j, k)\})$-complete in which case it has exactly one T-complete facet $\tau$. In the first case $\sigma$ has two vertices with the same label. One of these two vertices is the new vertex while the other one must be removed yielding a simplex adjacent to $\sigma$ as stated in step 2. In the other case either a new label is found so that a test is made whether to terminate the algorithm or to increase the dimension (step 3 ), or $\sigma$ is a $t$-dimensional facet of a $(t+1)$-simplex $\tilde{\sigma}$ of $G(T \cup\{(j, k)\})$, while the only vertex of $\tilde{\sigma}$ in $\AA(T \cup\{(j, k)\})$ has to be removed. Then step 4 has to be performed, i.e. $(j, k)$ is deleted and the dimension is decreased. Note that as soon as label $(j, k)$ (say) is found a search for the other labels is made by moving to the $(j, k)$-th boundary of $S$ where label $(j, k)$ is missing. In other words, the $(j, k)$-th component is forced to decrease if label $(j, k)$ is found since in that direction new labels can be found. If, however, the path of simplices moves too far away from the $(j, k)$-th boundary of $s$, label $(j, k)$ is deleted from the current label set. Consequently, the algorithm generates a path of adjacent simplices of variable dimension of the triangulation $\mathrm{G}$ of $\mathrm{S}$ such that two adjacent $\mathrm{t}$-simplices whose common facet is $T$-complete are both of the form $\sigma\left(y^{1}, \pi(T)\right)$ and are lying in $A(T)$. If two adjacent simplices have not the same dimension so that one of them is a facet of the other, then the T-complete (say) facet is of the form $\tau\left(y^{1}, \pi\left(T^{\prime}\right)\right)$ and lies in $A\left(T^{\prime}\right)$ where $T^{\prime}=T \backslash\{(j, k)\}$ for some $(j, k) \in T$, whereas the simplex is of the form $\sigma\left(\mathrm{y}^{1}, \pi(T)\right)$ and lies in $A(T)$. Recall that in the latter case $R_{j, k_{t+1}}=0$ and $y^{t+1}=y^{t}+d_{j}^{-1} q(j, k)$ whereas $\tau$ is the facet of $\sigma$ opposite to $y^{t+1}$.

We now prove that the algorithm terminates within a finite number of iterations with a j-stopping simplex for some $j \in I_{N}$. Remember that $A(T)$ is only defined for $T \in Z$. However, as soon as $T$ is not longer an element of $\mathrm{z}$, the algorithm terminates since then a j-stopping simplex is found for some $j$ (according to corollary 6.3.4). To show that all replacement steps are feasible, let $\sigma\left(\mathrm{y}^{1}, \ldots, \mathrm{y}^{\mathrm{t}+1}\right)$ be a simplex of the triangulation $\mathrm{G}(\mathrm{T})$ of $A(T)$ such that the facet $\tau\left(y^{1}, \ldots, y^{s-1}, y^{s+1}, \ldots, y^{t+1}\right)$ is on the $(j, k)$-th boundary of $S$ for some $(j, k) \in I(M)$ and $y_{j, k}^{S}>0$. Suppose that $\tau$ is $T$-complete. We now prove that $y^{S}$ can be only removed if $s=t+1$, $\pi_{t}=(j, k-1)$ and $R_{j, k-1}=0\left(k-1=m_{j}+1\right.$ if $\left.k=1\right)$ so that the algorithm performs step 4 (by deleting the vertex $y^{t+1}$ and label $(j, k-1)$ ). Since $\tau$ is $\mathrm{T}$-complete and the labelling rule is proper, $(j, k) \notin T$. However, $s<t+1$ 
implies $y^{s+1}=y^{S}+d_{j}^{-1} q(j, k)$ and so $\pi_{s}=(j, k) \in T$, which gives a contradiction. Hence $s=t+1$ and $y^{s}=y^{s-1}+d_{j}^{-1} q(j, k-1)$ which implies $\pi_{t}=(j, k-1)$. Moreover, since $y_{j, k}^{1}=0$ and $(j, k) \notin T, R_{j, k-1}$ must be equal to zero (and $y_{j, k}$ also). This proves the feasibility of the replacement step in step 2 .

Next we prove that step 3 is always feasible. If $y_{j, k}^{t}=0$ and $l\left(y^{s}\right)=(j, k)$ is a new label, the extension step cannot be performed. However, since $(j, k)$ is the new label, we have $\pi_{i} \neq(j, k), i=1, \ldots, t-1$ (and $R_{j, k}$ is equal to zero). Consequently, since $y^{1}, \ldots, y^{t}$ are vertices of $s$ and $\pi_{i} \neq(j, k)$,

$$
0 \leq y_{j, k}^{1} \leq \ldots \leq y_{j, k}^{t}=0 .
$$

This implies that $y_{j, k}^{i}=0$ for all $i$ and hence label $(j, k)$ cannot occur. Finally we prove that the algorithm can never return in a previously generated simplex. Let $\sigma^{0}, \sigma^{1}, \ldots$ be the path of adjacent simplices generated by the algorithm. Then, as mentioned above, for some $T \in Z$ $\sigma^{i}=\sigma\left(\mathrm{y}^{1}, \pi(\mathrm{T})\right)$ and either $\sigma^{i}$ shares $\mathrm{T}$-complete facets with $\sigma^{i-1}$ and $\sigma^{i+1}$ or $\sigma^{i}$ is a $(T \cup\{(j, k)\})$-complete facet of $\sigma^{i-1}$ (or $\sigma^{i+1}$ ) and shares a $T$-complete facet with $\sigma^{i+1}$ (or $\sigma^{i-1}$ ), $i=1,2, \ldots$. To prove that the algorithm cannot cycle, we now show that $\sigma^{0}$ has exactly one adjacent simplex with one of these two properties and that $\sigma^{i}, i \geq 1$, has exactly two such adjacent simplices. If so, then a simplex $\sigma^{i}$ can never be revisited as is easily seen from the arguments used in section 4.3 to prove noncycling for Kuhn's artificial start algorithm. Clearly $\sigma\left(\mathrm{y}^{1}, \pi(\mathrm{T})\right)$ with $\mathrm{y}^{1}=\mathrm{y}$ and $\mathrm{T}=\{\ell(\mathrm{y})\}$ is the only simplex of $G(T)$ adjacent to $\sigma^{0}=\{y\}$ having one of the two properties. If $i>0$, let $\sigma^{i}$ have two T-complete facets $\tau_{1}$ and $\tau_{2}$. Obviously, simplices adjacent to $\sigma^{i}$ and not sharing $\tau_{1}$ or $\tau_{2}$ with $\sigma^{i}$ cannot have one of the two properties since their intersection with $\sigma^{i}$ is not T-complete. If $\tau_{1}$ (or $\tau_{2}$ ) is not in the boundary of $A(T)$, there is a unique simplex $\sigma$ adjacent to $\sigma^{i}$ sharing the facet $\tau_{1}$ ( or $\tau_{2}$ ) such that $\sigma$ has one of the two properties, viz. the t-simplex $\sigma(\tilde{y}, \tilde{\pi}(T))$ obtained from table 6.4 .1 by replacing the vertex of $\sigma^{i}$ opposite to $\tau_{1}$ (or $\tau_{2}$ ). If $\tau_{1}$ (or $\tau_{2}$ ) is in the boundary of $A(T), \tau_{1}$ (or $\tau_{2}$ ) is a $T$-complete $(t-1)$-simplex of $A(T \backslash\{(j, k)\}$ ) for some $(j, k) \in T$ such that $\tau_{1}$ (or $\tau_{2}$ ) is the facet of $\sigma^{i}$ opposite to $y^{t+1}$. In that case $\tau_{1}$ (or $\left.\tau_{2}\right)$ is of the form $\tau\left(y^{1}, \pi(T \backslash\{(j, k)\})\right.$ ) and is a simplex of $G(T \backslash\{(j, k)\})$ so that this simplex is adjacent to $\sigma^{i}$ and has one of the two properties. Clearly, there is no other simplex adjacent to $\sigma^{i}$ sharing 
$\tau_{1}$ (or $\tau_{2}$ ) and having one of these properties.

If $\sigma^{i}(i>0)$ is a $(T \cup\{(j, k)\})$-complete simplex $\sigma\left(y^{1}, \pi(T)\right)$ of $G(T)$, then $\sigma^{i}$ is a facet of exactly one $(t+1)$-simplex $\sigma\left(y^{1}, \pi\left(T^{\prime}\right)\right)$ of $G\left(T^{\prime}\right)$ where $T^{\prime}=T \cup\{(j, k)\}$ (see theorem 2.2.8). Hence, since $\sigma^{i}$ is a $T^{\prime}$-complete facet of $\sigma$, the latter simplex satisfies one of the two properties above (depending on what the label of the new vertex $y^{t+2}$ of $\sigma$ is). Further, let $\tau$ be the facet of $\sigma^{i}$ which is T-complete. Then there is, following the arguments above, only one adjacent simplex $\rho$ of $\sigma^{i}$ sharing $\tau$ with $\sigma^{i}$ such that $\rho$ has one of the two properties. Obviously there are no other simplices adjacent to $\sigma^{i}$ having one of these properties. Combining these results together, we find that the algorithm generates a unique feasible path of adjacent simplices of $\mathrm{G}$ of variable dimension. since the number of simplices in $S$ is finite, the algorithm must terminate, which can only occur if for some $j$ a $j$-stopping simplex is found. The algorithm is illustrated in figure 6.4 .1 for $\mathrm{N}=2, \mathrm{~m}_{1}=\mathrm{m}_{2}=1$, $\mathrm{d}_{1}=4, \mathrm{~d}_{2}=6$ and $\mathrm{y}=(3 / 4,1 / 4,2 / 3,1 / 3)^{\top}$.

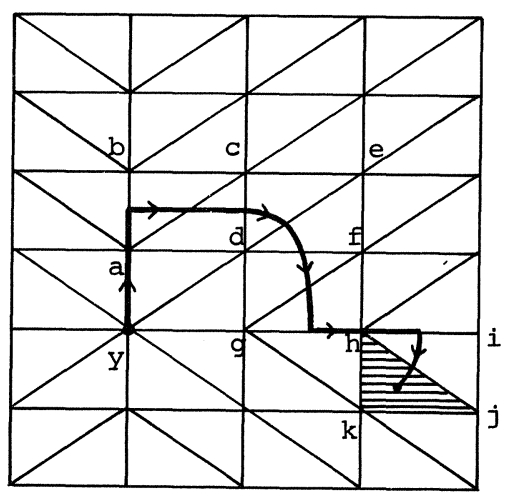

Figure 6.4.1. Illustration of the algorithm on $\mathrm{s} . \mathrm{N}=2, \mathrm{~m}_{1}=\mathrm{m}_{2}=1$, $\mathrm{d}_{1}=4, \mathrm{~d}_{2}=6, \mathrm{y}=(3 / 4,1 / 4,2 / 3,1 / 3)^{\top}$.

In this example $\ell(y)=\ell(a)=\ell(d)=\ell(g)=(2,1)$,

$\ell(b)=\ell(c)=\ell(e)=\ell(f)=\ell(h)=(1,1), \ell(i)=\ell(j)=(2,2)$ and

$\ell(k)=(1,2)$ or $(2,1)$. The simplex with vertices $h, j, k$ is a stopping simplex for the algorithm. Note that if $\ell(a)=(2,2)$ the simplex $\sigma(y, a)$ is a one-dimensional 2-stopping simplex. We emphasize that it is possible that if $\mathrm{S}$ is two-dimensional, four labels are assigned whereas the algorithm terminates with a one-dimensional (at most two-dimensional) 
simplex. Observe that fast movements can be made into all directions while this cannot be done for the "standard" triangulation of $\mathrm{s}$.

Finally, we make some remarks about the path of simplices generated by the algorithm. Let $\sigma\left(y^{1}, \pi(T)\right)$ be a t-simplex of the path. Then for all $(j, k) \in T$ there exists a vertex $w(j, k)$ of $\sigma$ such that

$$
\begin{aligned}
f_{j, k}(w(j, k))-w_{j, k}(j, k) \leq f_{i, h}(w(j, k)) & -w_{i, h}(j, k) \\
& \text { for all }(i, h)
\end{aligned}
$$

since $\sigma$ has at least one $\mathrm{T}$-complete facet. Hence, if $\mathrm{x} \in \sigma$ and $\mathrm{T} \in \mathrm{Z}$, the $(j, k)$-th components of $f(x)-x$ are very close to each other for all $(j, k) \in T$ whereas the other components of $f(x)-x$ are larger (or equal). So, let $C(T)$ be the set of points $z$ in $A(T)$ such that

$$
f_{j, k}(z)-z_{j, k}=\min _{(i, h)}\left\{f_{i, h}(z)-z_{i, h}\right\} \text { if }(j, k) \in T
$$

and

$$
f_{j, k}(z)-z_{j, k}>\min _{(i, h)}\left\{f_{i, h}(z)-z_{i, h}\right\} \quad \text { if }(j, k) \notin T .
$$

Observe that $C(T)$ is not defined for $T \notin Z$. Roughly speaking, if the algorithm generates simplices of the triangulation $\dot{G}(T)$ of $A(T)$, a curve in $C(T)$ is followed until either $f_{j, k}(x)-x_{j, k}$ becomes equal to the minimum for some $(j, k) \notin T$ or the boundary of $A(T)$ is met. In the first case a new label is found and an adjacent curve in $C(T \cup\{(j, k)\})$ is followed with $(t+1)$-simplices. In the other case the point on the boundary of $A(T)$ lies either in bd $S$ or lies in $A(T \backslash\{(i, h)\})$ for some $(i, h) \in T$. If the point lies in bd $S$ it must be a fixed point and the algorithm terminates with a simplex close to that point. Otherwise, as soon as the algorithm generates a $T$-complete facet in $A(T \backslash\{(i, h)\})$, the algorithm continues by following the adjacent curve in $C(T \backslash\{(i, h)\})$ with $(t-1)$-simplices of $G(T \backslash\{(i, h)\})$. If $T$ becomes an element not in $z$, i.e. if a simplex is generated which is a $j$-stopping simplex for some $j$, then it approximates a point $z$ such that 


$$
f_{j, k}(z)-z_{j, k}=\min _{(i, h)}\left\{f_{i, h}(z)-z_{i, h}\right\} \quad \text { for all k. }
$$

Clearly, this can only happen if $z$ is a fixed point of $f$ since $\Sigma_{k}\left(f_{j, k}(z)-z_{j, k}\right)=0$. Note that the algorithm terminates as soon as $T$ becomes an element not of $z$. It can easily be seen that for some given starting point $y$ the path of simplices converges to a chain of adjacent curves in $C(T), T \in Z$, if the mesh goes to zero. This curve starts with the point $y$ and ends with a fixed point of $f$.

6.5. VECTOR LABELLING.

Let $\phi$ be an u.s.c. mapping from $S$ to the set of nonempty, convex sets of $S$ and let $S$ be triangulated as described in section 2 . for some given grid vector $d$ and starting point $y$.

DEFINITION 6.5.1. (STANDARD VECTOR LABELLING ON S): A point $\mathrm{x} \in \mathrm{S}$ receives the $(M+N)$-vector label $\ell(x)$ where

$$
\ell(x)=-f(x)+x+e
$$

where $f(x)$ is an a priori chosen element of $\phi(x)$.

DEFINITION 6.5.2. A $(t-1)$-simplex $\sigma\left(w^{1}, \ldots, w^{t}\right)$ is T-complete with $|\mathrm{T}|=t$ and $T \subset I(M)$, if the system of $M+N$ linear equations

$$
\sum_{i=1}^{t} \lambda_{i} l\left(w^{i}\right)+\sum_{(j, k) \notin T} \mu_{j, k} e(j, k)=e
$$

has a nonnegative solution $\lambda_{i^{\prime}}^{*} i=1, \ldots, t$ and $\mu_{j, k^{\prime}}^{*}(j, k) \notin T$.

Observe that $\mathrm{T}$-completeness is only defined if $t \leq \mathrm{M}+1$. 
LEMMA 6.5 .3 . If $\sigma\left(w^{1}, \ldots, w^{t}\right)$ is a T-complete simplex, then

$$
(M+N) \sum_{i=1}^{t} \lambda_{i}^{*}+\underset{(j, k) \notin T}{\mu_{j, k}^{*}}=M+N \text {. }
$$

PROOF. Summing up all equations we get

$$
\sum_{i=1}^{t} \lambda_{i}^{*}\left\{\sum_{j=1}^{N} \sum_{k=1}^{m_{j}+1}\left(-f_{j, k}\left(w^{i}\right)+w_{j, k}^{i}+1\right)\right\}+\sum_{(j, k) \notin T} \mu_{j, k}^{*}=M+N .
$$

Since $\sum_{k=1}^{m_{j}^{+1}} w_{j, k}^{i}=\sum_{k=1}^{m_{j}+1} f_{j, k}\left(w^{i}\right)=1$ for all $j \in I_{N}$ and for all $i$, we immediately get the desired result.

Again we define a $j$-stopping simplex for $j \in I_{N}$. Note, however, that this definition makes only sense if $\sigma$ is $T$-complete for some $T$.

DEFINITION 6.5.4. A T-complete simplex $\sigma\left(w^{1}, \ldots, w^{t}\right)$ is called a j-stopping simplex if $(j, k) \in T$ for all $k=1, \ldots, m_{j}+1$.

LEMMA 6.5.5. A j-stopping simplex $\sigma$ is a completely labelled simplex, i.e. $\mu_{i, h}^{*}=0$ for all $(i, h) \notin T$.

PROOF. Since $(j, k) \in T$ for all $k$, we obtain by summing up all equations $(j, k)$ over $k$ that

$$
\sum_{i=1}^{t} \lambda_{i}^{*}=1
$$

By summing up all equations $(h, k)$ over $k$ for some $h \neq j$, we get

$$
\left(m_{h}+1\right) \sum_{i=1}^{t} \lambda_{i}^{*}+\sum_{\substack{k=1 \\(h, k) \notin T}}^{m_{h}^{+1}} \mu_{h, k}^{*}=m_{h}+1,
$$

which can only be the case if all $\mu_{h, k}^{*}$ are equal to zero.

COROLLARY 6.5.6. A T-complete simplex is completely labelled if $\mathrm{T} \notin \mathrm{z}$. 
Following the proofs of lemma 4.2 .5 and theorem 4.2.6 we obtain the result that a completely labelled simplex indeed approximates a fixed point. Let $\phi$ be a continuous function $f$.

THEOREM 6.5.7. Let $\varepsilon, \delta>0$ be such that mesh $G=\delta$ and

$$
\max _{(j, k) \in I(M)}\left|f_{j, k}(x)-f_{j, k}(y)\right|<\varepsilon \quad \text { for all } x, y \in \sigma, \sigma \in G .
$$

Then for a completely labelled simplex $\sigma\left(w^{1}, \ldots, w^{t}\right)$ with solution $\lambda^{*}=\left(\lambda_{1}^{*}, \ldots, \lambda_{t}^{*}\right)$ and $x^{*}=\Sigma_{i} \lambda_{i}^{*} w^{i}$ holds

$$
\max _{(j, k) \in I(M)}\left|f_{j, k}\left(x^{*}\right)-x_{j, k}^{*}\right|<\varepsilon .
$$

Moreover, $\mathrm{x}^{\star}$ is a fixed point of the piecewise linear approximation $\bar{f}$ to $f$ with respect to $G$.

If $\phi$ is an u.s.c. mapping, we get an analoguous result (see Todd [1976a]). We now give a short description how to apply the algorithm for vector labelling. Starting in an arbitrarily chosen grid point $y$ the algorithm calculates $\ell(y)$ and makes a pivot step by bringing $\ell(y)$ in the system of linear equations

$$
\sum_{(j, k)} \mu_{j, k} e(j, k)=e
$$

where the sum is over all indices $(j, k)$. If the $(i, h)$-th unit column is eliminated, the simplex $\sigma\left(y^{1}, \pi(T)\right)$ with $y^{1}=y$ and $T=\{(i, h)\}$ is generated and $\ell\left(y^{2}\right)$ is calculated. Then a pivot step is made with $\ell\left(y^{2}\right)$. In general, if a unit column e $(j, k)$ (say) is eliminated by a pivot step, the dimension is increased by adding the vertex $y^{t+2}=y^{t+1}+d_{j}^{-1} q(j, k)$ to the current simplex $\sigma\left(\mathrm{y}^{1}, \pi(\mathrm{T})\right)$ of $G(T)$. Note that $\sigma$ is $(T \cup\{(j, k)\})-$ complete. If, however, a vector $\ell\left(y^{S}\right)$ is eliminated by a pivot step, then the facet opposite to $\mathrm{y}^{\mathrm{s}}$ is $\mathrm{T}$-complete so that $\mathrm{y}^{\mathrm{S}}$ is removed. If $\mathrm{s}=\mathrm{t}+1$ and $R_{\pi_{t}}=0$, the last vertex $y^{t+1}$ of $\sigma$ is deleted and the dimension of $\sigma$ is decreased. Now a pivot step is made by bringing $e\left(\pi_{t}\right)$ back in the current system of linear equations. Note that the facet of $\sigma$ opposite 
$y^{t+1}$ is a $T$-complete simplex of the triangulation $G\left(T \backslash\left\{\pi_{t}\right\}\right)$ of $A\left(T \backslash\left\{\pi_{t}\right\}\right)$. If not both $s=t+1$ and $R_{\pi_{t}}=0$, the standard replacement step is performed by replacing $y^{S}$ according to table 6.4.1. This step yields a new vertex $\bar{y}$ and a pivot step is made with $\ell(\bar{y})$, etc. Hence, as long as all steps are unique and feasible, the algorithm generates a unique. path of adjacent simplices of variable dimension such that for some $T \in \mathrm{Z}$ a simplex $\sigma$ is of the form $\sigma\left(\mathrm{y}^{1}, \pi(T)\right) \in \mathrm{G}(\mathrm{T})$ and either has fust two different $T$-complete facets or is $(T \cup\{(j, k)\})$-complete for some $(j, k) \notin T$. The algorithm terminates as soon as all $\mu_{j, k}^{*}$ are equal to zero, as will be the case when $T \cup\{(j, k)\} \notin z$. Note that if $|T|>M, T$ cannot be an element of $\mathrm{z}$, i.e. if full-dimensional simplices are generated and one of the remaining $N$ unit columns is eliminated, then all $\mu_{j, k}^{*}$ become equal to zero so that a completely labelled simplex is found.

Next we prove that the algorithm indeed terminates within a finite number of steps with a completely labelled simplex. Clearly, since the set of all feasible solutions of the system of linear equations is bounded (see lemma 6.5.3) the pivot steps can always be carried out. Moreover, assuming that by a pivot step only one column is eliminated, the pivot steps are unique. Note that the solution is completely degenerated if a completely labelled simplex is found $(\mathrm{N}>1)$. Hence, the pivot steps are unique and feasible. Now we show that the replacement steps as well as the extension steps are feasible. Clearly, they are all unique.

THEOREM 6.5.8. Let $\sigma\left(y^{1}, \ldots, y^{t+1}\right)$ be a t-simplex of the triangulation $G(T)$ of $A(T)$ such that the facet $\tau\left(y^{1}, \ldots, y^{s-1}, y^{s+1}, \ldots, y^{t+1}\right)$ is on the $(j, k)$-th boundary of $S$ for some $(j, k)$ whereas $y^{S}$ is not on that boundary. If $\tau$ is $T$-complete then :

(i) $\tau$ is completely labelled

or

(ii) $s=t+1, \pi_{t}=(j, k-1)$ and $R_{\pi_{t}}=0$, where $k-1=m_{j}+1$ if $k=1$.

PROOF. Suppose that $\tau$ is $T$-complete but not completely labelled. If $s=t+1$, we have 


$$
y^{t+1}=y^{t}+d_{j}^{-1} q(j, k-1)
$$

since $y^{t}$ lies in the $(j, k)-t h$ boundary of $s$ and $y^{t+1}$ does not. Hence $\pi_{t}=(j, k-1)$ and so $(j, k-1) \in T$. Moreover, $(j, k) \notin T$ and consequently $R_{\pi_{t}}=R_{j, k-1}=0$, which implies (ii). If $s \leq t$, then we must have that

$$
y^{s+1}=y^{s}+d_{j}^{-1} q(j, k)
$$

and so $\pi_{s}=(j, k) \in T$. Consequently, $e(j, k)$ has already been eliminated. Consider now the $(j, k)-t h$ equation of the system of linear equations corresponding to $\tau$,

$$
\sum_{\substack{i=1 \\ i \neq s}}^{t+1} \lambda_{i}\left(-f_{j, k}\left(y^{i}\right)+y_{j, k}^{i}+1\right)=1 .
$$

Since $y_{j, k}^{i}$ is equal to zero for all $i \neq s$, this equation simplifies to

$$
-\sum_{\substack{i=1 \\ i \neq s}}^{t+1} \lambda_{i} f_{j, k}\left(y^{i}\right)+\sum_{\substack{i=1 \\ i \neq s}}^{t+1} \lambda_{i}=1 .
$$

If the total system has a feasible solution $\left(\lambda_{1}^{*}, \ldots, \lambda_{s-1}^{*}, \lambda_{s+1}^{*}, \ldots, \lambda_{t+1}^{*}\right)$, then $\Sigma_{i} \lambda_{i}^{*} \geq 1$ as follows from the latter equation since the first term on the left side is nonpositive. If $\Sigma_{i} \lambda_{i}^{*}=1, \tau$ must be completely labelled (see lemma 6.5.3). If, however, $\Sigma_{i} \lambda_{i}^{*}>1$, then by the same lemma the system has no feasible solution implying that $\tau$ is not T-complete.

The theorem says that if such a $\sigma$ is generated by the algorithm while $\ell\left(y^{s}\right)$ is eliminated the facet $\tau$ is completely labelled (in which case the algorithm terminates) or step 4 has to be performed. Hence, the replacement step is always feasible. Following the same arguments it can be easily proved that an extension step is also always feasible. Therefore we can conlude that all steps are unique and feasible so that 
the algorithm must terminate, which can only occur if a completely labelled simplex is faund. Note again that the algorithm can terminate with lower-dimensional simplices. Assuming nondegeneracy, this will only happen for a boundary facet (case (1) of theorem 6.5.8). Finally, we give an interpretation of the path followed by the algorithm. Let $\overline{\bar{f}}$ be the piecewise-linear approximation to $\phi$ with respect to the triangulation $G$, such that $\bar{f}(x)=f(x)$ if $x$ is a grid point of $S$.

THEOREM 6.5.9. For $\mathrm{T} \in \mathrm{z}$ let $\tau\left(\mathrm{w}^{1}, \ldots, \mathrm{w}^{\mathrm{t}}\right)$ be a $\mathrm{T}$-complete simplex generated by the algorithm, and let $\lambda^{*}=\left(\lambda_{1}^{*}, \ldots, \lambda_{t}^{*}\right)$ be the solution of the system of equations with respect to $\tau$. Then

$$
\bar{f}_{j, k}\left(w^{*}\right)-w_{j, k}^{*}=\alpha\left(\lambda^{*}\right) \quad(j, k) \in T
$$

and (assuming nondegeneracy)

$$
\bar{f}_{j, k}\left(w^{*}\right)-w_{j, k}^{*}>\alpha\left(\lambda^{*}\right) \quad(j, k) \notin T,
$$

where $w^{*}=\Sigma_{i} \lambda_{i}^{*} w^{i} / \Sigma_{i} \lambda_{i}^{*}$ and $\alpha\left(\lambda^{*}\right)=-\left(1-\Sigma_{i} \lambda_{i}^{*}\right) / \Sigma_{i} \lambda_{i}^{*}$ (if $t=0$ then $\left.\alpha\left(\lambda^{*}\right)=-\infty\right)$.

PROOF. Consider first the $(j, k)-$ th equations of the system, $(j, k) \in T$. Since $e(j, k)$ has been eliminated, we obtain

$$
\sum_{i=1}^{t}-\lambda_{i}^{*}\left(\bar{f}_{j, k}\left(w^{i}\right)-w_{j, k}^{i}\right)+\sum_{i=1}^{t} \lambda_{i}^{*}=1 \quad(j, k) \in T .
$$

Since $\bar{f}$ is linear in $\sigma$ and letting $w^{*}=\sum_{i=1}^{t} \lambda_{i}^{*} w^{i} / \sum_{i=1}^{t} \lambda_{i}^{*}$, we get

$$
\bar{f}_{j, k}\left(w^{*}\right)-w_{j, k}^{*}=-\left(1-\sum_{i=1}^{t} \lambda_{i}^{*}\right) / \sum_{i=1}^{t} \lambda_{i}^{*}=\alpha\left(\lambda^{*}\right) \quad(j, k) \in T .
$$

For $(j, k) \notin T$ we have in the same way

$$
\sum_{i=1}^{t} \lambda_{i}^{*}\left(-\bar{f}_{j, k}\left(w^{i}\right)+w_{j, k}^{i}\right)+\sum_{i=1}^{t} \lambda_{i}^{*}+\mu_{j, k}^{*}=1 \text { with } \mu_{j, k}^{*}>0 .
$$


Hence,

$$
\overline{\mathrm{f}}_{j, k}\left(w^{*}\right)-w_{j, k}^{*}=\alpha\left(\lambda^{*}\right)+\mu_{j, k}^{*} \sum_{i=1}^{t} \lambda_{i}^{*}>\alpha\left(\lambda^{*}\right) \quad(j, k) \notin T .
$$

Observe that $\Sigma_{i=1}^{t} \lambda_{i}^{*}$ is always positive since otherwise the $(j, k)-t h$ equations give a contradiction for $(j, k) \in T$.

Clearly, $\alpha\left(\lambda^{*}\right)=\min _{j, k}\left(\overline{\mathrm{f}}_{j, k}\left(w^{*}\right)-w_{j, k}^{*}\right)<0$. The theorem implies that the point $w^{*}$ lies in the set $C(T)$ defined in section 4 but now with respect to $\bar{f}$. Whereas in case of integer labelling the algorithm generates points (simplices) which roughly follow a curve of $C(T)$ defined with respect to the real function $f$, for vector labelling the algorithm generates points which are elements of $C(T)$ defined with respect to a piecewise linear approximation $\bar{f}$ to $\phi$. Observe that $\bar{f}$ changes if the mesh becomes smaller. If $T$ becomes an element not of $z$, then $\Sigma_{i=1}^{t} \lambda_{i}^{*}=1$ and $w^{*}=\sum_{i=1}^{t} \lambda_{i}^{*} w^{i}$ must be a fixed point of $\bar{f}$, cf. corollary 6.5 .6 . Furtiner, for some $\mathrm{T} \in \mathrm{z}$ let $\sigma$ be a generated simplex having two $\mathrm{T}$-complete facets with solutions $\lambda^{*}(1)$ and $\lambda^{*}(2)$ respectively and let $w(1)$ and $w(2)$ be the corresponding solution-points. Then for each point $x=\xi_{w}(1)+(1-\xi) w(2)$ with $0 \leq \xi \leq 1$ we have

$$
\bar{f}_{j, k}(x)-x_{j, k}=\alpha^{\prime} \quad(j, k) \in T
$$

and

$$
\bar{f}_{j, k}(x)-x_{j, k}>\alpha^{\prime} \quad(j, k) \notin T
$$

where $\alpha^{\prime}=\xi \alpha\left(\lambda^{*}(1)\right)+(1-\xi) \alpha\left(\lambda^{*}(2)\right)$. We get the same result if $\sigma$ is a generated simplex of $G(T)$ such that $\sigma$ is $T U\{(i, h)\}$-complete for some $(i, h) \notin T$. Consequently, the algorithm generates a connected chain of line segments lying in $\mathrm{C}(\mathrm{T})$. So, the algorithm actually generates a piecewise linear curve in $S$ starting from $y$ and ending with a fixed point of $\bar{f}$, the latter being an approximate fixed point of $\phi$. If the mesh goes to zero, this point converges to a fixed point of $\phi$. If the mapping $\phi$ is a continuous function, the curve converges to the limiting path mentioned in section 4 . 
It is clear that in case of vector labelling the algorithm has the disposal of more information to decide which direction must be chosen to find an approximate fixed point than in case of integer labelling. So, we may expect that the algorithm converges much faster for vector labelling, in particular if the algorithm is restarted many times to improve the approximation. However, vector labelling requires much storage and involves many computational operations since an $(M+N)$-matrix is adapted by every pivot step and must be stored. Note however that many of the columns are unit columns. It can easily be seen that a unit column remains the same by pivoting so that only the $t$ columns $l\left(y^{i}\right)$ need to be stored. As soon as a unit column is eliminated $t$ increases with one, and when a unit column is reintroduced $t$ decreases with one. Observe that except for Van der Laan and Talman's algorithm discussed in section 4.5 and the algorithm described here no other algorithm has this nice property. To decide whether integer labelling or vector labelling will be used, therefore depends on the computation time of a function evaluation and on the desired accuracy, e.g. if a function evaluation consumes much time it is better to use vector labelling. In the next section we give some computational experience.

6.6. COMPUTATIONAL EXPERIENCE.

As discussed in section 3.2 the computation of an equilibrium strategy of an $\mathrm{N}$-person game, where the $j$-th player has $\mathrm{m}_{j}+1$ strategies, can be considered as the computation of a fixed point of a continuous function $f$ from $S$ into itself. As argued in that section the function $f$ is not very suitable since much information is lost by taking the maximum of 0 and $p^{j}(x)-m_{k}^{j}(x)$ for all $(j, k) \in I(M)$. Therefore we will not apply the algorithm to the function $f$. We relabel the points such that a j-stopping simplex (or a completely labelled simplex) is still a good approximation of an equilibrium strategy. To do so, we formulate the N-person game problem as a nonlinear complementarity problem (cf. Garcia, Lemke and Lüthi [1973]). The equilibrium condition of the $\mathrm{N}$-person game is equivalent to the NLCP

$$
x_{j, k}\left[m_{k}^{j}(x)-v^{j}(x)\right]=0 \text { where } v^{j}(x)=\min _{k \in I_{m_{j}+1}} m_{k}^{j}(x)
$$


such that $x \in S$. Observe that $m_{k}^{j}(x) \geq v^{j}(x)$ for all $(j, k) \in I(M)$ and $\mathrm{x} \in \mathrm{S}$. In case of integer labelling a point $\mathrm{x} \in \mathrm{S}$ receives the label $\ell(\mathrm{x})$ where

$$
\begin{aligned}
\ell(x)=\operatorname{lexicomin}\{(j, k) \mid & x_{j, k}>0 \text { and } m_{k}^{j}(x)-v^{j}(x) \geq m_{h}^{i}(x)-v^{i}(x) \\
& \text { for all } \left.(i, h) \in I(M) \text { such that } x_{i, h}>0\right\} .
\end{aligned}
$$

In case of vector labelling a point $\mathrm{x} \in \mathrm{S}$ receives the $(\mathrm{M}+\mathrm{N})$-vector label $\ell(x)$ where

$$
\begin{aligned}
\ell_{j, k}(x) & =m_{k}^{j}(x)-v^{j}(x)+1 & \text { if } x_{j, k}>0 \\
& =1 & \text { if } x_{j, k}=0 .
\end{aligned}
$$

We applied the algorithm for both labelling rules to two noncooperative three-person games each player having three strategies. For the first game the data of game 2 in Garcia, Lemke and Lüthi [1973] were used. The data of the other game are given in table 6.6.1.

\begin{tabular}{l|ccccccccc} 
& $(1,1)$ & $(1,2)$ & $(1,3)$ & $(2,1)$ & $(2,2)$ & $(2,3)$ & $(3,1)$ & $(3,2)$ & $(3,3)$ \\
\hline$(1,1)$ & 2 & 3 & 4 & 2 & 3 & 3 & 4 & 1 & 5 \\
$(1,2)$ & 1 & 1 & 4 & 3 & 4 & 1 & 6 & 8 & 2 \\
$(1,3)$ & 4 & 7 & 2 & 4 & 5 & 5 & 3 & 6 & 4 \\
$(2,1)$ & 5 & 6 & 7 & 4 & 8 & 9 & 3 & 5 & 1 \\
$(2,2)$ & 1 & 1 & 3 & 3 & 2 & 1 & 2 & 2 & 4 \\
$(2,3)$ & 2 & 3 & 6 & 5 & 3 & 6 & 7 & 5 & 8 \\
$(3,1)$ & 1 & 3 & 5 & 1 & 6 & 2 & 1 & 2 & 4 \\
$(3,2)$ & 2 & 6 & 5 & 3 & 3 & 7 & 8 & 5 & 5 \\
$(3,3)$ & 5 & 2 & 2 & 4 & 6 & 5 & 8 & 1 & 3
\end{tabular}

Table 6.6.1. The number in the $(j, k)$-th row and the $\left(i_{k_{1}}, i_{k_{2}}\right)-$ th column is the loss of player $j$ if he uses his $k$-th pure strategy and if for $h=1,2$ player $k_{h}$ uses his $i_{k_{h}}$-th pure strategy where $k_{1}, k_{2} \neq j$ and $k_{1}<k_{2}$. 
Instead of the standard triangulation matrix $Q_{j}$ on the blockdiagonal of $Q$ we used the matrix $U$ proposed in chapter 5 with- $m_{j}$ on the diagonal. A point outside $S$ received then the label of its projection (per player) on the boundary of $\mathrm{S}$. For both examples the algorithm was started in the barycenter $(1 / 3, \ldots, 1 / 3)^{\top}$ with $a_{j}$ equal to 3 for all $j=1,2,3$, except for the first game when vector labelling was used in which case we have chosen $d_{j}=6$ for all $j$. The factor of incrementation was set equal to two for all applications whereas the final grid size was $384^{-1}$. This is equal to a grid size of $665^{-1}$ in Garcia, Lemke and Lüthi's algorithm. Since their algorithm must start in a corner of $S$, it requires hundred and hundreds of iterations for this grid size. For integer labelling the new starting point was set equal to the barycenter of the j-stopping simplex whereas in case of vector labelling we choose.

$$
y=\sum_{i=1}^{t} \lambda_{i}^{*} w^{i} / \sum_{i=1}^{t} \lambda_{i}^{*}
$$

if $\sigma\left(w^{1}, \ldots, w^{t}\right)$ was the generated completely labelled simplex with solution $\lambda^{*}=\left(\lambda_{1}^{*}, \ldots, \lambda_{t}^{*}\right)$. Note that $\Sigma_{i} \lambda_{i}^{*} \rightarrow 1$ if the mesh goes to zero. The results are shown in the tabels 6.6 .2 and 6.6.3. The accuracy is defined by

$$
\max _{j, k) \in I(M)}\left\{x_{j, k}^{*}\left(m_{k}^{j}\left(x^{*}\right)-v^{j}\left(x^{*}\right)\right)\right\}
$$

where $x^{*}$ is the approximate fixed point.

\begin{tabular}{c|cc|cc} 
Grid size & \multicolumn{2}{|c|}{ Integer labelling } & \multicolumn{2}{c}{ Vector labelling } \\
& Iterations & Accuracy & Iterations & Accuracy \\
\hline 6 & 68 & .025 & 43 & .18 \\
24 & 191 & .075 & 150 & 0 \\
96 & 275 & .012 & - & - \\
384 & 322 & .003 & - & -
\end{tabular}

Table 6.6.2. Game 1. 
The last approximate fixed point was equal to $\mathrm{x}^{*}=(.3904, .2959, .3138 ; .3889, .2970, .3141 ; .9690, .0310,0)$ in case of integer labelling and equal to $x^{*}=u(1,2,2)$ in case of vector labelling.

\begin{tabular}{c|cc|cc} 
Grid size $^{-1}$ & \multicolumn{2}{|c|}{ Integer labelling } & \multicolumn{2}{c}{ Vector labelling } \\
& Iterations & Accuracy & Iterations & Accuracy \\
\hline 6 & 27 & .2 & 40 & .06 \\
24 & 53 & .07 & 55 & .005 \\
96 & 84 & .02 & 71 & .001 \\
384 & 113 & .004 & 87 & .0005
\end{tabular}

Table 6.6.3. Game 2 .

The last approximate fixed point was equal to

$\mathrm{x}^{*}=(.4286, .5714,0 ; 0,1,0 ; .6650, .3350)$ in case of integer labelling and equal to $\mathrm{x}^{*}=(.4284, .5716,0 ; 0,1,0 ; 0, .6667, .3333)$ in case of vector labelling. 
CLASS OF VARIABLE DIMENSION FIXED POINT ALGORITHMS ON $\mathrm{R}^{\mathrm{n}}$

7.1. INTRODUCTION.

Using the ideas of the previous chapter in which an algorithm was developed to compute a fixed point of a continuous function (or u.s.c. mapping) from the product space of unit simplices into itself, we now develop a class of algorithms on $R^{n}$ by considering $R^{n}$ as the product space of lower-dimensional Euclidean spaces. More precisely, let for some $\mathrm{N}$ with $1 \leq N \leq n, m_{1} \ldots, m_{N}$ be given positive integers such that $\Sigma_{i=1}^{N} m_{i}=n$. Then we can write $R^{n}=\pi_{j=1}^{N} R^{m_{j}}$. After triangulating each $R^{\frac{1}{m}}{ }_{j}$ in the standard way we construct a triangulation of $R^{n}$ again defined in relation to an arbitrarily chosen point. This point will be the starting point of the algorithm. Each point is labelled with one of the $n+N$ elements of a set related to the set $I(M)$ defined in the previous chapter. In case of vector labelling a point receives an $(n+N)$-vector label. From the starting point the algorithm generates in the standard way a path of adjacent simplices of the triangulation of variable dimension until some special label set is found. Then an approximate fixed point has been found and the algorithm can be restarted. The steps of the algorithm are almost identical to those of chapter 6. It will appear that from the starting point fast movements can be made in all directions if $\mathrm{N}$ is large. In particular the extreme case $\mathrm{N}=\mathrm{n}$ is of considerable interest since then the efficient $K^{\prime}$ triangulation (cf. also Todd [1978a]) underlies the algorithm. Moreover we will indicate how for $\mathrm{N}=\mathrm{n}$ in case of integer labelling the algorithm can be utilized to approximate a connected set of fixed points. An algorithm closely related to the case $\mathrm{N}=\mathrm{n}$ was independently developed by Reiser [1978a, 1978b] to approximate a solution of the NLCP (see chapter 3). However, his algorithm is based on the $\mathrm{K}$ triangulation and was only presented for integer labelling. For integer labelling we will prove that 
in particular when $\mathrm{N}$ is large, the algorithm can terminate with lowerdimensional simplices without affecting the accuracy. However, for the extreme case $\mathrm{N}=1$ only full-dimensional simplices yielding an approximation exist. The case $\mathrm{N}=1$ is closely related to Van der Laan and Talman's basic algorithm on $\mathrm{R}^{\mathrm{n}}$. Only the labelling rule is different. For vector labelling the algorithm generally terminates with a full-dimensional simplex for any N. For both the integer and vector labelling rule new convergence conditions will be given. It will also appear that Merrill's condition is sufficient for both extreme cases.

In section 2 the triangulation of $R^{n}$ is described for given integers $\mathrm{m}_{1}, \ldots, \mathrm{m}_{\mathrm{N}}$. In section 3 the integer labelling rule is given and it is proved that the algorithm's terminal simplex indeed yields an approximate fixed point. Section 4 presents the algorithm. Also convergence conditions are discussed. The generalization to vector labelling is described in section 5. Section 6 treats the two extreme cases and indicates how the algorithm can be utilized to approximate a connected set of fixed points if $\mathrm{N}$ is equal to $\mathrm{n}$. An application to the Borsuk-Ulam theorem discussed by Todd and Wright [1979] is also presented in section 6. Finally computational experience is given in section 7.

This chapter is based on the work of Van der Laan and Talman [1978b].

7.2. TRIANGULATION OF $R^{n}$ FOR GIVEN POSITIVE INTÉGERS SUMMING UP TO $n$.

Let $\mathrm{m}_{1}, \ldots, \mathrm{m}_{\mathrm{N}}$ be $\mathrm{N}$ positive integers with sum $\mathrm{n}$. Then we can consider $R^{n}$ as the product space of $R_{j}, j=1, \ldots, N$, i.e.

$$
R^{n}=\prod_{j=1}^{N} R^{m_{j}} .
$$

In the same way as described in section 6.2 , we obtain a triangulation of $R^{n}$ by first triangulating each $R^{m_{j}}$ in the standard way. So, let $P_{j}$ be an extended $m_{j} \times\left(m_{j}+1\right)$ triangulation matrix of $R^{m_{j}}$, i.e. let $R^{m_{j}}$ be triangulated according to the $\mathrm{P}_{j} \mathrm{~K}$ triangulation. If $\mathrm{m}_{j}$ is equal to one for some $m_{j}$, we write for ease of notation $P_{j}=[1,-1]$. Furthermore, let $d=\left(d_{1}, \ldots, d_{N}\right)$ be a vector of (positive) grid sizes, and let $D_{j}$ be the diagonal $\mathrm{m}_{j}$-matrix with $d_{j}$ on the diagonal. Then the matrix $\mathrm{D}_{j} \mathrm{P}_{j}$ (or $\mathrm{d}_{j} \mathrm{P}_{j}$ ) induces the $P_{j} K$ triangulation with grid size $d_{j}$. Next, define the 
$n \times(n+N)$ block-matrix $P$ by

$$
\mathrm{P}=\left[\begin{array}{ccccc}
\mathrm{P}_{1} & 0 & \cdot & \cdot & 0 \\
0 & \mathrm{P}_{2} & \cdot & & \cdot \\
\cdot & & \cdot & \cdot \\
\cdot & & & \cdot & \cdot \\
0 & \cdot & \cdot & \cdot & \mathrm{P}_{N}
\end{array}\right] \text {. }
$$

Similarly, let the nxn-matrix $\mathrm{D}$ denote the diagonal matrix whose $\left(k+\Sigma_{i=1}^{j-1} m_{i}\right)$-th diagonal element is equal to $d_{j}$ for $k=1, \ldots, m_{j}$ and $j \in I_{N}$. For $k \in I_{m_{j}+1}$, the $(j, k)-t h$ column of the matrix DP and $P$ is denoted by $\mathrm{Dp}(j, \mathrm{k})$ and $\mathrm{p}(j, \mathrm{k})$ respectively. Also, $e(j, k)$ denotes the $\left(k+\Sigma_{i=1}^{j-1}\left(m_{i}+1\right)\right)-$ th unit vector in $R^{n+N}$ whereas $x_{j, k}$ denotes the $\left(k+\sum_{i=1}^{j-1} m_{i}\right)-t h$ component of $x \in R^{n}$. Following the analysis in chapter 6 , we first "subdivide" $R^{n}$ in regions $A(T)$ for special index sets $T$ such that the rank of the matrix with columns $q(j, k),(j, k) \in T$, is equal to $|T|$. So, let $I(m)$ be defined by

$$
I(m)=\bigcup_{j=1}^{N}\left\{(j, 1), \ldots,\left(j, m_{j}+1\right)\right\},
$$

and let $z$ be the collection of subsets $T$ of $I(m)$ such that for each $j \in I_{n}$ at least one index $(j, k)$ is not in $T$. Then we have the following definition, where $\mathrm{y}$ is an a priori chosen starting point for the algorithm.

DEFINITION 7.2.1. For $T \in \mathrm{Z}$ the region $\mathrm{A}(\mathrm{T})$ is defined by

$$
\begin{gathered}
A(T)=\left\{x \in R^{n} \mid x=y+\sum_{(j, k) \in T} \lambda j, k^{p(j, k) \text { for }}\right. \\
\text { nonnegative numbers } \left.\lambda_{j, k}\right\} .
\end{gathered}
$$

Since the matrix with columns $p(j, k),(j, k) \in T$, has full rank, the (positive) $\lambda_{j, k}$ 's are unique. A point $x \in R^{n}$ is called a proper point of $A(T)$ if $\lambda_{j, k}>0$ for all $(j, k) \in T$. The set of proper points will be again denoted by $\AA(T)$. Observe that int $A(T)=\AA(T)$, independently of the starting point. Moreover, we have the property that $\operatorname{dim} A(T)=|T|, T \in Z$.

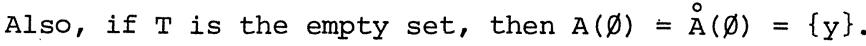


LEMMA 7.2.2. The sets $\AA(T)$ partition $R^{n}, T \in Z$. Moreover, for all $T_{1}$ and $\mathrm{T}_{2}$ in $\mathrm{z}$ holds

$$
A\left(T_{1} \cap T_{2}\right)=A\left(T_{1}\right) \cap A\left(T_{2}\right)
$$

DEFINITION 7.2.3. For $T \in \mathrm{z}$ with $|\mathrm{T}|=\mathrm{t}$ the set $\mathrm{G}(\mathrm{T})$ is the collection of t-simplices $\sigma\left(y^{1}, \pi(T)\right)$ with vertices $y^{1}, \ldots, y^{t+1}$ in $R^{n}$ such that (i) $\quad y^{1}=y+\sum_{(j, k) \in T} d_{j}^{\mu} j, k^{p}(j, k)$ for nonnegative integers $\mu_{j, k^{\prime}}$

(ii) $\pi(T)=\left(\pi_{1}, \ldots, \pi_{t}\right)$ is a permutation of the elements of $T$,

(iii) $y^{i+1}=y^{i}+D p\left(\pi_{i}\right) \quad i=1, \ldots, t$.

Following the proof of theorem 6.2.4 we easily obtain that $G(T)$ is a triangulation of $A(T)$. Next let $z^{1}$ be the subset of $z$ such that $\operatorname{dim} A(T)=n$, then we have the following result. The proof is identical to the combined proofs of theorem 6.2.5 and corollary 6.2.6.

THEOREM 7.2.4. $\mathrm{R}^{\mathrm{n}}$ is triangulated by the union of triangulations $G(T)$ of $A(T), T \in Z^{1}$. Moreover, let $T_{1}$ be a proper subset of $T_{2} \in Z$. Then $G\left(T_{1}\right)$ is the triangulation of $A\left(T_{1}\right)$ induced by $G\left(T_{2}\right)$.

If $\mathrm{N}=1, \mathrm{R}^{\mathrm{n}}$ is triangulated according to the $\mathrm{P}_{1} \mathrm{~K}$ triangulation. Note that in this case each $A(T), T \in Z^{1}$, is triangulated in the same way (see also section 4.5). However, if $N>1$, each region $A(T)$ is differently triangulated. In particular, for $\mathrm{N}=\mathrm{n}$ we obtain the $\mathrm{K}^{\prime}$ triangulation proposed by Todd [1978a, page 162]. He proved that the average directional density of the $\mathrm{K}^{\prime}$ triangulation of $\mathrm{R}^{\mathrm{n}}$ given the starting point $\mathrm{y}$ is of the order of $\mathrm{n}$. As argued by Todd [1973a, page 163] this result must be viewed with caution. Clearly, mesh $K^{\prime}=$ mesh $K$ and $S V\left(K^{\prime}\right)=S V(K)$. The computation of the mesh, the SV measure and the average directional density, defined in chapter 5, of the triangulation is for the case $1<\mathrm{N}<\mathrm{n}$ rather technical and is therefore omitted. Of course, these quantities depend on the structure of the $P_{j}$ matrices and the numbers $m_{j}, j=1, \ldots, N$. Recall that $P_{j}=[1,-1]$ if $m_{j}=1$. In the next section we assign to each (grid)point of $R^{n}$ an integer label being an element of the set $I(m)$. 
7.3. INTEGER LABELLING AND APPROXIMATION.

Suppose we want to compute a fixed point of a continuous function $f$ from $R^{n}$ to $R^{n}$. As mentioned above, we denote the $\left(k+\sum_{i=1}^{j-1} m_{i}\right)-t h$ component of $f(x)=\left(f_{1}(x), \ldots, f_{n}(x)\right)^{\top}$ and $x=\left(x_{1}, \ldots, x_{n}\right)^{\top}$ by $f_{j, k}(x)$ and $x_{j, k}$ respectively $\left(k=1, \ldots, m_{j}\right.$ and $\left.j \in I_{N}\right)$ where $m_{1}, \ldots, m_{N}$ are the given integers with sum $n$. To utilize the triangulation described in the previous section we define the function $g(x)$ from $R^{n}$ to $R^{n+N}$ as follows. For $j=1, \ldots, N$,

and

$$
g_{j, h}(x)=f_{j, h}(x)-x_{j, h} \quad h=1, \ldots, m_{j^{\prime}}
$$

$$
g_{j, m_{j}+1}(x)=-\sum_{h=1}^{m_{j}^{j}} g_{j, h}(x) .
$$

Observe that for $N=1, g_{1, h}=f_{h}(x)-x_{h^{\prime}} h=1, \ldots, n$, and $g_{1, n+1}(x)=-\sum_{i=1}^{n}\left(f_{i}(x)-x_{i}\right)$. Moreover, for $N=n$ we have that $g_{j, 1}(x)=f_{j}(x)-x_{j}$ and $g_{j, 2}(x)=-f_{j}(x)+x_{j}$ for $j=1, \ldots, n$. Based upon the function $g$, the integer labelling rule is defined as follows.

DEFINITION 7.3.1. A point $\mathrm{x} \in \mathrm{R}^{\mathrm{n}}$ receives the integer label $\ell(\mathrm{x}) \in \mathrm{I}(\mathrm{m})$ where

$$
\begin{aligned}
\ell(x)=\operatorname{lexicomin}\{(j, k) \mid & g_{j, k}(x) \geq g_{i, h}(x) \\
& \text { for all indices }(i, h) \in I(m)\} .
\end{aligned}
$$

Since the total number of different labels is $n+N$, we have $N-1$ more labels than in the standard algorithms on $R^{n}$ discussed in chapter 4 . Note that for $N=1$ the labelling is different from the standard labelling. It will be shown below that the standard labelling yieldsiabetter accuracy to an approximate fixed point.

DEFINITION 7.3.2. For $T \in I(m)$ with $|T|=t$, a ( $t-1)$-simplex is $T$-complete if the $t$ vertices of $\sigma$ carry all the labels which are elements of $T$.

DEFINITION 7.3.3. A simplex $\sigma$ is a $j$-stopping simplex, $j \in I_{N^{\prime}}$ if $(j, k)$ is an element of the label set of $\sigma$ for all $\mathrm{k}$. 
Observe that $m_{j} \leq \operatorname{dim} \sigma \leq n$ if $\sigma$ is a j-stopping simplex. Moreover, in the same way as in section 6.3 we have the following corollary.

COROLLARY 7.3.4. A T-complete $\sigma$ is a stopping simplex if and only if $\mathrm{T} \notin \mathrm{z}$.

In the next theorem we prove that a j-stopping simplex yields a good approximation of a fixed point of $\mathrm{f}$.

THEOREM 7.3.5. Let $\varepsilon, \delta>0$ be such that $\max _{\mathrm{s}}\left|\mathrm{x}_{\mathrm{s}}-\mathrm{y}_{\mathrm{S}}\right| \leq \delta$ and $\max _{S}\left|f_{S}(x)-f_{S}(y)\right|<\varepsilon$ for all $x$ and $y$ in a j-stopping simplex $\sigma$. Then for any $\mathrm{x} \in \sigma$

$$
\max _{s \in I_{n}}\left|f_{s}(x)-x_{s}\right|<\frac{1}{2}(n+1)(\varepsilon+\delta) . \quad \text { if } N=1
$$

and

$$
\max _{s \in I_{n}}\left|f_{s}(x)-x_{s}\right|<\left\{1+\frac{1}{2}\left(m_{j}+1\right) \max _{i \neq j} m_{i}\right\}(\varepsilon+\delta) \quad \text { if } N \geq 2 \text {. }
$$

PROOF. Let $w(k)$ be the vertex of $\sigma$ such that $\ell(w(k))=(j, k), k=1, \ldots, m_{j}+1$. Since $\Sigma_{\mathrm{k}=1}^{\mathrm{m}_{\mathrm{j}}+1} \mathrm{~g}_{\mathrm{j}, \mathrm{k}}(\mathrm{y})=0$, it follows from definition 7.3 .1 that

$$
g_{j, k}(w(k)) \geq 0 \quad \text { for } k=1, \ldots, m_{j}+1
$$

Hence, for $\mathrm{k}=1, \ldots, \mathrm{m}_{\mathrm{j}}$ and for an arbitrarily chosen point $\mathrm{x} \epsilon \sigma$,

$$
\begin{aligned}
f_{j, k}(x)-x_{j, k} & =\left(f_{j, k}(x)-f_{j, k}(w(k))\right)+g_{j, k}(w(k)) \\
& +\left(w_{j, k}(k)-x_{j, k}\right)>-(\varepsilon+\delta) .
\end{aligned}
$$

Moreover, for $\mathrm{k}=1, \ldots, \mathrm{m}_{\mathrm{j}}$

$$
g_{j, k}\left(w\left(m_{j}+1\right)\right)<g_{j, m_{j}+1}\left(w\left(m_{j}+1\right)\right)=-\sum_{h=1}^{m} g_{j, h}\left(w\left(m_{j}+1\right)\right),
$$

and so

$$
g_{j, k}\left(w\left(m_{j}+1\right)\right)<-\frac{1}{2} \sum_{\substack{h=1 \\ h \neq k}}^{m} g_{j, h}\left(w\left(m_{j}+1\right)\right)<\frac{1}{2}\left(m_{j}-1\right)(\varepsilon+\delta) .
$$


Therefore,

$$
\begin{array}{r}
f_{j, k}(x)-x_{j, k}<g_{j, k}\left(w\left(m_{j}+1\right)\right)+\varepsilon+\delta<\frac{1}{2}\left(m_{j}+1\right)(\varepsilon+\delta) \\
\text { for } k=1, \ldots, m_{j} .
\end{array}
$$

Consequently,

(7.3.1) $\max _{k \in I_{m_{j}}}\left|f_{j, k}(x)-x_{j, k}\right|<\frac{1}{2}\left(m_{j}+1\right)(\varepsilon+\delta)$.

For $N=1\left(m_{1}=n\right)$ we obtain

$$
\max _{s \in I_{n}}\left|f_{s}(x)-x_{s}\right|<\frac{1}{2}(n+1)(\varepsilon+\delta),
$$

which proves the first part of the theorem.

For $N \geq 2$ and $i \neq j$ we have that for $h=1, \ldots, m_{i}+1$

$$
g_{i, h}(w(1)) \leq g_{j, 1}(w(1))<\frac{1}{2}\left(m_{j}+1\right)(\varepsilon+\delta) .
$$

Hence, for $h=1, \ldots, m_{i}$,

$$
g_{i, h}(w(1))=-\sum_{\substack{k=1 \\ k \neq h}}^{m_{i}+1} g_{i, k}(w(1))>-\frac{1}{2} m_{i}\left(m_{j}+1\right)(\varepsilon+\delta),
$$

and so

$$
\left\{1+\frac{1}{2}\left(m_{j}+1\right)\right\}(\varepsilon+\delta)>f_{i, h}(x)-x_{i, h}>-\left\{1+\frac{1}{2} m_{i}\left(m_{j}+1\right)\right\}(\varepsilon+\delta) .
$$

Combining the last two inequalities with inequality (7.3.1) we obtain

$$
\max _{s \in I_{n}}\left|f_{s}(x)-x_{s}\right|<\left\{1+\frac{1}{2}\left(m_{j}+1\right) \max _{i \neq j} m_{i}\right\}(\varepsilon+\delta)
$$


COROLLARY 7.3.6. For $\mathrm{N}=\mathrm{n}\left(\mathrm{m}_{j}=1\right)$ we have for every $\mathrm{x}$ in a $j$-stopping simplex

$$
\max _{s \in I_{n}}\left|f_{s}(x)-x_{S}\right|<2(\varepsilon+\delta) .
$$

Observe that for all $\mathrm{N}$ the inaccuracy of the approximation is higher than the inaccuracy obtained for the standard labelling rule, even in case $\mathrm{N}=1$. This is caused by the fact that label $\mathrm{n}+1$ is assigned as soon as $-\sum_{s=1}^{n}\left(f_{s}(x)-x_{s}\right)$ is larger than $f_{j}(x)-x_{j}$ for all $j$, whereas in the basic algorithm label $n+1$ is only assigned if $f_{s}(x)-x_{s}<0$ for all s. Hence, a completely labelled simplex gives a better approximation in case of standard labelling. Note that for the other extreme case $N=n$, the inaccuracy is two times higher than in case of the standard labelling rule (for $\mathrm{N}=1$ ). In section 6 we propose a labelling rule for the case $\mathrm{N}=\mathrm{n}$ which will improve the accuracy. Finally, we remark that the accuracy of the approximation obtained from a j-stopping simplex $\sigma$ depends not on the number of vertices of $\sigma$. Of course, it depends on $m_{j}$. For instance, if $\mathrm{n}=10, \mathrm{~N}=2, \mathrm{~m}_{1}=1$ and $\mathrm{m}_{2}=9$, the inaccuracy is $10(\varepsilon+\delta)$ if a 1-stopping simplex $\sigma\left(w^{1}, \ldots, w^{t}\right)$ is found $(2 \leq t \leq 11)$ and the inaccuracy is $6(\varepsilon+\delta)$ if a 2-stopping simplex $\sigma\left(w^{1}, \ldots, w^{t}\right)$ is found $(10 \leq t \leq 11)$.

7.4. THE VARIABLE DIMENSION ALGORITHM ON $\mathrm{R}^{\mathrm{n}}$.

Let $R^{n}$ be triangulated for given $y$ as described in section 2 and let each point $\mathrm{x}$ in $\mathrm{R}^{\mathrm{n}}$ be labelled according to definition 7.3 .1 for given $m_{1}, \ldots, m_{N}$ with sum $n$. To generate for some $j \in I_{N}$ a j-stopping simplex of the triangulation, we apply the algorithm described in section 6.4 by performing the steps $0-4$, starting with the arbitrarily chosen point $y$. Of course, columns of the matrix $Q$ must be changed into the same columns of the matrix P. Hence, performing the algorithm, a path of adjacent simplices $\sigma$ of the triangulation of variable dimension is generated such that when $\sigma$ is a generated t-simplex $\sigma\left(y^{1}, \pi(T)\right)$ of $G(T)$, $T \in \mathrm{Z}$, either $\sigma$ has two $\mathrm{T}$-complete facets or $\sigma$ is $(T \cup\{(j, k)\})$-complete for some $(j, k) \notin T$. In the latter case the algorithm terminates with the $j$-stopping simplex $\sigma\left(\mathrm{Y}^{1}, \pi(\mathrm{T})\right)$ if $\mathrm{T} \cup\{(\mathrm{j}, \mathrm{k})\} \notin \mathrm{z}$, whereas the dimension is increased if $\sigma$ was entered via the T-complete facet of $\sigma$, and the dimension is decreased if $\sigma$ was entered from the $(t+1)$-simplex 
$\bar{\sigma}\left(y^{1},(\pi(T),(j, k))\right)$. In the latter case the last vertex of $\bar{\sigma}$ is deleted and the vertex having label $(j, k)$ is removed. In both cases $\sigma$ has exactly two adjacent simplices with one of the just mentioned properties. If $\sigma$ has two T-complete facets, $\sigma$ was entered via one of them whereas the algorithm leaves $\sigma$ via the other one. Also in this case $\sigma$ has exactly two edjacent simplices with one of the properties. However, only the zero-dimensional starting simplex $\{y\}$ has just one adjacent simplex $\sigma\left(y^{1}, \pi(T)\right)$ of $G(T)$ which has either two T-complete facets or is $(T \cup\{(j, k)\})$-complete, viz. for $\mathrm{y}^{1}=\mathrm{y}$ and $\mathrm{T}=\{l(\mathrm{y})\}$. So, the algorithm can never return in a previously generated simplex. Consequently, either the algorithm terminates with a stopping simplex, or a path of simplices going to infinity is generated. The next theorem gives a condition to guarantee convergence.

THEOREM 7.4.1. Let $f$ be a continuous function from $R^{n}$ to $R^{n}$ and let $y$ be the starting point. Assume there exist numbers $\mu>\max _{\mathbf{S}}\left|\mathrm{y}_{\mathbf{S}}\right|$ and $\varepsilon>0$ such that for any $x \in B(\mu)=\left\{z \in R^{n}\left|\max _{S}\right| z_{S} \mid=\mu\right\}$ at least one of the following conditions holds:

(i) there is an index s with $x_{S}>y_{S}$ such that $f_{S}(x)-x_{S}+\varepsilon<g_{r, h}(x)$ for some index $(r, h) \in I(m)$,

(ii) there is an index $j$ with $\sum_{h=1}^{m}\left(y_{j, h}-x_{j, h}\right)>0$ such that $g_{j, m_{j}+1}(x)+\varepsilon<g_{r, h}(x)$ for some index $(r, h) \in I(m)$.

Then the algorithm terminates within a finite number of iterations if $P_{j}$ is the $m_{j} \times\left(m_{j}+1\right)$ triangulation matrix corresponding to an $\left(\alpha_{j}, \beta_{j}\right)-$ triangulation of $R^{m}$ (with $\beta_{j} \leq 0$ and $\alpha_{j}+\left(m_{j}-1\right) \beta_{j}>0$ ) and if the mesh of the triangulation is small enough.

PROOF. Let the grid be so fine that

$$
\max _{i \in I_{n}}\left|f_{i}\left(x^{1}\right)-x_{i}^{1}-f_{i}\left(x^{2}\right)+x_{i}^{2}\right|<\frac{1}{2} \varepsilon \min _{p \in I_{N}} m_{p}^{-1}
$$

for every two points $\mathrm{x}^{1}$ and $\mathrm{x}^{2}$ in a simplex $\sigma$ of the triangulation meeting $B(\mu)$. Let for some $T \in Z, \sigma\left(y^{1}, \pi(T)\right)$ be a simplex of $G(T)$ such that 
$\sigma \cap B(\mu) \neq \varnothing$. We will prove that $\sigma$ has no $\mathrm{T}$-complete facets and therefore cannot be generated by the algorithm. Let $x$ be an arbitrarily chosen point in the intersection of $\sigma$ and $B(\mu)$. If $(i)$ holds for some $s$, then $(j, k) \in T$, where $s=k+\sum_{i=1}^{j-1} m_{i}$, since $x_{s}>y_{s}$. Moreover, $g_{r, h}(x)>f_{\underline{s}_{1}}(x)-x_{s}+\varepsilon$ for at least one index $(r, h)$. Since $g_{r, h}(x)<g_{r, h}\left(y^{i}\right)+\frac{1}{2} \varepsilon \min _{p} m_{p}^{-1} m_{r}$ for all vertices $y^{i}$ of $\sigma$, it follows that for all $i$

$$
\begin{aligned}
f_{s}\left(y^{i}\right)-y_{s}^{i} & <f_{s}(x)-x_{s}+\frac{1}{2} \varepsilon \min _{p} m_{p}^{-1} \\
& <g_{r, h}(x)-\varepsilon+\frac{1}{2} \varepsilon \min _{p} m_{p}^{-1} \\
& <g_{r, h}\left(y^{i}\right)-\varepsilon+\frac{1}{2} \varepsilon \min _{p} m_{p}^{-1}\left(m_{r}+1\right) \\
& \leq g_{r, h}\left(y^{i}\right) .
\end{aligned}
$$

Consequently, no vertex of $\sigma\left(y^{1}, \pi(T)\right)$ carries label $(j, k)$ which implies that $\sigma$ has no $T$-complete facets since $(j, k) \in T$.

On the other hand if (ii) holds for some index $j$, then $\left(j, m_{j}+1\right) \in T$ and $g_{r, h}(x)>g_{j, m_{j}+1}(x)+\varepsilon$ for at least one index $(r, h)$.

Using the same arguments as above we obtain that for all $i$

$$
\begin{aligned}
g_{j, m_{j}+1}\left(y^{i}\right) & <g_{j, m_{j}+1}(x)+\frac{1}{2} \varepsilon \min _{p} m_{p}^{-1} m_{j} \\
& <g_{r, h}(x)-\varepsilon+\frac{1}{2} \varepsilon \min _{p} m_{p}^{-1} m_{j} \\
& <g_{r, h}\left(y^{i}\right)-\varepsilon+\frac{1}{r} \varepsilon \min _{p} m_{p}^{-1}\left(m_{j}+m_{r}\right) \\
& \leq g_{r, h}\left(y^{i}\right) .
\end{aligned}
$$

Hence, no vertex of $\sigma$ carries label $\left(j, m_{j}+1\right)$ which again implies that $\sigma$ has no T-complete facets since $\left(j, \mathrm{~m}_{j}+1\right) \in \mathrm{T}$. Therefore, starting with the zero-dimensional simplex $\{y\}$ no simplex meeting $B(\mu)$ can be generated by the algorithm. This proves the theorem since the number of simplices in conv $(B(\mu))$ is finite.

Note that for $N=n$ the condition of the theorem can be simplified. 
COROLLARRY 7.4.2. Let $f$ be a continuous function from $R^{n}$ to $R^{n}$ and let $y$ be the starting point. Assume there exist numbers $\mu>\max _{j}\left|y_{j}\right|$ and $\varepsilon>0$ such that for any $\mathrm{x} \in \mathrm{B}(\mu)$ there exists an index $\mathrm{s}$ with $\mathrm{x}_{\mathrm{s}} \neq \mathrm{y}_{\mathrm{s}}$ and

$$
\left(f_{s}(x)-x_{s}\right) \operatorname{sgn}\left(x_{s}-y_{s}\right)+\varepsilon<\left|f_{j}(x)-x_{j}\right|
$$

for at least one index $j \in I_{N}$. Then the algorithm terminates if the grid size is fine enough and if $\mathrm{N}=\mathrm{n}$.

Remark that theorem 7.4.1 is not always valid if other triangulation matrices $\mathrm{P}_{j}$ underly the algorithm. Conditions for other matrices can easily be derived. They must state that for $\mathrm{x} \in \mathrm{A}(\mathrm{T})$ "far enough away" some label $(j, k) \in T$ does not occur in the neighbourhood of $x$.

We now give a condition which is independent of the starting point and the grid size vector.

THEOREM 7.4.3. Let $f$ be a continuous function from $R^{n}$ to $R^{n}$. Assume there exists a $\mu>0$ such that $x_{j, k}>\mu$ implies

$$
f_{j, k}(x)-x_{j, k}<g_{r, h}(x) \text { for some index }(r, h) \text {, }
$$

and such that $x_{j, k}<-\mu$ implies

$$
g_{j, m_{j}+1}(x)<g_{r, h}(x) \quad \text { for some index }(r, h) \text {. }
$$

Then the algorithm converges for every starting point and for every vector of grid sizes if $P_{j}$ is of the $\left(\alpha_{j}, \beta_{j}\right)$-form.

PROOF. Let mesh $\mathrm{G}$ be equal to $\delta$ and let $\mathrm{y}$ be an arbitrarily chosen starting point in $\mathrm{R}^{\mathrm{n}}$. Moreover, let $\mu^{\prime}>\max _{\mathrm{S}}\left|\mathrm{y}_{\mathrm{S}}\right|+\mu+\delta$, and define the set $\bar{B}\left(y, \mu^{\prime}\right)$ by

$$
\bar{B}\left(y, \mu^{\prime}\right)=\left\{x \in R^{n} \mid-\mu_{j}^{\prime} \leq x_{j, k}-y_{j, k} \leq \mu^{\prime} \text { for all }(j, k) \in I(m)\right\}
$$

where $\mu_{j}^{\prime}=\mu^{\prime} \max \left(1, \mathrm{~m}_{j}-1\right)$.

For some $T \in z$ let $\sigma\left(y^{1}, \pi(T)\right)$ be a simplex of the triangulation $G(T)$ of $A(T)$ such that $\sigma \cap$ bd $\bar{B}\left(y, \mu^{\prime}\right) \neq \varnothing$. We will prove that $\sigma$ has no T-complete facets. Let $x \in \sigma \cap$ bd $\bar{B}\left(y, \mu^{\prime}\right)$, then there is some index $(j, k)$ such that 
either $x_{j, k}-y_{j, k}=\mu^{\prime}$ or $x_{j, k}-y_{j, k}=-\mu_{j}^{\prime} \cdot$ If $x_{j, k}-y_{j, k}=\mu^{\prime}$, then since $x_{j, k}>y_{j, k}(j, k) \in T$ since $x_{j, k}>y_{j, k}$ and $x_{j, k}>\mu^{\prime}-\max _{s}\left|y_{s}\right|>\mu+\delta$ Conseruently, $r_{j, k}^{i}>\mu$ for all vertices $y^{\perp}$ of $\sigma$, and so for each $i$ there exists an index $(r, h)$ such that

$$
f_{j, k}\left(y^{i}\right)-y_{j, k}^{i}<g_{r, h}\left(y^{i}\right) .
$$

Hence, no vertex of $\sigma$ carries label $(j, k)$. Therefore, $\sigma$ has no T-complete facets. Observe that $(r, h)$ need not to be the same for all $y^{i}$. Suppose now that $x_{j, k}-y_{j, k}=-\mu_{j}^{\prime}$. We first show that this implies $\left(j, m_{j}+1\right) \in T$. For $m_{j}=1$ the proof is obvious. If $m_{j}>1$ then, because of the structure of $P_{j}$, there exists an index $(j, h)$ when $\left(j, m_{j}+1\right) \notin T$ such that

$$
x_{j, h}-y_{j, h}>\left(1-\frac{m_{j}-2}{m_{j}-1}\right)\left(y_{j, k}-x_{j, k}\right)=\mu_{j}\left(m_{j}-1\right)^{-1}=\mu^{\prime},
$$

which contradicts the fact that $x_{i, h}-y_{i, h} \leq \mu^{\prime}$ for all $(i, h)$. Hence $\left(j, m_{j}+1\right) \in T$. Moreover, $x_{j, k}-y_{j, k}=-\mu_{j}^{\prime}$ implies $x_{j, k}<\max _{s}\left|y_{s}\right|-\mu^{\prime}<-\mu-\delta$, and so $y_{j, k}^{i}<-\mu$ for all vertices $y^{i}$ of $\sigma$. Hence, for all $i$ there is an index $(r, h)$ such that

$$
g_{j, m_{j}+1}\left(y^{i}\right)<g_{r, h}\left(y^{i}\right)
$$

which implies that no vertex of $\sigma$ carries label $\left(j, m_{j}+1\right)$. Consequently, $\sigma$ has no T-completely facets. So, no simplices outside $\bar{B}\left(Y, \mu^{\prime}\right)$ can be generated by the algorithm and hence, since the number of simplices meeting $\bar{B}\left(y, \mu^{\prime}\right)$ is finite, the algorithm converges.

COROLLARY 7.4.4. Let $f$ be a continuous function from $R^{n}$ to $R^{n}$. Assume there exists a $\mu>0$ such that $\left|\mathbf{x}_{\mathbf{S}}\right|>\mu$ implies

$$
\operatorname{sgn} x_{s}\left(f_{s}(x)-x_{s}\right)<\max _{i \in I_{n}}\left|f_{i}(x)-x_{i}\right|
$$


Then for $\mathrm{N}=\mathrm{n}$ the algorithm converges always.

Observe that for the other extreme case $N=1$ the condition differs from the convergence-condition stated by Van der Laan [1980] for the standard labelling rule. Recall that the standard labelling rule should be preferred since it performs better. Convergence-conditions for other triangulation matrices can be easily derived.

Concerning the local path followed by the sequence of generated simplices we can make the same remarks as made in section 6.4. Note, however, that the path is now defined with respect to the function $g$ and not with respect to $\mathrm{f}$. We do not further discuss this matter and continue with vector labelling.

\subsection{VECTOR LABELLING.}

To apply the algorithm described in the previous section for the computation of a fixed point of an u.s.c. mapping $\phi$ from $R^{n}$ to the set of nonempty, convex subsets of $\mathrm{R}^{\mathrm{n}}$, we consider again the triangulation of $\mathrm{R}^{\mathrm{n}}$ as defined in section 2 for given positive integers $\mathrm{m}_{1}, \ldots, \mathrm{m}_{\mathrm{N}}$ with sum $\mathrm{n}$ and some starting point $\mathrm{y}$. Now each point $\mathrm{x}$ in $\mathrm{R}^{\mathrm{N}}$ receives the $(n+N)$-vector label $l(x)=g(x)+e$ where $g(x)$ is defined as in section 3 with respect to a piecewise linear approximation $\bar{f}$ to $\phi$.

DEFINITION 7.5.1. A $(t-1)$-simplex $\sigma\left(w^{1}, \ldots, w^{t}\right)$ is T-complete, for some $T \subset I(m)$ with $|T|=t$, if the system of $n+N$ linear equations

$$
\sum_{i=1}^{t} \lambda_{i} l\left(w^{i}\right)+\sum_{(j, k) \notin T} \mu_{j, k} e(j, k)=e
$$

has a nonnegative solution $\lambda_{i^{\prime}}^{*} i=1, \ldots, t$, and $\mu_{j, k^{\prime}}^{*}(j, k) \notin T$.

Clearly, if $\sigma\left(w^{1}, \ldots, w^{t}\right)$ is a $T$-complete simplex, then

$$
(n+N) \sum_{i=1}^{t} \lambda_{i}^{*}+\sum_{(j, k) \notin T} \mu_{j, k}^{*}=n+N
$$

and with $\mu_{j, h}^{*}=0$ when $(j, h) \in T$ 
(7.5.1) $\quad\left(1+m_{j}\right) \sum_{i=1}^{t} \lambda_{i}^{*}+\sum_{k=1}^{m_{j}+1} \mu_{j, k}^{*}=1+m_{j} \quad j=1, \ldots, N$.

DEFINITION 7.5.2. A T-complete simplex is a $j$-stopping face if $(j, k) \in T$ for all $k=1, \ldots, m_{j}+1$.

DEFINITION 7.5.3. A T-complete simplex is completely labelled if $\mu_{j, k}^{*}=0$ for all $(j, k)$.

Obviously, if a T-complete simplex is a j-stopping face, it is also. completely labelled. In the next theorem, which can be easily generalized to u.s.c. mappings, we state that for a continuous function $\phi=f a$ completely labelled simplex yields an approximate fixed point.

COROLLARY 7.5.4. Let $\varepsilon, \delta>0$ be such that $\max _{s}\left|x_{s}-y_{s}\right| \leq \delta$ and $\max _{S}\left|f_{S}(x)-f_{S}(y)\right|<\varepsilon$ for all $x$ and $y$ in a completely labelled $t$-simplex $\sigma\left(y^{1}, \ldots, y^{t+1}\right)$. Then, with $x^{*}=\Sigma_{i} \lambda_{i}^{*} y^{i}$, we have that

$$
\max _{s \in I_{n}}\left|f_{s}\left(x^{*}\right)-x_{s}^{*}\right|<\varepsilon
$$

Moreover, $x^{*}$ is a fixed point of $\overline{\mathrm{f}}$.

Observe, that equality (7.5.1) implies $\Sigma_{i} \lambda_{i}^{*}=1$ and that the accuracy of the approximation is the same as that of a completely labelled simplex for the standard labelling rule $(N=1)$. So, the $\mathrm{m}_{j}$ 's have no influence on the inaccuracy.

To generate a completely labelled simplex, the algorithm operates as described in section 6.5 starting with the arbitrarily chosen zero-dimensional simplex $\{y\}$ and the system of linear equations

$$
\Sigma_{(j, k)} \mu_{j, k} e(j, k)=e
$$

where the sum is again over all indices $(j, k)$. Following now the steps discussed in section 6.5 (only adapted for the matrix P) a path of adjacent simplices of the triangulation of variable dimension is generated such that a generated t-simplex $\sigma$ either has two $\mathrm{T}$-complete facets or is 
( $T \cup\{(j, k)\})$-complete for some $(j, k) \notin T$, with $T \in Z$. In both cases $\sigma$ is a simplex of the form $\sigma\left(\mathrm{Y}^{1}, \pi(T)\right)$ of the triangulation $G(T)$ of $A(T)$. As soon as by a pivot step all $\mu_{i, h}^{*}$ 's become equal to zero, the algorithm terminates. This will be always the case if $\sigma$ is $(T \cup\{(j, k)\})$-complete for some $(j, k) \notin T$ such that $T U\{(j, k)\} \notin z$. So, using the standard arguments, the algorithm either terminates with a completely labelled simplex or it generates a path going to infinity. To guarantee that the algorithm converges we can give analoguous conditions as in the case of integer labelling.

THEOREM 7.5.5. Let $\mathrm{f}$ be a continuous function from $\mathrm{R}^{\mathrm{n}}$ to $\mathrm{R}^{\mathrm{n}}$ and let $\mathrm{y}$ be the starting point. Then the algorithm terminates under the same conditions as formulated in theorem 7.4.1.

PROOF. Jet the grid be so fine that

$$
\max _{i \in I_{n}}\left|f_{i}\left(x^{1}\right)-x_{i}^{1}-f_{i}\left(x^{2}\right)+x_{i}^{2}\right|<\frac{1}{2} \varepsilon \min _{D \in I_{N}} m_{p}^{-1}
$$

for every two points $x^{1}$ and $x^{2}$ in the same simplex $\sigma$ in $G$ meeting $B(\mu)$. Let $\mathrm{x}$ be an element of $\sigma\left(\mathrm{y}^{1}, \pi(T)\right) \cap B(\mu) \neq \varnothing$ for some $T \in \mathrm{z}$. If (i) holds, then there is an index $(r, h)$ such that for all vertices $y^{i}$ of $\sigma$

$$
f_{s}\left(y^{i}\right)-y_{s}^{i}<g_{r, h}\left(y^{i}\right)
$$

as follows from the proof of theorem 7.4.1. Since $y_{S}>v_{S^{\prime}}$ the corresponding unit column $e(j, k)$ has already been eliminated so that the $(j, k)-$ th equation of the linear system with respect to $\sigma$ is equal to

$$
\sum_{i=1}^{t+1} \lambda_{i}\left(f_{s}\left(y^{i}\right)-y_{s}^{i}+1\right)=1
$$

Clearly, at least one $\lambda_{i}$ is positive. Subtracting now the $(r, h)-t h$ equation gives

$$
\sum_{i=1}^{t+1} \lambda_{i}\left\{f_{s}\left(y^{i}\right)-y_{s}^{i}-g_{r, h}\left(y^{i}\right)\right\}-\mu_{r, h}=0
$$

where $\mu_{r, h}=0$ if $(r, h) \in T$. Since for all $i$ the term between the main brackets is negative and since $\mu_{r, h}$ is nonnegative, the system has no feasible solution. Consequently $\sigma\left(\mathrm{Y}^{1}, \pi(\mathrm{T})\right)$ has no T-complete facets. 
If on the other hand (ii) holds, then also by the proof of theorem 7.4.1 there exists an index $(r, h)$ such that for all vertices $y^{i}$ of $\sigma$

$$
g_{j, m_{j}+1}\left(y^{i}\right)<g_{r, h}\left(y^{i}\right) .
$$

Moreover, since $\Sigma_{k} y_{j, k}>\Sigma_{k} x_{j, k^{\prime}}$ the $\left(j, m_{j}+1\right)-t h$ unit column has already been eliminated so that the corresponding equation is equal to

$$
\sum_{i=1}^{t} \lambda_{i}\left\{g_{j, m_{j}+1}\left(y^{i}\right)+1\right\}=1,
$$

which again implies that $\sigma$ has no T-complete facets. Hence, no simplices $\sigma\left(y^{1}, \pi(T)\right)$ meeting $B(\mu)$ are generated by the algorithm which proves that the algorithm converges since the number of simplices within the convex hull of $B(\mu)$ is finite.

If $\mathrm{N}=\mathrm{n}$ we obtain the analogon of corollary 7.4.2. Of course, the theorem can be easily generalized for other triangulation matrices. Finally, we give a condition which is independent of the starting point and the grid size. However the condition differs somewhat from the one stated in theorem 7.4.3. This is caused by the fact that in case of integer labelling the leaving vertex is determined only, by the label of the new vertex. For vector labelling however, all labels together determine which vertex has to be removed.

THEOREM 7.5.6. Let $f$ be a continuous function from $R^{n}$ to $R^{n}$. Assume there exists a $\mu>0$ such that $x_{j, k}>\mu$ implies

$$
f_{j, k}(x)-x_{j, k}<0
$$

and $x_{j, k}<-\mu$ implies

$$
\sum_{h=1}^{m}\left(f_{j, h}(x)-x_{j, h}\right)>0
$$

Then the algorithm converges for every starting point and for every vector of grid sizes if $P_{j}$ is of the $\left(\alpha_{j}, \beta_{j}\right)$-form. 
PROOF. Let $\delta$ be the mesh of the triangulation and let $y$ be the arbitrarily chosen starting point. Let $\mu^{\prime}$ and $\bar{B}\left(y, \mu^{\prime}\right)$ be defined as in the proof of theorem 7.4.3. Furthermore, take for some $T \in Z$ a simplex $\sigma\left(y^{1}, \pi(T)\right)$ of $G(T)$ such that $\sigma \cap \bar{B}\left(y, \mu^{\prime}\right) \neq \varnothing$. Let $x$ be an arbitrary point in this intersection. If $x_{j, k}-y_{j, k_{i}}=\mu^{\prime}$ for some $(j, k)$ this index must be an element of $T$, whereas $f_{j, k}\left(y^{i}\right)-y_{j, k}^{i}<0$ for all vertices $y^{i}$ of $\sigma$, as follows from the proof of theorem 7.4.3. Since $(j, k) \in T$ the $(j, k)-t h$ equation of the system of linear equations with respect to $\sigma$ is equal to

$$
\sum_{i=1}^{t+1} \lambda_{i}\left(f_{j, k}\left(y^{i}\right)-y_{j, k}^{i}+1\right)=1
$$

which implies that $\Sigma_{i} \lambda_{i}>1$. According to equality (7.5.1), if $\Sigma_{i} \lambda_{i}>1$ the system has no feasible solution. Hence $\sigma$ has no T-complete facets. Suppose now that $x_{j, k}-y_{j, k}=-\mu_{j}^{\prime}$. Then in the same way as in the proof of theorem 7.4 .3 we obtain that $\left(j, m_{j}+1\right) \in T$ and $g_{j, m_{j}+1}\left(y^{i}\right)<0$ for all
vertices $y^{i}$ of $\sigma$. Hence,

$$
\sum_{i=1}^{t+1} \lambda_{i}\left(g_{j, m_{j}+1}\left(y^{i}\right)+1\right)=1
$$

implying that $\Sigma_{i} \lambda_{i}>1$. From $(7.5 .1)$ we obtain again that $\sigma$ has no T-complete facets which proves the theorem.

The same condition for all $f(x) \in \phi(x), x \in R^{n}$, 'quarantees convergence for an u.s.c. mapping $\phi$. Analoguous conditions for other triangulation matrices $P_{j}$ can be again easily derived. These conditions must be such that a simplex $\sigma\left(y^{1}, \pi(T)\right)$ of the triangulation $G(T)$ of $A(T)$ has no T-complete facets when this simplex is "far away". For $\mathrm{N}=\mathrm{n}$ we have the following corollary.

COROLLARY 7.5.7. Let $f$ be a continuous function from $R^{n}$ to $R^{n}$. Assume there exists a $\mu>0$ such that $\left|\mathbf{x}_{\mathrm{S}}\right|>\mu$ implies

$$
\left(f_{s}(x)-x_{s}\right) \operatorname{sgn} x_{S}<0 .
$$

Then the algorithm converges for every starting point $y$ and for every vector d of grid sizes. 
Note that this is a "symmetric" condition and can not be applied if $\mathrm{N}<\mathrm{n}$. Moreover, the condition is easy to verify. In the next section we will prove that Merrill's condition is sufficient for both $N=1$ and $N=n$. For other values of $\mathrm{N}$ it is not clear whether Merrill's condition can be used. However, the intermediate cases $(1<\mathrm{N}<\mathrm{n})$ are not so interesting except when the function is linear in some components or separable (see Kojima $[1978 a, 1978 b])$. Therefore we pay no further attention to the case $\mathrm{S} \quad 1<\mathrm{N}<\mathrm{n}$.

7.6. THE TWO EXTREME CASES $\mathrm{N}=1$ AND $\mathrm{N}=\mathrm{n}$.

As mentioned above the case $\mathrm{N}=1$ gives an algorithm which is almost identical to Van der Laan and Talman's basic algorithm discussed in section 4.5. The only difference is the labelling rule. It is not clear which of the two vector labelling rules is better. Recall that in case of integer labelling the standard rule is better. Concerning the convergence of the algorithm one can easily construct examples such that the algorithm converges for the standard labelling rule but diverges for the rule defined in this chapter and reversely. However, Merrill's condition is sufficient for both (vector) labelling rules if the $(\alpha, \beta)$ triangulation underlies the algorithm for $\beta<0$. The proof for the standard labelling rule can be found in Van der Laan [1980, chapter 6] and Van der Laan and Talman [1980c]. For the labelling rule of this chapter the result is proved in the next theorem where for ease of notation the index $(1, h)$ is denoted by $h, h \in I_{n+1}$. Recall that $N=1$.

THEOREM 7.6.1. Suppose that for some $\delta>0$ there exist $w \in R^{n}$ and $\rho>0$ such that for all $x_{i}, z \in R^{n}$ with $\max _{i}\left|x_{i}\right|>\rho$ and $\max _{i}\left|x_{i}-z_{i}\right|<\delta$ holds

$$
\left.(f(x)-x)^{\top}(w-z)>0 \quad \text { (for all } f(x) \in \phi(x)\right) \text {. }
$$

Then the algorithm converges if the mesh of the triangulation is small enough and if the $(n+\sqrt{n+1})$-triangulation underlies the algorithm.

PROOF. Let the mesh of the triangulation be less than $\delta$ and let $y$ be the starting point of the algorithm. Define $\rho^{\prime}$ by 


$$
\begin{array}{r}
\rho^{\prime}=\max \left[\rho+\delta, \max \left|y_{i}\right|+(n+1)(n+\sqrt{n+1}) \max _{i}\left|y_{i}-w_{i}\right|+\right. \\
\left.(n+\sqrt{n+1})\left|\sum_{j=1}^{n}\left(w_{j}-y_{j}\right)\right|\right] .
\end{array}
$$

Moreover, let $\sigma\left(Y^{1}, \pi(T)\right), T \in Z$, be a t-simplex of the triangulation $G(T)$ of $A(T)$ such that $\max _{i}\left|x_{i}\right|>\rho^{\prime}$ for some $x \in \sigma$. We prove that $\sigma$ has no T-complete facets.

In the following let $\mu_{n+1}=0$ if $n+1 \in T$. By multiplying the system of linear equations with respect to $\sigma$ by $\left((w-x)^{\top}, 0\right)$, we obtain

$$
\begin{aligned}
\sum_{i=1}^{t+1} \lambda_{i}\left(f\left(y^{i}\right)-y^{i}+e\right)^{\top}(w-x) & +\sum_{h \in I_{n} \backslash T} \mu_{h}\left(w_{h}-x_{h}\right)= \\
& =\sum_{j=1}^{n}\left(w_{j}-x_{j}\right) .
\end{aligned}
$$

Moreover, from (7.5.1) we have

$$
(n+1) \sum_{i=1}^{t+1} \lambda_{i}+\sum_{h \notin T} \mu_{h}=n+1 .
$$

Hence,

$$
\begin{aligned}
\sum_{i=1}^{t+1} \lambda_{i}\left(f\left(y^{i}\right)-y^{i}\right)^{\top}(w-x)+ & \sum_{h \in I_{n} \backslash T} \mu_{h}\left(w_{h}-x_{h}\right)- \\
& (n+1)^{-1} \sum_{h \notin T} \mu_{h} \sum_{j=1}^{n}\left(w_{j}-x_{j}\right)=0 .
\end{aligned}
$$

From Merrill's condition it follows that $\left(f\left(y^{i}\right)-y^{i}\right)^{\top}(w-x)$ is positive for all vertices $y^{i}$ of $\sigma$. So, $\sigma$ has no $T$-complete facets if

$$
\text { (7.6.2) } \quad w_{h}-x_{h}-(n+1)^{-1} \sum_{j=1}^{n}\left(w_{j}-x_{j}\right)>0 \quad \text { for } h \in I_{n} \backslash T
$$

and

$$
\text { (7.6.3) } \sum_{j=1}^{n}\left(w_{j}-x_{j}\right)<0 \quad \text { if }(n+1) \notin T .
$$

Observe that at least one $\lambda_{i}$ or $\mu_{h}$ must be positive.

First we prove inequality (7.6.2). Suppose that for some $k_{,} x_{k}=\max _{i}\left|x_{i}\right|$. Then for all $h \in I_{n} \backslash T$ 


$$
\begin{aligned}
x_{k}>\rho^{\prime} \geq y_{k}+(n+\sqrt{n+1}) & \left(y_{h}-w_{h}\right)+n(n+\sqrt{n+1})\left(y_{h}-w_{h}\right)+ \\
& +(n+\sqrt{n+1}) \sum_{j=1}^{n}\left(w_{j}-y_{j}\right) .
\end{aligned}
$$

By the structure of the triangulation matrix we know that

$$
x_{h} \leq y_{h}-(n+\sqrt{n+1})^{-1}\left(x_{k}-y_{k}\right) \quad \text { for } h \in I_{n} \backslash T
$$

Hence, for all $h \in I_{n} \backslash T$

$$
x_{h}<y_{h}-\left(y_{h}-w_{h}\right)-n\left(y_{h}-w_{h}\right)-\sum_{j=1}^{n}\left(w_{j}-y_{j}\right)
$$

and so

$$
\begin{gathered}
x_{h}<w_{h}-(n+1)^{-1} \sum_{j=1}^{n}\left(w_{j}-y_{j}\right)-n(n+1)^{-1}\left(y_{h}-w_{h}\right) \\
-n(n+1)^{-1}\left(w_{h}-x_{h}\right) .
\end{gathered}
$$

Since for $h \in I_{n} \backslash T, y_{h}-x_{h} \geq y_{j}-x_{j}$ for $j \in I_{n}$, we obtain

$$
\begin{aligned}
x_{h} & <w_{h}-(n+1)^{-1} \sum_{j=1}^{n}\left(w_{j}-y_{j}\right)-(n+1)^{-1} \sum_{j=1}^{n}\left(y_{j}-x_{j}\right) \\
& <w_{h}-(n+1)^{-1} \sum_{j=1}^{n}\left(w_{j}-x_{j}\right)
\end{aligned}
$$

which proves (7.6.2) if $x_{k}=\max \left|x_{i}\right|$ for some $\mathrm{k}$.

On the other hand, if $-x_{k}=\max \left|x_{i}\right|$ for some $k$, then we have again from the structure of the triangulation matrix that

$$
x_{h}=y_{h}+\left(x_{h}-y_{h}\right) \leq y_{h}+\left(x_{k}-y_{k}\right) \text { for } h \in I_{n} \backslash T \text {. }
$$

Together with

$$
-x_{k}>\rho^{\prime} \geq-y_{k}+(n+1)\left(y_{h}-w_{h}\right)+\sum_{j=1}^{n}\left(w_{j}-y_{j}\right)
$$

this implies

$$
x_{h}<y_{h}-(n+1)\left(y_{h}-w_{h}\right)-\Sigma_{j}\left(w_{j}-y_{j}\right) .
$$


Hence, for $h \in I_{n} \backslash T$

$$
n(n+1)^{-1}\left(y_{h}-x_{h}\right)+\left(x_{h}-w_{h}\right)+(n+1)^{-1} \Sigma_{j}\left(w_{j}-y_{j}\right)<0 .
$$

Again since $y_{h}-x_{h} \geq y_{j}-x_{j}$ for all $j \in I_{n}$ '

$$
(n+1)^{-1} \Sigma_{j}\left(y_{j}-x_{j}\right)+\left(x_{h}-w_{h}\right)+(n+1)^{-1} \Sigma_{j}\left(w_{j}-y_{j}\right)<0
$$

and so

$$
w_{h}-x_{h}-(n+1)^{-1} \sum_{j=1}^{n}\left(w_{j}-x_{j}\right)>0,
$$

which completes the first part of the proof. It remains to prove that $\Sigma_{j}\left(w_{j}-x_{j}\right)<0$ if $n+1 \notin T$.

Suppose again that $x_{k}=\max _{i}\left|x_{i}\right|$ for some $k$. Since $n+1 \notin T$, we must have that

$$
\sum_{i \neq k} x_{i} \geq \sum_{i \neq k} y_{i}-(n-1)(n+\sqrt{n+1})^{-1}\left(x_{k}-y_{k}\right)
$$

Hence,

$$
\Sigma_{j}\left(w_{j}-x_{j}\right) \leq \Sigma_{j}\left(w_{j}-y_{j}\right)-(1+\sqrt{n+1})(n+\sqrt{n+1})^{-1}\left(x_{k}-y_{k}\right) .
$$

The right side of this inequality is negative since

$$
x_{k}>\rho^{\prime} \geq y_{k}+(n+\sqrt{n+1})(1+\sqrt{n+1})^{-1} \sum_{j}\left(w_{j}-y_{j}\right) .
$$

Finally, let $-y_{k}=\max \left|y_{i}\right|$ for some $k$. Then

$$
-x_{k}>\rho^{\prime} \geq-y_{k}+(1+\sqrt{n+1})^{-1} \Sigma_{j}\left(w_{j}-y_{j}\right) .
$$

Since $(n+1) \notin T$ and $y_{k}-x_{k} \geq 0$ we have, again by the structure of the triangulation matrix, that

$$
\sum_{i \neq k}\left(x_{i}-y_{i}\right) \geq(2+\sqrt{n+1})\left(y_{k}-x_{k}\right)
$$


Therefore,

$$
\Sigma_{j}\left(w_{j}-x_{j}\right)=\Sigma_{j}\left(w_{j}-y_{j}\right)+\sum_{i \neq k}\left(y_{i}-x_{i}\right)+\left(y_{k}-x_{k}\right)<0,
$$

which completes the proof of the theorem.

The theorem can easily be generalized for any $(\alpha, \beta)$-triangulation as long as $\beta$ is negative. Note that the only difference with the proof for the standard labelling rule is that in that case

$$
\text { (n+1) } \sum_{i=1}^{t+1} \lambda_{i}+\mu_{n+1}=n+1,
$$

whereas in the case of the alternative labelling rule

$$
(n+1) \sum_{i=1}^{t+1} \lambda_{i}+\sum_{h \notin T} \mu_{h}=n+1 .
$$

As mentioned in the introduction, Reisex [1978a, 1978b] found independently an algorithm for the NLCP. This algorithm is closely related to the extreme case $\mathrm{N}=\mathrm{n}$. However, Reiser's algorithm is based on the $\mathrm{K}$ triangulation and was only presented for integer labelling. Reiser uses the same (integer) labelling rule as we have. If in his algorithm label $(j, 1)$ is a new label for some $j \in I_{n^{\prime}}$ then the vertex $y^{t+1}=y^{t}+D e(1)$ is added to the current simplex $\sigma\left(y^{1}, \ldots, y^{t}\right)$ just as in our algorithm. However, if label $(j, 2)$ is just found, $\sigma\left(y^{1}, \ldots, y^{t}\right)$ is extended by adding the vertex $y^{1}-D e(j)$ instead of the vertex $y^{t}-D e(j)$. Clearly, Reiser's algorithm generates simplices of the $\mathrm{K}$ triangulation whereas our algorithm generates simplices of the K'triangulation. As argued before the latter triangulation takes advantage of knowing the starting point. Therefore, we may expect that our method takes on the average fewer iterations than Reiser's algorithm (see also section 7).

Comparing our algorithm in the case $\mathrm{N}=\mathrm{n}$ with the basic variable dimension algorithm discussed in chapter 4, we can say that for integer labelling the accuracy of the latter is much better (on the average). We now explain the reason of this phenomenon. If a completely labelled simplex is found, 
say $\sigma\left(y^{1}, \ldots, y^{n+1}\right)$, we have the property that for all indices there is at least one vertex $y^{i_{1}}$, such that $f_{i}\left(y^{i_{1}}\right)>y_{i}{ }^{i}$ and also at least one vertex $y^{i_{2}}$ such that $f_{i}\left(y^{i_{2}}\right)<y_{i}^{i_{2}}$. Hence all components of $f(x)-x, x \in \sigma$, are very close to zero. However, for a j-stopping face we only know that there is at least one vertex $y^{j_{1}}$ with $f_{j}\left(y^{j_{1}}\right)>y_{j}^{j_{1}}$ and one vertex $y^{j_{2}}$ with $f_{j}\left(y^{j 2}\right)<y_{j}^{j}$. Hence $f_{j}(x)-x_{j}$ is very close to zero for $x \in \sigma$.

Concerning the other components of $f(x)-x$, they must also be close to zero because of the labelling rule but they can be positive (or negative) for all $\mathrm{x}$ in $\sigma$. This affects the accuracy of $\mathrm{x}$ as approximate fixed point. Therefore we propose the following labelling rule in case $\mathrm{N}=\mathrm{n}$.

DEFINITION 7.6.2. Let $\mathrm{y}$ be the starting point and for $\mathrm{x} \in \mathrm{R}^{\mathrm{n}}$ let $J(\mathrm{x})$ be the set of indices

$$
J(x)=\left\{(j, 1) \mid x_{j} \geq y_{j}\right\} \cup\left\{(j, 2) \mid x_{j} \leq y_{j}\right\} .
$$

Furthermore, let $J^{*}(x)$ be the union of all those $(j, 1) \in J(x)$ for which

$$
f_{j}(x)-x_{j}=h(x)
$$

and all those $(j, 2) \in J(x)$ for which

$$
f_{j}(x)-x_{j}=-h(x)
$$

where $h(x)=\left|\max \left\{\max _{(i, 1) \in J(x)}\left(f_{i}(x)-x_{i}\right), \max _{(i, 2) \in J(x)}\left(-f_{i}(x)+x_{i}\right)\right\}\right|$.

Then the point $\mathrm{x}$ receives the label $\ell(\mathrm{x})$ where

$$
\begin{array}{ll}
\ell(x)=\min \left\{(j, h) \mid(j, h) \in J^{*}(x)\right\} & \text { if } J^{*}(x) \neq \varnothing \\
\ell(x)=0 & \text { if } J^{*}(x)=\varnothing .
\end{array}
$$

Note that $x$ can only receive label 0 if $x_{i} \neq y_{i}$ for all $i$, i.e. if the algorithm operates with full-dimenional simplices. Recall that $x_{i}=y_{i}$ if $x \in \AA(T)$ with both $(i, 1)$ and $(i, 2) \notin T$. The set $J(x)$ of definition 7.6.2 is the union of $T$ where $x \in \AA(T)$ and the set of indices $(j, 1)$ and $(j, 2)$ 
which are both not in $T$, i.e. if $x \in \stackrel{\circ}{\AA}(T)$ then $J(x)$ is the set of indices $(i, h)$ such that $\left(i, h^{\prime}\right), h^{\prime} \neq h$, is not an element of $T$. Within this set of indices, the labelling rule takes the "worst" component of $f(x)-x$ as label. Hence, the labelling rule postpones to find a $j$-stopping face as long as possible. If $J^{*}(x)$ is empty, i.e. if label 0 is found, then we have the property that for all $i$ there is both a vertex $y^{i 1}$ such that $f_{i}\left(y^{i_{1}}\right)>y_{i}^{i_{1}}$ and a vertex $y^{i_{2}}$ such that $f_{i}\left(y^{i_{2}}\right)<y_{i}^{i_{2}}$ (if $\sigma\left(y^{1}, \ldots, y^{n+1}\right.$ ) is the current simplex). Hence, if the algorithm terminates with a simplex having label 0 in the label set, the accuracy is just the same as in the basic algorithm. But it is still possible that the algorithm terminates with a (lower-dimensional) j-stopping simplex. This occurs if the new vertex whose label is equal to $(j, 2$ ) (say) is a proper point of the region $A\left(T_{1}\right)$ while $T_{1}$ is a proper subset of the current label set $T$ such that $(j, 1)$ is an element of $T \backslash T_{1}$. The latter is illustrated in figure 7.6.1 for $n=3$. In this figure the point $a=y+3 d_{1} e(1)$ has label $(2,2)$ whereas $(1,1)$ and $(2,1)$ form the current label set. However, $a \in A(\{(1,1)\})$ so that only label $(1,2)$ is excluded for determining $l(a)$.

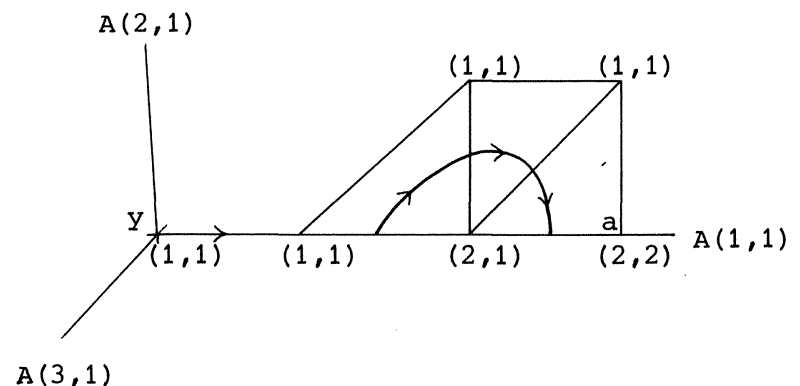

Figure 7.6.1. Alternative labelling rule.

Even when the algorithm terminates with a lower-dimensional simplex, one may expect that the accuracy is much better then for the previous integer labelling rule since for any point $x \neq y$ the assignment of a label depends on the region in which $\mathrm{x}$ is a proper point. Note that the labelling rule heavily depends on the starting point. The original integer labelling rule, however, has the advantage that sometimes a connected set of fixed points can be approximated by continuing the algorithm according to the steps 1-4 
discussed in section 6.4. By way of illustration we give a two-dimensional example.

EXAMPLE 7.6.3. Let the continuous function $f: R^{2} \rightarrow R^{2}$ be defined by

$$
f_{1}(x)=\frac{1}{2} x_{1}+\frac{1}{4} \quad x_{1} \in R
$$

and

$$
f_{2}(x)=\left\{\begin{array}{lc}
\frac{1}{4} & x_{2} \leq \frac{1}{4} \\
x_{2} & \frac{1}{4}<x_{2} \leq \frac{3}{4} \\
\frac{3}{4} & x_{2}>\frac{3}{4}
\end{array}\right.
$$

For the two extreme cases $N=1$ and $N=2$ the regions of the points having the same label are shown in the figures $7.6 .2 \mathrm{a}$ respectively $7.6 .2 \mathrm{~b}$.

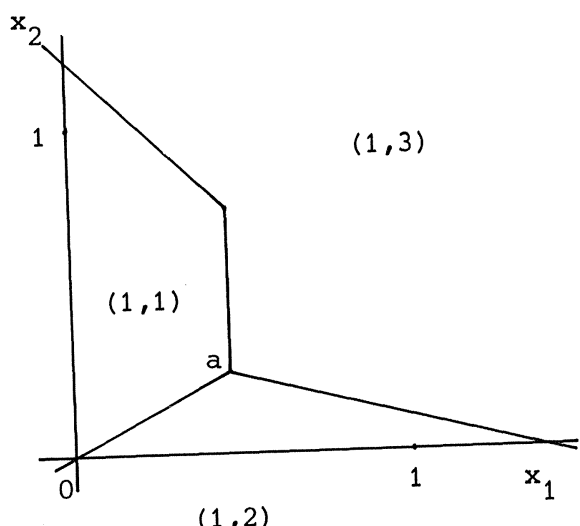

$(1,2)$

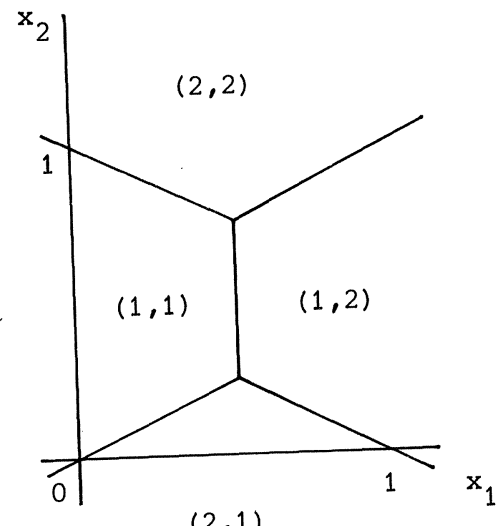

$(2,1)$

Figure 7.6.2a. Labelling if $\mathrm{N}=1$. Figure 7.6.2b. Labelling if $\mathrm{N}=2$.

Observe that the set $\left\{\left(x_{1}, x_{2}\right) \mid x_{1}=\frac{1}{2}, \frac{3}{4} \leq x_{2} \leq \frac{3}{4}\right\}$ is the set of fixed points of $\mathrm{f}$. For $\mathrm{N}=1$ there exists for every grid size only one completely labellied simplex, viz. the simplex containing the point a. Hence, independent of the starting point, a sequence of completely labelled simplices converges to the point $\left(\frac{1}{2}, \frac{1}{4}\right)$ if the mesh goes to zero. So, other fixed points cannot be approximated. However, if $\mathrm{N}=2$, different starting points may give different (approximate) fixed points as is illustrated in figure 7.6.3 for $d_{1}=d_{2}=1 / 3$. In this figure the simplex $\tau$ is the 
stopping simplex of the algorithm. As the mesh goes to zero, the points $\left(\frac{1}{2}, \frac{1}{4}\right),\left(\frac{1}{2}, \frac{1}{2}\right)$ and $\left(\frac{1}{2}, \frac{3}{4}\right)$ respectively are found as fixed points.

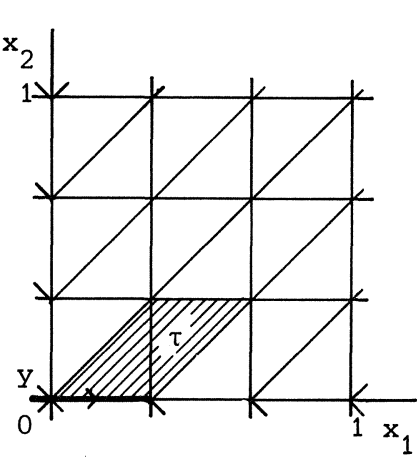

a. $y=(0,0)$.

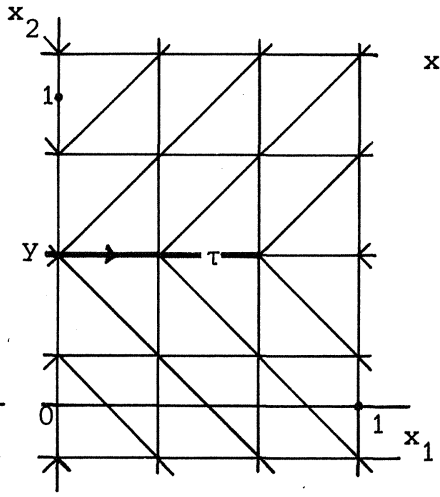

b. $y=\left(0, \frac{1}{2}\right)$.

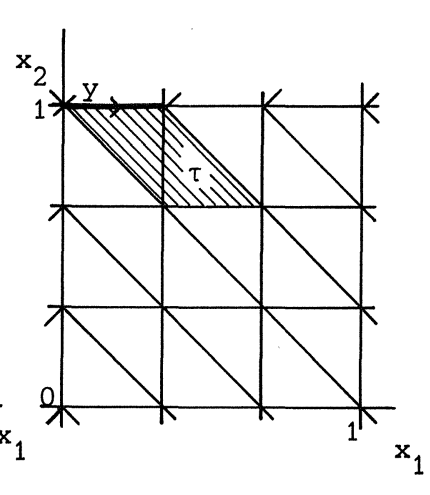

c. $y=(0,1)$

Figure 7.6.3.

To approximate a set of fixed points if for some $j$ a $t$-dimensional $j$-stopping simplex $\sigma\left(w^{1}, \pi(T)\right)$ of $G(T)$ is found, suppose first that $t>1$. Then there exists an index s such that $l\left(w^{\mathbf{S}}\right) \neq(j, h)$ for both $h=1,2$, i.e. $\ell\left(w^{5}\right)=\left(i^{*}, h^{*}\right)$ for some $i^{*} \neq j$ and $h^{*} \epsilon\{1,2\}$. Clearly the facet $\tau$ opposite $w^{\mathbf{S}}$ is still a good approximation of a fixed point since both $(j, 1)$ and $(j, 2)$ are elements of its label set. So, we can continue the algorithm by removing the vertex $w^{\mathbf{s}}$ of $\sigma$, etc. Hence, a path of adjacent j-stopping simplices is generated. If, however, in step 4 the index $\left(i^{*}, h^{*}\right)$ should be deleted (note that $\left(i^{*}, h^{*}\right)$ is an element of $T$ but not of the label set), then instead of deleting $y^{t}$ this vertex becomes $y^{t}=y^{t-1}-D q\left(i^{*}, h^{*}\right)$. Also, $T$ becomes equal to $T \cup\left\{\left(i^{*}, h\right)\right\} /\left\{\left(i^{*}, h^{*}\right)\right\}$ where $h \neq h^{*}$. The procedure terminates as soon as label $\left(i^{*}, 1\right)$ or label $\left(i^{*}, 2\right)$ is found or if for some $i \neq j$ the current simplex appears to be also an $i$-stopping simplex. Since all steps are unique the just described algorithm can never return in a previously visited simplex except the one with which the continuation was started, i.e. the simplex $\sigma\left(w^{1}, \ldots, w^{s}, \ldots, w^{t}\right)$. However, since $l\left(w^{s}\right)=\left(i^{*}, h^{*}\right)$ the method-terminates if $\sigma$ is revisited. Of course, the same procedure can be performed for all vertices $w^{i}$ of $\sigma$ having a label unequal to $(j, 1)$ or $(j, 2)$. Moreover, the algorithm can be continued in other $j$-(or $i-$ ) stopping simplices. The procedure is illustrated for the example with $y=(0,0)$ in 
in figure 7.6 .4 if the vertex $(1 / 3,0)$ of $\tau$ is removed. Then the method terminates with the simplex $\sigma$ whose vertex $(2 / 3,1)$ has label $(2,2)$. Note that doing so a path is followed which connects the regions having label $(2,2)$ and label $(2,1)$. The path is such that it follows the intersection of the (closure of) the regions having label $(1,1)$ and $(1,2)$. A similar result will be obtained for the third starting point.

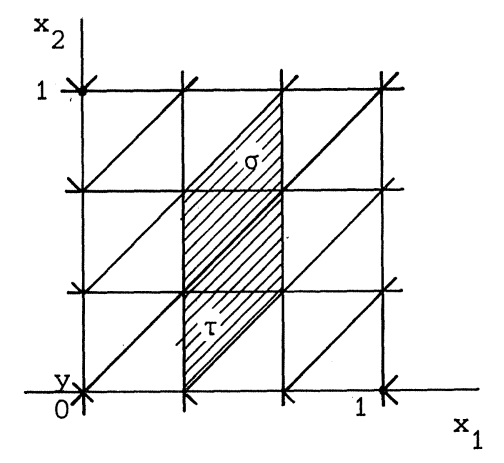

Figure 7.6.4. Approximation of a set of fixed points if $t>1$.

If $t$ is equal to one, there is no vertex $w^{s}$ such that $\ell\left(w^{s}\right) \neq(j, h)$, $h=1,2$. But if $t \leq n-1$, there is at least one index $i^{*} \neq j$ such that for all vertices $w^{s} l\left(w^{s}\right) \neq\left(i^{*}, h\right), h=1,2$. Hence, the current simplex can be extended to a $(t+1)$-simplex of the triangulation $G\left(T \cup\left\{\left(i^{*}, h^{*}\right)\right\}\right)$ by adding the vertex $w^{t+1}=w^{t}+D q\left(i^{*}, h^{*}\right)$ for some $h^{*} \in\{1,2\}$. This simplex is still a j-stopping simplex. By computing $l\left(w^{t+1}\right)$ we continue in exactly the same way as described above for the case $t>1$. As soon as label $\left(i^{*}, 1\right)$ or label $\left(i^{*}, 2\right)$ is found (or an $i$-stopping simplex, $i \neq j$ ) the procedure terminates. For the example this is illustrated in figure 7.6.5 if $\mathrm{y}=\left(0, \frac{1}{2}\right)$. Starting with $\left(i^{*}, \mathrm{~h}^{*}\right)=(2,1)$ the algorithm terminates with $\sigma_{1}$ since label $(2,2)$ is then found, and starting with $\left(i^{*}, h^{*}\right)=(2,2)$ it terminates with $\sigma_{2}$. In the latter case label $(2,1)$ is found. The union of both paths of simplices yields a set of approximate fixed points of $f$. of course, if the grid sizes become smaller, the approximation will become better whereas the total number of iterations increases. 

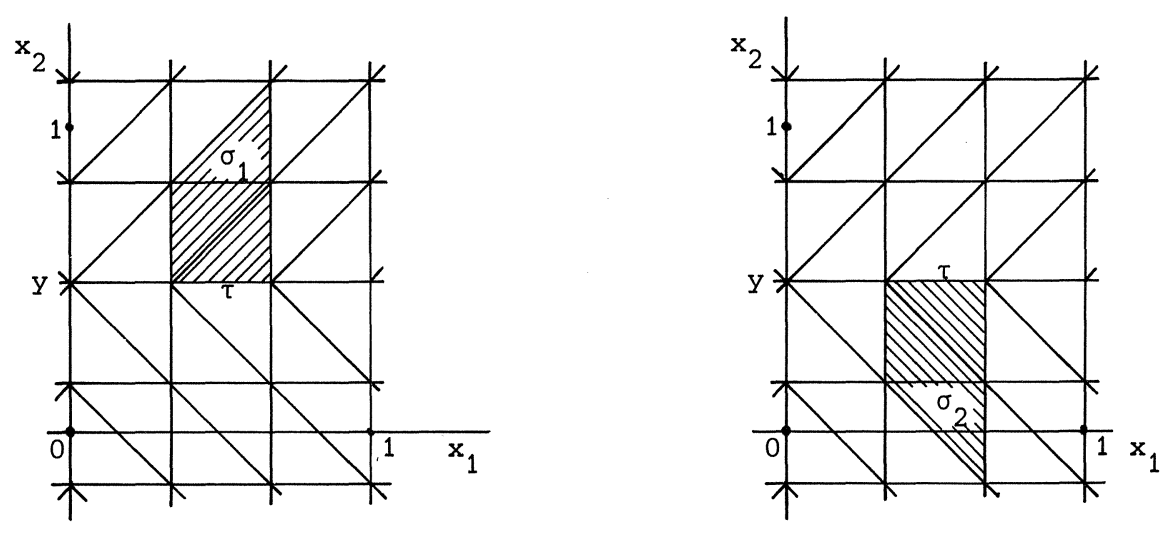

Figure 7.6.5. Approximation of a set of fixed points if $t<n-1$.

Concerning vector labelling we have no idea how to continue the algorithm in a completely labelled simplex to approximate a set of fixed points. Observe that the system of $\mathrm{N}+\mathrm{n}$ linear equations becomes completely degenerated as soon as for some $j$ a $j$-stopping simplex is found.

In the next theorem we prove that Merrill's condition is sufficient to guarantee convergence if vector labelling is used (see also van der Laan and Talman [1980e] and Todd [1980]).

THEOREM 7.6.4. In case $\mathrm{N}=\mathrm{n}$ the algorithm converges under Merrill's condition if the mesh of the triangulation is small enough.

PROOF. Let mesh $G<\delta$ and let $y$ be the starting point. Define $\rho$ ' by

$$
\rho^{\prime}=\max \left\{\rho+\delta, \max _{i \in I_{n}}\left|y_{i}\right|+\sum_{j=1}^{n}\left|w_{j}-y_{j}\right|\right\} \text {. }
$$

Furthermore, let for some $T \in Z, \sigma\left(y^{1}, \pi(T)\right)$ be a simplex of $G(T)$ such that $\max _{i}\left|x_{i}\right|>\rho^{\prime}$ for some $x \in \sigma$. We will prove that $\sigma$ has no $T$-complete facets. After multiplying the system of linear equations oorresponding to $\sigma$ with $\left((w-x)^{\top},(x-w)^{\top}\right)$ we obtain 


$$
\begin{array}{r}
\sum_{i=1}^{t+1} \lambda_{i}\left(f\left(y^{i}\right)-y^{i}+e\right)^{\top}(w-x)+\sum_{i=1}^{t+1} \lambda_{i}\left(-f\left(y^{i}\right)+y^{i}+e\right)^{\top}(x-w) \\
+\sum_{j=1}^{n} \mu_{j, 1}(w-x)_{j}+\sum_{j=1}^{n} \mu_{j, 2}(x-w)_{j}=0
\end{array}
$$

and so

$$
\text { (7.6.4) } \quad 2 \sum_{i=1}^{t+1} \lambda_{i}\left(f\left(y^{i}\right)-y^{i}\right)^{\top}(w-x)+\sum_{j=1}^{n}\left(\mu_{j, 1}-\mu_{j, 2}\right)(w-x)_{j}=0 \text {, }
$$

where $\mu_{j, k}=0$ if $(j, k) \in T$. By Merrill's condition $\left(f\left(y^{i}\right)-y^{i}\right)^{\top}(w-x)>0$ for all vertices $y^{i}$ of $\sigma$. So, to prove that the system has no feasible solution, it is sufficient to show that the second term on the left side is positive. Clearly, $\Sigma_{i} \lambda_{i}<1$ since otherwise $\mu_{j, 1}$ and $\mu_{j, 2}$ are equal to zero for all $j$ which contradicts $(7.6 .4)$. If $\mu_{j, 1}>0$, then $(j, 1) \notin T$ and so $x_{j} \leq y_{j}$. In the same way, $\mu_{j, 2}>0$ implies $x_{j} \geq y_{j}$. Hence, $\mu_{j, 1}\left(y_{j}-x_{j}\right) \geq 0$ and $\mu_{j, 2}\left(x_{j}-y_{j}\right) \geq 0$ for all $j$. Observe from equation (7.5.1) that $\mu_{j, 1}+\mu_{j, 2}=2-2 \Sigma_{i} \lambda_{i}>0$. Suppose now that $\mathrm{x}_{\mathrm{s}}=\max _{\mathrm{i}}\left|\mathrm{x}_{\mathrm{i}}\right|$ for some $\mathrm{s}$. Clearly, $(s, 1) \in \mathrm{T},(s, 2) \notin \mathrm{T}$, $\mu_{s, 2}=2-2 \Sigma_{i} \lambda_{i}>0$ and

$$
x_{s}>\rho^{\prime}>y_{s}+\sum_{j=1}^{n}\left|w_{j}-y_{j}\right| \text {. }
$$

Therefore,

$$
\begin{aligned}
& \sum_{j=1}^{n}\left(\mu_{j, 1}-\mu_{j, 2}\right)\left(w_{j}-x_{j}\right) \\
& \geq \sum_{j=1}^{n}\left\{\mu_{j, 1}\left(w_{j}-y_{j}\right)+\mu_{j, 2}\left(y_{j}-w_{j}\right)\right\}+\mu_{s, 2}\left(x_{s}-y_{s}\right) \\
& >\mu_{s, 2}\left\{\mu_{s, 2}^{-1} \sum_{j=1}^{n} \mu_{j, 1}\left(w_{j}-y_{j}\right)+\mu_{s, 2}^{-1} \sum_{j=1}^{n} \mu_{j, 2}\left(y_{j}-w_{j}\right)+\sum_{j=1}^{n}\left|w_{j}-y_{j}\right|\right\} \\
& \geq \mu_{s, 2}\left\{-\mu_{s, 2}^{-1} \sum_{j=1}^{n}\left(\mu_{j, 1}+\mu_{j, 2}\right)\left|w_{j}-y_{j}\right|+\sum_{j=1}\left|w_{j}-y_{j}\right|\right\} \\
& =0,
\end{aligned}
$$


where in the last inequality we use $\mu_{j, 1}+\mu_{j, 2}=\mu_{s, 2}$ for all $j$, as follows from equation (7.5.1). Consequently, $\sigma$ has no T-complete facets. On the other hand if $x_{s}=-\max _{i}\left|x_{i}\right|$ for some $s$, then $(s, 2) \in T,(s, 1) \notin T$, $\mu_{s, 1}=2-2 \Sigma_{i} \lambda_{i}>0$ and

$$
-x_{s}>\rho^{\prime} \geq-y_{s}+\sum_{j=1}^{n}\left|w_{j}-y_{j}\right| \text {. }
$$

Following the same arguments as above we again obtain that

$$
\sum_{j=1}^{n}\left(\mu_{j, 1}-\mu_{j, 2}\right)\left(w_{j}-x_{j}\right)>0
$$

Combining all of this together we have that no simplices outside $B\left(\rho^{\prime}\right)$ can be generated. Hence, the algorithm terminates within a finite number of iterations.

Now we give an application due to Todd and Wright [1979]. Let $B^{n}=\left\{x \in R^{n}\left|\max _{i}\right| x_{i} \mid \leq 1\right\}$.

THEOREM 7.6.5. If $\mathrm{f}: \mathrm{B}^{\mathrm{n}} \rightarrow \mathrm{R}^{\mathrm{n}}$ is continuous, and if $\mathrm{f}(-\mathrm{y})=-\mathrm{f}(\mathrm{y})$ for all $y \in$ bd $B^{n}$, then there exists an $x^{*} \in B^{n}$ such that $f\left(x^{*}\right)=0$.

This theorem is a version of the Borsuk-Ulam theorem. To apply the algorithm for proving the theorem and to compute a źero-point of $f$, we have to adapt the triangulation and the labelling rule. First we take a triangulation $\mathrm{G}$ of $\mathrm{B}^{\mathrm{n}}$ which is centrally symmetric and subdivides the orthants of $\mathrm{B}^{\mathrm{n}}$, e.g. the $\mathrm{K}^{\prime}$ triangulation with $\mathrm{y}=\underline{0}$ and $a_{j}=m^{-1}$ for all $j$. So, if $\tau$ is a boundary-face of $G$, then $-\tau$ is also a boundary-face of $G$. Moreover, let for $T \in Z$ the region $A(T)$ be defined as in section 2 for $Y=0$, i.e. $A(T)$ is the suborthant

$$
A(T)=\left\{x \in B^{n} \mid x=\underset{(j, k) \in T}{\sum} \lambda_{j, k} q(j, k) \text { for nonnegative } \lambda_{j, k}\right\}
$$

where $q(j, 1)=e(j)$ and $q(j, 2)=-e(j)$. Then each $A(T)$ is triangulated by $G$ restricted to $A(T)$. Let $G(T)$ be this triangulation of $A(T)$. Concerning the labelling rule, each point $x \in B^{n}$ receives a label according to the standard labelling rule with respect to $f(x)$ (instead of $f(x)-x)$. For $\mathrm{T} \in \mathrm{Z}, \mathrm{T}$-completeness is defined as in the sections 3 and 5 . Then we have the following important lemma. 
LEMMA 7.6.6. For $T \in Z$ let $\tau$ be a $T$-complete facet of $G(T)$ such that $\tau$ lies in the boundary of $B^{n}$. Then the facet- $\tau$ is a $T^{\prime}$-complete facet of the triangulation $G\left(T^{\prime}\right)$ in bd $B^{n}$, where $T^{\prime} \in Z$ is such that

$$
\left(j, h_{1}\right) \in T^{\prime} \quad \text { if and only if }\left(j, h_{2}\right) \in T, \quad h_{1} \neq h_{2} .
$$

The algorithm now starts in the point $\underline{0}$ and operates as described in the sections 4 and 5 with some modifications. If in step 1 a T-complete facet $\tau$ in the boundary of $B^{n}$ is generated, then the algorithm continues on the other side of $B^{n}$ by putting $\tau=-\tau$ and $T=T^{\prime}$ whereas $\sigma$ and $R$ are adapted such that $\sigma$ is the (unique) t-simplex in $G(T)$ having the new $\tau$ as a facet and such that $R$ gives the number of steps between the starting point $\underline{0}$ and the vertex $\mathrm{y}^{1}$ of $\sigma$. If the $\mathrm{K}^{\prime}$ triangulation underlies the algorithm, then $\sigma$ becomes $-\sigma$ whereas the $(j, 1)$ and $(j, 2)$ components of $R$ are interchanged for all j. Clearly, this switch step is unique and feasible and does not affect the convergence-proof. We refer to Todd and Wright [1979] to solve the problem how to start with another point than $\underline{0}$. They also propose in their paper a practical way of handling degeneracy. It is based on a lexicographic pivoting rule. Moreover, they give in the same paper an application of the Borsuk-Ulam theorem to a system of polynomial equations. Finally we make some remarks. Wolsey [1973], Saigal [1977c], Saigal and Todd [1978] have studied the rate of convergence that can be achieved by simplicial algorithms operating in $\mathrm{R}^{\mathrm{n}}$ with vector labelling. To obtain fast convergence when the function is smooth, Saigal and Todd [1978] take an approximate Newton-step with the approximate fixed point $\mathrm{x}^{\mathrm{k}}$ before the $k$-th restart is made. Therefore they make use of the approximate Jacobian in the point $x^{k}$. This matrix can easily be derived from the system of linear equations corresponding to the completely labelled simplex containing $\mathrm{x}^{\mathrm{k}}$. The mesh of the triangulation will then be taken as the minimum of the length of the Newton-step and one half of the previous mesh. Furthermore, the result of the Newton-step becomes the new starting point $\mathrm{y}^{\mathrm{k}}$ rather than $\mathrm{x}^{\mathrm{k}}$. Under some rather strong conditions of the function $f$, the algorithm will find in the limit in exactly $n+1$ pivot steps a completely labelled simplex. Todd [1878b,1980] treated this matter for Van der Laan and Talman's basic algorithm and for the algorithm described in this chapter for the case $N=n$. Recently, Saigal discovered how the rate of convergence depends on the underlying 
triangulation (see saigal [1979]). The $(n+\sqrt{n+1})$-triangulation in case $\mathrm{N}=1$ and the $\mathrm{K}^{\prime}$ triangulation in case $\mathrm{N}=\mathrm{n}$ seem to be the triangulations involving the highest convergence rate for the variable dimension algorithm on $\mathrm{R}^{\mathrm{n}}$. Wright [1979] recently introduced a variable dimension algorithm on $\mathrm{R}^{\mathrm{n}}$ with $2^{\mathrm{n}}$ directions, which can be considered to be dual to the $2 \mathrm{n}$-case. His algorithm was based on the algorithm described in this chapter for the case $N=n$. Finally, we remark that Todd and wright [1979] found a geometric interpretation for our algorithm for the case $N=n$. In chapter 8 we generalize this interpretation for the whole class of algorithms. Moreover, we give in the same chapter two other geometric interpretations with only $\mathrm{n}+\mathrm{N}$ artificial points on an extra level (instead of $\left.\Pi_{j=1}^{N}\left(m_{j}+1\right)\right)$.

\subsection{COMPUTATIONAL EXPERIENCE .}

For both extreme cases the algorithm was applied for vector labelling to the same ten-dimensional variant of the problem considered by Kellog, $\mathrm{Li}$ and Yorke [1976] as in chapter 5. First, ten starting points $\mathrm{y}$ were at random chosen such that $0<y_{i}<\frac{1}{2}$ for all $i$. For $N=1$ the algorithm was applied for both the $\mathrm{K}$ and $(\mathrm{n}+\sqrt{\mathrm{n}+1})$-triangulation. For all applications the mesh was equal to $\sqrt{10} / 40$. Only one run was made so that we obtain a good comparison of the number of iterations if large movements have to be made. The accuracy was about $10^{-5}$ for $\mathrm{N}=1$ and $10^{-4}$ for $\mathrm{N}=10$. The results are given in table 7.7.1.

Clearly, for all runs the computation time of one function-evaluation is the same (on the average). However, if $\mathrm{N}=1$ we have to perform a linear programming step in an $(n+1)$-dimensional matrix during each iteration, whereas if $\mathrm{N}=\mathrm{n}$ a pivot step has to be made in a $2 \mathrm{n}$-dimensional matrix. Therefore we may expect that the computation time of a pivot step is about 4 times as much for $\mathrm{N}=\mathrm{n}$ as for $\mathrm{N}=1$. Observe, however, that the algorithm starts always with an identity matrix. If $\mathrm{N}=1$, the method terminates as soon as $n+1$ unit-columns have been eliminated. The same holds for $N=n$ (or if $1<N<n$ ). Since it is not necessary to store or update unit columns, the difference in computation time to perform a pivot step is quite modest. The difference disappears at all if a parallel machine is used (see Forster [1980]). For integer labelling the algorithm for $\mathrm{N}=\mathrm{n}$ was applied to the 20 - and 80 - dimensional problems 


\begin{tabular}{c|cc|c} 
& \multicolumn{2}{|c|}{$N=1$} & $N=\mathrm{N}$ \\
\hline Starts & $\mathrm{K}$ & $\mathrm{A}^{*} \mathrm{~K}$ & \multicolumn{1}{c}{$\mathrm{K}^{\prime}$} \\
\hline 1 & 153 & 109 & 117 \\
2 & 153 & 91 & 67 \\
3 & 153 & 88 & 101 \\
4 & 182 & 112 & 97 \\
5 & 168 & 97 & 76 \\
6 & 205 & 129 & 74 \\
7 & 191 & 101 & 96 \\
8 & 104 & 55 & 72 \\
9 & 185 & 117 & 85 \\
10 & 200 & 131 & 121 \\
& & &
\end{tabular}

Table 7.7.1. Number of iterations, $\mathrm{n}=10$.

considered by Kellog, Li and Yorke [1976]. For both runs the starting point was put equal to $(1, \ldots, 1)^{\top}$. Moreover, we take $d_{j}=2$ for all $j$ and a factor of incrementation of 2. The results are shown in table 7.7.2.

\begin{tabular}{c|cc|cc} 
& \multicolumn{2}{|c|}{$\mathrm{n}=20$} & \multicolumn{2}{|c}{$\mathrm{n}=80$} \\
\hline Grid size & \multicolumn{1}{|c|}{ iterations } & accuracy & iterations & accuracy \\
\hline .5 & 2 & .8 & 5 & .9 \\
2 & 35 & .2 & 135 & .24 \\
8 & 58 & .05 & 204 & .06 \\
32 & 81 & .01 & 281 & .015 \\
128 & 98 & .003 & 364 & .004 \\
512 & 121 & .008 & 439 & .001
\end{tabular}

Table 7.7.2. Number of iterations, $\mathrm{n}=20$ and $\mathrm{n}=80$.

Observe that the mean number of iterations in each stage is about $\frac{1}{2} \mathrm{n}$ whereas for $N=1$ at least $n+1$ iterations have to be done. For both the alternative labelling rule and the (standard) vector labelling rule we also cannot expect this saving. But then the accuracy is better so that we can increase the factor of incrementation by using the acceleration technique proposed by Saigal and Todd [1978]. Further research has to be 
done to decide which factor of incrementation is "optimal", in particular in case of integer labelling.

Finally, we compare our algorithm for $\mathrm{N}=1$ and $\mathrm{N}=\mathrm{n}$ with Reiser's algorithm. Therefore, we applied both algorithms to a 20-dimensional problem of Garcia and Gould [1978]. Reiser [1978a,page 70] started with a grid size of $3^{-1}$ in the zero-point and used a factor of incrementation of 5. The results are given in table 7.7.3. Since for all runs the number of iterations was the same in the first stage (639), we subtracted this number everywhere. For the case $\mathrm{N}=\mathrm{n}$ the algorithm was applied both for the "standard" labelling rule of section 3 , denoted by $\mathrm{K}_{\mathrm{s}}^{\prime}$, and for the "alternative" labelling of section 6 , denoted by $\mathrm{K}_{\mathrm{a}}^{\prime}$.

\begin{tabular}{c|rrrr} 
Stage & Reiser & \multicolumn{1}{c}{$K$} & $K_{S}^{\prime}$ & $K_{a}^{\prime}$ \\
\hline 2 & 92 & 57 & 41 & 53 \\
3 & 229 & 78 & 80 & 74 \\
4 & 366 & 127 & 157 & 101 \\
5 & 483 & 148 & 190 & 131 \\
6 & 580 & 188 & 258 & 152 \\
7 & 677 & 209 & 305 & 186 \\
8 & 804 & 237 & 369 & 207
\end{tabular}

Table 7.7.3. Number of iterations, $n=20$ :

Observe that the run for $\mathrm{N}=\mathrm{n}$ with the standard labelling rule is only better in the second stage. However, the approximation is so bad that it takes a lot of iterations to find an approximate fixed point in the next stage. This phenomenon is larger as the factor of incrementation increases. The same feature holds for Reiser's algorithm. The accuracy for the latter was comparable with that of our variable dimension algorithm in case $N=n$ with "standard" labelling whereas the other two runs ( $K$ and $K_{a}^{\prime}$ ) were also involved with the same accuracy. 
GEOMETRIC INTERPRETATIONS OF THE ALGORITHMS

\subsection{INTRODUCTION.}

In this chapter three interpretations with an artificial level of points are given for the algorithms presented in the previous chapters. The first interpretation gives a subdivision of the convex hull of the artificial level and the natural level, the second interpretation yields a triangulation and the last one neither a triangulation nor a subdivision. However, for the extreme case $\mathrm{N}=1$ all interpretations coincide and give a triangulation. The vertices on the extra level are labelled in such a way that the artificial set on this level is in some sense "completely labelled" yielding a fixed point of a linear function. From this set on a unique path of adjacent polyhedra (simplices) can be generated with common "completely labelled" facets. It will appear that if this path terminates the face of the terminal polyhedron (simplex) on the natural level yields an approximate fixed point. Actually, the intersection of the path of polyhedra (simplices) with the natural level is just the sequence of simplices of variable dimension of the algorithm. Hence, the algorithms presented in the previous chapters can be viewed (in case of vector labelling) as tracing zeroes of a piecewise linear homotopy function $\psi(x, \delta)$ from the artificial level $(\delta=0)$ to the natural level $(\delta=1)$. For the case $\mathrm{N}=1$ the interpretations were found independently by Van der Laan and Talman [1979a,b], Todd [1978b] and Bárány [private communication] (see also Van der Laan and Talman [1980c] and Van der Laan [1980]). In case $N=n$ the first interpretation on $R^{n}$ was discovered by Todd and Wright [1979] (see also Todd [1980]). Based on their work Van der Laan and Talman [1979c] developed the three interpretations to be discussed in this chapter. 
Section 2 presents the first interpretation of the variable dimension algorithm on $S$ discussed in chapter 6 . In this section we subdivide the set $S \times[0,1]$ into polyhedra, where the zero-level will denote the artificial level and the one-level the natural one. The second interpretation on $\mathrm{S}$ will be discussed in section 3. For this interpretation the number of artificial vertices is equal to the number of variables. Moreover, these points form the vertices of a simplex. This has the advantage that we obtain a triangulation. The third interpretation is closely related to the second one. Whereas the number of artificial vertices is the same, they now yield a polytope having the same dimension as S. This interpretation will also be discussed in section 3. Finally, section 4 modifies the three interpretations for the class of algorithms on $\mathrm{R}^{\mathrm{n}}$ discussed in chapter 7 .

8.2. INTERPRETATION WITH THE SET S ON THE ZERO-LEVEL.

In this section we give a geometric interpretation of the variable dimension algorithm on the product space $s$ of $N$ unit simplices $s{ }^{m_{j}}$, $j=1, \ldots, N$, by subdividing the set $s \times[0,1]$ into polyhedra. For given grid size vector $d$ and starting point $y$ let $S$ be triangulated into simplices as described in section 6.2. Then we subdivide the set $S \times[0,1]$ as follows, where $u(p)=u\left(p_{1}, \ldots, p_{N}\right)$ denotes the vertex of $s$ with $u_{j, h}(p)=1$ when $h=p_{j}$ and $u_{j, h}(p)=0$ elsewhere, $1 \leq p_{j} \leq m_{j}+1, j \in I_{n}$. Let the regions $\AA(T)$ be defined as in section 6.2. For $T \in Z$, each gridpoint $\mathbf{x} \in \AA(T)$ on the one-level is connected with all the vertices $u(p)$ with $\left(j, p_{j}\right) \notin T$ for all $j \in I_{N}$. on the zero-level. In particular, $(y, 1)$ is connected with all vertices of $S$ on the zero-level. Moreover, a grid point $\mathrm{x} \in \AA(T), T \in \mathrm{z}^{1}$, is connected with only one vertex on the zerolevel, viz. the vertex $u\left(p_{1}, \ldots, p_{N}\right)$ where $\left\{\left(j, p_{j}\right) \mid j=1, \ldots, N\right\}$ is the set of indices not in $T$. Note that in the latter case an $(M+1)$-dimensional simplex is formed when we consider an M-simplex $\sigma\left(y^{1}, \pi(T)\right)$ of the triangulation $G(T)$ of $A(T)$ on the one-level. If $T \notin z^{1}$, then a simplex $\sigma\left(y^{1}, \pi(T)\right)$ of $G(T)$ yields a polyhedron. Recall that if $\sigma\left(y^{1}, \pi(T)\right)$ is a simplex of $G(T)$, then $y^{t+1}$ is a proper point of $A(T)$, i.e. $y^{t+1} \in \AA(T)$. Following the arguments of Todd and wright [1979] we now prove that the just defined collection $L$ of polyhedra indeed subdivides $S \times[0,1]$. For $T \in z$, let $E(T)$ denote the set of vertices $u\left(k_{1}, \ldots, k_{N}\right)$ of $S$ on the 
zero-level such that $\left(j, k_{j}\right) \notin T$ for all $j \in I_{N}$.

THEOREM 8.2.1. Each point $(x, \delta)$ of $S x[0,1]$ lies in a polyhedron of $I$, and the intersection of two polyhedra of $L$ is a face of each.

PROOF. Let $T$ be the unique element of $z$ such that $x \in \stackrel{\circ}{\mathrm{A}}(\mathrm{T})$. Clearly, for any $(j, k) \in T$ there are unique numbers $\lambda_{j, k^{\prime}} \alpha_{j, k}(i, h):(i, h) \in T^{\prime}=T \backslash\{(j, k)\}$, and nonnegative numbers $\beta_{j, k}(p)$ for $u(p) \in E(T)$ such that

$$
x=\lambda_{j, k^{y}}+\underset{(i, h) \in T^{\prime}}{\sum} \alpha_{j, k}(i, h) q(i, h)+\sum_{u(p) \in E(T)} \beta_{j, k}(p) u(p) .
$$

Since $x \in \AA(T)$ we must have that $\lambda_{j, k} \leq 1$. Let $\bar{T}$ be a subset of $T$ such that $(j, k) \in \bar{T}$ if $\lambda_{j, k}<\delta$ and $(j, k) \notin \bar{T}$ if $\lambda_{j, k}>\delta$. Observe that

$\lambda_{j, k}=x_{j, k} / y_{j, k}$ if $y_{j, k}>0$ and $(j, k-1) \notin T$, where $k-1=m_{j}+1$ if $k=1$. It is easily seen that the affine hull of the elements of $E(T)$ and $(x, \delta)$ intersects the region $A(\bar{T})$ on the one-level in a single point, say $(\bar{x}, 1)$. Recall that the rank of the matrix with columns $q(i, h),(i, h) \in T$, is equal to $|\mathrm{T}|$ when $\mathrm{T} \in \mathrm{z}$. Let $\sigma\left(\mathrm{y}^{1}, \pi(\overline{\mathrm{T}})\right)$ be a $\overline{\mathrm{t}}$-simplex of $\mathrm{G}(\overline{\mathrm{T}})$ containing $\bar{x}$, where $\bar{t}=|\bar{T}|$. Then $(x, \delta)$ lies in the polyhedron $P\left(\sigma\left(y^{1}, \pi(\bar{T})\right)\right)$ of $L$ with as vertices the elements of $E(\bar{T})$ and the vertices of $\sigma$ on the one-level. This proves the first part of the theorem.

Next, suppose $P_{1}$ and $P_{2}$ are two polyhedra in $L$ containing $(x, \delta)$ and let $\sigma_{1}$ and $\sigma_{2}$ be the corresponding faces on the natural level, i.e. $\sigma_{i} \times\{1\}=P_{i} \cap(S \times\{1\}), i=1,2$. Then $(x, \delta)$ is contained in the subpolyhedron with as vertices the elements of $E\left(T^{*}\right)$ and the vertices of the face $\sigma_{1} \cap \sigma_{2}$ on the one-level, where $T^{*}$ is the largest subset $\bar{T}$ of $T$ defined above, i.e. $(j, k) \in T^{*}$ if and only if $\lambda_{j, k} \leq \delta$. Clearly, this polyhedron is a common face of both $P_{1}$ and $P_{2}$.

Let $f$ be a continuous function from $S$ into itself. We now show that the path of simplices of variable dimension generated by the algorithm with vector labelling,described in chapter 6 , corresponds to a unique path of adjacent polyhedra in $L$ such that the common facets contain a zero of the piecewise linear homotopy function $\psi(x, \delta)$ obtained by setting 


$$
\psi(x, 0)=C x-e \quad x \in S
$$

and

$$
\psi(x, 1)=x-f(x) \quad x \in S(d),
$$

where $S(d)$ is the set of grid points of $S$ and where $C$ is the diagonal $(M+N) x(M+N)$ matrix whose $(j, k)$-th diagonal element is equal to $\mathrm{m}_{j}+1$, and extending $\psi(x, \delta)$ linearly on each piece of $L$. Since $\psi(x, \delta)$ is linear on the zero-level, it is not hard to check that $\psi$ is linear on each polyhedron of L (cf. Todd and Wright [1979,page 22]). In the next theorem we prove for a facet $\tau\left(w^{1}, \ldots, w^{t}\right)$ of the triangulation $G(T)$ of $A(T), T \in Z$, that $\tau$ is $T$-complete if and only if $\psi$ contains a zero in the polyhedron $P(\tau)$, where $P(\tau)$ is the polyhedron with vertices the elements of $E(T)$ and the vertices of $\tau$ on the natural level.

Therefore, each vertex $u(p)$ of $S$ on the zero-level is labelled by $\ell(u(p), 0)$, where

$$
\ell(u(p), 0)=C u(p) .
$$

Each point $\mathrm{x} \in \mathrm{S}$ on the natural one-level receives a label according to the standard vector labelling rule on $S$. Note that for all $j \in I_{N}$

$$
\ell_{j, k}(u(p), 0)=m_{j}+1 \text { if } k=p_{j}
$$

and

$$
\ell_{j, k}(u(p), 0)=0 \quad \text { if } k \neq p_{j}
$$

THEOREM 8.2.2. Let $\tau\left(\mathrm{w}^{1}, \ldots, \mathrm{w}^{t}\right)$ be a $(t-1)$-facet of $G(T), T \in z$. Then the system of linear equations

$$
\sum_{i=1}^{t} \lambda_{i} l\left(w^{i}\right)+\sum_{(j, k) \notin T} \mu_{j, k} e(j, k)=e
$$


has a (unique) nonnegative solution $\left(\lambda^{*}, \mu^{*}\right)$ if and only if the system of linear equations

$$
\text { (8.2.2) } \sum_{i=1}^{t} \tilde{\lambda}_{i} \ell\left(w^{i}\right)+\sum_{u(p) \in E(T)} v(u(p)) C u(p)=e
$$

has at least one nonnegative solution $\left(\tilde{\lambda}^{*}, \nu^{*}\right)$.

PROOF. Assume that (8.2.2) has a feasible solution $\left(\tilde{\lambda}^{*}, \nu^{*}\right)$. Let $\lambda_{i}^{*}=\tilde{\lambda}_{i}^{*}$ for all $i$, and define $\mu_{j, k}^{*}$ by

$$
\mu_{j, k}^{*}=\left(m_{j}+1\right) \sum_{u(p) \in E(T)} \nu^{*}(u(p)) .
$$

Then $\left(\lambda^{*}, \mu^{*}\right)$ is a feasible solution of (8.2.1). Since the $\tilde{\lambda}_{i}^{*}$ 's are unique as follows from the $(j, k)$-th equations for $(j, k) \in T,\left(\lambda^{*}, \mu^{*}\right)$ must also be unique.

Conversely, let $\left(\lambda^{*}, \mu^{*}\right)$ be the unique feasible solution of (8.2.1). Let $D\left(\lambda^{*}, \mu^{*}\right)$ be the set defined by

$$
\begin{aligned}
& \mathrm{D}\left(\lambda^{*}, \mu^{*}\right)=\left\{(\tilde{\lambda}, \nu) \mid \tilde{\lambda}=\lambda^{*} \text { and } \underset{u(p) \in E(T)}{\sum_{u(p}} \nu(\mathrm{u}(\mathrm{p}))=\mu_{j, \mathrm{k}}^{*}\left(\mathrm{~m}_{j}+1\right)^{-1}\right. \\
& \left.\mathrm{p}_{j}=\mathrm{k} \text { with } \nu \geq 0,(j, k) \notin \mathrm{T}\right\} \text {. }
\end{aligned}
$$

Clearly, $D\left(\lambda^{*}, \mu^{*}\right)$ is nonempty and each $\left(\lambda^{*}, \nu^{*}\right) \in D\left(\lambda^{*}, \mu^{*}\right)$ is a feasible solution of $(8.2 .2)$.

COROLLARY 8.2.3. If for $T \in z$ a simplex $\tau\left(w^{1}, \ldots, w^{t}\right)$ of $G(T)$ is $T$-complete, then for each $\left(\hat{\lambda}^{*}, \nu^{*}\right) \in D\left(\lambda^{*}, \mu^{*}\right)$ the point $\left(x^{*}, \delta^{*}\right)$ is a zero-point of $\psi$ in $P(\tau)$, where

$$
x^{*}=\sum_{i=1}^{t} \cdot \tilde{\lambda}_{i}^{*} w^{i}+\sum_{u(p) \in E(T)} v^{*}(u(p)) u(p)
$$

and

$$
\delta^{*}=\sum_{i=1}^{t} \tilde{\lambda}_{i}^{*}
$$

It is easily verified that 


$$
\sum_{i=1}^{t} \tilde{\lambda}_{i}^{*}+\sum_{u(p) \in E(T)} \nu^{*}(u(p))=1
$$

Observe that $\Sigma \nu^{*}(u(p)) u(p)=\sum \mu_{j, k}^{*} e(j, k) /\left(m_{j}+1\right)$ so that $x^{*}$ is independent of the solution $\left(\tilde{\lambda}^{*}, \nu^{*}\right)$. Consequently, the variable dimension algorithm can be interpreted as a method which generates a unique path of adjacent polyhedra in $L$ such that the common facets contain a zero-point of $\psi$. The method starts with the polyhedron $P(\{y\})=\operatorname{conv}(S \times\{0\} \cup\{(y, 1)\})$. The facet $S \mathrm{x}\{0\}$ of $\mathrm{P}(\{\mathrm{y}\})$, which is a boundary-facet of $\mathrm{S} \times[0,1]$, contains the trivial zero-point $\left(C^{-1} e, 0\right)$ of $\psi$. As soon as a zero-point of $\psi$ in $S \mathrm{x}\{1\}$ is generated, all $\nu^{*}$ become equal to zero and the method terminates. It is easily seen from theorem 8.2.2 that the intersection of the path of generated polyhedra with the natural level is the path of simplices of the variable dimension algorithm described in chapter 6 .

\subsection{TWO INTERPRETATIONS WITH M+N POINTS.}

To give a geometric interpretation of the variable dimension algorithm on $\mathrm{S}$ yielding only simplices, we triangulate the set

$$
\tilde{S}=\operatorname{conv}\left(\left(S^{M+N-1} \times\{0\}\right) \cup(S \times\{1\})\right) .
$$

So, we have only $M+N$ vertices on the artificial level. As in the previous section $S$ is triangulated on the natural level for some grid size vector $d$ and starting point $\mathrm{y}$.

Let $\tilde{G}$ be the set of $(M+N)$-simplices which are obtained by connecting each grid point $x$ in $A(T)$ on the one-level with all the vertices $e(i, h)$ of $S^{\mathrm{M}+\mathrm{N}-1}$ on the zero-level such that $(i, h) \notin T, T \in Z$. In particular $(y, 1)$ is connected with all vertices of $S^{M+N-1} \times\{0\}$.

THEOREM 8.3.1. The set $\tilde{G}$ is a triangulation of $\tilde{S}$.

PROOF. Let $(v, \delta)$ be a point of $\tilde{S}$. Then there are points $x \in S$ and $\mathrm{z} \in \mathrm{S}^{\mathrm{M}+\mathrm{N}-1}$ such that

$$
\mathrm{v}=\delta \mathrm{x}+(1-\delta) z,
$$


and so $\mathrm{v}-\delta \mathrm{y}=\delta(\mathrm{x}-\mathrm{y})+(1-\delta) \mathrm{z}$.

Since $x$ and $y \in S$, we have $\sum_{k=1}^{m_{j}^{+1}}(x-y)_{j, k}=0$ for all $j \in I_{N}$.

Therefore

$$
\sum_{k=1}^{m_{j}+1}(v-\delta y)_{j, k}=(1-\delta) \sum_{k=1}^{m_{j}+1} z_{j, k} \geq 0 \quad j=1, \ldots, N .
$$

Recall that $\mathrm{z} \in \mathrm{S}^{\mathrm{M}+\mathrm{N}-1}$. Hence, there is a unique index set $\mathrm{T}^{*} \in \mathrm{Z}$ such that

$$
v-\delta y=\sum_{(j, k) \notin T^{*}{ }^{\alpha} j, k} e(j, k)+\sum_{(i, h) \in T^{*}}{ }^{\beta} i, h^{q(i, h)}
$$

for unique positive $\alpha_{j, k}$ and nonnegative $\beta_{i, h}$.

Let $T$ be the subset of $T^{*}$ such that $(i, h) \in T$ if $\beta_{i, h}>0$ and define $\alpha_{j, k}=0$ for all $(j, k) \in T^{*} \backslash T$. Then we obtain

$$
v-\delta y=\sum_{(j, k) \notin T}^{\sum} \alpha_{j, k} e^{e(j, k)+\sum_{(i, h) \in T} \beta_{i, h}}{ }^{q(i, h),}
$$

and so

$$
v=\sum_{(j, h) \notin T} a_{j, k} e(j, k)+\delta \bar{v}
$$

where

$$
\bar{v}=y+\delta^{-1} \sum_{(i, h) \in T} \beta_{i, h^{q}}(i, h)
$$

Observe that $\sum \alpha_{j, k}=1-\delta$. Since all $\beta_{i, h}$ 's are positive, we have that $\bar{v} \in \AA(T)$. Let $\sigma\left(y^{1}, \pi(T)\right)$ be a simplex of the triangulation $G(T)$ of $A(T)$ containing $\bar{v}$. Then $(v, \delta)$ lies in the $(M+N)$-simplex with vertices $e(j, k)$, $(j, k) \notin T$, on the zero-level and the vertices of $\sigma$ on the one-level. This proves that the union of all simplices is equal to $\tilde{S}$.

Finally, let $\sigma_{1}$ and $\sigma_{2}$ be two $(M+N)$-simplices of $\tilde{G}$ both containing $(v, \delta)$, and let $\tau_{i}=\sigma_{i} \cap(s \times\{1\}), i=1,2$. Then $(x, \delta)$ is contained in the face of the triangulation $G$ with vertices $e(j, k),(j, k) \notin T^{*}$, on the zero level and the vertices of $\tau_{1} \cap \tau_{2}$ on the one-level. Clearly, this face is 
a common face of both $\sigma_{1}$ and $\sigma_{2}$, which proves the theorem.

The artificial vertices of $\mathrm{S}^{\mathrm{M}+\mathrm{N}-1}$ on the zero-level are labelled as follows:

$$
\ell(e(j, k), 0)=(M+N) e(j, k) \quad(j, k) \in I(M) .
$$

Clearly, a $(t-1)$-simplex $\sigma\left(w^{1}, \ldots, w^{t}\right)$ of the triangulation $G(T)$ of $A(T)$ is $T$-complete if and only if the sytem of linear equations corresponding to the unique $(\mathrm{M}+\mathrm{N}-1)$-facet $\mathrm{P}(\sigma)$ of $\tilde{G}$ having $\sigma$ as the intersection with the natural level

$$
\sum_{i=1}^{t} \tilde{\lambda}_{i} \ell\left(w^{i}\right)+\sum_{(j, k) \notin T} \tilde{\mu}_{j, k} \ell(e(j, k), 0)=e
$$

has a nonnegative solution $\left(\tilde{\lambda}^{*}, \tilde{\mu}^{*}\right)$. Obviously, if $\left(\lambda^{*}, \mu^{*}\right)$ is the solution of (8.2.1) then $\lambda^{*}=\tilde{\lambda}^{*}$ and $\mu^{*}=(M+N) \mu^{*}$, and reversely. Consequently, if $\sigma\left(w^{1}, \ldots, w^{t}\right)$ is a $T$-complete facet of $G(T)$, then the point $\left(x^{*}, \delta^{*}\right)$, where

$$
x^{*}=\sum_{i=1}^{t} \tilde{\lambda}_{i}^{*} w^{i}+\sum_{(j, k) \notin T} \tilde{\mu}_{j, k}^{*} e(j, k) \text { and } \delta^{*}=\sum_{i=1}^{t} \tilde{\lambda}_{i}^{*}
$$

is a zeropoint in $P(\sigma)$ of the piecewise linear homotopy function $\psi(x, \delta)$, where

$$
\psi(x, 0)=(M+N) x-e \quad x \text { is a vertex of } s^{M+N-1}
$$

and

$$
\psi(x, 1)=x-f(x) \quad x \in S(d) .
$$

Observe that lemma 6.5.3 implies

$$
\sum_{i=1}^{t} \tilde{\lambda}_{i}^{*}+\sum_{(j, k) \notin T} \tilde{\mu}_{j, k}^{*}=1
$$

The variable dimension algorithm discussed in section 6.5 can therefore be interpreted as tracing zeroes of the function $\psi$ starting with the point 
$\mathrm{e} /(\mathrm{M}+\mathrm{N})$ on the artificial level and terminating with an approximate fixed point of $f$ as soon as a zero-point is generated on the natural level. The latter occurs if and only if $\mu^{*}(j, k)=0$ for all $(j, k) \notin T$. Moreover, the intersection of the path of the $(M+N)$-simplices of $G$ containing these zeroes with the natural level is the path of simplices of variable dimension of the algorithm. A closely related interpretation involving also $\mathrm{M}+\mathrm{N}$ artificial points is the following. For $(j, k) \in I(M)$, let $v(j, k)$ be the vertex $y-q(j, k)$, and let $\mathrm{V}$ be the convex hull of all these vertices. Consider now the set

$$
\widetilde{V}=\operatorname{conv}((V \times\{0\}) \cup(S \times\{1\})) .
$$

Each grid point $\mathrm{x}$ of $\AA(\mathrm{T})$ on the natural level is connected with all the vertices $\mathrm{v}(\mathrm{j}, \mathrm{k})$ for $(\mathrm{j}, \mathrm{k}) \notin \mathrm{T}$ on the zero-level. In particular, the starting point $\mathrm{y}$ is connected witi: all vertices of $\mathrm{V} \times\{0\}$. Doing so, we obtain a finite collection of polyh:odra each having $\mathrm{M}+\mathrm{N}+1$ vertices. Clearly, when $T \in Z$, a simplex $\sigma\left(Y^{1}, \pi(T)\right)$ in $G(T)$ on the one-level is the face of only one polyhedron $P(\sigma)$ such that $P(0) \cap V x\{0\}$ is the convex hull of the vertices $v(j, k),(j, k) \notin T$, on the zero-level. However, the collection of polyhedra is neither a triangulation nor a rubdivision of $\widetilde{V}$ (except when $N=1$ ), but if we label the vertex $v(j, k)$ on the zero-level by $(M+N) e(j, k)$ the polyhedron $P(\tau)$ of a $(t-1)$-simplex $\tau$ in $G(T)$ contains a zero of the piecewise linear function $\psi_{\tau}(x, \delta)$ if and only if $\tau$ is a $T$-complete simplex in $G(T)$, where

$$
\psi_{\tau}(x, 0)=(M+N) x-e \quad x=v(j, k),(j, k) \notin T
$$

and

$$
\psi_{\tau}(x, 1)=x-f(x) \quad x \in S(d)
$$

So, although we have neither a subdivision nor a triangulation, from $\mathrm{V} \times\{0\}$ on a unique path of polyhedra can be generated by pivot and replacementsteps.-Clearly, no polyhedron can be revisited. Moreover, the intersection of the path with the natural level is the sequence of simplices of the variable dimension algorithm on $S$. Note that all three interpretations conincide if $\mathrm{N}=1$. We remark that the last two interpretations can be easily adapted for 
integer labelling. For the first interpretation it seems to be rather complicated. However it can be done by labelling facets and by giving a single point more than one label. We will not further discuss this matter. Finally, we remark that the second interpretation makes it possible to apply index and orientation theory. Further research is needed. In the next section we will modify the three interpretations for the class of algorithms on $R^{n}$ presented in chapter 7 .

8.4. MODIFICATION FOR THE ALGORITHM ON $\mathrm{R}^{\mathrm{n}}$.

Let $R^{n}$ be triangulated as described in section 7.2 for given starting point $y$ and numbers $m_{1}, \ldots, m_{N}$ with sum $n$. Moreover, let $f$ be $a$ continuous function from $R^{n}$ to $R^{n}$ and let $g: R^{n} \rightarrow R^{n+N}$ be defined as in section 7.3.

To modify the first interpretation presented in section 2, we first define the artificial set on the zero-level. Let, for $j \in I_{N_{1}^{\prime}}$ $\theta_{j}=\theta_{j}\left(x_{j}^{1}, \pi\left(I_{m_{j}}\right)\right)$ be the $m_{j}$-simplex in $R^{m_{j}}$ with vertices $x_{j}, \ldots, x_{j}{ }^{+1}$ such that

$$
x_{j}^{1}=\left(m_{j}+1\right)\left\{y_{j}-\sum_{i=1}^{m_{j}}\left(m_{j}-i+1\right) p_{j}(i)\right\},
$$

$$
\begin{gathered}
\pi\left(I_{m_{j}}\right)=\left(1, \ldots, m_{j}\right) \text { is the standard permutation of the } \\
\text { elements of } I_{m_{j}}^{\prime}
\end{gathered}
$$

where $y_{j}=\left(y_{j, 1}, \ldots, y_{j, m_{j}}\right)^{T}$ and where $p_{j}(i)$ is the $i-t h$ column of the triangulation matrix $P_{j}$ of $R j$ as defined in section 7.2. Observe that $y_{j}$ is the barycentre of $\theta_{j}$. Finally, let $\theta$ be the product space of the simplices $\theta_{j}$ i.e.

$$
\theta=\prod_{j=1}^{N} \theta_{j} .
$$

Then $\theta$ is a polytope with vertices $u(p)=u\left(p_{1}, \ldots, p_{N}\right)$ in $R^{n}$ where for $p_{i} \in I_{m_{i}+1}$, for all $i$, 


$$
u_{j, k}(p)=x_{j, k}^{p_{j}} \quad k=1, \ldots, m_{j} \text { and } j \in I_{N} \text {. }
$$

The set $\theta$ will be the artificial set on the zero-level. Proceeding along the same lines as in section 2 we obtain a subdivision $L$ of the set

$$
\theta=\operatorname{conv}\left((\theta \times\{0\}) \cup\left(R^{n} \times\{1\}\right)\right)
$$

when grid points $\mathrm{x} \in \stackrel{\circ}{\AA}(\mathrm{T})$ on the natural one-level are connected with all the vertices $u(p) \in E(T)$ on the zero-level for any $T \in Z$, where

$$
E(T)=\left\{u(p) \mid\left(j, p_{j}\right) \notin T \text { for } j=1, \ldots, N\right\}
$$

Note that for $t>0$ the number of grid points in $A(T)$ is unbounded, where $|T|=t$ and $T \in Z$. Hence, the subdivision is not locally finite. However, we still have the property that every compact subset of $\theta$ is covered by a finite number of relative interiors of faces of $L$. Now, each vertex $u(p)$ of $\theta$ on the zero-level is labelled by $l(u(p), 0)$, where for $(j, k) \in I(m)$

$$
\begin{array}{ll}
\ell_{j, k}(u(p), 0)=m_{j}+1 & \text { if } k=p_{j} \\
\ell_{j, k}(u(p), 0)=0 & \text { if } k \neq p_{j} .
\end{array}
$$

Of course, a point $(x, 1) \in R^{n} x\{1\}$ receives the label $\ell(x)$ according to the labelling rule defined in section 7.5. Following the same arguments as in section 2 , it is easy to see that a $(t-1)-\operatorname{simplex} \sigma\left(w^{1}, \ldots, w^{t}\right)$ in $\mathrm{G}(\mathrm{T})$ is $\mathrm{T}$-complete if and only if the system of $\mathrm{n}+\mathrm{N}$ linear equations with respect to the polyhedron $P(\sigma)$ having only $\sigma$ as face on the natural level, has a nonnegative solution. More precisely, the sytem of linear equations

$$
\sum_{i=1}^{t} \lambda_{i} l\left(w^{i}\right)+\sum_{(j, k) \notin T} \mu(j, k) e(j, k)=e
$$

has a nonnegative solution $\left(\lambda^{*}, \mu^{*}\right)$ if and only if the system

$$
\sum_{i=1}^{t} \tilde{\lambda}_{i} \ell\left(\left(w^{i}, 1\right)\right)+\sum_{u(p) \in E(T)} v(u(p)) l(u(p), 0)=e
$$


has at least one solution $\left(\tilde{\lambda}^{*}, \nu^{*}\right)$. Again we have $\lambda^{*}=\tilde{\lambda}^{*}$ and

$$
\mu_{j, k}^{*}=\left(m_{j}+1\right) \sum_{\substack{u(p) \in E(T) \\ p_{j}=k}} \nu^{*}(u(p)) \quad \text { for all }(j, k) \notin T .
$$

Moreover, the point $\left(\mathrm{x}^{*}, \delta^{*}\right)$ defined by

$$
x^{*}=\sum_{i=1}^{t} \lambda_{i}^{*} w^{i}+\sum_{u(p) \in E(T)} \nu^{*}(u(p)) u(p)
$$

and

$$
\delta^{*}=\sum_{i=1}^{t} \lambda_{i}^{*}
$$

is a zero-point of the piecewise linear homotopy function $\psi$ with respect to the subdivision where $\psi$ is obtained by setting

$$
\psi(x, 0)=\ell(x, 0)-e \quad x \text { is a vertex of } \theta
$$

and

$$
\psi(x, 1)=g(x) \quad x \text { is a grid point }
$$

end extending linearly on each piece of $\theta$.

Therefore, the algorithmcan be seen as tracing a path of zeroes of $\psi$ starting with the zero-point $(y, 0)$ and terminating as soon as a zero-point on the natural level is found.

The second interpretation cannot be directly applied to the class of algorithms on $R^{n}$. Observe, however, that a point $x_{j} \in R^{m_{j}}$ corresponds to the point $\tilde{x}_{j}$ in the set

$$
w^{m_{j}}=\left\{z_{j} \in R^{m_{j}+1} \mid \sum_{k=1}^{m_{j}+1} z_{j, k}=0\right\},
$$

where

$$
\tilde{x}_{j}=\left(\left(x_{j}\right)^{\top},-\sum_{k=1}^{m_{j}} x_{j, k}\right)^{\top} \text {. }
$$

Then the function $g: R^{n} \rightarrow R^{n+N}$ is equivalent to the function $\tilde{g}$ from 
$w=\Pi_{j=1}^{N} w^{m_{j}}$ into itself if we define

$$
\tilde{g}(\tilde{x})=g(x) \quad \text { for all } \tilde{x} \in W \text {. }
$$

Clearly, $x^{*}$ is a zero-point of $g$ if and only if $\mathrm{x}^{*}$ is a zero-point of $\tilde{g}$. The set $\mathrm{W}$ is now triangulated in the same way as described in section 6.2 for the product space of simplices by using the block-diagonal matrix $\widetilde{P}$ whose $j$-th block $\widetilde{P}_{j}$ is equal to the $\left(m_{j}+1\right) \times\left(m_{j}+1\right)$ extended matrix for which the first $m_{j}$ rows are equal to $P_{j}$ and the last row is given by $-e^{\top} P_{j}$. If $P_{j}$ is of the $\left(\alpha_{j}, \beta_{j}\right)$-form we can take the set $-s^{n+N-1}$ as the artificial set on the zero-level. Then the set $\bar{w}$ defined by

$$
\bar{W}=\operatorname{conv}\left(\left(-S^{n+N-1} \times\{0\}\right) \cup(W \times\{1\})\right)
$$

is triangulated by connecting a grid point $x$ in int $\tilde{A}(T)$ for $T \epsilon z$ on the natural level with all the vertices $(-e(i, h), 0)$ for $(i, h) \notin T$, where

$$
\begin{aligned}
\tilde{A}(T)=\left\{\tilde{x} \in w \mid \tilde{x}=\tilde{y}+\sum_{(j, k) \in T} \lambda_{j, k} \tilde{p}(j, k)\right. \text { for } \\
\text { nonnegative numbers } \left.\lambda_{j, k}\right\} \text {. }
\end{aligned}
$$

By this way the algorithm can be again interpreted as tracing zeroes of the piecewise linear homotopy function $\psi$ where

$$
\psi(x, 0)=-(n+N) x-e \quad x \text { is a vertex of }-s^{n+N-1}
$$

and

$$
\psi(\tilde{x}, 1)=\tilde{g}(\tilde{x}) \quad \tilde{x} \text { is a vertex of } w \text {. }
$$

To do so, we label a point $x$ on the zero-level by $\ell(x, 0)=-(n+N) x$, whereas $\ell(\tilde{x}, 1)=\ell(x)$ if $\tilde{x}$ is a point in $w$. Clearly, a $(t-1)$-simplex $\sigma\left(w^{1}, \ldots, w^{t}\right)$ in $G(T), T \in Z$, is $T$-complete if and only if the system of linear equations corresponding to the (unique) simplex $\tau$ having $\sigma\left(w^{1}, \ldots, w^{t}\right)$ as its intersection with the one-level, has a feasible solution. This solution gives us the zero-point of $\psi$ in $\tau$, etc.. 
The third interpretation is straightforward by taking $\mathrm{y}-\mathrm{p}(\mathrm{j}, \mathrm{k})$ for $(j, k) \in I(m)$ as the artificial vertices on the zero-level. Observe that the three interpretations again coincide for $\mathrm{N}=1$. Based upon these coinciding interpretations in case $\mathbf{N}=1$, Van der Laan and Talman [1980b] and Shamir [1979] developed their continuous-deformation algorithms on $s^{\mathrm{n}}$ and $\mathrm{R}^{\mathrm{n}}$. It is an interesting question whether such an algorithm can be developed on $S$ or $R^{n}$ if $N>1$. If so, then this opens the possibility to develop a continuous-deformation algorithm generating an infinite path of adjacent simplices of variable dimension converging to a fixed point. Another interesting problem is the orientation of a simplex. For $N=1$ the orientation theory of Eaves and Scarf [1976] and others can directly be applied but for $\mathrm{N}>1$ this is not clear. However, the second interpretation seems to be suitable for generalizing the concept of orientation used in the way as described by Van der Laan [1980, chapter 7] for the case $N=1$. Finally, we mention that it is still an open question how to obtain an estimate of the number of stopping simplices if $\mathrm{N}>1$. This problem can probably be solved as soon as the orientation-problem is solved. 


\section{REF'ERENCES}

[1] Allgower, E.L. and Georg, K. (1980), Simplicial and continuation methods for approximation of fixed points and solutions to systems of equations, SIAM Rev. 22, 28-75.

[2] Bárány, I. (1979), Subdivisions and triangulations in fixed point algorithms, Int.Res.Inst.Man.Sci., ul. Ryleeva, Preprint, Moscow, U.S.S.R.

[3] Berge, C. (1966), Espace topologiques, Dunod, Paris, France.

[4] Brouwer, L.E.J. (1912), Über Abbildung von Mannigfaltigkeiten, Math. Ann. 71, 97-115.

[5] Cottle, R.W. (1966), Nonlinear programs with positively bounded Jacobians, SIAM J.Appl.Math. 14, 147-158.

[6] Eaves, B.C. (1970), An odd theorem, Proc.Amer.Math.Soc. 26, 509-513.

[7] Eaves, B.C. (1971), Computing Kakutani fixed points, SIAM J.Appl. Math. 21, 236-244.

[8] Eaves, B.C. (1972), Homotopies for computation of fixed points, Math. Programming $\underline{3}, 1-22$.

[9] Eaves, B.C. (1976), A short course in solving equations with PL homotopies, SIAM-AMS Proceedings 9, 73-143.

[10] Eaves, B.C. and Saigal, R. (1972), Homotopies for computation of fixed points on unbounded regions, Math.Programming $\underline{3}$, 225-237.

[11] Eaves, B.C. and Scarf, H.E. (1976), The solution of systems of piecewise linear equations, Math.Oper.Res, 1, 1-27.

[12] Fisher, M.L., Gould, F.J. and Tolle, J.W. (1977), A new simplicial approximation algorithm with restarts: relations between convergence and labellings, Fixed points: algorithms and applications, S. Karamardian, ed., Academic Press, New York, pp. 41-58.

[13] Forster, w. (1980), Fixed point algorithms and array processors, Numerical solution of highly nonlinear problems, W.Foster, ed., North-Holland, Amsterdam, The Netherlands, pp. 169-179.

[14] Freudenthal, H. (1942), Simplizialzerlegungen von beschränkter Flachheit, Ann. of Math. $\underline{43}, 580-582$. 
[15] Garcia, C.B. and Gould, F.J. (1976), An algorithm based on the equivalence of vector and scalar labels in simplicial approximation, Center for Mathematical Studies in Business and Economics Report 7626, University of Chicago, Ill..

[16] Garcia, C.B. and Gould, F.J. (1978), A theorem on homotopy paths, Math. Oper.Res. 3 , 282-289.

[17] Garcia, C.B. and Gould, F.J. (1979), Scalar labelling for homotopy paths, Math. Programming 17, 184-197.

[18] Garcia, C.B., Lemke, C.E. and Lüthi, H.J. (1973), Simplicial approximation of an equilibrium point for noncooperative N-person games, Math. Programming, T.C. Hu and S.M. Robinson, eds., Academic Press, New York, pp. 227-260.

[19] Gould, F.J. and Tolle, J.w. (1974), A unified approach to complementarity in optmization, Discrete Math. ㄱ. 225-271.

[20] Gould, F.J. and Tolle, J.W. (1975), Finite and constructive conditions for a solution to $f(x)=0$, Center for Mathematical Studies in Business and Economics Report 7515, University of Chicago, Chicago, Ill..

[21] Hansen, T. (1968), On the approximation of a competitive equilibrium, Ph.D.thesis, Yale University, New Haven, Conn..

[22] Heyden, L. van der (1979), Refinement methods for computing fixed points using primitive sets, Ph.D.thesis, Yale University, New Haven, Conn. .

[23] Kakutani, s. (1941), A generalization of Brouwer's fixed point theorem, Duke Math. J. $\underline{8}, 457-459$.

[24] Kellogg, R.B., Li, T.Y. and Yorke, J. (1976), A constructive proof of the Brouwer fixed point theorem and computational results, SIAM J.Numer. Anal. $\underline{4}, 473-483$.

[25] Kojima, M. (1978a), On the homotopic approach to systems of equations with separable mappings, Math. Programming stud. I, 170-184.

[26] Kojima, M. (1978b), A modification of Todd's triangulation $J_{3}$, Math. Programming 15, 223-227.

[27] Kojima, M. (1979), A note on "A new algorithm for computing fixed points" by Van der Laan and Talman, Numerical solution of highly nonlinear problems, W.Foster, ed., North-Holland, Amsterdam, The Netherlands, pp. 37-42. 
[28] Kuhn, H.W. (1960), Some combinatorial lemmas in topology, IBM J.Res. Develop. $\underline{4}, 518-524$.

[29] Kuhn, H.W. (1968), Simplicial approximation of fixed points, Proc.Nat.Acad.Sci. U.S.A. 61, 1238-1242.

[30] Kuhn, H.W. (1969), Approximate search for fixed points, Computing methods in optimization problems 2, Academic Press, New York.

[31] Kuhn, H.W. and Mackinnon, J.G. (1975), Sandwich method for finding fixed points. J. Optimization Theory Appl. 17, 189-204.

[32] Laan, G. van der (1980), Simplicial fixed point algorithms, Dissertation, Vrije Universiteit, Amsterdam, The Netherlands.

[33] Laan, G. van der and Talman, A.J.J. (1978a), On the computation of fixed points in the product space of unit simplices and an application to noncooperative $N$-person games, IAWE, onderzoekverslag 35, Vrije Universiteit, Amsterdam, The Netherlands.

[34] Laan, G. van der and Talman, A.J.J. (1978b), A class of simplicial subdivisions for restart fixed point algorithms without an extra dimension, IAWE, onderzoekverslag 36, vrije Universiteit, Amsterdam, The Netherlands, to appear in Math. Programming.

[35] Laan, G. van der and Talman, A.J.J. (1979a), A restart algorithm for computing fixed points without an extra dimension. Math. Programming 17, 74-84.

[36] Laan, G. van der and Talman, A.J.J. (1979b), A restart algorithm without an artificial level for computing fixed points on unbounded regions, Functional differential equations and approximation of fixed points, H.-O. Peitgen and H.-O. Walther, eds., Lecture Notes in Mathematics 730 , Springer, Berlin, West Germany, pp. 247-256.

[37] Laan, G. van der and Talman, A.J.J. (1979c), Interpretation of the variable dimension fixed point algorithm with an artificial Zevel, IAWE, onderzoekverslag 47, Vrije Universiteit, Amsterdam, The Netherlands. 
[38] Laan, G. van der and Talman, A.J.J. (1980a), An improvement of fixed point algorithms by using a good triangulation, Math. Programming 18, 274-285.

[39] Laan, G. van der and Talman, A.J.J. (1980b), A new subdivision for computing fixed points with a homotopy algorithm, Math. Programming 19, 78-91.

[40] Laan, G. van der and Talman, A.J.J. (1980c), Convergence and properties of recent variable dimension algorithms, Numerical solution of highly nonlinear problems, W.Foster,ed., North-Holland, Amsterdam, The Netherlands, pp. 3-36.

[41] Lefschetz, S. (1949), Introduction to topology, Princeton University Press, Princeton, N.J..

[42] Lemke, C.E. (1965), Bimatrix equilibrium points and mathematical progromming, Management 'sci. 11, 681-689.

[43] Lemke, C.E. and Grotzinger, S.J. (1976), On generalizing Shapley's index theory to labelled pseudo manifolds, Math. Programming 10, 245-262.

[44] Lemke, C.E. and Howson Jr., J.T. (1964), Equilibrium points of bimatrix gomes, SIAM J.Appl.Math. 12, 413-423.

[45] Lüthi, H.J. (1975), A simplicial approximation of a solution for the nonlinear complementarity problem, Math.Programming 9 , 278-293.

[46] Lüthi, H.J. (1976), Komplementaritäts- und Fixpunktalgorithmen in der mathematischen Progromierung, Spieltheorie und Okonomie, Lecture Notes in Economics and Mathematical Systems 129, Springer, Berlin, West Germany.

[47] MacKinnon, J.G. (1975), An algorithm for the generalized transportation problem, Regional Sci. and Urban Econom. $\underline{5}, 445-464$.

[48] MacKinnon, J.G. (1976), A technique for the solution of spatial equilibrium models, J.Regional Sci. 16, 293-307.

[49] Mara, P.S. (1972), Triangulations of a cube, M.s. thesis, Colorado State University, Fort Collins, Colo.. 
[50] Merril, О.H. (1971), Applications and extensions of an algorithm that computes fixed points of certain nonempty, convex, upper semi-continuous point to set mappings, Department of Industrial Engineering, Preprint 71-7, University of Michigan, Ann Arbor, Mich..

[51] Merrill, O.H. (1972), Applications and extensions of an algorithm that computes fixed points of certain upper semi-continuous point to set mappings, Ph.D. thesis, University of Michigan, Ann Arbor, Mich..

[52] Moré, J. (1974), Coercivity conditions in nonlinear complementarity problems, SIAM Rev. 16, 1-16.

[53] Owen, G. (1968), Game Theory, W.B. Saunders Company, Philadelphia, Pa. .

[54] Reiser, P.M. (1978a), Ein hybrides Verfahren zur Lösung von nichtlinearen Komplementaritätsproblemen und seine Konvergenzeigenschaften, Dissertation, Eidgenössischen Technischen Hochschule Zürich, Zürich, Switzerland.

[55] Reiser, P.M. (1978b), A modified integer. labelling for complementarity algorithms, Institut für Operations Research, Manuskripte, Universität zürich, Zürich, Switzerland.

[56] Reiser, P.M. (1978c), Convergence properties of complementarity algorithms with integer labelling, Institut für operations Research, Manuskripte, Universität zürich, zürich, Switzerland.

[57] Rockafellar, R.T. (197.0), Convex Analysis, Princeton University Press, Princeton, N.J..

[58] Saari, D.G. and Saigal, R. (1979), Some generic properties of paths generated by fixed point algorithms, Department of Industrial Engineering and Management Science, Preprint, Northwestern University, Evanston, Ill., to appear in the Proceedings of the Symposium on Analysis and Computation of Fixed Points, Madison, Wi..

[59] Saigal, R. (1976), On paths generated by fixed point algorithms, Math.Oper.Res. 1, 359-380. 
[60] Saigal, R. (1977a), Investigations into the efficiency of fixed point algorithms, Fixed points: algorithms and applications, S. Karamardian, ed., Academic Press, New York, pp. 203-223.

[61] Saigal, R. (1977b), Fixed point computing methods, Encyclopedia of Computer Sci. and Technology $\underline{8}, 213-235$.

[62] Saigal, R. (1977c), On the convergence rate of algorithms for solving equations that are based on methods of complementary pivoting, Math.oper.Res. 2 , 108-124.

[63] Saigal, R. (1979), On piecewise linear approximations to smooth mappings, Math.Oper.Res. $\underline{4}, 153-161$.

[64] Saigal, R. and Todd, M.J. (1978), Efficient acceleration techniques for fixed point algorithms, SIAM J.Numer.Anal. 15, 997-1007.

[65] Saigal, R., Solow, D. and Wolsey, L.A. (1975), A comparative study of two algorithms to compute fixed points over unbounded regions, presented at the VIII-th Mathematical Programming Symposium, Stanford, Calif..

[66] Scarf, H. (1976), The approximation of fixed points of a continuous mopping, SIAM J.Appl.ilath. 15, 1328-1343.

[67] Scarf, H.E. (with the collaboration of T. Hansen) (1973), Computation of economic equilibria, Yale University Press, New Haven, Conn. .

[68] Shamir, s. (1979), A homotopy fixed point algorithm with an arbitrary integer refinement factor, Department of Engineering - Economic Systems, Report SE - 79R - 2, Stanford University, Stanford, Calif..

[69] Shapley, I.S. (1973), On balanced gomes without side payments, Mathematical Programming, T.C. Hu and S.M. Robinson, eds., Academic Press, New York, pp. 261-290.

[70] Shapley, L.S., (1974), A note on the Lemke-Howson algorithm, Math. Programming Stud. 1 , 175-189.

[71] Spanier, E.H. (1966), Algebraic topology, MacGraw-Hill, New York.

[72] Sperner, E. (1928), Neuer Beweis für die Invarianz der Dimensionszahl und des Gebietes, Abh.Math.Sem. Univ.Hamburg 6, 265-272.

[73] Stynes, M. (1979), An n-dimensional bisection method for solving systems of $n$ equations in $n$ unknowns, Roinn na Matamaitice, Preprint, Colaiste na hollscoile, Corcaigh, Ireland. 
[74] Todd, M.J. (1976a), The computation of fixed points and applications, Lecture Notes in Economics and Mathematical Systems 124, Springer, Berlin, West Germany.

[75] Todd, M.J. (1976b), On triangulations for computing fixed points, Math. Programming 10, 322-346.

[76] Todd, M.J. (1976c), Orientation in complementary pivot algorithms, Math.Oper.Res. 1 , 54-66.

[77] Todd, M.J. (1977), Union Jack triangulations, Fixed points: algorithms and applications, S. Karamardian, ed., Academic Press, New York, pp. 315-336.

[78] Todd, M.J. (1978a), Improving the convergence of fixed point algorithms, Math. Programming Stuid. ㄱ. 151-169.

[79] Todd, M.J. (1978b), Fixed-point algorithms that allow restarting without an extra dimension, School of operations Research and Industrial Engineering, Technical Report 379, Cornell University, Ithaca, N.Y..

[80] Todd, M.J. (1978c), Exploiting stmucture in fixed-point computation, Mathematics Research Center, Technical Summary Report, University of Wisconsin, Madison, Wi..

[81] Todd, M.J. (1978d), Traversing large pieces of linearity in algorithms that solve equations by following piecewiselinear paths, School of operations Research and Industrial Engineering, Technical Report 390, Cornell University, Ithaca, N.Y..

[82] Todd, M.J. (1978e), On the Jacobian of a function at a zero computed by a fixed point algorithm, Math.Oper.Res. 3 , 126-132.

[83] Todd, M.J. (1980), GLobal and local convergence and monotonicity results for a recent variable-dimension simplicial algorithm, Numerical solution of highly nonlinear problems, w.Foster, ed., North-Holland, Amsterdam, The Netherlands, pp. 43-69.

[84] Todd, M.J. and Acar, R.C. (1978), A note on optimally dissection simplices, School of Operations Research and Industrial Engineering, Technical Report 383, Cornell University, Ithaca, N.Y.. 
[85] Todd, M.J. and Wright, A.H. (1979), A variable-dimension simplicial algorithm for antipodal fixed-point theorems, School of Operations Research and Industrial Engineering, Technical Report 417, Cornell University, Ithaca, N.Y..

[86] Tuy, H. (1979), Pivotal methods for computing equilibrium points: Unified approach and new restart algorithm, Math. Programming, 16, 210-227.

[87] Von Neumann, J. (1937), Uber ein ökonomisches Gleichungs-system und eine Verallgemeinerung des Brouwerschen Fixpunktsatzes, Ergebnisse eines Mathematischen Kolloquiums $\underline{8}$, 73-83.

[88] Whitney, H. (1957), Geometric integration theory, Princeton University Press, Princeton, N.J..

[89] Wolsey, L.A. (1974), Convergence, simplicial paths and acceleration methods for simplicial approximation algorithms for finding a zero of a system of nonlinear equations, CORE Discussion Paper 7427, Heverlee, Belgium.

[90] Wrighi, A.H. (1979), The octahedral algorithm, a new simplicial fixed point algorithm, Department of Mathematics, Preprint, Western Michigan University, Kalamazoo, Mich..

[91] Zangwill, w.I. (1977), An excentric barycentric fixed point algorithm, Math.oper.Res. 2, 343-359. 


\section{OTHER TITLES IN THE SERIES MATHEMATICAL CENTRE TRACTS}

A leaflet containing an order-form and abstracts of all publications mentioned below is available at the Mathematisch Centrum, Kruislaan 413, Amsterdam 1098SJ, The Netherlands. Orders should be sent to the same address.

MCT 1 T. VAN DER WALT, Fixed and almost fixed points, 1963. ISBN 906196 0029 .

MCT 2 A.R. BLOEMENA, Sampling from a graph, 1964 . ISBN 9061960037.

MCT 3 G. DE LEVE, Generalized Markovian decision processes, part I: Model and method, 1964. ISBN 9061960045.

MCT 4 G. DE LEVE, Generalized Markovian decision processes, part II: Probabilistic background, 1964. ISBN 9061960053.

MCT 5 G. DE LEVE, H.C. TIJMS \& P.J. WEEDA, Generalized Markovian decision processes, Applications, 1970. ISBN 9061960517.

MCT 6 M.A. MAURICE, Compact ordered spaces, 1964 . ISBN 9061960061.

MCT 7 W.R. VAN ZWET, Convex transformations of random variables, 1964. ISBN $906196007 \mathrm{x}$.

MCT 8 J.A. ZONNEVELD, Automatic numerical integration, 1964. ISBN 906196 0088.

MCT 9 P.C. BAAYEN, Universal morphisms, 1964. ISBN 9061960096.

MCT 10 E.M. DE JAGER, Applications of distributions in mathematical physics, 1964. ISBN $906196010 \mathrm{x}$.

MCT 11 A.B. PAALMAN-DE MIRANDA, Topological semigroups, 1964. ISBN 906196 0118.

MCT 12 J.A.TH.M. VAN BERCKEL, H. BRANDT CORSTIUS, R.J. MOKKEN \& A. VAN WIJNGAARDEN, Formal properties of newspaper Dutch, 1965. ISBN 9061960134 .

MCT 13 H.A. LAUWERIER, Asymptotic expansions, 1966, out of print; replaced by MCT 54 and 67.

MCT 14 H.A. LAUWERIER, Calculus of variations in mathematical physics, 1966. ISBN 9061960207.

MCT 15 R. DOORNBOS, SZippage tests, 1966. ISBN 9061960215.

MCT 16 J.W. DE BAKKER, Formal definition of programming Zanguages with an application to the definition of ALGOL 60, 1967. ISBN 906196 0223 .

MCT 17 R.P. VAN DE RIET, Formula manipulation in ALGOL 60, part 1, 1968. ISBN 9061960258.

MCT 18 R.P. VAN DE RIET, Formila manipulation in ALGOL 60, part 2, 1968. ISBN $906196038 \mathrm{x}$.

MCT 19 J. VAN DER SLOT, Some properties related to compactness, 1968. ISBN 9061960266 .

MCT 20 P.J. VAN DER HOUWEN, Finite difference methods for solving partial differential equations, 1968. ISBN 9061960274. 
MCT 21 E. WATTEL, The compactness operator in set theory and topology, 1968. ISBN 9061960282.

MCT 22 T.J. DEKKER, ALGOL 60 procedures in numerical algebra, part 1, 1968. ISBN 9061960290.

MCT 23 T.J. DEKKER \& W. HOFFMANN, ALGOL 60 procedures in numerical algebra, part 2, 1968. ISBN 9061960304.

MCT 24 J.W. DE BAKKER, Recursive procedures, 1971. ISBN 9061960606.

MCT 25 E.R. PAERL, Representations of the Lorentz group and projective geometry, 1969. ISBN 9061960398.

MCT 26 EUROPEAN MEETING 1968, Selected statistical papers, part I, 1968. ISBN 9061960312 .

MCT 27 EUROPEAN MEETING 1968, Selected statistical papers, part II, 1969. ISBN 9061960401.

MCT 28 J. DOSTERHOFF, Combination of one-sided statistical tests, 1969. ISBN $906196041 \mathrm{x}$.

MCT 29 J. VERHOEFF, Error detecting decimal codes, 1969. ISBN 9061960428 .

MCT 30 H. BRANDT CORSTIUS, Excercises in computational linguistics, 1970. ISBN 9061960525.

MCT 31 W. MOLENAAR, Approximations to the Poisson, binomial and hypergeometric distribution functions, 1970. ISBN 9061960533.

MCT 32 L. DE HAAN, On regular variation and its application to the weak convergence of sample extremes, 1970. ISBN 9061960541.

мСт 33 F.W. STEUTEL, Preservation of infinite divisibility under mixing and related topics, 1970. ISBN 9061960614.

MCT 34 I. JUHÁSZ, A. VERBEEK \& N.S. KROONENBERG, Cardinal functions in topology, 1971. ISBN 9061960622 .

MCT 35 M.H. VAN EMDEN, An analysis of complexity, 1971. ISBN 9061960630.

MCT 36 J. GRASMAN, On the birth of boundary Zayers, 1971. ISBN 9061960649.

MCT 37 J.W. DE BAKKER, G.A. BLAAUW, A.J.W. DUIJVESTIJN, E.W. DIJKSTRA, P.J. VAN DER HOUWEN, G.A.M. KAMSTEEG-KEMPER, F.E.J. KRUSEMAN ARETZ, W.L. VAN DER POEL, J.P. SCHAAP-KRUSEMAN, M.V. WILKES \& G. ZOUTENDIJK, MC-25 Informatica Symposium, 1971. ISBN 9061960657.

MCT 38 W.A. VERLOREN VAN THEMAAT, Automatic analysis of Dutch compound words, 1971. ISBN 9061960738.

MCT 39 H. BAVINCK, Jacobi series and approximation, 1972. ISBN 9061960746.

MCT 40 H.C. TIJMS, Analysis of $(s, S)$ inventory models, 1972. ISBN 9061960754 .

MCT 41 A. VERBEEK, Superextensions of topological spaces, 1972. ISBN 90 61960762.

MCT 42 W. VERVAAT, Success epochs in Bernoulzi trials (with applications in number theory), 1972. ISBN 9061960770.

MCT 43 F.H. RUYMGAART, Asymptotic theory of rank tests for independence, 1973. ISBN 9061960819.

MCT 44 H. BART, Meromorphic operator vaiued functions, 1973. ISBN 9061960827. 
MCT 45 A.A. BALKEMA, Monotone transformations and Zimit Zaws, 1973. ISBN 9061960835 .

MCT 46 R.P. VAN DE RIET, ABC ALGOI, A portable language for formula manipulation systems, part 1: The language, 1973. ISBN 9061960843.

MCT 47 R.P. VAN DE RIET, $A B C$ ALGOL, A portable Zanguage for formula manipulation systems, part 2: The compiler, 1973. ISBN 9061960851.

MCT 48 F.E.J. KRUSEMAN ARETZ, P.J.W. TEN HAGEN \& H.L. OUDSHOORN, An ALGOL 60 compiler in ALGOL 60, Text of the MC-compiler for the $E L-X 8,1973$. ISBN $906196086 \mathrm{x}$.

MCT 49 H. KoK, Connected orderable spaces, 1974. ISBN 9061960886.

MCT 50 A. VAN WIJNGAARDEN, B.J. MAILLOUX, J.E.L. PECK, C.H.A. KOSTER, M. SINTZOFF, C.H. LINDSEY, L.G.L.T. MEERTENS \& R.G. FISKER (Eds), Revised report on the algorithmic Zanguage ALGOL 68, 1976. ISBN 906196.0894.

MCT 51 A. HORDIJK, Dynamic programming and Markov potential theory, 1974. ISBN 9061960959.

MCT 52 P.C. BAAYEN (ed.), Topological stmuctures, 1974. ISBN 9061960967.

MCT 53 M.J. FABER, Metrizability in generalized ordered spaces, 1974. ISBN 9061960975 .

MCT 54 H.A. LAUWERIER, Asymptotic analysis, part 1, 1974. ISBN 9061960983.

MCT 55 M. HALL JR. \& J.H. VAN LINT (Eds), Combinatorics, part 1: Theory of designs, finite geometry and coding theory, 1974. ISBN 9061960991 .

MCT 56 M. HALL JR. \& J.H. VAN LINT (Eds), Combinatorics, part 2: graph theory, foundations, partitions and combinatorial geometry, 1974. ISBN 9061961009.

MCT 57 M. HALL JR. \& J.H. VAN LINT (Eds), Combinatorics, part 3: Combinatorial group theory, 1974. ISBN 9061961017.

MCT 58 W. ALBERS, Asymptotic expansions and the deficiency concept in statistics, 1975. ISBN 9061961025 .

MCT 59 J.L. MIJNHEER, Sample path properties of stabie processes, 1975. ISBN 9061961076 .

MCT 60 F. GÖBEL, Queueing models involving buffers, 1975. ISBN 9061961084.

* MCT 61 P. VAN EMDE BOAs, Abstract resource-bound classes, part 1. ISBN 9061961092 .

* MCT 62 P. VAN EMDE BOAS, Abstract resource-bound classes, part 2. ISBN 9061961106 .

MCT 63 J.W. DE BAKKER (ed.), Foundations of computer science, 1975. ISBN 9061961114.

MCT 64 W.J. DE SCHIPPER, Symmetric closed categories, 1975. ISBN 906196 1122 .

MCT 65 J. DE VRIES, Topological transformation groups 1 A categorical approach, 1975. ISBN 9061961130 .

MCT 66 H.G.J. PIJLS, Locally convex algebras in spectral theory and eigenfunction expansions, 1976. ISBN 90.61961149. 
* MCT 67 H.A. LAUWERIER, Asymptotic analysis, part 2. ISBN $906196119 \mathrm{x}$.

MCT 68 P.P.N. DE GROEN, Singularly perturbed differential operators of second order, 1976. ISBN 9061961203.

MCT 69 J.K. LENSTRA, Sequencing by enumerative methods, 1977. ISBN 9061961254 .

MCT 70 W.P. DE ROEVER JR., Recursive program schemes: semantics and proof theory, 1976. ISBN 9061961270 .

MCT 71 J.A.E.E. VAN NUNEN, Contracting Markov decision processes, 1976. ISBN 9061961297.

MCT 72 J.K.M. JANSEN, Simple periodic and nonperiodic Lamé functions and their applications in the theory of conical waveguides,1977. ISBN 9061961300 .

MCT 73 D.M.R. IEIVANT, Absoluteness of intuitionistic Zogic, 1979. ISBN $906196122 \mathrm{x}$.

MCT 74 H.J.J. TE RIELE, A theoretical and computational study of generalized aliquot sequences, 1976. ISBN 9061961319.

MCT 75 A.E. BROUWER, Treelike spaces and related connected topological spaces, 1977. ISBN 9061961327.

MCT 76 M. REM, Associons and the closure statement, 1976. ISBN 9061961351.

MCT 77 W.C.M. KALLENBERG, Asymptotic optimality of likelihood ratio tests in exponential families, 1977 ISBN 9061961343.

MCT 78 E. DE JONGE, A.C.M. VAN ROOIJ, Introduction to Riesz spaces, 1977. ISBN 9061961335 .

MCT 79 M.C.A. VAN ZUIJLEN, Empirical distributions and rankstatistics, 1977. ISBN 9061961459.

MCT 80 P.W. HEMKER, A numerical study of stiff two-point boundary problems, 1977. ISBN 9061961467 .

MCT 81 K.R. APT \& J.W. DE BAKKER (Eds), Foundations of computer science II, part 1, 1976. ISBN 9061961408.

MCT 82 K.R. APT \& J.W. DE BAKKER (Eds), Foundations of computer science II, part 2, 1976. ISBN 9061961416.

MCT 83 L.S. VAN BENTEM JUTTING, Checking Landau's "Gmindlagen" in the AUTOMATH system, 1979 ISBN 9061961475.

MCT 84 H.L.L. BUSARD, The translation of the elements of Euclid from the Arabic into Latin by Hermann of Carinthia (?) books vii-xii, 1977. ISBN 9061961483 .

MCT 85 J. VAN MILL, Supercompactness and WaZlman spaces, 1977. ISBN 9061961513 .

MCT 86 S.G. VAN DER MEULEN \& M. VELDHORST, TOrmix I, 1978. ISBN 9061961521 .

* MCT 87 S.G. VAN DER MEULEN \& M. VELDHORST, TOrmix II, ISBN $906196153 \mathrm{x}$.

MCT 88 A. SCHRIJVER, Matroids and Zinking systems, 1977. ISBN 9061961548 . 
MCT 89 J.W. DE ROEVER, Complex Fourier transformation and analytic functionals with unbounded carriers, 1978. ISBN 9061961556.

* MCT 90 L.P.J. GROENEWEGEN, Characterization of optimal strategies in dynamic games, . ISBN 9061961564.

MCT 91 J.M. GEYSEL, Transcendence in fields of positive characteristic, 1979. ISBN 9061961572.

MCT 92 P.J. WEEDA, Finite generalized Markov programing,1979. ISBN 9061961580 .

MCT 93 H.C. TIJMS (ed.) \& J. WESSELS (ed.), Markov decision theory, 1977. ISBN 9061961602 .

MCT 94 A. BIJLSMA, Simultaneous approximations in transcendental number theory, 1978. ISBN 9061961629.

MCT 95 K.M. VAN HEE, Bayesian control of Markov chains, 1978. ISBN 9061961637.

MCT 96 P.M.B. VITÁNYI, Lindenmayer systems: structure, languages, and growth functions, 1980 . ISBN 9061961645.

* MCT 97 A. FEDERGRUEN, Markovian control problems; functional equations and algorithms, . ISBN 9061961653.

MCT 98 R. GEEL, Singular perturbations of hyperbolic type, 1978. ISBN 9061961661

MCT 99 J.K. LENSTRA, A.H.G. RINNOOY KAN \& P. VAN EMDE BOAS, Interfaces between computer science and operations research, 1978. ISBN $906196170 \mathrm{x}$.

MCT 100 P.C. BAAYEN, D. VAN DULST \& J. OOSTERHOFF (Eds), Proceedings bicentennial congress of the Wiskundig Genootschap, part 1,1979. ISBN 9061961688 .

MCT 101 P.C. BAAYEN, D. VAN DULST \& J. OOSTERHOFF (Eds), Proceedings bicentennial congress of the Wiskundig Genootschap, part 2,1979. ISBN 9091961696.

MCT 102 D. VAN DULST, Reflexive and superreflexive Banach spaces, 1978. ISBN 9061961718.

MCT $103 \mathrm{~K}$. VAN HARN, Classifying infinitely divisible distributions by functional equations, 1978 . ISBN 9061961726.

MCT 104 J.M. VAN WOUWE, Go-spaces and generalizations of metrizability,1979. ISBN 9061961734 .

* MCT 105 R. HELMERS, Edgeworth expansions for Zinear combinations of order statistics, . ISBN 9061961742.

MCT 106 A. SCHRIJVER (Ed.), Packing and covering in combinatorics, 1979. ISBN 9061961807.

MCT 107 C. DEN HEIJER, The numerical solution of nonlinear operator equations by imbedding methods, 1979. ISBN 9061961750 .

MCT 108 J.W. DE BAKKER \& J. VAN LEEUWEN (Eds), Foundations of computer science III, part 1, 1979. ISBN 9061961769. 
MCT 109 J.W. DE BAKKER \& J. VAN LEEUWEN (Eds), Foundations of computer science III, part 2, 1979. ISBN 9061961777.

MCT 110 J.C. VAN VLIET, ALGOL 68 transput, part I: Historical Review and Discussion of the Implementation Model, 1979. ISBN 9061961785.

MCT 111 J.C. VAN VLIET, ALGOL 68 transput, part II: An implementation model, 1979. ISBN 9061961793.

MCT 112 H.C.P. BERBEE, Random walks with stationary increments and Renewal theory, 1979. ISBN 906196.1823.

MCT 113 T.A.B. SNIJDERS, Asymptotic optimality theory for testing problems with restricted alternatives, 1979. ISBN 9061961831.

MCT 114 A.J.E.M. JANSSEN, Application of the Wigner distribution to harmonic analysis of generalized stochastic processes, 1979. ISBN $906196184 \mathrm{x}$.

MCT 115 P.C. BAAYEN \& J. VAN MILL (Eds), Topological Structures II, part 1, 1979. ISBN 9061961855 .

MCT 116 P.C. BAAYEN \& J. VAN MILL (Eds), Topological Structures II, part 2, 1979. ISBN 9061961866.

MCT 117 P.J.M. KALLENBERG, Branching processes with continuous state space, 1979. ISBN 9061961882 .

MCT 118 P. GROENEBOOM, Large deviations and Asymptotic efficiencies, 1980. ISBN 9061961904.

MCT 119 F. PETERS, Sparse matrices and substmuctures, 1980. ISBN 906196 1920 .

MCT 120 W.P.M. DE RUYTER, On the Asymptotic Analysis of Large Scale Ocean Circulation, 1980. ISBN 9061961929.

MCT $121 \mathrm{~W}_{0} \mathrm{H}$. HAEMERS, Eigenvalue techniques in design and graph theory, 1980. ISBN 9061961947.

MCT 122 J.C.P. BUS, Numerical solution of systems of nonlinear equations, 1980. ISBN 9061961955.

MCT 123 I. YUHÁsz, Cardinal functions intopology - ten years later, 1980. ISBN 9061961963.

MCT 124 R.D. GILL, Censoring and Stochastic Integrals, 1980. ISBN 906196 1971 .

MCT 125 R. EISING, 2-D Systems, an algebaric approach, 1980. ISBN 906196 $198 \mathrm{X}$.

MCT 126 G. VAN DER HOEK, Reduction methods in nonlinear programming, 1980. ISBN 9061961998.

MCT 127 J.W. KLOP, Combinatory reduction systems, 1980. ISBN 9061962005.

MCT 128 A.A.J. TALMAN, Variable dimension fixed point algorithms and triangulations, 1980. ISBN 9061962013.

MCT 129 G. van der LAAN, Simplicial fixed point algorithms, 1980. ISBN 9061962021 .

MCT 130 P.J.W. TEN HAGEN a.o., ILP Intermediate language for pictures, 1980. ISBN 9061962048. 
MCT 131 R.J.R. BACK, Correctness preserving program refinements: Proof theory and applications, 1980. ISBN 9061962072.

MCT 132 H.M. Mulder, The interval function of a graph, 1980. ISBN 9061962080 .

AN ASTERISK BEFORE THE NUMBER MEANS "TO APPEAR" 
